\title{
REVIEW OF THE LITERATURE ON THE PRESCHOOL PEDESTRIAN
}

\author{
JOANNA PHINNEY \\ LAURA COLKER \\ MICHAEL COSGROVE
}

APPLIED MANAGEMENT SCIENCES, INC.

SILVER SPRING, MARYLAND

Contract No. DOT-HS-9-0-2264

October 1984

PREPARED FOR:

U.S. DEPARTMENT OF TRANSPORTATION

NATIONAL HIGHWAY TRAFFIC SAFETY ADMINISTRATION

WASHINGTON, D.C. 20590 


\title{
REVIEW OF THE LITERATURE ON THE PRESCHOOL PEDESTRIAN
}

\author{
JOANNA PHINNEY \\ LAURA COLKER \\ MICHAEL COSGROVE
}

APPLIED MANAGEMENT SCIENCES, INC.

SILVER SPRING, MARYLAND

Contract No. DOT-HS-9-0-2264

October 1984

\author{
PREPARED FOR: \\ U.S. DEPARTMENT OF TRANSPORTATION \\ NATIONAL HIGHWAY TRAFFIC SAFETY ADMINISTRATION \\ WASHINGTON, D.C. 20590
}


This document is disseminated under the sponsorship of the U.S. Department of Transportation in the interest of information exchange. The U.S.

Government assumes no liablitity for the contents or use thereof. 
Technical Report Documentation Page

\begin{tabular}{|c|c|}
\hline 2. Government Accession No. & 3. Recipient's Catalog No. \\
\hline 4. Title and Subtitle & $\begin{array}{l}\text { S. Report Date } \\
\text { October } 1984\end{array}$ \\
\hline \multirow{2}{*}{ Literature Review on The Praschool Pedestrian } & 6. Performing Organization Code \\
\hline & \multirow{2}{*}{$\begin{array}{l}\text { 8. Performing Organization Report No. } \\
\text { G- } 165\end{array}$} \\
\hline $\begin{array}{l}\text { 7. Authors) } \\
\text { Phinney, J., Coiker, L., Cosgrove, } M .\end{array}$ & \\
\hline 9. Performing Organization Name and Address & 10. Work Unit No. (TRAIS) \\
\hline \multirow[t]{2}{*}{$\begin{array}{l}\text { Applied Management Sciences, Inc. } \\
962 \text { Wayne Avenue, Suita } 700 \\
\text { Silver Spring, Maryland } 20910\end{array}$} & $\begin{array}{l}\text { 11. Contraet or Grant No. } \\
\text { DOT-HS- }-\mathrm{C}-\mathrm{C}-2264\end{array}$ \\
\hline & \multirow{2}{*}{$\begin{array}{l}\text { 13. Type of Report and Period Covered } \\
\text { Literature Review } \\
\text { November } 1979 \text { - August } 1981\end{array}$} \\
\hline \multirow{2}{*}{$\begin{array}{l}\text { 12. Sponsoring Agency Name ond Addess } \\
\text { J.S. Department of Transportation } \\
\text { Hational Highway Traffic Safety Administration } \\
4007 \text { th Street, S. H. } \\
\text { Hashington, D.C. } 20590\end{array}$} & \\
\hline & 14. Sponsoring Agency Code \\
\hline 15. Suppiementary Notes & \\
\hline
\end{tabular}

16. Abstroct

The purpose of this literature review was to describe (1) the factors leading to typical preschool pedestrian accidents, (2) the developmental characteristics of the jrescnool child that affect his/her behavior in traffic, (2) social factors that may place a preschooler at rist in traffic, (4) programs of traffic safety education for in-school and preschool use in the United states, and (s) preschool trafitc safety progranis developed by foreign countries.

Detailed information relevant to each of these objectives is presented in this volune.

17. Xeywords
Pedestrian, Praschool Traffic Safety
Education, Child Development, State
Tratio Sarety Programs, Foreign
Traffic Safe-y Prograns

18. Distribution Statement

This docunent is available to the U.S. public throuch the lational Technical Informetion Service, Springijeld, yiginia 22161

19. Security Classif. (of this report)

Unclassified
20. Security Clessif. (of this page)

inclassified
21. No. of Pages 22 . Price 465 


\section{ACKNOWLEDGEMENTS}

In the conduct of this literature review, we were ably and enthusiastically assisted by many people, whose efforts on our behalf are deeply appreciated. First of all, we wish to thank Dr. Marvin Levy, our contract technical manager at the NHTSA, for his assistance, encouragement, and support. We have also received guidance from Or. Alfred J. Farina, Jr., Mr. Lawrence Pavlinski, Or. Pame la T. Anikeeff, and Mr. Roger Kurrus, also of the NHTSA.

Our panel of consultants was instrumental in steering us to relevant literature, helping us locate obscure materials, and lending us personal copies of materials. We thank each of them: Mr. Don Lafond, Ms. Sylvia Ross, Mr. Richard Knoblauch, Prof. Carol Seefeldt, Ms. Charito Kruvant, Ms. Rita Weiss, and Mr. Jerry Sandler.

We have received invaluable assistance from another group of individuals whom we wish to thank: Ms. Peggy Matheson for translating the Norwegian materials, Mrs. Jean Bindenagel and Dr. George Kettner for translating the German materials, Ms. Kaija Wilson for translating the Finnish materials, Ms. Larilyn Reitnauer for translating the Swedish materials, Ms. Karen Johnson for her help in coordinating arrangements for translations, and Dr. Linda Cosgrove for sharing her expertise on vision with us.

We are also indebted to the Governor's Highway Safety Representatives and to the traffic safety officials both in this country and abroad who expedited our search for relevant materials. 
Lastly, we wish to gratefully acknowledge all of the researchers, curriculum developers, and authors cited in this volume whose previous efforts have laid the foundation for the development of The Walking in Traffic Safely (WITS) Program. 


\section{FOREWORD}

Over the past twenty years, accident data have consistently revealed that the young preschool child is overrepresented in traffic accidents. Some $16 \%$ of all pedestrian accidents in the United States involve children under six years of age (Reiss, 1978), even though this age group composes but $8 \%$ of the population. The extent of this problem is underscored by the fact that traffic accidents are the leading cause of death to the child under six (American Automobile Association, n.d.).

The situation that exists in the United States has been echoed worldwide. The Scandinavian countries have spent the last two decades trying to combat the problem. Norway, Sweden, Denmark, Finland, and Iceland have all developed ongoing campaigns to try to reduce the risks to the young child in traffic. Great Britain, both through its Tufty Club and through efforts sponsored by the Transportation and Road Research Laboratory (TRRL), has likewise strived to lower accident rates involving this target group of youngsters. West Germany, Austria, Canada, and Japan, among others, have also in recent years developed materials expressly aimed at reducing the number of traffic accidents involving preschool children.

Currently, the United States, under the auspices of the National Highway Traffic Safety Administration (NHTSA) of the Department of Transportation (DOT), is in the process of developing safety program materials aimed at reducing the dangers to the young child in traffic. Known as the Walking in Traffic Safely (WITS) Program for Preschoolers, this program is empirically rooted accident data analysis and child development theory. As the first step 
in the program, therefore, it was deemed necessary to examine the extant literature for guidance and direction. Specifically, it was decided to research these key issues:

(1) Who is most likely to be involved in a preschool pedestrian accident? Are there particular differences that distinguish those who are typically involved in pedestrian accidents from those who are not involved in accidents?

(2) Where do most preschool pedestrian traffic accidents occur? Is there any discernible pattern to these accidents?

(3) Do any particular predisposing factors place a young child at risk? Is there any relationship between a child's behavior and his/her likelihood of being involved in an accident?

(4) When do most pedestrian traffic accidents involving preschoolers occur? Are there specific times of day, days of the week, or seasons of the year which appear to be the most dangerous for youngsters?

(5) What are the developmental capabilities which impact on a preschooler's ability to cope effectively in traffic? How do developmental limitations place the young child at risk?

(6) What are the social/emotional needs of young children that might impact on their ability to handle traffic environments?

(7) What traffic safety education programs designed for preschoolers currently exist in the U.S.? What features of these programs should be incorporated into the WITS program?

(8) What traffic safety education programs designed for preschoolers currently exist abroad? What features of these programs should be replicated by the WITS program?

These research questions form the conceptual framework for this report. Questions 1 through 4 are addressed in Chapter 1, which presents a profile of the child pedestrian accident victim. Chapter 2 answers question 5 . In that chapter, a critical analysis of avallable child development literature is presented. Question 6 is the focus of Chapter 3. Chapter 4 addresses question 7. In the latter half of the chapter 4, a program-by-program analysis of all relevant domestic programs is presented. In Chapter 5 , question 8 is explored. As in Chapter 4 , the conclusion of this chapter is a program-by-program analys is of existing foreign programs. 
In compiling the information documented in this report, the procedures summarized below were followed. These procedures are listed in the chronological order in which they were implemented.

1. Appropriate resources at the NHTSA 1ibrary, The Library of Congress, The National Institute of Education Library, and the National Library of Medicine were identified and reviewed.

2. A computerized literature search of the ERIC, MEDLARS, Dissertation Abstracts, and Psychological Abstracts data bases was run. Identified resources were obtained and reviewed.

3. Foreign traffic safety experts were identified in consultation with the NHTSA and our panel of experts. Addresses for these individuals were obtained through a review of the literature and the assistance of the embassies of Finland, Norway, Sweden, Denmark, the Netherlands, West Germany, Great Britain, Japan, and Austria. Letters were sent to all identified experts requesting any available materials. Without exception, each of these individuals complied with our request. These documents were, in turn, reviewed by staff. A listing of the names and addresses of those experts who so kindly supplied us with these foreign publications is included as Appendix C to this document.

4. The Governor's Highway Safety Representatives in each state and U.S. territory were contacted by telephone or letter. As with the foreign contacts, requests were made to these individuals for appropriate materials. In response to our request, the representatives not only provided us with materials, but in many cases, suggested additional persons to contact in our search. All such contacts were made, and additional materials and research reports were obtained for review.

5. A number of private organizations in the U.S. were broached in regard to the traffic safety programs they sponsor and/or have developed. These organizations included The American Automobile Association. The Automotive Safety Foundation, Film Loops, Inc., Highway Users Federation for Safety and Mobility, National Education Association, National Safety Counc il, National Safety Town Center, and the Sears Roebuck Foundation. Many of these organizations graciously provided us with complimentary copies of their materials for review.

6. A computerized 1 iterature search of preschool and elementary school pedestrian programs was run for us by the Highway Safety Research Institute of Ann Arbor, Michigan. Selected materials from this listing were purchased from the Institute's library for review.

7. Overseas organizations that developed/produced/distributed traffic safety materials for use in foreign countries were identified for us by foreign experts (as part of procedure 3 ). With the assistance of the embassies of the countries involved, these organizations were 
contacted with letters of request for materials. Without exception, these organization provided us with complimentary copies of their materials. In many instances, the materials included records, films, and games in addition to printed matter. In order to review these materials, the services of six translators were obtained.

Unfortunately, fiscal constraints prohibited the translation of all received material into English. Only those materials which (a) had been singled out in the literature as exemplary and (b) did not entail extensive translation fees were able to be translated. translated materials were reviewed by staff.

The sum product of the seven steps outlined above is this document. By answering our research questions, the information analyzed in this report has served as a blueprint for the development of NHTSA's WITS program for preschoolers.

In reading this document, the reader is advised that materials reviewed in this report include only those available as of August 1980 . Pertinent materials published subsequent to this time are not referenced, as this report represented a preliminary working document. The Literature Review is presented intact in the form in which it was originally completed; the only changes to this report have been to update publication information for those materials which were "in press" during the time that the materials were originally reviewed but have subsequently been published.

In addition, in reviewing the domestic and foreign programs highlighted in Chapters 4 and 5 , the reader is encouraged to consuit an evaluation tool recently developed under contract to the NHTSA, the Program Assessment Kit for Pedestrian and Bicyclist Safety Education Programs. Through use of this instrument, the reader can determine the safety relevance (i.e. the extent to which a program contains information consistent with the findings of NHTSA-sponsored research), instructional appropriateness, and suitability of the program materials examined in Chapters 4 and 5.

As implied, it is our intention that the material presented in this report be useful in contexts that transcend our one project. The effort that went into the preparation of this document will have been well spent if planners, educators, and parents alike can use this information to promote the well-being of all young children in traffic. 
TABLE OF CONTENTS

PROFILE OF THE CHILD ACCIDENT VICTIM. . . . . . . . . . 1

I. Who is Likely to be a Potential Victim? . . . . . 1

I.A. Sex: A Survey of the Literature. . . . . . . 1

I.B. Personality: Factors Which Increase Children's Probability of Involvement in Traffic Accidents............

I.C. Parental Supervision: A Survey of the Literature................. 10

I.D. Birth Order: A Survey of the Literature. . . 18

II. Where is the Accident Likely to Take Place? . . . . 20

III. What Does the Potential Victim do that Puts

Him/Her at Risk?................

IV. When is the Child Accident Victim Most Likely

to be at Risk?............... 26

IV.A. Time of Day and Accident Causation. . . . . 26

IV.B. Day of the Week and Accident Causation. . . 28

v. Summary .................. 33

2 DEVELOPMENTAL CAPABILITIES OF YOUNG CHILDREN. . . . . . 35

I. Developmental Profile ............ 35

I.A. Egocentrism .............. . 37

I.B. Transductive Reasoning.......... 37

I.C. Centering. . . . . . . . . . . . 38

I.0. Inability to Conserve. . ......... 39

I.E. Distortion of Reality. . . . . . . . . . 40

II. Traffic Related Capabllities............ 41

II.A. Vision................ 41

II.B. Audition. ............... 49

II.C. Attention ............. 53

II:D. Spatial Relations........... . 58

II.E. Distance Perception ............ 60

II.F. Perception of Velocity. . . . . . . . . 63

II.G. Causality.............. 66

II.H. Short-Term Memory. . . . . . . . . . . 67

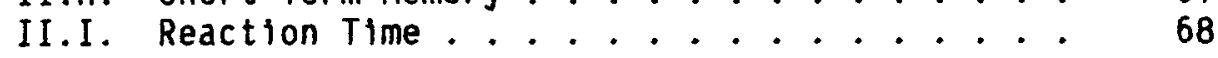


TABLE OF CONTENTS (Continued)

II.J. Motor Behavior. ............ 70

II.K. Impulsivity................ 72

II.L. Risk Perception............ 73

III. Educational Capabtlities of Preschoolers...... 74

III.A. Long-Term Memory ............. 74

III.8. Understanding Rules. . . ........ 76

III.C. Concept of Left and Right. . . . . . . . 78

I[I.0. Communication. ........... 78

III.E. Vocabulary ................ 81

III.f. Traffic Signs and signais. . . . . . . . . 83

III.G. Logical Operation. . . . . . . . . . . 83

III.H. Crossing Judgment. . . . . . . . . . 85

III.I. Route Planning............. 86

IV. Summary. . . . . . . . . . . . . . 87

3 SOCIAL AND PSYCHOLOGICAL CHARACTERISTICS OF

PRESCHOOL CHILDREN. . . . . . . . . . . . . . . . 89

I. Soctal Growth During the Preschool Years...... 89

II. The Role of Play in the Preschooler's Development. . 92

III. Emotional Development in the Preschooler...... 94

IV. The Development of Imagination .......... 98

V. Irrational Fears in Preschool Children ....... 99

VI. Parents as Models................. 91

VII. The Development of Trust............ 112

VIII. The Development of Curiosity........... 103

IX. Conclustons. . . . . . . . . . . . 105

4 CURRENT PEDESTRIAN SAFETY PROGRAMS FOR PRESCHOOL CHILDREN 106

I. A History of Pedestrian Safety Education . . . . . 106

I.A. The Growth of Safety Education....... 108

I.B. A Lapse in Safety Education. .......... 109

I.C. Federal Impetus for Traffic Safety Education. 111 
TABLE OF CONTENTS (Continued)

Chapter

Page

I.D. Important Contributions to Traffic

Safety Education............ . 112

I.E. Advent of Preschool Pedestrian Safety $1 . . . .114$

I.F. Conclusion............... . . 117

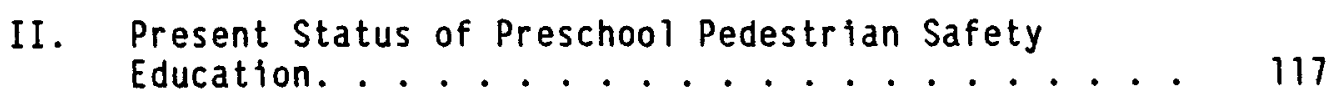

III. Types of Preschool Pedestrian Programs . . . . . . 118

III.A. The State Curriculum Manual Approach . . . 118

III.B. The Coloring Book Approach ... . . . . 124

III.C. The Safety Town Approach . . . . . . . . . 125

III.D. The Officer Friendly Program Approach. . . . 126

III.E. Children's Traffic Club Approach ...... 127

III.F. The Parent Child Book Series Approach. . . 128

III.G. The Media Approach ............. 129

IV. Pedestrian Concepts Taught to Preschool Children . . 130

IV.A. What Should be Taught in Preschool Pedestrian Safety Education? ....... 130

IV.B. Which Concepts Can Preschool chilidren Under stand? . . . . . . . . . . . . 133

IV.C. Which Concepts Have Safety Relevance?... . 135

IV.D. Are Concepts Taught in Sequential Order? . . 136

V. Pedestrian Instruction Given to Preschool Children . 137

V.A. How Are Pedestrian Instructions Used? ... 137

V.B. Which Pedestrian Instructions are Taught? . . 138

V.C. Do Pedestrian Instructions Vary Across
Programs? . . . . . . . . . 138

V.D. How Explicit Should Pedestrian
Instructions Be?......... 139

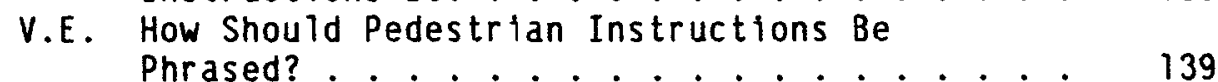

V.F. Should Pedestrian Instructions Consist of Do's and Don'ts? .......... . 139

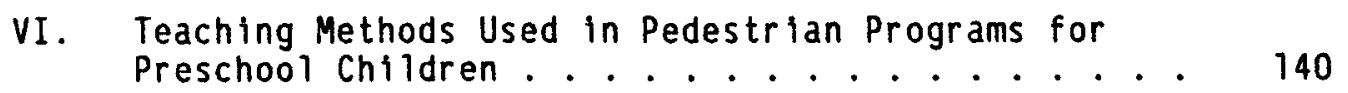

VI.A. Which Teaching Methods Are Used? ... . . . 140

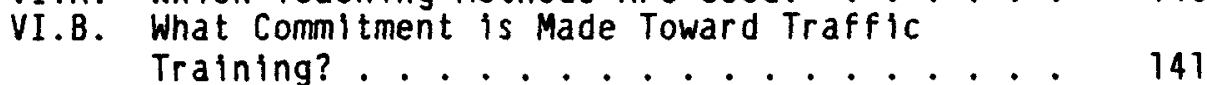


TABLE OF CONTENTS (Continued)

Chapter

Page

VI.C. Is There a Place for Active Learning and Discovery in Pedestrian Education? ..... 145

VI.D. What Types of Materials are Designed for Children?............... 146

VI.E. Which Teaching Methods are Appropriate for Preschool Children?.......... 148

VI.F. Teaching Knowledge. ............ 150

VI.G. Teaching Approprlate Behavior:....... 155

VI.H. Detalled Descriptions of Pedestrian Safety Programs........... . 158

5 PEDESTRIAN PROGRAMS FOR PRESCHOOL CHILOREN OUTSIDE THE UNITED STATES . . . . . . . . . . . . . 356

I. Types of Preschool Pedestrian Program Materials. . 358

I.A. School Approach. ............. 358

I.B. Television Series Approach ... . . . . . 359

I.C. Print Materials Approach ........... 359

I.0. The Children's Traffic Club Approach..... 360

II. Concepts Taught to Preschool Children........ 362

III. Pedestrian Instructions. ............. 362

IV. Teaching Methods.......... . . . . . . 364

V. Information Given to Parents.......... 365

VI. Summary. . . . . . . . . . . . . . 366

v. Detalled Descriptions of Pedestrian Safety
Programs Outside the United States......... 367

APPENDIX A: REFERENCES

APPENDIX B: LITERATURE REVIEW METHODOLOGY

APPENOIX C: TRAFFIC SAFETY CONTACTS IN FOREIGN COUNTRIES 


\section{LIST OF EXHIBITS}

Exhibit Page

4.1 Current Status of Preschool Safety Education ...... 119

4.2 Tally of Concepts Taught in Preschool Pedestrian

Programs......................... 131

4.3 Prevalent Teaching Methods Used in Pedestrian Programs

for Preschool Children.............. 142

4.4 Learning Experiences Teaching Methods Provide...... 151 


\section{1 \\ PROF ILE OF THE CHILD ACCIDENT VICTIM}

In attempting to develop The WITS materials, an important first step was the identification of the preschool pedestrian accident victim. By identifying the attributes of potential victims, the times and places when they are most heavily at risk, and the social and demographic characteristics which seem to influence their behavior in traffic, countermeasures could be developed that were aimed at reducing the risks lo these youngsters. With this goal in mind, Lhis section of the Literature Review presents a general profile of the potential preschool accident victim.

I. WHO IS LIKELY TO BE A POTENTIAL VICTIM?

\section{I.A. Sex: A Survey of the Literature}

In describing the potential victim of a traffic accident, one fact stands out in all of cross-cultural research: more boys than girls are involved in traffic accidents. Without exception, a country-by-country analys is of accident reports upholds this finding. In the Untted States, three major studies sponsored by the DOT have reported that preschool boys are more frequentiy involved in traffic accidents than are preschool girls. First of a11, in the sludy of pedestrian Safety: The Identification of Precipitating Factors and Countermeasures, known hereafter as the ORI study, ${ }^{\text {/' }}$ (Snyder \& Knoblauch, 1971) it was found that $71.2 \%$ of all trafic accident victims aged

1/Contact No. FH-11-7312 
under four were male. In fact, the preschool age category showed the largest proportion of males involved in accidents for all of the age groupings recorded by this 1969-1970 study of 13 U.S. cities.

In a second study sponsored by NHTSA-FHWA during 1972-1974, the Study of Urban Pedestrian Accident Countermeasures Experimental Evaluation, descriptive data on 2,044 pedestrian accidents in the urban areas of six major cities were collected and analyzed. This study, specified hereafter as the Urban study, confirmed the ORI finding: $68 \%$ of all pedestrian accident victims in the under four age range were male (Knoblauch, 1975).

Other more recent governmental studies have also underscored the predominance of boys in traffic accidents. The NHTSA-sponsored study, Causative Factors and Countermeasures for Rural and Suburban Pedestrian Accidents, $2 /$ in examining some 1,531 pedestrian accidents in rural sites within six states from 1973-1976, found an even higher percentage of males in the accident statistics (Knoblauch, 1977). According to this study, which is referred to here as RUPED, $74 \%$ of the accident victims under four were male. Another 1977 study of some 4,386 pedestrian and bicyclist injuries in 32 jurisdictions likewise found that $62 \%$ of accident victims were male (Wolfe, 19791 .

State and local analyses of traffic accidents have echoed these national findings. In Detroit, for example, it was found in a 1963 analysis, that twice as many boys as girls were involved in traffic accidents (Logan, 1963). The American Automobile Association (1965) summarizes the situation in this manner: "Regardless of age, boys are involved in seven out of every ten child accidents" (p. 5).

Accident data in other countries present a similar picture. Lindensjo, Mattson, and Sandels (1971), writing in the Skandia Report, describe an analysis of 1,906 Swedish traffic accidents involving children ten and under

$1 /$ Contact No. DOT-HS-190-2-480

$\underline{2}$ Contact No. DOT-HS-355-3-718 
during the years 1968-69. In that study, conducted jointly by the Skandia Insurance Company and the Institute for Child Development Research, it was noted that, "Ouring the years in question, 1968-69, approximately twice as many boys as girls were involved in traffic accidents" (p. 9). In terms of pedestrian accidents only, there were 435 female accident victims as compared to 769 male accident victims. In an earlier study reported in Sweden, Magnusson (1966) reviewed all articles on traffic accidents that occurred to children under age 15 that were reported in the Index Medicus. Magnusson found that "There is a strong domination for boys in the statistics of traffic accidents to children. The difference between boys and girls is hardly recognizable during infancy, but increases continually with age. A summary of sex differences from a couple of reports gives the following result: of traffic-injured children at the age of one to four years, $62 \%$ are boys and $38 \%$ are girls ..." (p. 257).

In Japan, it is also reported that, "At any age, more victims are found among boys than girls ... four out of ten boys sustain injuries by accident while only two out of ten for girls." (0kamoto, 1978, p. 122).

A study in Montreal, Canada, in 1959-1960, produced similar results (McDouga 11, 1960). The author of the study concluded that boys were more often killed than girls were when they were pedestrians but not when they were passengers in a car. Another study conducted in Vancouver (Read, Bradley, Morison, Lewall, \& Clarke, 1963) of 713 traffic accidents involving children aged one to fourteen, found that girls as compared to boys were injured in a 1:7 ratio. The authors concluded that, "In the first 7-8 years of 1ife, more boys than girls are hit by motor vehicles" (p. 10).

In New South Wales, it has been reported that twice as many boys are involved in pedestrian accidents as girls (Avery, 1974). In Great Britain, Grayson's (1975a) year-long study of 411 youngsters injured in traffic accidents in Hampshire also revealed this same 2:1 ratio. Grayson writes, "Nearly twice as many boys as girls were involved in an accident while out playing ..." (p. 2). Preston (1972) likewise confirms that trend in England. In analyzing accident data concerning 980 children injured in Manchester and Salford in 1969, she writes, "At all ages from 2-11 years there are more boys than girls injured" (p. 32). 
A final summary of this topic was presented by Jarvis (1978) at a Joint Australian Road Research Board (ARRB)/Australian Department of Transportation (DOT) Pedestrian Conference: "Overall and for most individual age groups, the male fatality rate is double the female rate. For injuries, the male rate is approximately $1-1 / 4$ to $1-3 / 4$ the female rate" (p. 6$)$.

\section{A.1. Sex: Possible Explanations Why More Boys Than Girls Are Injured in}

\section{Pedestrian Traffic Accidents}

That more boys than girls are both injured and ktlled in traffic accidents is a documented fact. In no country surveyed in the literature did the ratio of boys to girls even approach its occurrence in the population base rate. Two theories have been espoused as possible explanations as to the reported difference. One theory is related to exposure to traffic dangers, the other to behavior in traffic.

\section{Exposure}

The "exposure" explanation for the overrepresentation of boys in the preschool accident data attributes this fact to the bellef that boys spend more time as pedestrians than girls and are, consequently, more exposed to danger. Sande 1s, writing in 1974, comments on the fact that in Sweden there is an exposure differential: "Parents are more likely to allow young male children to play near traffic than young females" (p. 15 ).

Other European studies have tended to confirm this play differential. In a study conducted for the British Transportation and Road Research Laboratory (TRRL) in 1972, Sadler interviewed 2,017 mothers of two to eight year old children to find out information on these three items: (1) the exposure risk to the child; (2) the amount of training the child has been given in road safety; and, (3) background information on the child's family. To gather information on the child's risk in traffic, Sadler asked mothers where their children played. She found that "At all ages, giris are less. likely than boys to play in the street" (p. 15). 
While some researchers have taken this conclusion as evidence of a link between exposure and accident involvement, other researchers refute this claim. Routledge, Repetto-Wright, and Howarth (1974) vigorously deny that any such relationship can be drawn using Sadler's data. They write:

\begin{abstract}
The measure of exposure in relation to the accident statistics provides an estimate of the risk involved in allowing a child of a given age and sex out of the house, and nothing else. It is not possible to state that one group of children behaves more dangerously than another, since any relationship found between being in the street and number of accidents could arise for several reasons (p. 460 ).
\end{abstract}

In an attempt to bridge the gap between exposure per se and its relationship to accidents, Routledge et a1. conducted a study in 1970-1971 of 288 five to eleven-year-old school children in Nottingham City. Using a framework in which measures of exposure were defined and related to the accident categories of pedestrians in different traffic situations, the authors found no sex differences in exposure below age eight. The authors conc luded:

The results of ...studies of children's exposure ... show little evidence of a difference in the exposure of boys and girls, particularly in the younger children, where the difference in accident rates is so marked. They do show a pronounced and statistically highly significant increase in exposure with age for both sexes, whereas the accident rates, especially for boys, are declining steeply over the age range studies (pp. 478-479).

The results of the exposure studies as surveyed above do not support a relationship between exposure and being involved in traffic accidents. Although there is some indication, especially in European studies, that preschool boys are allowed to play in the roadway more of ten than girls and thus may be more exposed to danger, it does not seem that this exposure can be tied to accident data. The one study which attempted to examine this relationship (Routledge et al., 1974) found no evidence of this occurrence. Although the empirical data on this subject are limited, exposure, in and of itself, does not seem to be able to account for the overrepresentation of males in the preschool accident data. 
Behavior

While exposure in and of itself does not appear to be directly linked with accident causation, there does appear to be some evidence that behavioral differences between the sexes can be tied to accident involvement Routledge et a1. (1974) reason, "we could conclude that the reasons for the differences in the accident rates between ...groups are not to be found in the children's exposure to traffic but must be looked for in their behavior when crossing roads," (p. 479). Cross-cultural research has consistently shown that the "typical" preschool boy may be more susceptible to accidents because of his behavior than his female playmates. In a previously referenced study, Read, et al., (1963) concluded that "the aggressive behavtor of young boys makes them likely accident victims." While the authors do not define "aggressive," they juxtapose the boys behavior with the "cautious" behavior of their female agemates.

Sandels (1975) likewise points to the fact that in many Swedish studies conducted prior to the implementation of Sweden's Children's Traffic Club boys consisiently took more risks in traffic than did girls. A study conducted by Sandels in May of 1961 in two districts of Stockholm with 85 children aged four to seven, led the author to conclude that "Boys were poorer pedestrians than girls, both when the children were on their own or together with peers" (p. 55).

In Britain, Routledge et a1. (1974) observed children's "risk" behaviors in traffic settings. Risk was assessed by the authors by relating exposure measures to accident statistics. The results of this study showed that after allowance had been made for exposure, the younger boys below age $B$ were taking approximately twice as much risk in crossing the road as girls of the same age" (p. 467).

In a Japanese study of nurseries in Takamatsu City, Tanaka (1965) attributed the sex differential to the fact that boys are more active than 
girls. - In New South Wales, Avery (1974) likewise notes that Australian boys are encouraged to exhibit many of the behaviors which are associated with risk-taking in traffic. Research by Husband (1973) confirms this pattern of aggressive, risk-taking behavior in young boys.

It should be noted, though, that in two studies surveyed in the literature, sex differences in children's behavior in traffic were not reported. In a speech given in 1966 at the Second Congress of the International Association for Accident and Traffic Medicine, Sandels reported these results from a survey of preschool children's activities performed in Sweden from 1958-1964:

Starting with an investigation in a couple of suburbs of Stockholm we found that from the age of 2 and through the preschool years (compulsary school age in sweden is 7 ) successively more children were found in all type of open-air places-with reference to traffic, dangerous as well as secure. From age 3 on, the amount of children in dangerous places increased, and among the 5-and-6 year olds we found nearly as many children in dangerous places as safe ones... There were almost as many girls as boys in the dangerous places (p. 230).

A second more recent paper by Russam (1977) reports on Grayson's (1975b) study of pedestrian crossing behavior in Reading, Windsor, Blackwell, and Camberley. In reviewing this study, Russam writes:

When the road crossings of the children were analyzed in detail, sex differences in behavior were only infrequently found at some of the sites... The lack of detected behavioral differences was again contrary to what might have been expected from the data.

In examining the above two reports, care should be taken not to generalize these finding beyond the context in which they were made. As already noted, in more recent studies, Sandels has documented sex differences among the

1/Tanaka goes on, however, to further attribute the differential to the fact that "girls apparently understand traffic rules better than boys," (p. 123). While this conclusion may be due to cultural differences in instructional techniques, it is not one which is substantiated by the ijterature. The next chapter of this report will address this issue more fully. 
behavior of preschoolers in traffic. In Children in Traffic (1975), for example, she writes, "The comparison between the behavior of girls and boys when they were out alone or together with peers showed very considerable differences ..." (p. 53).

Likewise, in examining Russam's comments, it should be noted that while his observations are reflective of Grayson's conclusions, there were nonetheless some sex differences observed among the younger children (1.e., those five to eight years 01d). To this point Grayson (1975) writes, "At both the primay school sites boys were more likely to run across the road than were girls, but there were not sex differences in the older age groups" ( $p .7)$. Morever, in a second study of child pedestrian behaviors which inciuded younger preschoolers in the sample, Grayson (1975a) detected significant differences in the traffic behavior of boys and girls. He writes, "The results support the traditional belief that boys are more likely to run across the road than girls, but this difference was only found with the two younger age groups (1.e., those aged 0-4 and 5-9 years)" (p. 8).

Consequently, it would seem that the preponderance of evidence in the European studies supports the premise that young boys exhibit riskier behavior in traffic than do girls.

In the U.S., studies have substantiated this assumption. The DOT-sponsored study of Pedestrian Safety: The Identification of Precipitating Factors and Countermeasures (Snyder \& Knoblauch, 1971) found that male preschoolers were more easily distracted in traffic situations than were female preschoolers. NHTSA's study of Safety Messages for Public Education Programs (Blomberg \& Preusser, 1975) confirms this pattern: "... The play patterns of young males and females differ, with males more likely to engage in sports which could lead to distraction from proper pedestrian behaviors" (p. 26 ).

Another NHTSA research study (Thackray \& Dueker, 1983) observed 2,213 children aged one to eleven in a variety of street-side and in-the-street situations in five cities. The results of this study revealed that males were involved in over two-thirds of the behaviors designated as "high risk." High 
risk behaviors included those play related activities such as throwing or catching a ball that involved street entry without prior looking for oncoming cars.

In sum, U.S. research confirms the existence of the same sex differences in the behavior of young children in traffic that has been reported in European research. More than exposure, it appears that the overrepresentation of preschool boys in traffic accidents can be attributed to their roadside behavior.

\section{I.B Personality: Factors Which Increase Children's Probability of}

\section{Involvement in Traffic Accidents}

In trying to determine why it is that proportionally so many boys are injured in traffic, many researchers have tried to establish whether personality factors may predispose the male child to danger. Several international authors have explored this subject.

In their study of traffic accidents in Vancouver, Read et a1. (1963) found that the accident victims were predominantly described as "impetuous" and "venturesome." The authors write:

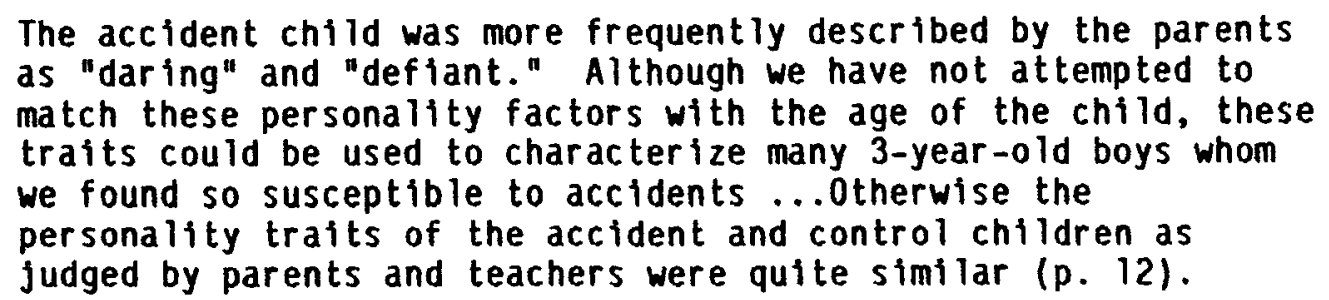

In New South Wales, Avery (1974) has commented that the stereotypical behavior of the young Australian boy is "aggressive," i.e., "determined, daring, and fearless." Those traits, he infers, make the child particularly susceptible to accidents.

Ekstrom, Gastrin and Quist (1966) in Sweden also attempted to study the relationship between personality and injury in traffic. By comparing 706 child accident victims with 88 "soclal twins," the researchers set out to see 
how the accident victims differed in personality from the control group. By inquiring of parents whether the child was "lively," "nervous, "or "quiet," the authors hoped to establish a personality pattern. These terms were not further defined, so it was left to the respondents to supply their own interpretations of the descriptors. The authors hypothesized that the accident victims would more of ten be lively than quiet. While this trend did appear, it was not markedly so. Moreover, the differences between the accident group and control group were not clear cut, perhaps because parents in both groups preferred to think of their children as "lively" rather than "quiet" or "nervous."

From the few studies available in the literature it does appear that there is some relationship between personality and susceptibility to involvement in traffic accidents. While the Ekstrom et al. (1966) study produced no behavioral/personality differences, the categories used to describe the children, as noted, were rather vague and not sharp discriminators of personality. On the other hand, Read et a1. (1963) and Avery (1974) noted a behavioral/personality factor which predisposes children to traffic accidents. The aggressive, outgoing, defiant behavior which stereotypically characterizes three-year-old male toddlers seems, to those authors, to be the type of behavior which places a chlld at risk in traffic.

\section{I.C. Parental Supervision: A Survey of the Literature}

One factor examined in the literature which consistently influences a child's traffic behaviors, is parental supervision. Studies both in this country and abroad have noted that the lack of parental supervision contributes to children's traffic accidents.

The degree of parental supervision has been examined on several levels: (1) whether the parent permits the child to play in and/cross the road by himself/herself; (2) whether the parent allows the child to play in and/or cross the road in the accompaniment of a peer: (3) whether the parent allows the child to play in and/cross the road only with another older child being present; and (4) whether the parent allows the child to play in and/cross the road only in the presence of an adult. Studies have dealt with various facets of this continuum of parental supervision. 
In Britain, several researchers associated with the Transportation and Road Research Laboratory (TRRL) have examined the relationship between parental supervision and traffic safety. Sadler (1972) in her interview of 2,017 mothers of two to eight years olds in England, Scotland, and Wales found that a surprising number of parents permitted their young preschool child to play in the street. Sadler found that accessibility to a yard or a safe play area did not necessarily diminish street playing. She states, "Although possession of a yard makes it less likely that a child will play in the street, almost half of the 2-4 year olds who have access to a garden play in the street occasionally at least, and for 5-8 years olds this proportion rises to two-thirds" (p. 15).

In terms of road-crossing behavior, Sadler found that even among two-year-olds, over one-fourth were permitted to cross the street on which they lived by themselves. With each succeeding age group, the increase in children allowed to cross the road was dramatic. The following table records the breakdowns by age which Sadler found (p. 76):

\begin{tabular}{|c|c|c|c|c|c|}
\hline \multirow{2}{*}{$\begin{array}{l}\text { Population of children in } \\
\text { each age group who }\end{array}$} & \multicolumn{5}{|c|}{ Age of child } \\
\hline & 2 & 3 & 4 & 5 & 6 \\
\hline \multirow[t]{2}{*}{ Are allowed to cross streets } & \multicolumn{5}{|c|}{ Percent } \\
\hline & 26 & 48 & 64 & 77 & 86 \\
\hline \multirow[t]{2}{*}{$\begin{array}{l}\text { Are prohibited from crossing } \\
\text { streets }\end{array}$} & \multicolumn{5}{|c|}{ Percent } \\
\hline & 74 & 52 & 36 & 23 & 14 \\
\hline
\end{tabular}


Sadler's findings are even more dramatic in light of the fact that the amount of traffic on the road does not appear to markedly deter parents from keeping their children out of the road. Sadler writes: "Even where their mothers considered the road to be very busy, 13 percent of 2 year olds were able to cross it on their own" (p. 79).

The evidence of Sadler's survey is that even the very youngest preschoolers are permitted in the street by themselves. As has been noted in this report, because her data were retrieved independently of an analysis of traffic accident reports, the relationship between parental reports and the probability of being involved in a traffic accident is unknown. On the other hand, the fact that so many mothers admit to allowing their children in the street at an age before they are capable of coping effectively in traffic (See Chapter 2), does lead one to infer that the absence of parental supervision puts these children at risk.

Grayson, another British researcher, has also explored the topic of parental supervision in two studies conducted for the TRRL. In his observations of 1,790 pedestrians made at four sites, Grayson (1975b) noted how children crossed the road when alone, with a peer, and with an adult. While he, like Sadler, did not attempt to relate behavior to accident data, he did note which situations placed the child at greater risk. Interestingly, Grayson found that children demonstrated the least safe crossing behaviors when in the company of adults. He writes:

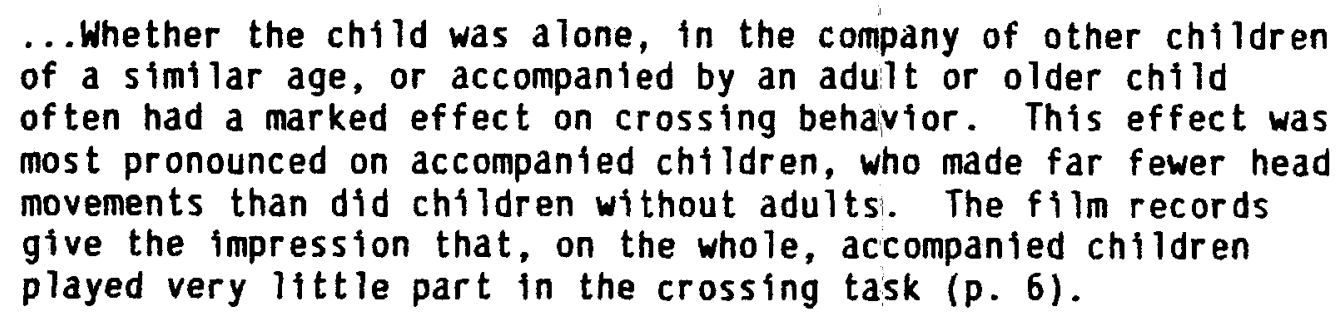

Grayson's observation underscores an important point. Supervision in and of itself does not ensure traffic safety. If the quality of the supervision is not high, then the child may face even greater risks than if he/she were unaccompanied. Sandels (1975) has made this observation from swedish studies as we11. She notes that young children are often least careful in crossing a 
street when they are in the company of an adult. It is Sande $15^{\prime}$ interpretation that children abdicate all responsibility for their well-being to adults whom they trust to get them safely across the street.

Grayson (1975a) examined the supervision/injury relationship in a second study performed at Hampshire during 1972. This study, unlike the earlier ones noted, attempted to relate behavior to accident data. For children four and under, Grayson found that nearly one-third were alone at the time of injury. Only a slightly larger percentage of children were in the accompaniment of an adult. The percentage of children in each category of accompaniment was observed as follows (p. 5):

\begin{tabular}{|l|cc|}
\hline Accompaniment pattern: & $\begin{array}{c}\text { Children } \\
\text { aged } 0-4\end{array}$ & $\begin{array}{c}\text { All children aged } \\
0-14\end{array}$ \\
\hline Child a lone & $\begin{array}{c}\% \\
42\end{array}$ \\
\hline Child with adult & 38 & 12 \\
\hline Child with older children & 22 & 20 \\
\hline Child with other children & 8 & 24 \\
\hline $\begin{array}{l}\text { Total } \\
\text { Base }\end{array}$ & $\begin{array}{c}100 \\
85\end{array}$ & $\begin{array}{l}100 \\
464\end{array}$ \\
\hline
\end{tabular}

As with his other study, Grayson found the quality of the supervision provided by parents of accident victims to be sorely lacking. He observed: "The finding that over a third of the pre-school accident victims were in the charge of an adult suggests that the supervision could have been substantially improved" (p. 12).

Preston's (1972) analysis of accidents in Manchester and Salford also revealed a lack of supervision. Here, it was not so much a question of poor 
supervision, but of missing supervision entirely. For children five and under in her study, she observed that two-thirds were injured within 100 yards of their home. In $92 \%$ of these cases, the children were unaccompanied at the time of the accident. In the other one-third of accident cases, over half of the children were in the company of an adult. Here, too, poor supervision seems to have been a key factor.

In Canada, similar findings have been found. In comparing the supervisory behavior of parents of accident victims in Vancouver with parents of a control group, Read et al. (1963) found that parents of the accident children left their children unsupervised in traffic at a considerably earlier age than did control parents. The authors describe their findings as follows:

The "accident parents," on the whole, discontinue supervision of the child's play area at an earlier age ( 4 years) than the control families ( 6 years). This difference appears in spite of the strong feeling on the part of the interviewers that the accident families were very guarded in their reply to these questions.

It was somewhat disconcerting, if not entirely unexpected, to find that the child's playground was usually the sidewalk and street. This held true even though the child might be of pre-school age and had a yard in which to play. The community playground was occasionally used if it was within five blocks of the child's home, but it was almost never his usual play area. Although not specifically compared, one would expect this to be particularly true for the younger children who would meet with many hazards in travelling to and from the playground unless they were under supervision ( $p, 13)$.

In Sweden, where several studies have explored this topic, a similar picture emerges. A study by Mattson and Lindensjo for the Skandia Insurance Company in Sweden during 1968-1969 (Sandels, 1974) revealed that except for toddlers aged one and two, accident victims were most of ten alone or with peers at the time of their accident. A breakdown of their data (Sandels, $1974)$ reveals the following profile (p. 8): 
Number of children alone, with friends, with adults

\begin{tabular}{|ccccc|}
\hline Age & Child alone & Child with friends & Child with adult & Total \\
\hline 1 & 1 & - & 2 & 3 \\
\hline 2 & 4 & 5 & 10 & 19 \\
\hline 3 & 8 & 6 & 6 & 20 \\
\hline 4 & 10 & 7 & 3 & 20 \\
\hline 5 & 11 & 4 & 5 & 20 \\
\hline 10 & 8 & 2 & 20 \\
\hline
\end{tabular}

A further interpretation of these data for Skandia by Sande 15 (1974), the so-called Skandia II Report, echoed the earlier findings. Sandels writes:

Even one-year-olds have been left out of the sight in the dangerous vicinity of parked cars, even if it had been only for a moment. Streets are crossed without holding the one-year-olds by the hand or on reins. A one-year-old has been lef $t$ alone for a time long enough to permit him to walk alone up to a highway, where cars were passing by very fast. Almost half of the two-year-olds are allowed to play outside alone or with friends in dangerous surroundings, which also is the case with more than half of the three-year-olds and all of the following age groups (p. 26$)$.

In 1970, Sandels reported on further studies that had been conducted by the Research Institute of Child Psychology at the University of Stockholm from 1960-1968. In one study conducted in Stockholm in 1960 of 550 children, Sande 75 reports that $80 \%$ of the children observed were alone or in the company of their peers while in traffic. The author concludes the following:

Children as young as 3 and 4 years were seen in more than one quarter of the cases in places which were dangerous from the traffic point of view, such as roadways, pavements and parking 
areas. They mostiy played alone while their mothers tried to keep them under observation from windows or balconies. Five and 6-year-olds had less supervision out-of-doors, and nearly half of them had chosen unsuitable playing areas ... Interviews with mothers confirmed that children as young as $21 / 2-3$ years of age were let out alone, and that mothers living in high-rise flats had difficulty in supervising their children (p. 112).

Sande is (1974) sums up the results of the many Swedish studies in this way: "Those who at the time of the accident were responsible for supervising the children let them out alone much too early" (p. 19).

In Australia, Cameron, Stanton, and Milne (1976) likewise found that children in accidents were most likely to have been unsupervised at the time of their accidents. The authors developed a risk calculation that reflected the risk to a pedestrian when crossing the street. For young pedestrians who crossed the street alone a risk factor of 25.0 was assigned. The presence of another walker drastically reduced the risk factor to 0.59 . No indication was given in the study, however, for risk factors that related specifically to an accompanying adult, older child, or peer. The conclusion from this study was most decidedly in the favor of supervision per se. In The Australia study, the quality of the supervision does not seem to be as important as does the presence of supervision in and of itself.

This finding was postulated earlier by Magnusson (1966) in an eight-year survey of child pedestrian accident victims reported in the Index Medicus. In his study, Magnusson found that three-quarters of the accident victims were either alone or in the company of another child during the time of their accidents. For our purposes, though, it should be noted that this figure is somewhat inflated since Magnusson examined cases of children aged one to fourteen, and older children would developmentally be expected to be on their own in traffic more than younger children.

Here in the U.S., a research study for NHTSA examined the very issue of parental supervision as it relates to the young child in traffic. As part of that effort (Thackray \& Dueker, 1983), children aged one to five in five cities were observed. Two major findings of this study were as follows: 
- Children observed playing in a near-the-street setting were without adult supervision more than $80 \%$ of the time they were observed.

When supervision was present, it appeared to suppress behaviors that could lead to an accident (e.g., dart-out type street entries).

This study, because it was not keyed to accident data, is similar to the work done by Sadler and Grayson. Moreover, like Sadler, Sandels, and Read et al., the American study noted a tremendous lack of supervision of young children in traffic. Even the very youngest of children were left to fend for themselves in traffic.

From the above survey of the literature, it is clear that supervision can play a major role in protecting the child in traffic. Why then are parents and guardians so neglectful of their children?

One explanation is that parents simply overestimate the capabilities of their young children in traffic. Sandels (1974) puts it this way:

In spite of all that has been written and said during the past few years about children's limited ability to manage in traffic, it is apparent that the supervisors too of ten lack knowledge about what can be expected of small children... There is no other way of explaining their mistakes (p. 26).

In Chapter 2 of this report the capabilities of the young child in traffic have been detailed. For many parents and guardians, this information is apparently novel. All too of ten parents assume that a child possesses coping skills which are developmentally beyond his/her ability level. Because the adult is so adept at functioning in traffic, he/she of ten forgets that these skills are not innate to the child. Sandels (1966) has noted:

They (children) do not apprehend the traffic environment in the same way as an adult; they have the grasp of it made possible for them by their age and degree of development. We adults nearly always overestimate children's capacity. We imagine that a situation which we ourselves experience is also the one a small child experiences. In reality, it is never the same. Moreover, the cause of development in early childhood is so rapid that there are great differences not only between adults and children, but also between children of different ages (p. 230). 
Numerous examples have been cited in the literature to support this viewpoint. Pease and Preston (1967), for example, showed that recitation of a safety rule by children did not necessarily represent comprehension.

Likewise, children's misunderstanding of traffic signs and vocabulary is of ten startling to the adults who assume that children share their understanding. The Chairman's Report of Sub-group II of the OECO Special Research Group on Pedestrian Safety (1978) quoted this example from Michalik to illustrate this point: When a six-year-old was asked, "What is a traffic island?" the response was "Where there is water." As Russam (1975) summarizes the situation, "Mothers apparently have a much better opinion of their child's ability to cross roads than research workers do" (p. 31 .

In sum, it appears that parental supervision directly impact on the risks of a child being involved in traffic accident. Moreover, in some of the research it has been found that the quality of the supervision also impacts on the risks to the child. For the parent to be present is not always enough; he/she must be an effective role model for and protector of the child.

\section{I.D. Birth Order: A Survey of the Literature}

Some attempt has been made in the literature to ascertain if there is a relationship between a child's position in the family and a likelihood that he/she will be involved in a traffic accident. Research in this area is, however, quite sketchy. Even among those studies which have explored this relationship, there is a fallure by most authors to present their research methodology or to tie their findings to the accident data. For this reason, we can only turn to opinions of the authors gleaned from observations, rather than concrete data.

Sadler (1972), in her large-scale interview of British mothers, did attempt to examine this varlable in terms of when mothers allowed their children to cross the road. She found that the first-born had been granted 
such permission at a later age than were subsequent children. In this regard, she writes:

... Where mothers said that one of their children did (or would be allowed tol cross at a younger age than the others, the-first crosser was most likely to be the later-born (e.g., the younger of two siblings) (p. 1).

In terms of street playing behavior, Sadler also tried to examine this issue by asking mothers which children in the family played in the street. This question yielded few meaningful results, however. As Sadler observes, "Any differences which appear to be related to birth order may in fact be due to family size as 'oldest' and 'youngest' children must come from families with at least two children and 'middle' children from families with at least three children" (p. 20).

Again, it must be noted that Sadler's data give us information on children's behavior only; there was no attempt by the author to relate these data to accidents. We can, however, surmise that British mothers keep their first-born children out of the road until a later age than they do their subsequent children.

In Japan, Okamoto (1978) observed a similar child-rearing pattern. Although no information is given on how his observations were arrived at, the author offers this conclusion: "The first child is less liable to accidents than the second and subsequently born children" (p. 123).

In Sweden, where there has been some attempt to more rigorously examine this issue, different conclusions were drawn. Ekstrom, Gastrin, and Quist (1966), in examining records of children injured in traffic accidents from 1963-1965, found that first-born children, rather than being safest in traffic, were more likely to be the accident victims (i.e., in $44 \%$ of the cases, child number one was the accident victim). When the percentage of second-born children injured was added to the first-born injured, it accounted for nearly $80 \%$ of all child accident victims. It should be noted, though, these figures may be somewhat misleading in that $59 \%$ of the families whose case historfes were examined had but one or two children in them. 
In the United States, there has been virtually no research done which investigates this variable. The foreign research, as has been seen, is inconsistent in its findings. Therefore, at this point in time, it is not possible to draw any firm conclusions concerning the relationship of birth order to accident probability.

\section{WHERE IS THE ACCIDENT LIKELY TO TAKE PLACE?}

In the above section, literature on the biographic and demographic background of the child pedestrian accident victim was reviewed. In this section where this "typical" victim is most often involved in a traffic accident, will be examined. In this regard, the literature is consistent in its findings on both the probable location site and the child's familiarity with that site.

There is an overwhelming amount of evidence to suggest that most preschool children are injured in the vicinity of their own home, often on the very street on which they live. Internationally, the picture appears to be the same. In Britain, for example, Preston (1972) concluded from her study of 980 accidents in Manchester and Salford in 1969 that "The majority of injuries to children under 5 occur within 100 yards of home, and it is not until children are 11 and over that the majority of injuries occur more than $1 / 4 \mathrm{mile}$ from home" (p. 325).

In another TRRL study, Grayson (1975a) found in an analysis of 431 child pedestrian accidents in Hampshire, that $56 \%$ of children aged four and under were injured within 100 yards of their home. His figures provide the following information on the location of accident sites for children by age (p. 4): 
Distance from Home by Age (Percentages)

\begin{tabular}{|c|c|c|c|c|}
\hline \multirow[b]{2}{*}{ Distance } & \multicolumn{4}{|c|}{ AGE } \\
\hline & ALL & $0-4$ & $5-9$ & $10-14$ \\
\hline $\begin{array}{l}\text { Less than } 100 \\
\text { yards }\end{array}$ & 35 & 56 & 36 & 19 \\
\hline $\begin{array}{l}100 \text { yards to } \\
1 / 4 \text { mile }\end{array}$ & 25 & 17 & 27 & 24 \\
\hline $\begin{array}{l}1 / 4 \mathrm{mile} \text { to } \\
1 / 2 \mathrm{mile}\end{array}$ & 19 & 12 & 19 & 23 \\
\hline $\begin{array}{l}1 / 2 \text { mile to } \\
1 \text { mile }\end{array}$ & 8 & 5 & 8 & 12 \\
\hline Over 1 mile & 13 & 10 & 10 & 22 \\
\hline $\begin{array}{l}\text { Total } \\
\text { Base }\end{array}$ & $\begin{array}{l}100 \\
431\end{array}$ & $\begin{array}{r}100 \\
83\end{array}$ & $\begin{array}{l}100 \\
244\end{array}$ & $\begin{array}{l}100 \\
104\end{array}$ \\
\hline
\end{tabular}

Grayson summarizes as follows:

These results show clearly the increase in distance from home with age, but it is also the case that even in the oldest age group two-thirds of those injured were within half a mile of their homes. Overall, three out of five of the injured children were within 1/4 mile of their homes when the accident occurred, and at least a quarter of these were actually in the street where they lived, according to supplementary information on the accident report $(p, 4)$.

As may be inferred from the above quote, Grayson found that an overwhelming majority of children were familiar with the road on which they were injured. Only $6 \%$ of the entire sample of children claimed never to have crossed the road before. Indeed, nearly $50 \%$ of the children reported that they crossed the road in question daily. 
Foote (1974) likewise found that children involved in pedestrian traffic accidents in Britain were familiar with the location site of their accident. In a paper presented before the 12 th International Study Week in Traffic Engineering and Safety, he proclaimed that $84 \%$ of children under ten in Great Britain were injured within 800 meters (i.e., $1 / 2 \mathrm{mile}$ ) of their home. Bartholomew (1967) had, a decade earlier, similarly concluded that child pedestrian accidents occur within one block of the child's home.

In Japan, the same picture emerges. Hoshi (1976) writes: "Almost all traffic accidents involving young children occur in the vicinity of the child's home. About $75 \%$ occur within 100 meters and $85 \%$ within 500 meters of the child's home" (p. 14). Okamoto (1978) concurs with Hoshi's profile noting that most accidents to young children occur in the road in front of their home.

In the Netherlands, Foote (1974) reiterates the same position. The author goes on to state that " $70 \%$ of all accidents involving children under six do not happen on major roads, but in streets that carry less than 300 cars per 24 hours. It is in the residential areas proximal to the child's house that the accident is most likely to take place" (p. 10).

Evidence from Sweden presents the same description. Sandels' (1975) analysis of her many studies has led to this conclusion: "The analysis leaves the impression that the typical collision between a walking child and an automobile happens on a suburban road, eventually a medium size road...and starts with the child crossing the road unexpectedly" (p. 80).

Studies in the United States have likewise confirmed this pattern. In the ORI study (Snyder \& Knoblauch, 1971), it was found that almost one-half of the accidents analyzed occurred in residential areas. Seventy-eight percent of accidents occurred on two-way streets on which the crossing differences were under 40 feet. It should be underscored, though, that the accident pattern summarized above applies to all accidents examined in the study, not just the $10 \%$ that occurred to children age four and under. 
The study of urban accidents by Knoblauch (1975) in 1972-1974 revealed a nearly identical pattern. For the $9 \%$ of the children in the study who were under four, $77 \%$ were injured in a residential area and $78 \%$ on a two-way road.

In another survey of 1,531 rural and suburban pedestrian accidents in six states, Knoblauch (1977) found that among children aged birth through four, $58.9 \%$ of the children injured were hit within .1 mile of their home. Apparently even in rural areas, the young child is most at risk in his/her own neighborhood.

The U.S. data are so similar to the foreign data that the AAA (1965) has concluded: "Most accidents occur on residential streets within a short distance from the victim's home" (p. 5). The evidence is so significant that it can categorically be stated that the majority of young child pedestrian accidents both here and abroad occur on roads familiar to the child, near his/her home, and very of ten on the very street on which he/she lives.

III. WHAT DOES THE POTENTIAL VICTIM DO THAT PUTS HIM/HER AT RISK?

In regard to this question, the international evidence is equally consistent. It has been determined that the potential victim is likely to be injured near home. The reason he/she becomes involved in an accident is not because of trave1, but because of social reasons. Impulsive and socially oriented, the young child is likely to run out from between parked cars to greet a friend or retrieve a play object. His/her sudden entry in the street is the leading cause of injury.

Sandels (1975), who has spent the last two decades observing the young Swedish child in traffic, writes in this regard:

When a child pedestrian is injured in a traffic accident, the accident is usually entitled a "dashing-out" accident or a "sudden emergence accident." This term designates the accidents in which children are said to have run, jumped or dashed out into the path of the oncoming vehicle. This is always the most common type of accident (p. 13). 
In her research for the Institute of Child Psychology (1974), Sandels attempted to analyze the causes of these dashing-out accidents. In 56 of the 182 cases examined $(31 \%)$, Sandels identified a social factor which precipitated the accident. She writes: "The child ran across the street to somebody, or in front of, or behind somebody, or ran away from somebody he was with" (p. 9). Sandels gives the following two case historles to exemplify this point:

A two-year-old stands next to the road together with some friends. A car comes along moving slowly. When it is about five yards from the children, one of them runs out in front of it. The car stops without hitting this child. The two-year-old runs after the friend on the road and runs into the car, which is then standing still (p.9).

A four-year-old is in his father's car on the way from the doctor's. At home grandma is waiting. The child is anxious to tell his grandmother about the visit to the doctor. While the father locks his car, the child runs away from him across the street (p. 9).

Other "dash-out" accidents in Sweden have been attributed to the fact that the child was so immersed in a game that he/she was oblivious to the traffic on the road, that he/she used faulty judgment in trying to cross the road or the fact that the child thought that he/she could control the flow of traffic by playing policeman or someone else with authority or magical powers.

In other countries, similar reasons have been attributed to the cause of preschool traffic accidents. Hoshi (1976) records that in Japan

"Approximately 70 percent of all traffic accidents involving young children occur when a child dashes suddenly into the roadway" (p. 14). Yoda (1973) has likewise noted that most traffic accidents in Japan are caused by the child suddenly jumping out into traffic. To illustrate, Okamoto (1978) gives this example of a typical preschool accident in Japan:

...when a child on the way home from kindergarten sees his mother waiting for him to cross the street, he attempts to cross the road to join the mother without looking in both directions 
and is run over by an oncoming car. This child may have been repeatedly told to look left and right before crossing and never to jump out into roads. If asked, he would give the correct way of crossing a road. However, upon seeing his mother, he becomes anxious to be with her as soon as possible. Then all precautionary steps to be taken before crossing roads slip out of his consciousness. He can only see his mother and take no notice of oncoming cars. Children cannot size up their environmental situation as adults do. The behavior of small children described above may be called "tunnel vision" (p. 121).

In Britain, analyses of road accidents have produced similar results. Grayson (1975a) in his study of accidents in Hampshire found that most young children injured in traffic were simply not paying attention to the traffic environment. For children under four, a full $82 \%$ were totally inattentive to the traffic situation that led to their accident. Grayson writes: "The most striking feature of this (study) is the very small proportion of accidents which could be attributed to misjudgment on the part of the child... In 90 percent of cases the child reported partial or complete lack of attention. Play or some form of other distraction was associated with over a quarter of the accidents to child pedestrians" (pp. 8-10).

The U.S. research regarding the factors that predispose children to accidents has confirmed the findings of international studies. In an effort to organize accidents into a typology, researchers for the past decade (beginning with the ORI study) have been able to categorize accidents according to a function/event sequence breakdown. For the young child, two types of accidents have been shown to account for the majority of all pedestrian accidents: the dart-out and the midblock dash. The definitions of these categorizations are as follows (Wolfe, 1979; U.S. Department of Transportation, n.d.l:

- Dart-out, first half: A pedestrian, not in an intersection or crosswalk, enters the street midblock and is struck by or walks or runs into a moving vehicle.

- Dart-out, second half: This is like the type above, except that the pedestrian has already crossed half of the road.

- Midblock dash: The pedestrian runs into the roadway, not at an intersection. Differs from the dart-out in that the pedestrian does not appear suddeniy in the path of the vehicle (the driver is usually aware of the pedestrian before the collision is imminent, but misinterprets his/her actions). 
According to the ORI data (Snyder \& Knoblauch, 1971), children under four accounted for $23 \%$ of all dart-out, first half accidents. Another $16 \%$ of all dart-out, second half accidents involved children under four. The statistics on dart-outs stand out all the more in light of the fact that the preschool age group accounted for only $10 \%$ of the accident population sampled.

In a study of suburban and rural accidents (Knoblauch, 1977), a similar picture emerged. Twenty-nine percent of all dart-out, first half victims were under four. Another $16 \%$ of the dart-out, second half accident group was composed of children under four. This same age group accounted for $21 \%$ of the midblock dashes that occurred.

In the study of urban pedestrian accidents, (Knoblauch, 1975) the involvement of young children in dart and dash type accidents becomes even clearer. According to these data, a staggering $44 \%$ of all accidents to children under four were of the dart-out, first half type. While the dart-out, second half accounted for only another $7 \%$ of accidents, the midblock dash was attributed to another $17 \%$ of this age category. Together, these three types of accidents accounted for $68 \%$ of a 11 accidents involving children under five.

This profile is entirely consistent with the portrait drawn by foreign researchers of the preschool accident victim: the young child suddenly emerges into the traffic picture from a midblock position. Play and other social motives have, without warning, placed the chlld at risk in traffic.

\section{WHEN IS THE CHILD ACCIOENT VICTIM MOST LIKELY TO BE AT RISK?}

Another important question discussed in the literature concerns when children are likely to be injured. Again, international data are strikingly consistent in this regard.

\section{A. Time of Day and Accident Causation}

In general, pedestrian accidents involving preschoolers are likely to occur from the late afternoon to dusk hours. Country after country reports this same finding. 
In Sweden, Ekstrom et a1., (1966) report that 50\% of all child pedestrian accidents in stockholm occur in the 2 to $6 \mathrm{p.m}$. time frame. Mollstedt (1966) comments that this afternoon peak coincides with the time when all children's accidents are likely to occur. He writes, "As for the time of day when accidents occur, it is a well-known fact in occupational medicine that most accidents take place in the afternoon, say, between 2 and $6 \mathrm{p} . \mathrm{m}$., which corresponds fairly well with the peak of children's accidents in Stockholm" (p. 162). Lindstrom (1966) reports nearly identical statistics in Gothenburg as in Stockholm: $51 \%$ of child pedestrian accidents occur from 2 to $6 \mathrm{p} . \mathrm{m}$. Sandels (1975) has found that the typical preschool traffic accident occurs around $5 \mathrm{p} . \mathrm{m}$.

British reports are similar. Grayson's Hampshire study (1975a) found that "The peak period for child pedestrian accidents was between 4 and 5 p.m., when more than one-fifth of the accident victims were injured" (p. 4). Foote (1974) found an even greater proportion of accidents occurring during these hours. His research showed that some $60 \%$ of children under age ten in Great Britain were injured between 3 and 7 p.m.

In New South Wales (Avery, 1974), it was reported in 1972 that $67 \%$ of child pedestrian casualties occurred between the hours of 3 to $6 \mathrm{p} . \mathrm{m}$. In Vancouver (Read et al., 1968), it was established that $79.8 \%$ of child pedestrian accidents occurred in daylight.

The data in Japan are again similar. Hoshi (1976) reports that the majority of traffic accidents involving young children occur between 2-6 p.m. Okamoto (1978) echoes Hoshi: "In the case of children, more accidents occur in the afternoon and evening than in the morning. The most dangerous time for children is from 3 to 6 p.m." (p. 123).

International surveys have produced consistent results. Nordentoft (1974) notes that most accidents occur at 5 p.m. Likewise, Magnusson's (1966) survey of ten years of child pedestrian accidents reported in the Index Medicus revealed that most accidents occurred from 4 to $6 \mathrm{p} . \mathrm{m}$. 
As already noted, an analysis of U.S. accident reports is entirely consistent with the above analysis. As one research report summarizes (Wolfe, 1979): "There is widespread agreement that pedestrian accidents peak late in the afternoon--usually between 4:00 and 7:00, or sometimes between 3:00 and 6:00" (p. 5). Huelke and navis (1969) put the probability of child pedestrian accidents occurring in the 3 to $6 \mathrm{p.m}$. time slot at $50 \%$.

National studies of children's accident patterns have yielded similar results. The ORI study (Snyder \& Knoblauch, 1971) showed that the majority of traffic accidents for children under five occurred from 4 to $6 \mathrm{p.m}$. In the Urban study (Knoblauch, 1975), some 84\% of the accidents involving children under age five took place in daylight hours. Overa 11 , some $53 \%$ of accidents occurred in the 2 to $4 \mathrm{p} . \mathrm{m}$. time bracket. The results of the RUPED study (Knoblauch, 1977) indicated that this same time period left child pedestrians most vulnerable. Forty-nine percent of the accidents on this data base occurred between 2 to 4 p.m. as well.

In sum, the results of the data reviewed indicate that the young child is most likely to be at risk in traffic during the hours from late afternoon until dusk. Several explanations have been put forth to interpret this phenomenon. First of all, these hours correspond to the afternoon rush hour traffic peak in all of the countries surveyed. With more vehicles on the road, it is only logical to assume that the risks of the child being injured will correspondingly increase. However, the fact that the morning traffic rush hour does not impact on the child's safety confirms what has been said earlier: children do not seem to be involved in accidents because of travel, but because of play and other social activities. It is not the trip to and from preschool/day care/kindergarten which is most dangerous. Rather, it is the child's play activities in or near the street coupled with his/her social needs that place the child at risk.

\section{IV.B. Day of the Week and Accident Causation}

International research has not been as thorough in analyzing accident data relevant to when in the week accidents are likely to occur as it has in regard to time of day. Still, there are sufficient data from which to make some limited comments. 
Okamoto (1978) reports that in Japan. Thursday, Friday, and Saturday are the most dangerous days to youngsters. Sunday is considered to be the safest day since it is "family day." As such, it is felt that parents are more attentive to their children and thus, better supervisors of their activities.

Tanaka (1965) found that for children who went to nurseries, Mondays and Saturdays were the most dangerous days. He has linked the accident rate to the presumed mental and physical fatigue of mothers at the start and finish of the week.

In Cameron et a1.'s (1976) study of Sydney, it was found that friday and Saturday were the most dangerous days and Sunday the safest.

In Sweden, Sandels presents this analysis of the Skandia I data:

The majority of the accidents occurred during the period Monday to friday. The greatest number of accidents occurred on Fridays but the differences between the days were relatively small. The fewest number of accidents took place on Sundays, followed by Saturdays. No differences with respect to sex have been observed in this connection (p. 11).

In the U.S. more attention has been placed in establishing day-of-the-week accident findings than has been focused on this area abroad. In the ORI study, looking only at the under four age group, the highest rate of accidents (17. 1\%) take place on Monday. The next most dangerous days are Friday and Thursday. Sunday is by far the safest day. The table below presents the percentage of accidents by day of the week for this age group (Snyder \& Knoblauch, 1971): 


\begin{tabular}{|l|c|}
\hline \multirow{2}{*}{ Day of Week } & Age 0-4 \\
\cline { 2 - 3 } & \% of Accidents Occurring \\
\hline Sunday & 9.7 \\
\hline Monday & 17.1 \\
\hline Tuesday & 12.0 \\
\hline Wednesday & 14.8 \\
\hline Thursday & 16.2 \\
\hline Friday & 16.7 \\
\hline Saturday & 13.4 \\
\hline
\end{tabular}

The Urban data base (Knoblauch, 1975) showed Wednesday, Friday, and Saturday to be the most risky days of the week, with each day sharing $16 \%$ of the total number of accidents incurred. It must be noted, though, that this analysis reflects accident data from all age groups and not just the preschool population.

In the RUPED data (Knoblauch, 1977), there was a fairly even distribution of accidents across all days of the week. Thursday and friday each accounted for $16 \%$ of the accident total. The lowest percentage on a given day, however, never went below 13\%; Sunday, Tuesday, and Wednesday all shared this distribution. Again, though, these findings must be tempered by the fact that this breakdown is for all pedestrians, not just the preschool population. 
In sum, there are not clear-cut demarcations concerning the day of the week during which the preschooler is at greatest risk. The beginning and ends of the work week appear to be particularly dangerous times, but again, these findings are not conclusive. There is also some indication that Sunday may be the safest day for the young child.

\section{IV.C. Season of the Year and Accident Causation}

The final variable to be explored in this review concerns the time of the year which is most dangerous to the child. Again, data in this area are somewhat sketchy.

In Sweden, Sandels (1975) has, however, examined this question. Unitike cycling accidents for which there is a seasonal pattern, pedestrian accidents involving young children appear to be evenly distributed throughout the year. Sandels did, however, note a rise in accidents during the months of May and October.

In Japan, Tanaka's (1966) study of nursery children showed September and october to be the most dangerous months. The author attributes the rise in these months following the relatively safe summer to the fact that "this may be due to children's increased outdoor playing after heat in the summer has gone" ( $p$. 33). April, the month when the nursery begins, is thought to be among the safest because of the extra precautions taken by parents at the start of the school year.

Read et a1., (1963) from their study of Vancouver in 1958-1960, found that most child pedestrian accidents occurred in June and September; January and July were the least dangerous months. August and December were also relatively low accident months.

Nordentoft (1974) has concluded that most child pedestrian accidents occur in the spring and autumn. Less accidents, according to the author, take place in the winter because children are more of ten indoors and not as exposed to trafic. 
Magnusson's (1966) review of accident cases listed in the Index Medicus found that most accidents occur during the summer months of April through September.

In the U.S. approach to this topic, there has been some attempt to examine the relationship between accidents and climatic conditions. Using the ORI data base (Snyder \& Knoblauch, 1971), Thackray and Dueker (1983) showed that for children under age five, $84 \%$ of all accidents occurred at temperatures above 45 degrees Farenheit. Looking at all ages on the ORI data tape, Apr 11 was the most dangerous month, followed by May, June, and July. Accidents fell off sharply during September and October.

From the study of urban accidents (Knoblauch, 1975), a somewhat different picture emerges. February through october are the highest accident months, with March being the most dangerous. August, November, and December showed the lowest accident rate. This information, it should be noted, applies to the entire age span surveyed by the study, not just the preschool group. Moreover, no differentiation has been made between climates in northern and in southern cities.

Using the RUPED sample (Knoblauch, 1977), accidents appear to be evenly spread throughout the year. July, August, and october are the highest accident months, with $9 \%$ of the total number of accidents each. February, with $6 \%$ of the total, is the month with the fewest number of accidents. Again, these figures incorporate all age groups and do not demarcate northern city statistics from southern ones.

In sumarizing the literature on this topic, it is difficult to draw any definite conclusions. Based on the foreign statistics and the one U.S. study for which there is preschool-linked data, it appears that accidents involving young children peak during warm weather. It is logical to assume that as the weather becomes inclement, parents would tend to keep their children indoors more. Thus, during the cold winter months in the North, accident rates most probabiy go down. With the renewal of the warm weather in the spring, it appears that accident rates increase in accompaniment to the rising temperature. 


\section{v. SUMMARY}

In this chapter of the report an overview of the "typical" preschool pedestrian accident victim has been presented. Who the pedestrian accident victim is, where accidents are likely to take place, what factors are associated with these accidents, and when these accidents are most likely to occur have been examined. Based on the cross-cultural literature reviewed, the following conclusions can be stated:

- More preschool boys than girls are involved in pedestrian traffic accidents. In many counties, twice as many boys as giris are accident victims.

- Exposure theory does not adequately account for the predominance of boys over girls in the accident data, although childrearing studies indicate that more mothers allow their sons to play in the street than they do daughters.

- The behavior of young boys in the street (e.g., impulsive, risk-taking action) appears to increase the chances of their being involved in an accident.

- The absence of parental supervision is associated with accident involvement. There is some indication that the quality of the supervision is an important predisposing factor in accident causation.

- The research on the relationship of birth order to accident probability is both limited and inconsistent.

- Young children are injured on roads with which they are familiar. Quite often the accident site is the very street on which they live.

- Most accidents are caused by the sudden darting or dashing out of children into traffic from a midblock location. Play and other social reasons--not purposeful travel--predispose the child to accidents. The basic error children make is to not adequately stay and search for oncoming traffic before entering the street. The driver does not have sufficient time to avoid a collision.

- Most child pedestrian accidents occur in the late afternoon to dusk between the hours of 3-6 p.m.

- There is not clear-cut evidence as to which day of the week is most dangerous, although most studies show the beginning and ending days of the work week to have the highest accident rates. Sunday appears to be the safest day of the week for the young child in traffic. 
- There are not clear-cut data on which seasons of the year are the most likely to produce the most accidents. Evidence indicates that accidents involving young children peak during the warm weather and decrease during the winter months in those places where the temperature falls below $45^{\circ} \mathrm{F}$.

Taken together, this information provides us with a wealth of biographical data on the potential preschool traffic accident victim. 


\section{2}

\section{DEVELOPMENTAL CAPABILITIES OF YOUNG CHILDREN}

The young preschool child is developmentally immature. Cognitively, motorically, physically, and emotionally the child is in the process of growing and developing. What this means is that the child--unlike the adult--possesses capabilities which are not fully developed. His/her thought processes are not yet capable of dealing with the abstract. His/her tendency is to focus on one specific feature in the environment, which is by no means necessarily the most important one. For the young child in traffic, the fact that he/she is developmentally still growing may place him/her at risk. Without fully developed visual, auditory, and perceptual skills, for example, the child is handicapped in his/her ability to cope with traffic. These precise developmental handicaps are the focus of this chapter. The chapter begins with a developmental overview of the preschool child, aged two through five.

\section{DEVELOPMENTAL PROF ILE}

The young child aged two through five is at a stage in his/her development where the immediate and the apparent absorb the child's attention. To theoreticians who have attempted to describe this stage of development in terms of qualitatively maturing cognitive structures, the young preschool child is at a point where symbolic imagery is becoming the focus of his/her attention. Piaget (1952) terms this point in the child's development "preoperational"; Bruner (1973) has named this stage "iconic." 
The young child, at this point in life, is suddenly aware of a world of symbols. Language, play, and dreams all reflect this newfound cognitive growth. To the preschool child who has heretofore been confined to his/her immediate space and present perceptual situation, symbolic functioning ${ }^{\prime}$ can open up new worlds of exploration.

Through the acquisition and utilization of language, the child can create a word to stand for something even when that object is not present. At first, a child may pick up a stone and declare that it is an insect. Should, however, someone come along and walk on the stone or cast it aside, the child will most likely be upset that someone has injured the insect (Elkind, 1974). Slowly, the child begins to gain an understanding that words and symbols can be distinguished.

Through play, the child becomes absorbed in symbolic images. For the first time, imaginary play is experienced. The child may put together blocks and show the parent the rocket ship that has been produced. Or the child may create a friend named Ralph with whom the child can talk over the events of the day.

Also, for the first time, children report the occurrence of dreams and nightmares. Dreams, like other symbolic images for the child this age, appear to exist on their own. To the child, the dream is real and the events depicted in the dream happened just as dreamed, even though the dream may contradict known truths. The line between reality and dreams slips back and forth.

While this new stage of symbolic thought is exciting and growthenhancing, it poses many pitfalls for the child in terms of what we would call rational thinking. As Elkind (1974) indicates: "...This new capacity for representation which loosed the infant from his egocentrism with respect to objects, now ensnares the preschool child in a new egocentrism with respect to

1/Symbolic functioning (Piaget, 1962) refers to the child's ability to create a mental image to stand for something that is not present. 
symbols (p. 76)." For the young child who isgrowing and testing the environment, these limitations are but a step in the process of learning and not a problem but a learning challenge. But to the young child exposed to the dangers of traffic, these limitations are indeed a problem--and of tentimes a danger. These limitations that impact on the child in traffic are briefly summarized in the following sections.

\section{I.A. Egocentrism}

Preschool children are characteristically egocentric. To the child, everything in the environment is viewed from his/her perspective. According to Piaget and other theorists, the child is usually incapable of understanding another's point of view since he/she believes that everyone shares his/her own point of view. Piaget (1954) describes the child's perspective on the wor 1d in this way:

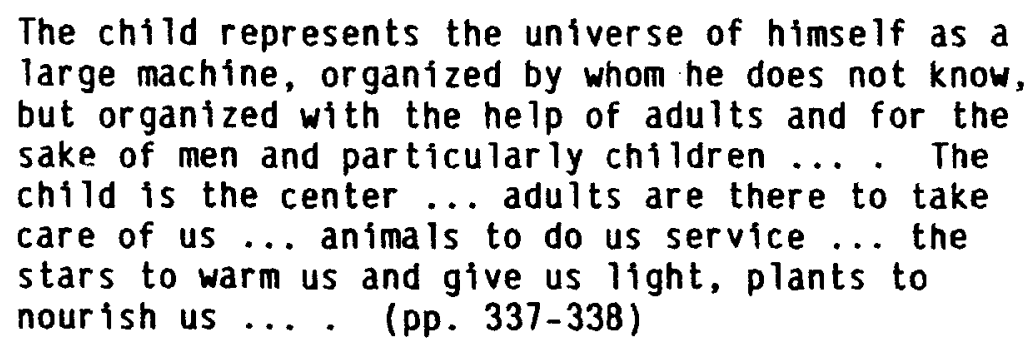

The egocentric child is placed at risk in traffic. Because he/she thinks that the world centers around $\mathrm{him} / \mathrm{her}$, he/she does not feel a need to assess the traffic situation before running across the street. The environment is there to serve him/her. Likewise, the child is not concerned with what actions the driver of an approaching car might take. The driver is there to serve him/her as well.

\section{I.B. Transductive Reasoning}

The thinking of the young child differs radically from that of an adult. Whereas adults tend to think either inductively lgoing from the specific to the general) or deductively (going from the general to the specific), young children do neither. Their thought is described as transductive, i.e., going 
from the specific to the specific. The child, it seems, makes no attempt to link any specific thought to a generality. Piaget (1951) gives this example of the way transductive reasoning operates:

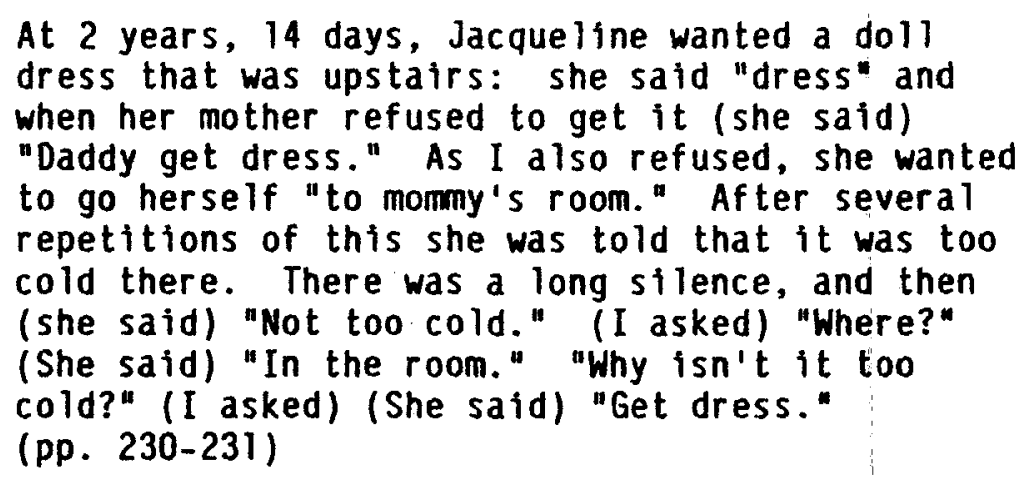

Transductive reasoning, it has been noted, occurs when children attempt to assume causality between events when none exists. For the child in traffic, this means that the youngster may not understand that a driver has to see him or her before stopping the car. To the child, actions do not grow out of causes, so the toddler cannot make judgments about how to act safely when crossing the street.

\section{I.C. Centering}

As the preschool child is working at the tasks of reasoning, he/she learns how to center, i.e., to concentrate on a single outstanding characteristic of an object while excluding all other features. While this process is an important step in learning how to think intuitively, it has the potential for handicapping the child's perception of an object. Centering of ten leads to confusion when the child excludes an important feature from his/her perception. This example from Richmond (1971) illustrates:

In an experiment the child is given 20 wooden beads, 18 of which are brown and two of which are white --

ADULT: Are there more wooden beads or more brown beads?

CHILD: More brown ones, because there are two white ones.

ADULT: Are the white ones made of wood?

CHILD: Yes.

ADULT: And the brown ones?

CHILD: Yes.

ADULT: Then there are more brown ones or more wooden ones?

CHILD: More brown ones. (p. 38) 
The single-mindness on the part of the child can place him/her in a precarious position in traffic. There is no guarantee that the object of the child's attention in crossing the road will be one that is relevant to his/her safety. It is perfectly likely, for example, that the color of an approaching car will be of more interest to the child than the fact that it is moving toward $\mathrm{him} / \mathrm{her}$.

\section{I.D. Inability to Conserve}

Conservation, according to Piaget (1970) is the ability to recognize that a quantity remains the same even though its form might change. This concept of "permanence within apparent change" (Elkind, 1974) applies to weight, substance, length, number, volume, and space.

Piaget has performed many classic experiments that illustrate the young child's inability to conserve. In one example, a child is presented with two equal size balls of clay and asked if they are the same size. The child concurs that they are. The experimenter then takes one of the clay bal1s and in front of the child rolls the ball into a sausage shape. The child is then asked to examine both objects of clay to decide if they are the same size. This time the child will report that one of the objects is larger than the other. If the child is then asked if any clay has been taken away or added from the original two balls, the child will say "no." The difference, the child will note, stems from the fact that one of the clay forms is longer, wider, or taller.

Inherent in the child's inability to conserve is his/her lack of understanding of the concept of irreversibility. Preschool children are not as yet able to understand that every logical operation is reversible. Phillips (1969) gives this further illustration of the child's lack of understanding:

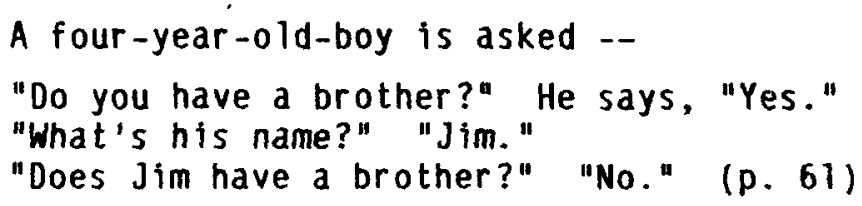


Again, the child's limited reasoning powers may pose a threat to him/her in traffic. Unable to conserve or to understand the concept of irreversibility, the child faces added.risks. The young girl spying an approaching car in the distance may not understand that the small car which seemed static is actually advancing toward her as it grows larger in size. Or a child may look at two parked cars and realize that they are the same size. When one car leaves the parking spot, though, and backs out behind the still-parked car, the youngster may no longer perceive the car as being as large as he first thought it to be.

\section{I.E. Distortion of Reality}

As already noted, the young child who is just beginning to grasp the concept of symbolic functioning of ten drifts in and out of a world of fantasy. Stones may be insects, blocks may be rockets, and dreams are fact. Nothing to the young child is inner or subjective; all thoughts, dreams, and deeds share a common reality.

For children in traffic, this poses a tremendous danger. The child who is playing a game may incorporate the approaching car as an object in his/her game. Or the child who plays policeman may think that he/she has the power to stop traffic. The dangers of traffic are not any more real to the child than the fantasy in which he/she is presently engaged.

Taken together, it can be seen that the preoperational child is limited in his/her capacity to understand and cope in traffic. The symbolic functioning which characterizes his/her thoughts, puts him/her in a precarious position in traffic. In the remainder of this chapter the limitations that are placed on the child in traffic because of his/her developmental immaturity are explored.

\section{REVIEW OF SPECIFIC CAPABILITIES OF PRESCHOOLERS}

In this section the relevant capabilities of young children to deal directly with the traffic system, and their ability to learn how to interact with this environment are reviewed. While few capabilities can be classified exclusively as one type or another, somewhat arbitrarily, vision, audition, 
attention, spatial relations, distance, velocity, causality, motor behavior, reaction time, hazards/risks and short-term memory have been targeted as topics dealing primarily with the traffic environment, while rules, left/right, vocabulary, traffic signals, communication, logical reasoning, logical operations, crossing judgment, route planning, and long-term memory are dealt with as topics dealing primarlily with educational concerns.

\section{TRAFFIC RELATED CAPABILITIES}

II. A. Vision

Specific visual perceptual capabilities place the preschool child at risk in traffic. During the first few years of life the visual system undergoes impressive changes. Although certain parts of the eye, like the lens, continue to grow through the life of an individual, most of the ocular structures are complete around age seven. Throughout this period the young child is not only learning to perceive the surrounding environment but is also learning to perceive it while the physical structures of the eye are changing.

The growth of the eye is closely related to the growth of the brain (Warwick, 1976). From birth to maturity, the eye grows 3.25 times its initial size while the brain increases 3.76 times. The body itself will increase an impressive 21.36 times. Because the young child's eye is shallow, he/she is typically farsighted. As the eye becomes more spherical in the first several years, the lens, which focuses light on the retina, flattens as it is pulled into an ever-widening circle by the ciliary body. These specific changes are responsible for the development of the capability of accommodation, the process of focusing the image by adjusting the thickness of the lens.

Until recently, behavioral measures of young children's visual acuity estimated that aduit efficiency developed anywhere between two to seven years of age (Newman, 1975; Illingworth, 1975). These tests showed that the acuity of preschool children fell in the range of 20/200 to 20/400 (where Snellen $20 / 20$ corresponds to the normal adult ability to resolve one minute of visual arc). The difficulties involved in testing young children led some 
researchers to turn to psychophysical techniques in order to circumvent the problems inherent with behavioral tasks for children. These techniques have significantly lowered the age of visual acuity efficiency as occurring at two to six months of age.

The most promising technique uses Visually Evoked Responses (VERs), also called Visually Evoked Potentials, to chart the development of children's visual capacities. The VER is an averaged record of cortical activity that occurs when an individual views a visual stimulus. It is assumed to reflect the activity of retinal receptors, particularly the faster response latency of the cones (Berg \& Berg, 1979). Electrodes are placed on the occipital cortex to pick up positive and negative potentials which are averaged over many trials by a computer to yield a wave form response. The VER can be used to measure the eye's sensitivity to different spectral wavelengths (hues) and to measure visual acuity as well.

Harter and Suitt (1970) traced the changes in. VERs in one infant to differently sized patterns beginning in the first month of life. From the thirty-fifth day on, successively smaller check sizes elicited the greatest response until an adult's inverted $U$ pattern appeared at about 2.5 months with a peak corresponding to that found in adults with $20 / 250$ acuity. The same technique was used by Sokol and Dobson (1976) with 15 infants ranging in age from two to six months. They found that the peak response decilined with age and became nearly identical to the inverted $U$ pattern of $20 / 20$ adults by the age of six months.

Instead of matching infant functions to adult ones, Marg. Freeman, Peltzman, and Goldstein (1976) measured the threshold activity of infants directly by eliciting optokinetic nystagmus (i.e., an involatory jerking movement of the eyeball caused by looking at moving objects/ to bar gratings of various sizes. When gratings are very small, they appear to form a homogeneous gray field. As they become larger; however, a point is reached where the bars become individually discernable and, if they are made to appear to move, will elicit reflexive tracking eye movements. The threshold is taken as the highest spatial frequency (smallest sized bars) that elicits a response 
clearly different from that of the gray field. Infants showed rapid improvement in the first two months and reached adult levels by four to five months.

These psychophysically based studies indicate that the visual system is better developed and at a substantially earlier age than previously thought. Although specific ocular structures continue to grow throughout the preschool years, the primary receptor portions are established during the first 6 months. Continuous refinements will be made during the following years as the child learns to deal with the visual cues provided by his or her growing system.

Numerous studies show that children have a difficult time when it comes to choosing the key elements in their visual world (Day, 1975; Walk, 1978). Very young chlldren rarely scan the visual field in a meaningful way; in fact, infants usually show a very disjointed pattern of inspection. By age six, this awkwardness gives way to scanning that focuses mainly on the highly informative parts of a figure searching these informative areas more quickly, efficiently, and thoroughly.

The scanning patterns of three-, four-, five-, and six-year-old children were photographed by Zinchenko, Van Chzhi-tsin, and Tarakanov (1963) while the children inspected patterns that they were asked to either familiarize themselves with or to identify. The youngest children showed very little activity during familiarization, since their fixations tended to cluster around the center of the pattern where the camera lens was located. The four-year-olds began to link up fixations around aspects of the figure and to use sweeping movements covering the entire surface as well. The five-year-olds continued these trends except that they examined the salient features more completely and systematically. The eye movements of the six-year-olds followed the entire contour in an exhaustive exploration of the most informative aspects of the figure.

When it came to the recognition task, however, these patterns changed. The three-year-old children made large sweeping movements that of ten led them beyond the figure's contours while the four-, five-, and six-year-olds were 
content to fixate only a limited number of features. As the children increased in age their search time decreased, the number of eye movements increased, and the number of identification errors was reduced. Zaporozhets (1965) confirmed these results.

The scanning strategies used by three-, five-, six-and-a-half and nine-year-old children who were asked to make same or different judgments of windows in two houses were investigated by Vurpillot (1968). Some of the six windows in each house had curtains, flowers, or other distinguishing features while others did not. The children used markedly different strategies that led to different success rates. The youngest children looked at only 6 or 7 of the possible 12 windows before giving their judgment regardless of whether the houses were the same or different. The older children, on the other hand, were $a b l e$ to adapt their strategies to the task. When the houses were the same they inspected all of the windows. When there were differences, however, these children ceased their inspection when they located the difference. Since the two older groups performed very similarly, this suggests that adult efficiency is acquired at the earlier age.

The scanning techniques used by six-year-old children was compared to those of adults by Mackworth and Bruner (1970). When adults needed to comprehend a picture they usually used horizontal eye movements and lingered at each fixation longer. If the picture was blurred, adults often developed vertical movements especially during the period when they were trying to identify the picture. Children, on the other hand, never used vertical movements. Their more numerous eye movements were shorter and most importantly, they were inconsistent in the way they scanned a picture. They rarely returned to the same features on subsequent inspections. Whereas contour effects were unimportant for adults, some children concentrated on the outlines of well focused pictures particularly if there was a high contrast patch of color.

Visual scanning patterns used by young children show other regular departures from those of adults. Children rely almost solely upon central foveal vision without taking in peripheral information. Whereas adults take advantage of this source to guide future eye movements, children typically do 
not begin to utilize this source of gross visual information until they begin to read (Gibson, 1969; Rosinski, 1977). This tendency has been called "tunnel vision" (Yoshiyuki, 1978; Miller, 1969).

There is a steady decline in the length of children's fixations with age in addition to the rise in the number of eye movements. Fixation duration is assumed to represent the time taken to process a given piece of information (see Day, 1975). In the Mackworth and Bruner (1970) study six-year-old children very closely resembled adults in this category.

Finally, visual scanning is influenced by the degree of familiarity with the figure. Initially, the number of eye movements increases but then they decline. Whereas the three-year-old children in the Zinchenko et al. (1963) study initially made very few excursions from the center, familiarization with the picture increased their eye movements until they were approximately equal to the number made by the six-year-olds. Subsequent presentations of a stimulus enhances eye movements only up to a certain point, after which there is a decline with additional viewing.

The most important aspect of perceptual learning according to Eleanor Gibson (1969) is specificity. "The criterion of perceptual learning is thus an increase in specificity. What is learned can be described as detection of properties, patterns, and distinctive features" (p. 77). Early data presented by Gibson in support of improved discrimination skills with experience rely on artifically constructed stimuli like 'scribbles' which differ in the number of specific transformations or distinctive features. Children in the four to eight year old, age range consistently make more identification errors and improve less with practice than do older children and adults. Improvement occurs because the distinctive features which allow discrimination between objects are learned through experience.

Evidence from electrophysiological studies of single unit recordings in cats, rabbits, and monkeys show that the visual system has built in feature detectors (see Barlow, 1972). The painstaking research of Hubel and wiesel 1959, 1962, 1965a, 1965b, 1968, 1970) has indicated that the specificity of receptive fields at the retinal, ganglion, and cortical levels is the norm, 
not the exception. Receptive fields have been found which respond to particular arrangements of stimulation like bars, straight edges, dark circles, light circles, and to stimulation from either eye or from both. Some are so specific that they will not register a stimulus unless the orientation is precise.

Experience can alter the normal neural organization. In a series of experiments Hubel and Wiese 1 (1965a) and Wiesel and Hubel (1963, 1965) found that abnormal visual experience such as unilateral eye closure, surgically induced strabismus, or alternating monocular occlusion, which prevents the simultaneous use of the eyes, produced an abnormal group of cortical cells. Indeed, abnormal experience was the most powerful if it occurred during a critical period of development extending from the third to twe if th weeks in cats. Furthermore, kittens who were reared with the two eyes exposed to different stimuli, one to vertical bars, the other to horizontal bars, showed an orientation selectivity in the corresponding receptive fields (Hirsch \& Spinelli, 1970). Kittens who viewed only horizontal or vertical bars had no neurons sensitive to the other orientation (Blakemore \& Cooper, 1970).

These neurophysiological findings support the idea of feature detectors embedded in a preexisting neural organization, which can be modified by experience so important features of the environment receive the maximum cortical representation.

Although the retinal and neural mechanisms of color vision are present in the infant, the young child's ability to perceive color as a perceptual dimension is developmental. Over fifty years ago Brian and Goodenough (1929) showed that children under the age of three rarely used color as a basis for categorization. Between the ages of three and six, however, children preferred color to form, but this preference reversed after the age of six when color was less important again.

The ability to understand color terminology affects young children's ability to use color as a differentiating dimension. Cook (1931) found that half of the two-year-olds who could match objects by color could name the colors correctly only $25 \%$ of the time. By the age of six, $97 \%$ of the children 
could match by color while $62 \%$ of them could accurately identify the color. The ability to abstract color as a property appears around the age of three or four years (Vurpillot, 1976). Before that time a child may very well be able to talk about a "blue ball" or to identify a particular blue jacket. If asked to indicate something else that is blue, however, the young child fails. At this point color is inextricably linked to the object itself and cannot be separated from it for a few more years.

Interestingly, there may be sex differences in the use of the color dimension. Kagan and Lemkin (1961) found that children aged three to nine preferred to sort geometric stimuli on the basis of form, not color or size. Size was rarely used. Younger girls were more likely to use color than older girls but there were no differences among the boys. The boys, however, were more apt to use color than the older girls. This trend was very weak, because if the test stimuli eliminated form, but permitted color and size, one-fifth of the children said that they did not know what the answer was. The authors suggest that the sex differences could be attributed to the greater verbal skills of the girls who could attach other labels to the stimuli.

By the time children reach the age of twelve they are adept at using color cues to aid in visual search tasks. Day (1978) found that all of the children in her study between seven and twelve years of age had reduced search times if color cues were provided. The older children benefited the most in terms of both speed and accuracy of identification at a rate approximately double that of the 20 percent improvement of the seven-year-olds.

The Kagan and Lemkin study indicated that very young children do not understand the concept of size. Part of the difficulty stems from changes in the physical structure of the eye brought about by growth (Bower, 1977). An object seen by both an adult's eye and a child's eye will appear smaller to the child because it projects a smaller retinal image in the child's shallower eye. Retinal size alone, however, does not determine size perception. Other sources of information include those important in distance perception, including convergence, binocular disparity, motion parallax, optical expansion and textural occlusion. 
Convergence is the only growth-related source since it is a function of the distance between the eyes and the angle they make when fixating targets at different distances. As interocular distance increases, it necessarily alters this angle. Closely related to convergence is binocular disparity which refers to the different images projected onto the two retinas by the same object. Motion parallax and optical expansion pick up apparent movement information between two objects in the distance by movement of the head or movement of the objects, respectively. Textural gradient information depends upon knowledge of the differences of various textures (rough concrete vs. a smooth apple) and the way these textures change with distance.

Young children have access to these various sources of size information, many of which are independent of growth changes. Do they make use of them? Almost half a century ago, Thrumm (1935) showed that among children two to four and-a-half years of age, those under the age of three could not discriminate among objects on the basis of size. Above this age, however, children indicated a grasp of relative size. The concept of largest was the most successful with a discrimination rate of 88 percent, followed by smallest $(68 \%)$ and finally mid-sized $(48 \%)$.

The size judgments made by young children between three and five can be altered if rewards are closely associated. Lambert and his colleagues (1949, 1953) found that children perceived tokens to be larger if they had been immediately connected to a candy reward than if the token was given long before the reward.

Young children have trouble differentiating the various parts that make up a perceptual whole figure. On the one hand, they will identify an object on the basis of just one of its elements, and on the other, they will judge that two objects are identical because they each have a certain element (Vurpillot, $1968,1976)$. Stimulus properties play a critical role in the problem, for if they favor perception of the whole, then the whole figure is more likely to be perceived. If, however, the component parts are dominant, then the parts will be seen. This latter was the case for the youngest children (four-and-a-half-years of age) in a study by Elkind, Koegler, and Go (1964) 
where $71 \%$ perceived the parts. With each additional year, this percentage declined until at age nine, the age at which $79 \%$ of the children perceived both the parts and the wholes.

Adults can readily perceive both the whole and its configural parts simultaneously. Rand and Wapner (1969) suggest that younger children are unable to do this because they find it easier to abstract just one aspect than to handle the entire complex figure. The eight-year-olds in their study were less able to perceive embedded figures than were older children.

Thus, it is the perceptual processing component rather than the physiological aspect of the visual system which creates perceptual problems for the preschool child. Although ocular structures continue to grow until maturity, the basic functions are well established much earlier than previously thought. The preschool child certainly has access to the visual cues that help us order our environment. The problem is that there is very little order in the way a young child searches the environment for salient features or for that matter, in the choice of which features are the most informative until about the age of six. Although many improvements will yet take place, by this age a child begins to resemble efficient adult patterns in the way he/she abstracts information from the environment.

\section{8. Audition}

Another sensory system which affects the preschooler's ability to safely interact with the street and traffic environment is audition. There are $a$ number of auditory cues which can potentially alert a young child to oncoming vehicles (tire noise, engine noise, etc.) or immediate danger (skidding tires, honking horns, shouts, etc.) if the child is able to detect and process the information appropriately. This section will deal primarily with the detection issue, although the processing issue will be addressed here and in subsequent sections as we 11.

Experimental research on audition indicates that sensitivity to sound is a developmental capability. Approximately 5,000 children from the Pittsburgh area were given a variety of hearing tests which included tests for 
sensitivity (Eagles, Wisnik, Doerfler, Melnick \& Levine, 1963). The researchers found that sensitivity increased through age twelve, and then declined thereafter. This pattern was particulariy true for the lower sound frequencies, although there was a remote possibility that this finding was due to a methodological problem of earphone leakage. Sex differences in hearing sensitivity were also detected, as girls were more sensitive to all hearing levels (except $250 \mathrm{cps}$ ) with the difference peaking at $2 \mathrm{db}$ at $6,000 \mathrm{cps}$. While it is unlikely that such an auditory difference would account for the previousiy reported sex differences in child accident rates, it is one more factor which may contribute to the risk boys face as pedestrians. These researchers also examined left-right ear sensitivity and found differences of less than $1 \mathrm{db}$.

Additional research indicates that the auditory sensitivity of the two ears varies depending upon the type of stimulus. Kimura (1963) examined 145 children aged four through nine. She excluded those children who were hearing impaired or left-handed ( 25 children) and then presented each child with a series of digits via earphones. In each case, different ears were sent different digits simultaneousiy. Children were asked to report all the digits they had heard. While memory could obviously affect the results, it should not account for the fact that the digits presented to the right ear of the children were more accurately reported than those to the left ear. Boys also scored lower than girls at the early ages, but there was no overall sex effect. The findings are interpreted as suggesting that the left hemisphere of the brain is dominant for speech perception by age four, and thus the input to the right ear would be reported better.

In a later study with adults, using both digits and melodies separately, this right ear effect was replicated with digits, and as predicted, melodies were reported correctly more often when presented to the left ear (Kimura, 1964). It has been speculated that this differential sensitivity between the two ears may affect the child's preference for positioning himself or herself in space, i.e., that the child would be positioned to allow the right or left ear to receive particular stimulation (Newman \& Newman, 1978). 
Bower (1974) has suggested a model for auditory localization to explain how a child uses auditory cues to locate objects in space. He states that there are three dimensions of stimulation used in this process: time difference of the onset of stimulation at each ear; phase differences of the sound wave at each ear, and intensity differences in each ear. While adults can and do use these methods to locate sound sources (Stevens \& Newman, 1936), it has not been clear that children do, since the distance between ears is critically important, and infants and children have less such distance than adults. Infants can identify sounds straight ahead, or to the left and right under this mode1, but the other positions are identified by experience. Research designed to test these predictions using infants under six months of age supported Bower's model (Bower \& Wishart, 1973). Further, the results

- indicated that auditory motor accuracy in these infants was less than visual motor accuracy.

Sandels (1975) has conducted research more directly relevant to the traffic situation for children. She asked about the relative ability of children and aduits to locate the position of a complex sound in a horizontal plane, and whether some positions were easier for children to localize than others. Subjects were 40 six-year-olds and 40 adults, with an equal sex distribution; all subjects without normal hearing were excluded. The sound used was that of a car approaching, driving past, and away. It lasted three seconds, and was presented from 1 of 12 loudspeakers surrounding the subject. The subject was to identify the direction of the sound.

Children did not perform as well as adults on this task; they had fewer correct responses and greater error dispersion. The easiest positions (using a clock face with 12 as the straight-ahead position) for children were 12, 11 , 1 and 6 . Adults could also identify $3,4,8$ and 9 . There were no left-right differences overal1, but children, as opposed to adults, made more left-right errors. The most frequent errors were within a 30 degree range left or right of the correct position. As Sandels points out, there are two types of errors which could affect the interpretation of these findings. Children, and to a lesser extent adults, may have incorrectly identified the location of the sound, but pointed to the correct position inadvertently, or they may have correctly identified the sound but pointed to the incorrect location. 
Although the experiment did not account for these errors, it is readily apparent that children do not perform as well as adults, despite the fact that previous evidence (Eagles, et al., 1963) shows that hearing sensitivity declined after twelve years of age. Given their greater hearing sensitivity, it appears that the processing aspect, rather than the sensory aspect, is the limiting factor for children and thus Sande15' research supports Bower's (1974) position that experience plays a key role in the ability to localize sound accurately. Sandels cites other support for this position as we11: Gibson (1968a), who believes that children select different sounds than adults to attend to, sounds which may provide them with new information or which may momentarily attract their attention, but which may be totally irrelevant to their situation; and Sieganthaler and Barr (1970) who also feel that sensitivity is not the issue, but that experience, training, and development of the nervous system result in the better perception of sounds.

While there have been a number of other studies dealing with audition in children, as with vision, most of the work is with infants. Researchers are continuing to demand further research, particularly because the methodology and equipment continue to advance. White (1975) feels that precise hearing levels can now be tested accurately at three and a half years of age, and he also states that children can accurately. localize sounds as young as three, four; or five months of age.

In a previous review of children's capabilities regarding their ability to cope with the traffic system, Avery (1974) withheld judgment regarding evidence for auditory development during childhood. He cited Kidd and Kidd (1966) as providing divided literature and calling for further research. While Kidd and Kidd did note a trend for greater auditory acuity and pitch discrimination with age, suggesting the importance of maturation, they did not include the work by Eagles, et a1. (1963) in their review. Instead they used a number of earlier works. The more recent work indicates both the developmental/maturational aspects of auditory development and the importance of experience as well.

One other study worth citing in this section indicates the irony of the situation in which society has placed many children. There is evidence that 
one of the many skills children need to survive in the traffic situation, hearing, is diminished by the noise from that very environment. For children living in high-rise bulldings near expressways for four or more years, the lower the floor level of their apartment, the lower their auditory discrimination (Cohen, Glass, \& Singer, 1973). The researchers verified that the sound levels were correlated with floor level, with the lower floor levels receiving the most traffic noise. Even controlling for social class level, these effects remained, although somewhat diminished. The apartments had a very restricted SES range and minimal price variation, so it is unlikely these factors affected the results, although traffic noise itself cannot conclusively account for these effects.

Thus, for the population of concern to this effort, birth through five-year-olds, it appears clear that limited auditory powers and limited processing ability place them at risk relative to older children or adults. Since sensitivity peaks at about age twelve, the age range of concern is still far from optimal sensitivity. Further, as Sandels (1975) has shown, even six-year-olds have problems localizing sounds. Even if one compensates for the fact that her subjects could not turn their heads as they might under normal circumstances to aid sound localization, it is highly likely that in the traffic situation preschoolers are still going to lag far behind adults and six-year-olds in this capability, and therefore, additional steps will be required to adequately protect this population.

\section{C. Attention}

Attention is another important capability in terms of children's ability to deal with the traffic situation. Vinje (1978) feels it is important in two ways: (1) while watching out for traffic the child must keep his attention on the task long enough; and (2) during other activities the child must always be mindful of when he/she crosses into the roadway. In his review of the literature Vinje concludes there is "no reason to suppose that the attention span of young children would not be enough" (p. 11) to do the former and then goes on to discuss the importance of selective attention for the latter. We feel it may be more prudent to conclude that children cannot attend to this task successfully unless there is clear evidence to the contrary. Further, it 
will be important to specify the specific age range referred to since the term "young children" could clearly encompass a broad age level. 01der and Grayson (1974) present evidence to point out the extent of such attention failures in a paper presenting a conceptual analysis of the pedestrian task. On the basis of interviews conducted with pedestrian accident victims in England, they report that $70 \%$ of the adults (fifteen years old or over) did not see the vehicle which struck them, and over $60 \%$ of the children did not see the vehicle they collided with. Further, $39 \%$ of all children showed a complete lack of attention by not looking at all before they crossed. For the birth through four ages, this rate was 50\%. O1der and Grayson conclude that lapses of attention, rather than failures of judgment, are the main factors influencing the behavior of both children and adults. Further, on the basis of time lapse films of children and adults and on the basis of the safety gaps that these groups accept, they conclude that the younger age groups are less efficient information processors, since they require more time to reach a decision and act upon it. Thus, both low attention levels and less efficient processing can clearly combine to create a hazardous situation for preschoolers in a traffic environment.

Vinje (1978) speaks of the importance of selective attention for children in traffic, meaning the control of information processing so that sensory input is perceived or remembered better under some circumstances than others according to the desires of an individual (Schneider \& Shiffrin, 1977). Vinje states that selective attention exists from birth on but in a limited state and then discusses the developmental sequences proposed by Wright and viletstra (1975). They believe that by age four children are especially attracted to new stimuli but cannot control their attention adequately. By five years, there is more control, although irrelevant but salient information is still attended to, particularly color, form, novelty, and surprise. By age six or seven children can differentiate between occasions for playful curiosity and situations requiring a systematic search. Only by about eleven years of age is a real focusing upon relevant information and ignoring of irrelevant information found (Wright \& Vlietstra, 1975). Vinje points out how nicely those data coincide with data from Luria (1973) regarding the final level of maturation, at about twelve to fifteen years of age, of the frontal 
zones of the brain, an area which is "beginning to play a more intimate part in complex and stable forms of higher, voluntary attention" (Luria, 1973, p. 2701 .

Wright and viletstra (1975) believe that exploratory behavior is distinct from, but a necessary precursor to and stimulator of, systematic search behavior. They compare their views with White's $(1965,1966)$ model that posits developmental changes in exploratory and search behavior as shifts from associative levels of response to cognitive levels by about five to seven years of age, concluding that their model is compatible with his model. After reviewing dozens of studies dealing with the development of selective attention, they conclude that the long-term development of selective attention is actually a gradual change in the relative frequency of the two modes of behavior (exploratory and search). Further, each major advance in cognitive development depends upon the availability of appropriate and orderly information which, in turn, depends upon the child's growing ability to engage in selective and appropriate information-getting transactions with his/her environment. Thus, as with audition and vision, experience plays an important role in the development of this capability. Further, preschoolers have yet to approach the peak levels in any of these capabilities.

In research more directly related to the traffic environment, Coote (1976) examined what happened to 30 five-year-olds and 30 twelve-year-olds when they were forced to divide attention between a motoric activity (putting matches in a bottle) and a visual activity (watching for flashes of a torch globe). Five-year-olds had considerably more difficulty dividing their attention on this task, implying that the motor activities involved in pedestrian behavior would hinder young children in watching for traffic. Risk (in this case the threat of stopping the game and thereby losing the dollar payoff) seemed to have no implications for traffic-related attention.

Another study examined which aspects of the traffic situation children attend to (Gunther \& Limbourg, 1976). The aspects most of ten attended to were those least relevant for the traffic task, e.g., animals present, children playing, ice cream trucks, etc. 
Although there are a variety of theortes dealing with the role of attention in a child's development, all leave little doubt that it is of major importance (Hetherington \& Parke, 1979). One position is held by enrichment theorists, like Piaget, who propose that each time a child perceives an object he/she learns a little more about it. With axperience, information is added to the existing schema of an object, and it becomes more elaborate and detailed. Sensory input needs to be modified and enriched by information from other schemata. As this enrichment process occurs, the child is better able to discriminate among objects.

Differentiation theorists (e.g., E. J. Gibson, 1969) emphasize sensory input as a rich, not meager, source of information. Rather than enrichment through schema, the child has to attend to, identify, and discriminate features of objects in a complex sensory stream. With experience, the child makes increasingly fine discriminations and also becomes aware of invariants, characteristics of objects which don't change under different conditions. For example, the child will know that a disk is round even if it is viewed from the side.

Flavell (1977) has suggested four developmental changes in attentional processing by children: (1) shifts in control of attention, (2) adaptability, (3) planfulness, and (4) deployment of attention over time. Control is important because all features of an object are not equally important, and it is necessary to attend to the relevant information, not to irrelevant information. Young children can do this for only very brief periods of time. However, they can be trained to selectively attend to relevant features of the environment. Tighe and Tighe (1969), in a study with 144 first-grade children, found those who were given perceptual pretraining (or perceptual experience and verbal labeling trainingl in distinguishing critical features of an object (e.g., color vs. size) before a discrimination test were markedly superior to their untrained peers. Children can also be taught to increase the number of objects they attend to (Pick, Christy Frankel, 1972). Children of two ages (second and sixth graders) compared aspects of objects under two conditions. One group was informed of the relevant aspect beforehand, the other group after the stimulus presentation. Overall the 
older children had faster reaction times, and the difference in reaction times was greatest for the older children as well. The results were interpreted as indicating a developmental trend for greater selectivity of attention.

Adaptability, Flavell's second developmental change, is important because no one attentional strategy is appropriate for all situations. 0lder children are more flexible than younger children in modifying their attention in response to changing task requirements. Planfulness, the third change, involves the readiness to process certain selected types of useful information in accordance with a plan or strategy. This, too, is a developmental attribute. Even though younger children may appear to use planful strategies, they cannot report what they are doing as well as older children (Flavell, 1977).

The final change is the deployment of attention over time, as children become able to process complex sequences of information. This process is summarized as follows:

My own mental image of a cognitively mature information processor is that of a conductor who directs his ensemble of musicians (attentional processes and resources)--now calling for th one instrument, now another, now a blended combination of several or all depending upon the effect desired. I think we do not so much "pay attention" as "play our attentional system." That is, we intentionally exploit and deploy it in a flexible, situation-contingent, adaptive fashion. (Flave11, 1977, pp. $169-170\rangle$

Another position close to Flavell's is that of Maccoby (1969). She argues that: (1) children orient their sense organs more efficiently with increasing age and experience; (2) the ablitity to select a wanted stimulus out of a complex array increases with age; (3) selecting two portions (or more) of a complex stimulus is more difficult than selecting one and that this skill develops more slowly during childhood; (4) larger, stronger configurations, or strongly organized configurations handicap the selective perceptions of young children more than older children; and (5) with increasing age the child's perceptions are more dominated by organized search patterns that are related to sustained plans of the perceiver. 
There are numerous studies which support the notion of attention as a developmentally acquired skill. Eimas (1969) gave 270 children in grades $K$, 2, and 4 a simultaneous discrimination task with either 2,3 , or 4 relevant and redundant visual cues (color, form, size, presence or absence of borders). When four relevant cues were present, he found that the various grade levels could attend to 1,2 , or 3 cues respectively. Anderson and Leven (1976) found that within the two to four age range, younger children could not attend to television to the extent older children could. Pick and Frankel (1974) found that older children (sixth graders) could modify their at tention more flexibly than younger children (second graders) on tasks requiring flexible strategies of visual selection.

Thus, as Vinje (1978) concluded, there is a large attentional deficit that makes young children unreliable in traffic. This is particularly true for children under five. The use of salient clues to aid training these children may actually present an obstacle to attending to relevant aspects of the task.

\section{D. Spatial Relations}

Children's perception of space affects their safety in traffic in that it is important for them to know when they are within their field of view, where they are within a driver's field of view, and where other key elements (cars, roads, curbs, etc.) of the environment are located vis-a-vis their location. Children's capabilities regarding spatial orientation, like most of the other abilities discussed in this section, improve with age. Toddlers begin to master space in segments, rather than as a totality (Stone \& Church, 1968). A toddler may protest when mom makes a wrong turn or chooses an unfamiliar route on the way to grandma's house. Like "space-blind" adults, the child may know many specific locations but without understanding of an overall organization. As a preschooler matures, he or she moves slowly away from action-space to map-space. Maier (1936) had children aged three to seven learn all the routes of a maze and then tested the children for their grasp of the maze layout as a whole. Such an ability was not present in children younger than age five or six. Abstract space follows map space and involves the understanding of two or more dimensions. This ability is completely beyond the limits of preschoolers (Stone \& Church, 1968). 
One frequently cited impediment to children's ability to improve their spatial awareness is egocentrism. Furth and Wachs (1974) feel that an accurate visual perception of space does not take place until egocentrism declines. Piaget and Inhelder (1956) found that until about age seven children's spatial concepts were limited to their own visual perspective of the world; i.e., they could not grasp that others in their environment might have different viewpoints. In this case children viewing a mock landscape incorrectly assumed that a doll saw what they saw, regardless of the doll's location relative to their own.

Later evidence suggests that children's understanding of spatial relations begins earlier than age seven. Shantz and Watson (1970) feel that children first come to realize that objects and their arrangements look different from various spatial locations. Next these differences are formatted into specific object-subject relations, and finally the relations are organized into a total spatial framework. Their research to investigate the first step of this process demonstrated that three to five-year-olds will register surprise when their expectancies have been deceived (i.e., when something should appear different, but is not). Other evidence indicates that preschoolers make surprisingly good predictions of the location of objects when the child moves (Shantz \& Watson, 1971; 01son \& Baker, 1969).

Although children's spatial abilities are beginning to develop during the preschool years, it does not mean they can be trusted in the traffic environment. Vinje (1978) concludes that understanding of the front-behind dimension does not occur until age six. There is a variety of evidence to suggest that even the older preschool child cannot accurately predict changes in the front-behind dimension of visibility, a weakness which can be very fatal in a traffic situation (Nigil \& Fishbein, 1974; Hoy, 1974; Coie, Costanzo, \& Farnill, 1973; Minningerode \& Carey, 1974). Using a small scale mode1, Gunther and Limbourg (1976) also found that four and five-year-olds had much more difficulty than older children predicting the viewing possibilities of children and car drivers. In a study designed to determine whether walking to various viewpoints would improve visibility predictions for preschoolers, Vinje and Groeneveld (n.d.) found inconclusive results; the effects were very limited, and there was no generalization to other traffic situations. 


\section{E. Distance Perception}

Children's perception of distance is another skill that affects their ability to safely interact with traffic. In particular it is important in terms of judging how far away approaching vehicles are and how far they must go to get to the other side of the street, so that they can adequately judge the traffic gap before crossing. This ability also relates to the perception of velocity as well.

Adults use a variety of means to judge distances. Binocular differences, the slightly different retinal representation of objects, is one method. If either the object observed or the observer is moving, the optical expansion, or rate of size change, can be used. Rotary head movements can also provide cues, as can single eye or painters' cues, such as overlap, texture, density gradients and perspective diminution.

There is a great deal of evidence to suggest that even very young children are able to use some of these means to judge depth or distance. Gibson and Walk (1960) used a visual cliff to demonstrate that infants, aged six to fourteen months, who would eagerly approach their mother on the shallow side of the cliff, would refuse to cross the "deep" side, even with their mother's encouragement and coaxing. While this study does demonstrate that infants can discriminate depth by the time they can crawl, it is possible that this ability is acquired through experience.

More recent research involved testing infants as young as forty-four days in the same apparatus. Since premotor infants could not be expected to crawl, the researchers used heart rate differences to assess depth perception. As hypothesized, the infants placed on the deep side evidenced heart rate decreases, supporting the argument that human depth perception, much like that of kids, lambs, and chicks, is innate (Campos, Langer \& Krowitz, 1970). Bower has also provided results supporting the nativist viewpoint. Infants only a few days old will show an avoidance response to the distance of an approaching stimulus, not its retinal image (Bower, Broughton Moore, 1970). Six to eight-week-old infants would make such discriminations even with one eye, but when pictures rather than real objects were used, the infants could not 
discriminate on the basis of these painter cues (Bower, 1966). Newman and Newman (1978) review a host of other studies using different methodologies (fixation time, defensive reactions, and reaching) to examine sensitivities of newborns to distance cues. They conclude that by two months at the latest, there can be no doubt that the infant can use distance cues.

Although the ability to perceive depth may be innate, the importance of experience in maintaining early visual skills should not be underestimated. Wohlwill (1963) has demonstrated that older children and adults become familiar with the principles of perspective, and in cases where they think a visual illusion is present, they will overcompensate distance judgments. In contrast, younger children less familiar with these principles make more accurate distance judgments under those circumstances.

There are also distance perception illusions that reflect the effects of maturation or experience, either decreasing or increasing in magnitude, with age (Pollack, 1969). Illusions which decrease with age, like the Muller-Lyer illusions (two lines of equal length with arrows at each end pointing in or out), are rendered less susceptible, according to Pollack, because of increased foveal pigmentation. Sega 11, Campbe11, and Herskovits (1966) report a similar decrease with age for that illusion, but they argue that the susceptibility is strongest in carpentered groups, i.e., those who are exposed to physical settings with lots of right angles. Berry (1971) found that density of pigmentation and carpentering of the environment were inversely related. It thus appears that the adaptation of the human body to intense sunlight in certain environments is related to a style of building, a style of perceiving, and a pattern of preference for certain environmental configurations.

Pollack (1969) believes that illusions whose magnitudes increase with age, e.g., the Usnadze illusion (two concentric circles presented successively, the inner after the outer, with the inner tending to look smaller than it actually is), are related to intelligence, since it is necessary to use cognitive schema, memories, and anticipatory responses to create and maintain the illusion. 
Although even newborns have the ability to use distance cues, and although experience is important for honing these skills, adults do not always perceive distances accurately. Gibson, Bergman, and Purdy (1955) demonstrated that a) though perceived distance increases linearly with real distance, untrained aduits typically underestimate actual distance. Training did improve absolute judgments ( 57 to 395 yards), but it had little effect on relative (nearer-farther) judgments $(50,100,200$ yards). Ittelson (1951) demonstrated that one of the major cues adults use to assess distance is relative size, but he found a great deal of intersubject variability in this regard.

Earlier mention was made of Bower's (1966) research demonstrating the fact that infants show size constancy--the ability to perceive an object retaining its actual size independent of viewing distance. Ziegler and Leibowitz (1957) examined this ability at distances of 10 to 100 feet with seven to nine-year-olds and aduits. While adults did demonstrate size constancy, the children reached a limit at about 60 feet. The data were interpreted as supporting a developmental increase in size constancy. Vurpillot (1976) believes that both distance constancy and size constancy evolve with age and that as distances increase, the error of estimation does so too.

Zwahlen (1974) examined this topic in a traffic situation because of its safety relevance, because errors of distance judgment seemed to be involved in about 33 percent of the pedestrian accidents and because his previous work in distance perception demonstrated wide intersubject variances in this regard. He used twenty subjects (one-half six to thirteen years of age, one-half twenty to thirty years of age) to test this ability in the natural traffic environment and under simulated lab conditions as we11. The standard he used was a 1964 station wagon parked at 200 feet from the observer and the stimulus was a 1970 Maverick parked at 180 to 210 feet (using 5 foot intervals) from the subject. Adults and children did not differ in terms of their mean judgments, although the younger subjects had larger variances than the aduits. The laboratory results indicated that the children used less efficient head and eye movements; more time per movement and more time per fixation than adults. The basic strategies used, however, were the same for both groups, as they had similar ratios of head movements to foveal fixations. 
Thus, it seems that children have the ability to use a variety of distance cues very early in $11 \mathrm{fe}$, but that does not mean that they can use this ability in the typical traffic situation. While there is no data on preschoolers, it seems that occasionally adults, and even more of ten older children, cannot depend upon their own estimation of distances in the traffic situation. Younger children can be expected to perform less accurately in those situations.

\section{F. Perception of Velocity}

Children's perception of velocity requires the use of vision, distance judgments, audition, spatial perception, and other skilis, including the ability to process this information, and therefore it is not surprising that this capability also improves with age. Piaget (1970) believes that the understanding of movement and speed is closely related to the child's concept of time. A long elaboration process beginning in the sensorimotor period and working through to the operational period is required, during which time the child uses six distinguishable operational systems. The first four stages depend only upon qualitative logic; only the last two stages require quantitative logic, and then only the last step truly involves a thorough understanding of the concepts and includes the use of or ability to use measurement with repeatable units.

Vinje (1978), citing a variety of studies (most of which we were unable to obtain) which generally support Piaget's position, concludes that while children around eight years of age can compare speeds that are presented simultaneously or in rapid succession, they are affected by the order in which objects move. They do not have a time concept of speed; passing more objects means going faster, regardless of elapsed time. This passing effect is stronger when children cannot actually see the movements (Boyle \& Gilholly, 1972; Oppenheimer, 1975; Gunther \& Limbourg, 1976; Fraisse \& Vautrey, 1952; Uruno \& Yoshibe, n.d.l. 
Vinje (1978) also reports on research that indicates young children do not have the knowledge to determine the speeds of various vehicles; only $60 \%$ of four to seven-year-old children possessed such knowledge (Hartwig, 1968; Gorges, 1973). He does not state what types of vehicles were included or the type of responses solicited from the child.

Environmental theorists include Brown (1931) who stated that perceived velocity is a direct function of perceived distance and time. This law is limited in its generality however, as more recent research found that velocity is finest when the spatial cue alone is present, intermediate when the temporal cue only is present, and poorest when neither stimulus relevant cue is present (Mandriota, Mintz, \& Notterman, 1962). J. J. Gibson (1968b), another environmental theorist, feels that velocity judgments need not be based on only visual information but that they can arise from auditory, kinesthetic, vestibular, proprioceptive, and other data as well.

Avery and Day (1971) provide empirical support for this viewpoint. They used adult subjects to examine the horizontal-vertical (HV) velocity illusion, i.e., an object moving vertically apparently moves faster than one moving horizontally at the same physical speed. They found that judgments of contour, length, and moving point velocity are mostly independent, and they conclude that the mechanism and processes mediating these judgments are mostly independent of each other. However, since length judgments were affected by a moving point, they feel that there is some interaction between these two processes.

Salvatore (1974), also providing support for Gibson's position, found that auditory cues and vehicle size affect velocity judgments and that these cues may override visual velocity cues. He asked 40 children, two of each sex at each age level from five to fourteen years of age, to make slow, medium, or fast judgments regarding vehicle velocity from both 500 -foot and 250 -foot observation distances. Correct judgments of slow and medium speeds increased with age, while correct judgments of fast were inversely related to age. Younger children, probably responding to parental road safety warnings, overused the "fast" clarification; in contrast, older boys underused that category. Older girls tended to overestimate speeds, but there were no sex 
differences for the younger children. Observation distances also influenced velocity judgment. As expected, discrimination became more difficult as the observer-vehicle distance increased. These findings have been replicated and extended to show that estimations of vehicle arrival time improve through at least age ten (Hoffman, Payne, \& Prescott, 1978).

Physiological theorists generally use electrophysiological research methods to seek evidence of motion-sensitive units in the visual system. Levick (1965) found evidence of cells receptive to slow stimulus movement at or before the ganglion cell layer of the retina in the rabbit. Hubel and Wiesel (1965b) found three different types of cells in the visual areas of cats which respond best to particular stimuli such as slits, edges, or bars, and particular velocities, in one or both directions. Also working with rabbits, Barlow, Hill, and Levick (1964) found that two synaptic layers of ganglion cells abstract direction, speed of motion, and localized diming and brightness from the retinal image.

Ethical concerns have prevented electrophysiological studies of movement sensitive units in the human visual system, but there is psychophysical evidence for the existence of such units. Carison (1962), in a study with 19 college juniors, found that prolonged motion stimulation in one direction diminished the sensitivity to movement in that direction. Little or no aftereffect occurred when adaptation and test motion were in opposite directions however, thus supporting the existence of retinal units which are differentially sensitive to direction. Sekuler and Ganz (1963) also found similar results.

There is also psychophysiological research which indicates that the human visual system has several velocity-sensitive mechanisms, each responsive to a specific range of contour velocities (Pantle \& Sekuler, 1968; Sekuler \& Pantle, 1967).

As mentioned earlier, these studies have not examined developmental aspects such as motion detection. However, Lovell, Kellett, and Moorhouse (1962) used a Piagetian task with 60 primary and 40 subnormal children to examine the development of logic in velocity perception. They found that most 
of the five- and six-year-olds could discriminate between faster and slower velocities with trains which start simultaneously and travel equal distances but vary in time of arrival. When one train traveled a longer, more circuitous route but started and stopped simultaneously with the other train, it took until age nine for $75 \%$ of the children to correctly select (and justify their choice logically) the faster of the two trains. Nine-year-olds could correctiy judge that when trains are traveling at the same speed, the longer journey will take more time.

Thus, it appears that preschoolers, if they have any capability to judge velocities accurately, can do so only in the simplest cases. It is unlikely that the traffic environment would be kind enough to offer them only simple cases however. It is also evident that there is little direct evidence dealing with this ability during the preschool age, so further research will be needed to isolate the reasons that preschoolers have problems in this regard (cognitive, physiological, experimental, etc.) and whether training of some type might be of benefit.

\section{G. Causality}

Children's understanding of causality is another skill which develops with age. Sandels (1970) reports that young children belleve that adults will always be kind to them and that drivers of cars will be able to stop instantly if a child is threatened. There is supporting evidence that children as old as eight years of age fail to perceive cause-and-effect operations in interpersonal relations (Bobroff, 1960). They seem to feel that powers exist which can solve all problems and make everyone happy. Husband (1973) also reports that children expect nothing bad to happen to them in the traffic situation or that by simple means (i.e., crossing fingers) they can protect themselves.

While understanding of causality in terms of social relations may develop slowly, physical causality develops in infancy. A four-month-old can easily master a task involving striking a switch to turn on a light several feet away, even though an eight-month-old will have trouble with this task (CRM, 1971). The older infant has begun to understand causality, at least for 
proximal events. His imperfect knowledge about spatially separated cause-effect causes him problems. The four-month-old takes it in stride, as if it were magic, with no explanation required.

Piaget (1952) feels that children's first perception of causal relationships is animistic; i.e., things move, act, and react in terms of their own built-in thoughts, feelings, purposes, and volition. The care children give to certain toys and stuffed animals is evidence that they perceive things animistically, as is the anger and displeasure they can show to the same objects when the objects do not follow the child's wishes. Animism is succeeded by artificialism, the assumption that events happen because some agent or force wills it for purposes of its own. of ten children will ask, "Who made it snow," or "Why did they make the leaves fall off the tree?" For Piaget, true rational causality does not appear until age seven or eight (Piaget, 1930). Thus these limitations place the preschool child at risk in terms of still one more ability in relation to the traffic environment.

\section{H. Short-Term Memory}

Memory is another capability that affects children's safety as pedestrians. Short-term memory, which affects the child's ability to remember information about the position, speed, direction, etc. of traffic for any particular crossing activity, will be discussed in this section.

There is evidence indicating that short-term memory develops during childhood. Three-year-olds can recall only a series of three repeated numbers, while seven-year-olds can recall five (Hetherington \& Parke, 1979). A tachistoscopic presentation to adults and five-year-olds indicates that adult's short-term memory was four items and the children's was less than two items (Haith, Morrison, Shingold \& Mindes, 1970). Avery (1974) concludes that the short-term memory of children is considerably poorer than that of adults and speculates that lower performance is due to the fact that the short-term memory of children cannot learn and store as much information as an adult's short-term memory. He believes their rate of forgetting may differ very little from aduits and cites support from studies which indicate that from kindergarten on, children use the same memory search processes adults use 
(Belmont \&utterfield, 1969; Hoving, Mor in \& Konick, 1970). As will be noted be low under long-term memory, more recent research contradicts this latter conclusion (Kobasigawa, 1974). Belmont and Butterfield (1969) have also concluded that different age children have the same rates of forgetting from short-term memory, and they suggested that the impaired memory performance of young children is not a function of their capacity to remember, but of their lack of expertise in determining the relevant things to remember and their lack of use of encoding mnemonic devices. Even children in grades $K$ and 2 are not able to process information for storage in short-term memory as quickly as older children and adults (Cermack, Sagotsky, \& Moshier, 1972; Gummerman \& Gray, 19721.

Vinje (1978) has concluded that there is both qualitative and quantitative development in children's short-term memory. The codability of information and its resemblance to the structures the child has at his disposal affect the amount and manner of storage. Because position and orientation of objects are particularly difficult for young children to remember, Vinje feels that preschoolers will have difficulty in remembering the position and driving direction of oncoming traffic, particularly when they are on an unfamiliar street. However, the accident data indicate that most children are hurt close to home, implying that some other compensatory mechanism operates in that situation, or that the mechanism fails to operate in familiar surroundings. Vinje also concludes that children under age seven have little likelihood of interpreting information such as speed and distance, so the limitations of short-term memory for these children are somewhat irrelevant given those more serious limitations. Other capabilities aside, the limited abilities and experiences of preschoolers still place them at risk in traffic in terms of short-term memory. This implies that young children may have more than they can safely handle crossing even the simplest traffic lanes alone.

\section{I. Reaction Time}

In the traffic situation a child frequently has to process incoming sensory information, usually visual or auditory and then react to it in some manner. The amount of time for this process to occur is referred to as reaction time. Laboratory studies have provided a great deal of evidence 
indicating that both auditory and visual reaction time decrease with advancing age.

Elliott (1964) compared adults and kindergarten children in an auditory reaction task and concluded that the poorer performance of the children was "due primarily to the fact that the children were far less able than the adults to maintain an undivided and persevering attention to their task" ( $p$. 30). In a later study (Elliott, 1970), he rules out physiological differences to account for young children's deficits in this area since children (six-year-olds) have the same latency of initial wave-evoked potential visual signals as adults (sixteen-year-olds), and they have shorter nerve pathways but with the same conduction velocities as adults. He indicates that preparatory set, the readiness to respond to a signal, is the central factor responsible for children's deficits. His research with simple auditory reaction time tasks for five to thirteen-year-olds and adults reinforced his earlier conclusion that children were not attending to the tasks as closely as adults. He also feels that attempts to train children to do well in such tasks are necessary to really determine the magnitude of adult-child response time differences.

Pick, Christy, and Frankel (1972) used a visual reaction time task with second and sixth graders. They attributed the shorter reaction times of the older children to developmental improvements in selective attention. Blake (1974) examined the visual processing speed of four-and eight-year-olds and adults. Eight-year-olds approached speeds and limits of adults and seemed to use the same strategy, whereas four-year-olds had much lower capabilities than the two older groups and a completely different, less effective, processing strategy. Liss and Haith (1970) found that tasks which involved visual search slowed the processing speed of four-and five-year-olds but not older children or adults.

Echert and Eichorn (1977) examined auditory reaction times in four and a half through sixteen-year-old children. They found a consistent improvement in mean reaction time from 4.5 to 16 -years of age. The largest improvements occurred during the four and a half to five and $a$ half and five and a half to six and a half age ranges. They conclude that the improvements they found 
with age and experience were the results of a major maturational component and a very minor learning component.

Cratty (1979) advances two other theories besides the attentional deficit explanation that might explain the decrease in reaction time as a function of maturity. One of these, proposed by Luria (1932), holds that the general excitation produced by the stimulus spreads not only to the motor system, but also to other parts of the brain, causing responses which interfere with the appropriate response. As children mature, they build up a functional barrier to such diffusion. On the other hand, Surwillo (1971) believes that the faster reaction times of older children are produced by neurological maturation and associated information processing differences rather than attentional or diffusion differences. He based this conclusion on findings indicating changes in brain wave functions paralleling reaction time improvements and the fact that artifically speeding up or slowing down those brain waves altered reaction time. He also concludes, as do Echert and Eichorn (1977), that improvements are most rapid in younger aged children.

Thus, there is a variety of evidence to indicate the deficits young children have in this area, and there are several possible explanations for these deficits. While findings are based primarily upon laboratory results, there is no reason to suspect that preschoolers would show much improvement in a natural setting. This is especially the case since this capability is highly dependent upon attentional and motor skills, capabilities that are also very limited for this age range, and since activities which require a visual search, while the traffic setting does, seem to exacerbate the deficits in young children.

\section{J. Motor Behavior}

The ability to walk and otherwise interact with the environment is clearly a capability most pedestrians develop as preschoolers. The developments that can be expected from various age ranges have been described by Gesel1 (1940). As a preschooler approaches his/her third birthday, walking becomes automatic. Balance has improved, and the child may frequently try stunts beyond his/her ability. He/she can run and gallop, jump from a height of 18 
inches and walk a straight 10 foot path, one inch wide, stepping off only two or three times. Preschoolers at this age can then walk and attend to some other tasks, but there is no indication that they can attend to a complicated tasks such as watching for traffic yet.

Between ages three and four, the child gains the ability to walk with uniform length, width, and speed of step. He or she can balance and walk backward with ease, as well as run easily and smoothly. Cratty (1979) points out that until about four and a half or five-years of age this running ability is not matched by an ability to start efficiently or stop quickly.

Between ages four and five, the child improves steadiness and gait. Better coordination of movement of all body parts is evident. Running can be done smoothly at different speeds, and sharp corners can now be negotiated. Starts and stops can now be accomplished quickly and easily except under distracting or emotional circumstances. Between five and six the preschooler improves the abilities he or she already possesses. Strength, grace, and economy of movement all improve.

Gunther and Limbourg (1976) had four and five-year-olds and six to nine-year-olds turn a wheel to make a toy car drive a track. The younger group could not stop their movements within a second after the presentation of an auditory or visual sign, but the older group could. As vinje (1978) points out, this is a quite different movement from walking or running. He also points out that the generalization can be made that preschoolers cannot be expected to come to a sudden stop. Although it does not follow directly from the Gunther and Limbourg results, the limitation may be true based on Gesell (1970), Cratty (1979), and other research on reaction time and motor behavior.

Thus, the preschool period encompasses a wide range of motoric abilities on the part of these children. The changes occur fairly rapidly and are one of several reasons the use of separate educational materials for relatively narrow age ranges seems appropriate. 


\section{K. Impulsivity}

Sandels (1975) has noted that young children have difficulty surpressing sudden impulses and are therefore likely to run into the road without warning. A glimpse of an object, a barking dog, an impromptu game of tag, or any number of similar circumstances, real or imagined, can trigger such impulsive behavior. The evidence is clear that this behavior is a major problem, since dart-out type accidents are by far the most prevalent accident type young children are involved in, as has been noted earlier.

There is no directly relevant research addressing the developmental aspects of this behavior that we have been able to locate. However, there is a great deal of evidence that as children mature they are better able to inhibit immediate impulses and respond in a more cognitively or iented manner. Such research typically examines the reflective-impulsive disposition of the child. Children classified as reflective tend to gather more information and respond to a given situation more slowly and thoughtfully than children classified as impulsive.

White (1965) has reviewed this issue and concluded that young children are poor at response inhibition. They can follow commands for action, but not for restraints of action. Young children also have difficulty restraining their first-available response in favor of a later response. This situation does improve with age, however. White's model indicates that the crucial stage is during the five to seven year period, when the child has matured and gained some experience as we 11, that the associative function can be inhibited in favor of a higher level cognitive function.

Kogan (1976) and Messer (1976) both agree that there are developmental aspects of reflection-impulsivity. Further, these effects may vary with sex and SES. Kogan (1976) provides evidence that this dimension does relate to motor inhibition, a necessary link if these laboratory data are to have any relevance or meaning for the traffic task.

The remaining issue concerns training; can the disposition to impulsivity be modified? Kagen and Kogan (1970) and Messer (1976) review a variety of 
methods (forced delay, reinforcement for increased response time or decreased error, modeling, and direct use of scanning strategies) which attempted to accomplish this goal. Both reviews conclude that it is possible to modify this disposition, although the studies reported used school-aged children, not preschoolers. Some teaching strategies were more effective than others, notably the combination of teaching scanning strategies with appropriate training materials and having the child verbalize the strategy aloud.

Thus, children's impulsivity is a problem. For the age range of interest, the safest course of action seems to be to keep the child away from situations where impulsivity will threaten his or her safety.

\section{L. Risk Perception}

Vinje (1978) reviews a number of studies on this topic to determine whether young children behave unsafely in traffic because they lack the appropriate capabilities or if perhaps they simply do not see the risks involved in their actions. Gunther and Limbourg (1976) examined the pulse rates of children who played with a doll crossing a street with toy cars present. Ocio (1973) used a similar situation: only the doll was a car passenger. Neither study revealed any pulse rate changes, but there was no evidence that such measures were related to actual risk perception. Galvanic skin responses were affected by looking at pictures of dangerous traffic situations. Nine and ten-year-olds apparently exhibited signs of anticipation for these risky situations, unlike five to eight-year-olds who gave no such signs (Heinrich \& Langosh, 1976; Finlayson, 1972).

In the U.S., Martin and Heimstra (1973) examined the hazard perception of almost 1,500 children (grades 1,3, and 5) from a variety of backgrounds. The children were given the Perception of Hazard Test, a series of 10 photographs that they were asked to rate in terms of risk using a five-point scale. Pictures used included a street scene with a model unprotected in the middle of the street directly in the path of an approaching car. Age, sex of the child, sex of the model, geographic and socioeconomic variables were all found to influence the perception of risk. In terms of the traffic picture, there were no respondent sex differences, but the sex of the model made a great 
difference. When the model was female, risk was generally underestimated relative to the adult estimates, especialiy for the youngest respondents. The opposite was true for male models; the risk was overestimated, particularly by the older children.

Unfortunately these studies do not shed a great deal of light on the ability of preschoolers to perceive risk. It may be inferred that a variety of influences will affect their choice, but until further work with this age range is undertaken, their ability in this regard can only be speculated.

\section{EDUCATIONAL CAPABILITIES OF PRESCHOOLERS}

\section{A. Long-Term Memory}

While there has been research on the long-term effects of traffic instruction on children discussed elsewhere in this report (J. A. Rothengatter, 1977, 1978; T. Rothengatter, 1976), this research is not really memory research. Vinje (1978) feels that real-memory work is distinct from the practical contents of traffic instruction and that generalization from that work would not be in order. However, for the purposes of this study, and for the age range of interest, it seems important to examine this area further to study the evidence on the limitations of long-term memory in preschoolers, and see what ablities they have to remember instructions. There is evidence for a clear developmental trend in long-term memory (Hetherington \& Parke, 1979). Brown (1975) has made a distinction between memory as knowing, memory as knowing how to know, and memory as knowing about knowing, and these processes provide a means to examine the developmental differences in memory. The knowledge children have influences how they store and retrieve new information. Children of all ages use an organized and constructed manner to store information rather than storing it in a random fashion, but the process varies with age. One such way is the increasing use of categories and verbal labels as children grow older (Flavell \& Hellman, 1977). Children, like adults, may also remember things in a way which seems logically correct to them, and they may later have trouble distinguishing their logical elaboration from the original event (Bransford \& Franks, 1971; Paris \& Lindauer, 1977). 
Memory as knowing how to know can also account for developmental differences. 0lder children and adults use intentional strategies to improve memory more often and/or more effectively than younger children. Flavell (1970) examined the use of rehearsal in children from grades $K$ through 5 , and found that children whose lip movements indicated spontaneous verbal rehearsal remembered better than those who did not. Further, use of spontaneous rehearsal increased from $10 \%$ among kindergarteners to $85 \%$ of fifth graders. These results were interpreted as a production deficiency for younger children, not a mediational deficiency, since when the experimenter supplied the label or instructed the younger children to label, their memory improved greatly. The use of images has also been demonstrated to aid young children, grades $K$ through 3 , in remembering (Wolff \& Levin, 1972). There is also evidence that the organizational basis for memory, the pattern of clustering used to store information, changes with age, with younger children showing more evidence of a production-deficiency in clustering than older children even within the two to five age range (Rossi \& Wittrock, 1971). In terms of retrieving information from memory, there is evidence that third and sixth graders use plans and strategies more effectively than first graders (Kobasigawa, 1974).

Knowing about knowing also develops with age, as older children become increasingly aware of their own memory ability, of what they find easy or difficult to remember, and they learn when to use special strategies to aid memory. Fifth graders are more likely than first graders to study harder for a difficult test than an easier test, to use appropriate memory aids, and to predict when they have studied enough to retain the material (Flavell \& Wellman, 1977; Markman, 19731.

Thus, in terms of long-term memory, there does appear to be a developmental trend, and the development continues well past age five, so preschoolers are nowhere near their peak memory capacity. The main exception to the above was research conducted by Nelson (1971) which found no long-term forgetting or short-term (immediate retention) differences in children from age seven to thirteen, although memory for reconstruction of six or nine card matrices did improve with age. While this is beyond the age range of interest, it is still worth noting that even for those ages, the absence of a developmental trend appears to be an atypical finding. 
Yeaton and Bailey (1978) have demonstrated that elementary school children can retain the effects of traffic safety instruction for at least 12 months. They used an instructional package implemented on the street corner to teach six crossing skills to children in grades $\mathrm{K}-3$. The immediate impact of the training was significant, and the skills were maintained, or easily recovered by remedial training, over a one-year period. Further research will be needed to determine the abilities of preschoolers to retain traffic safety instruction for any length of time.

\section{B. Understanding Rules}

A child's ability to understand and obey rules can also affect his or her safety in traffic situations. There is considerable evidence to indicate that this ability is a cognitively based skill which improves with age. Piaget (1972) feels that unt $\$ 1$ age seven, children do not demonstrate an understanding of games with rules. Sutton-Smith (1971) describes a number of games played by young children (Ring Around the Rosie, Farmer in the Dell, etc.) which are almost a ritualized form of symbolic play. They do involve rules, defining parts for each participant and the sequence of action, but there is little or no true understanding of rules. Eiferman (1971) examined the school recess play of about 14,000 Israeli children between the ages of six and fourteen. She found a peak of interest in rule-governed games from ages six through ten, reflecting the rule awareness of the concrete operational child. However, unlike Piaget's belief that rule-governed games remain as a central form of play until adulthood, she found a marked decline in interest that set in as early as second grade in some schools.

Bobroff (1960) acknowledges that children can follow game rules, when they are in the six-to eight-year-old range, but until they are ten or so, they are not fully cognizant of the role of rules in regulating the behavior of interacting persons. While younger children may demonstrate inflexible adherence to rules, by age ten, children generally master the details of the game rules and follow them correctly.

Merchant and Rebelsky (1972) found that five to seven-year-olds who participated in making up rules were more flexible in changing the rules than 
control children who were not part of the rulemaking. Within this age range there were no grade $K$-grade 1 differences. It was concluded that the experience of rulemaking showed those children that they could make rules, and that rules can be made by mutual agreement for cooperative purposes. How such children or even younger ones react to the road-crossing where "one is basically laying down inviolable rules for children to follow" (Avery, 1974, p. 19) remains to be seen. The fact that younger children show less flexibility (i.e., rule-bending) than older children may in fact offer them more protection in the traffic situation, although their inability to adapt to changing environments may simultaneously offer them less protection.

Gagne (1970) believes that the learning of rules occurs late in the developmental hierarchy of learning. To learn a rule, the child must be able to chain together two or more concepts. Acquisition of this ability is dependent upon the mastery of earlier types of learning: stimulus learning, stimulus response learning, chaining, verbal association, multiple discriminations, and concept learning. He speaks of developmental readiness, but does not provide specific ages for these types of learning. Concepts such as rules, taking turns, fairness, etc. are still elusive for preschoolers (Newman \& Newman, 1978). Children are usually more intrigued with the rhyming and cadence of the rules and regulations they recite, rather than comprehension of the material. Pease and Preston (1967) found that children who were able to recite a "Kerb drill" (i.e., When you cross the road by day or night/Beware of the dangers that loom in sight./Look to the left, and look to the right/Then you'll never get run over) were not able to implement this lesson. Children were found to regard left and right as parts of the environment rather than directions in relation to themselves. Further, the recitation of the drill was more important to the children than implementing its lesson. The authors conclude: "It is felt that the way in which the child undertakes the drill in the experimental situation lends support to the interpretation that the kèrb drill is seen as merely a safety rite to be gone through crossing the road, in that the child's movements appeared anything but natural, and the eyes were directed right and left without apparently focusing on what was there. The way in which many of the children walked across the road during the experiment was for the most part extraordinarily stylized" ( $p$. 
312). However, Rothengatter (1977) provides a methodological criticism of this study and questions the conclusions presented, despite their face validity.

Thus, the understanding of rules is an ability beyond the reach of most preschoolers. Any rules used for this age range must be very simple, and little faith should be placed in their likelihood of being followed. Primary emphasis at this age should be placed on other concepts and abilities that emerge before the understanding of rules.

\section{C. Concept of Left and Right}

Children's ability to understand the concept of left and right also develops slowly and later than the before-behind relationships discussed earlier under spatial relations (Piaget \& Inhelder, 1956). Vinje feels that children begin to understand this concept in terms of their own body by age five or six, but that until age nine or so, it will not be developed enough for use in traffic. Even then, as Sandels (1975) points out, a significant numter of nine-year-old children cannot use this ability to watch for traffic. The deficit young children display regarding this concept goes beyond not understanding the terms. Five-year-olds who saw a film of a girl facing them perform a perfect road crossing, became confused about which direction to look first, even though they had done so correctly before watching the film of the girl model (Pease \& Preston, 1967). Thus, this concept appears to have minimal instructional utility for preschoolers.

\section{D. Communication}

A child's ability to communicate affects his or her ability to understand instructions regarding traffic safety, to comprehend commands and traffic signs and signals, and to deal with both adults and peers in the traffic situation. There is convincing evidence from a varlety of sources that children's communication abilities improve during childhood. The discussion here will be limited to post-infancy periods, even though there is extensive evidence that infants can and do communicate effectively well before they learn to talk. 
Reference is one of the basic functions of language; it is easy to conceptualize, and thus, it has received a great deal of attention recentiy. Much of this interest has been prompted by the concern voiced by Glucksberg and his colleagues that the increased attention devoted to linguistic development has not been paralleled by concern for the processes by which children learn to use language to communicate (Glucksberg. Krauss, \& Higgins, 19751.

Wellman and Lempers (1977) examined the referential communications of ten two-year-olds in a nursery school setting. They videotaped and scored the communications in which one child wanted to point out, show, or display a particular referent (object) to another child. The results indicated that two-year-olds were surprisingly sophisticated in their comunication abilities. The speaker usually addressed the listener when they were together and even more of ten when the listener was uninvolved. Speakers and listeners were almost always within sight of each other and physically close, and of ten in direct eye contact with each other when the communication began. Moreover, speakers and listeners were almost always near the referent. Most of the communications received an adequate listener response. Fur ther, these children adjusted their communications appropriately (more communication in difficult situations, less in simple situations). The speakers were also very adept at using the feedback the listener provided, of ten repeating and clarifying messages when necessary but rarely when it would be redundant.

Shatz and Gelman (1973) examined the referential conversations of four-year-olds who were introducing a new boy to adult partners and two-year-old partners. They found that the four-year-olds switched their speech pattern to accommodate the different audience: shorter utterances, and more attention-getting and attention-holding words with two-year-olds, the opposite with adults. The complexity of the grammatical construction was appropriately adjusted as well, and adults received communications which were more polite and cautious than those for the two-year-olds.

Research with four- and five-year old speaker-listener pairs who were visually separated provides a somewhat different picture regarding children's capabilities. When the referents were novel graphic forms, the pairs could 
not communicate adequately (i.e., the listener could not select the appropriate target described by the speaker). When pictures of familiar objects were used, performance improved (Glucksberg, Krauss \& Weisberg, 1966). A later study with children from grades $K, 1,3$ and 5 indicated there were no initial performance differences, but that practice rapidiy improved performance for the older children (Krauss \& Glucksberg, 1969). Whitehurst (1976) has also demonstrated a developmental improvement for speakers within this age range; Cosgrove and Patterson (1977) found a similar developmental trend for listeners of this age. Seven-year-olds are also somewhat better at utflizing listener feedback than four-year-olds, although the type of feedback offered seems to be a more dominant factor than age (Peterson, Danner, \& Flave11, 1977). Explicit verbal messages were effective for both ages; facial expressions were ineffective for both ages. Only the ability to respond to relatively inexplicit verbal expressions of noncomprehension improved with age.

A variety of efforts have been undertaken to train or improve children's referential communication abilities. Researchers conducting early studies in this area assumed that problems in these referential situations were primarily the result of egocentrism, believing Piaget's (1926) argument that children under seven or so years of age will be unable to accommodate the perspective of the listener. Many of the early studies used hours of practice in mostly unsuccessful attempts to improve children's communicative skills (Fry, 1966, 1969; Shantz \& Wilson; 197two, Oickson, 1974). Later studies which assumed the problem was something other than eogcentrism met with considerable success. A brief 30 -second plan offered to listeners in grades $K$, two, and 4 , emphasizing the importance of asking for information when appropriate, significantly improved their communicative performance (Cosgrove \& Patterson, 1977). A later study found that modeling proper strategies was also effective, that the effects of plans and modeling could generalize to different stimuli, and that the effects persisted for up to two weeks or more (Cosgove \& Patterson; 1978). An analys is of the $600+$ questions asked by isteners in the former study revealed only three that could be classified as egocentric. Thus, these studies and those discussed earlier, demonstrating the abilities of even two- and four-year-olds to adapt communications appropriately, fail to support the Piagetian notion that egocentrism is the prime contributor to children's communication failures. In fact, a recent 
study explored the possibility that such failures might be due to either comparison deficits (a failure to compare referents to information provided by the speaker) or to action deficits (not knowing what to do if the comparison process revealed that a message was inadequate). The results were clearly consistent with the action-deficit hypothesis, indicating that young children of ten realize when they have a communication problem, but they simply are not sure how to solve the problem (Patterson, Massad, Cosgrove, 1978).

These studies demonstrate the importance of encouraging children to ask questions when they do not understand a communication. Parents and teachers of ten assume that if a message is unclear, the child will automatically ask for clarification, but this is simply not the case. However, with very simple techniques, children can be trained to do this. There are also a number of nonverbal cues which preschoolers exhibit when they fail to comprehend a message (Patterson, Cosgrove, \& 0'Brien, 1980). Parents and teachers can be trained to recognize these signals (increased hand movements, longer response times, increased eye contact, and for $k-4$ children, but not preschoolers, increased body movement) and adjust their instructions accordingly.

\section{E. Vocabulary}

Another concern that parents and the educators of young children need to remain cognizant of is the limited vocabulary of preschoolers. A comprehensive investigation of children's vocabulary was conducted by Smith (1926). She used pictures to elicit words from preschoolers, and also gave credit for knowledge if the child answered a question which implied understanding of a key word. While the data are probably an underestimate for today's children, they still provide a useful gauge of a child's vocabulary at a given age and an estimate of the rapid growth of vocabulary. She found that two-year olds have a vocabulary of almost 300 words, mainly nouns. By two-and-a-half the child is rapidly increasing his or her vocabulary on a daily basis to about 450 words. At age three the child has a 900-word vocabulary. This trend continues through childhood. By age six it is over 2,500 words (Smith, 1926). 
The fact that children have words in their vocabulary does not mean they can comprehend their meaning. Asch and Nerlove (1960) found that preschoolers had great difficulty understanding that words can have psychological as well as physical meaning (e.g., sweet, cold). They could apply such adjectives to only physical objects. When they begin to apply the psychological properties of these terms, they do not realize the relationship to the physical properties. Comprehension of both contexts did not develop until past age twelve. Schreiber and Lukin (1978) found that children (3.5 to 8 years of age / could identify situations as "safe" or "dangerous," but without understanding the factors that made the situation safe or unsafe.

The comprehension issue is important in terms of road safety materials too. Cattell and Lewis (1975) examined the understanding of traffic-related words in children aged five to eleven. They found a variety of age differences and compiled a list of several hundred words and their understanding rating by each age group. This list provides a number of important findings, e.g., "behind" is understood by $96 \%$ of the five-year-olds, and "before" by $95 \%$, but "ahead" is only understood by $39 \%$ of these children. Sargent and Sheppard (1974) spent a great deal of effort to test children's understanding of words and phrases for use in the Green Cross Code, because there is wide variance in this regard and because it is so important to maximize understanding. Firth (1975) analyzed road safety descriptions given by 400 children aged six to twelve and found that very few children mentioned road safety directly or adequately, and that there were significant age differences regarding the frequency of use of words and phrases associated with roads and road safety, including mention of the "Green Cross Code", which was the most commonly used term by all ages. The term "kerb drill", on the other hand, was rarely used, and there were no significant age differences for that term. Sandels $(1970,1975)$ reports similar findings with Swedish children. There is also considerable variability among five- to eight-year-old Japanese children in their understanding of traffic terms (Saito, Nakamura, Hoshi, \& Hibi, 1978). 


\section{F. Traffic Signs and Signals}

Children's knowledge and comprehension of traffic signs and signals is a1so poor. Sub-Group II (OECD, 1978) reports on several studies, apparently with German children, dealing with this topic. Hartwig (1969) found that a third of over 100 children aged four to seven could not identify even one of four road signs shown to them. Michalik (n.d.) reports five- to six-year-olds have great difficulty understanding which side of the traffic light in an intersection applies to them. Richard (1975) reports that while about $50 \%$ of six- to eight-year-olds regulate their behavior primarily according to traffic signals, $30 \%$ believed that they should use the behavior of other people as a mode1. Sandels (1975) reports on misconceptions preschoolers have about traffic signs (e.g., pedestrian crossing signs referring to adults only, or school/playground signs meaning run across the road to avoid being run over). She summarizes childrens' abilities in this regard:

The studies of signs showed an astonishing lack of knowledge of traffic signs both on the part of playschool and primary school children. Both the lack of interest in the signs and the misinterpretations show the extent to which these small children are immature road users and how little ability they possess in this situation. (p. 100)

It is unclear from research conducted to date whether the limitations preschoolers have are due to primarily poor instruction, or to a natural limitation on their ability to understand such abstract problems, or to a combination of these factors. Further research will also be needed to identify the ages at which children can begin to receive useful instructions about signs and traffic signals.

\section{G. Logical Operation}

Parents and educators must also be made aware of the limited ability preschoolers have to understand terms referring to logical operations (negation, connectives, either - or, etc.). This ability also appears to develop during early childhood, but slowly. Feldman (1972) conducted research on several hundred three- to seven-year-olds to examine their understanding of negation. The children were asked to hand the experimenter "all the blocks 
that are not red," or "all the non-red blocks," or vartous other specifications. The findings indicated that understanding of negation develops slowly with age and experience and that it involves cognitive operations; it is not just a problem of semantics, since there were no sex differences despite the greater language superiority of girls. Further, there was little overall difference between familiar and unfamiliar idioms, although the "except - but" idioms ("give me everything but $x$ ") were easier than the "not" idiom. She concludes that logical connectives are acquired in hierarchical order ( $x$ and $y, x$, either $x$ or $y$ (not both), $x$ or $y$ (nonexclusive), not $x$ ). Thus, in terms of traffic safety instruction, negation, if it is to be understood at all by young children, should only be of the simplest kind. The message "Don't cross when cars are near" can be simplified to "Cross when no cars are near" or better still to "Cross when cars are a long way away." Research on other logical operations has similarly demonstrated the limitations young children have understanding such terms. Snow and Rabinovitch (1969) found that within the five- to thirteen-year range, disjunctive concepts (e.g., large or white cards) were more difficult to understand than conjunctive concepts (e.g., large (and) white cards). The difference in difficulty was constant over all age groups. Neimark and Slotnick (1970) examined the understanding of logical connectives in children (grades 3-9) and aduits, and found that most third graders understood inclusion (A) or exclusion (not $A$ ), but not intersection ( $A$ and $B$ ) or union (A or B). Shapiro and O'Brien (1970) examined the ability of six- to thirteen-year-olds to recognize and test for logical necessity. Test items included questions such as:

If this is Room 9, then it is fourth grade.

This is Room 9.

Is it fourth grade? (Yes, no) and.

If this is Room 9, then it is fourth grade.

This is not Room 9.

Is it fourth grade? (yes, no, not enough clues) 
The authors conclude that, while there is evidence of growth over this age range, "hypothetical deductive thinking-at least that which is consistent with mathematical logic-cannot at all be taken for granted in students of elementary-school age" (p. 829).

Thus, it appears that preschoolers' abilities in this regard will be even poorer than those for elementary school children. Materials designed for preschoolers must, therefore, not only attend carefully to the vocabulary, the traffic terms, and the logical connectives used, but they must also draw every conclusion for the child, since it appears that they cannot be relied upon to draw even relatively "simple" conclusions on their own.

\section{H. Crossing Judgment}

There is little direct evidence on the processes young children use when deciding it is safe or unsafe to cross a street, although children around age five accept different time gaps in traffic less of ten than older children, and they use a larger safety margin (van der Molen, 1977; 1978). Thus, while we do not have a clear understanding for the basis of their judgment, it appears that these children can make a safe judgment when they stop to take the time to do it. Sargent and Sheppard (1974), however, report that a sizable number of seven-and eight-year-olds will cross when there is oncoming traffic present. O1der and Grayson (1974) and Sandels (1970) indicate that children frequently do not attend to relevant factors and that they of ten fail to use proper crossing procedures. Howarth (1971) used filmed data to determine both the acceptance gap and the safety gap of five- to twelve-year-olds. older found that children (10-12 years) accepted smaller gaps, but had larger safety gaps ( 3.5 seconds vs. 2 seconds) than younger children. When an adequate gap appeared, younger children waited longer before accepting it. Further, groups of children accepted smaller gaps than individual children. Given this evidence, it seems safe to make a conservative judgment and conclude that while children may have a moderate capability to make gap acceptance judgments, they often fail to take the time to gather the required information and to make a considered judgment. 
Vinje (1978) reports on two studies which relate to this topic, although they did not use naturalistic environments. Gunther and Limbourg (1976) found that within the four to nine age range, there was developmental improvement in the ability to have a doll cross a traffic stream on a small-scale model. Erke (1975) used slides with the aged, other adults, and nine- to ten-yearolds, and found that the elderly used the largest gaps, then nine- and ten-year-olds, while other adults accepted the smallest gaps. Smallest distances for the chlldren involved small cars; for buses and trucks, they chose larger distances. Since there were slides, it is not known whether the children could have used cues other than vision to determine that small cars were not really further away, and that smaller gaps were not warranted.

Mckelvey (1978) has had some success using a discrimination training procedure to teach elementary school chlldren (grades $K-6$ ) to detect safe road crossing intervals, although he acknowledges that thus far no one has been fully successful in such attempts. He does provide evidence that elementary school children can be taught to recognize safe and unsafe intervals, commit those impressions to memory, and recall them in a test situation. They do not, in his opinion, need to perform a more sophisticated integration of speeds and spaces or gaps with his training procedures. Yeaton and Balley (1978) included "watch vehicle distance" as one of six elements of their successful training program so there is further evidence that elementary school children can be taught this skill successfully. Mckelvey does have a number of suggestions for improvements to his procedures, so more effective training procedures for this age range may soon be developed.

\section{I. Route Planning}

Parents and educators also need to be concerned about the (in)ability of preschoolers to plan a route before they depart as pedestrians. There is evidence that kindergarten children, at least, are of ten left to their own devices when it comes to trips to school (Reiss, 1975). Sargent and Sheppard (1974) report that even nine-year-olds have difficulty in finding a safe place to cross a street. Younger children do not even bother to look for such a location; they usually take a straight route to their destination. Boyle and Gilhooly (1972) found that six-year-olds had trouble finding a safe route at a 
crossing junction in a real traffic situation, while Gunther and Limbourg (1976) report similar findings with six-year-olds in a model track. This latter study also found that four-and five-year-olds were very limited in this regard, more so than older chlldren and that the youngest children of ten chose simply the shortest route.

Thus, materials afmed at the preschool population will have to impress upon adults the necessity to plan and select routes for their chlidren, since the children are incapable of selecting safe routes on their own.

\section{SUMMARY}

Materials developed for this age range must take into account all the limitations discussed thus far. Children possess many of the visual system capabilities adults possess, but their ability to process information provided by the visual system is limited during the preschool period. Sensitivity to sound continues to develop through and past the preschool period, but again the prime concern is the limited processing ability of preschoolers during this period. While infants can localize sounds in simple situations, there is no evidence that preschoolers can do so in a traffic situation, nor is there evidence that they can adequately detect and process the speed, direction, and distance of moving vehicles. One reason for these failures may be the limited attention span of preschoolers; they may simply attend to other less relevant aspects of the environment. They are also limited in their ability to comprehend spatial relations, to determine where they are vis-a-vis vehicles and other elements of the traffic environment, and their understanding of causality is minimal. Preschoolers also have a short-term memory which has not fully developed, they require longer reaction time than adults, and they are still trying to gain mastery of their motoric system. They are subject to very impulsive behavior, and they have had different perceptions of $r$ isk than adults.

In addition to their limitations in the traffic situation, preschoolers also face deficits in an instructional setting that impacts upon their ability to learn traffic safety skills. Their memory is still in the process of developing, they have minimal understanding of rules, and they do not 
generally understand that left and right are directions related to their own bodies. Further, they are of ten confused by instructions they do not understand, but they do not know how to indicate this confusion. The vocabularies of preschoolers are limited; they are confused by words which have double meanings, negation or logical connectives, and they fail to understand many traffic terms. Signs and signals in the traffic environment of ten confuse preschoolers. They exhibit minimal evidence of the ability to make safe crossing judgments, and they need extensive help planning safe routes as pedestrians.

Materials developed for preschoolers, therefore, need to be tallored to the known developmental capabilities of the preschool child. By addressing the child where he or she is developmentally, the educational process can take effective root. 


\title{
3 \\ SOCIAL ANO PSYCHOLOGICAL CHARACTERISTICS OF PRESCHOOL CHILDREN
}

\begin{abstract}
Preschool children's behavior in traffic can be further explained by examining their psychological and social characteristics and analyzing how such characteristics might affect their behavior in traffic. This chapter is intended to present an overview of the preschool child's emotional and social makeup. It explores such characteristics as the preschool child's sociability, impulsivity, emotionality, difficulty in separating fantasy and reality, tendency to imitate others, and curiosity. It shows how the preschool child's developmental need to explore the environment and become more independent leads the child into conflict in traffic.
\end{abstract}

This chapter is divided into sections, each of which presents a social or psychological characteristic of the preschool child, and a summary, which reiterates the points covered and suggests appropriate ways for responding to the child's social and emotional needs while still protecting him/her in traffic.

I. SOCIAL GROWTH DURING THE PRESCHOOL YEARS

During the preschool years children become increasingly interested in other people. Their circle of social contacts expands from an initial bond with the primary caregiver to members of the immediate family and outwardly to neighbors, relatives, and visiting friends of the family. The beginning of this process is shown through Bowlby's (1969) and Ainsworth's (1973) studies 
of maternal-child relationships, which demonstrate that it is critical for an infant to form an attachment or bond with the primary caretaker. These early bonds enable children to develop trust and interest in other people later on. Research (Shirley \& Poyntz, 1941) shows that children ages two to seven gradually become less upset over separation from mothers. Preschoolers tend to become more dependent on their peer group--and less on their parents--with age (Bandura \& Walters, 1963). Gradually children move more and more into the company of their peers.)

Learning how to interact with other people is one of the consuming interests of the young child. In fact, gaining social skills is one of the major developmental tasks of early childhood. This process starts at birth. The infant's cries, interest in faces, and desire to be held are the earliest indications of developing social skills. This early interest multiplies during the preschool years. Children this age will go to great lengths to seek out or be noticed by other people. Brazelton (1974) describes a toddler who dances, uses uncharacteristic language, and falls down (i.e., shows off) in order to gain his mother's attention. Brazelton (1974) also cites similar examples of verbal and nonverbal tactics that toddlers use to communicate with others: establishing eye contact, exhibiting outlandish behavior, and running. Stone and Church (1968) make observations of preschoolers who initiate both approprlate and inappropriate behaviors to gain acceptance or assume leadership within a group of other children. Throughout early childhood literature numerous accounts of the preschool child's increasing desire for peer company are documented.

With increasing age and experience, preschool children develop the abtlity to play with other children. Their play patterns evolve from solitary to group play (Eckerman, Whatley \& Kutz, 1975). During the early years most toddlers initiate solitary or parallel play. Solitary play (i.e., by himself/herself) is usually initiated within close range of any adult to whom the child can go from reassurance or help. Parallel play refers to two or more children playing side by side engaged in unrelated activities, but watching, occasionally talking, and sometimes unconsciously imitating the other (Breckenridge Murphy, 1969). Associative play refers to a kind of 
group play wherein each child engages in his or her own activity side by side with other children who are involved in similar activities. In other words, in associative play, children play near each other and form a kind of group but do not cooperate on a single activity. During their fourth or fifth years most children begin to participate in a more organized and complex $k$ ind of play called cooperative play in which they work on the same project or game and share ideas. While preschool children's patterns of social interaction generally begin with solitary play and move to parallel, associative, and cooperative play (Parten, 1932), preschool children of all ages sometimes engage in solitary or parallel play. For example, a four-year-old might play all by $h i m / h e r s e i f$ making a dam in a ditch by the side of the road, while a two-year-old might happlily play next to a group of school-aged children who are playing ball in the road. However, the general trend reveals older preschool children seeking out other children, coordinating activities and assigning specific roles. Such behavior represents a significant change from earlier somewhat disorganized and haphazard associations with other children (Biehler, 1976).

This increasing ability of the child to get along with other children is essential to maturation. Interaction with other children provides opportunities for children to gain social skills such as showing consideration and sympathy, communicating effectively, sharing, and asserting one's rights in appropriate ways (Hymes, 1963). It may be the case that interaction and conflict with peers (and others) gradually helps children learn how to take another person's perspective. Such skills are essential for social life--and traffic situations.

Unfortunately, while the preschool child's increasing sociablitity and social skills contribute to his/her overall development, they may also contribute to his/her danger in traffic. For example, children's strong interest in others may cause them to act in dangerous ways. The toddler may run into the street to get his mother's attention. The four-year-old may dart across the street without even a glance at traffic in order to get to her friend. Peer pressure which starts during the later preschool years may cause a child who ordinarily never crosses the street to run down the sidewalk and 
across the road in a pack of preschoolers. Sandels (1974) reports that children are more concerned with crossing the street to get to a group of friends than scanning for oncoming traffic. Her (1974) analysis reveals that in 56 out of the 182 accidents studied, children's dart-out accidents appeared to have a social cause. She describes two such accidents in the following manner:

A three-year-old runs out from a yard without a gate to meet his father, who is getting out of his car across the street.

A five-year-old is out with his friends. The children are standing at the roadside in front of the house where they live. They have agreed to cross the road to get something at the house of another friend. One of the boys crosses the road, the accident-child stops to let a couple of cars pass then he tries to catch up with his friend, but collides with a car. (p. 9)

\section{THE ROLE OF PLAY IN THE PRESCHOOLER'S DEVELOPMENT}

Social play is the activity at which preschoolers spend the majority of their time (Hetherington \& Parke, 1979). Hohmann, Banet, and Weikart (1979) identified several types of play in which preschool children engage:

- Exploratory play in which children use all the senses to discover the properties of materials such as cars, dirt, water, or pavement, or in which children discover their body parts and how they can move their bodies in space;

- Play in which children arrange and rearrange objects or behavior according to certain patterns, e.g. zig-zag back and forth on a street or draw a chalk design on the sidewalk or road; and,

- Representational play in which children (1) imitate other people's words or actions, (2) use objects to make models of real objects, people or events (e.g., making a model of a carl, (3) use actions, words, and objects to role-play (e.g., pretending to drive a car in traffic), and (4) use highly abstract symbols such as words and numerals (which bear no resemblance to the real object) to symbolize or tell a story about places, people, and events that children have experienced.

Children are seen engaging in all types of play near and in traffic. Sande 1s (1974), for instance, cites instances of children pretending to be policemen, swinging around a lampost, and running back and forth in a 
pedestrian crossing. Preschool children in traffic are of ten seen engaging in the kinds of word play described by Chukovsky (1963) when they invent chants, songs and play games such as "Duck-Duck-Goose" and "Miss Mary Mack" on the sidewalk. Some preschool children may even be drawn into the organized games of elementary school children.

All the forms of play mentioned above are critical to children's growth and development for these reasons:

- Play increases children's large and small motor coordination (Chaloner, 1963). The toddler walking up and down stairs and the preschooler building with dominoes are examples of play being used to increase large and sma 11 motor development. Bruner (1974) postulates that play helps children to master a series of behavioral tasks: "What appears to be at stake in play is the opportunity for assembling and reassembling behavior sequences for skilled action" (p.53). Bruner cites Jane van Lawick-Goodall's research on primate behavior to show how imitative play helps the young to master adult behaviors. Van Lawick-Goodall's research demonstrated that without imitative play and without the role model, the young have great difficulty mastering adult behaviors. This has obvious implications for pedestrian safety education in that young children need opportunities to imitate and role-play correct adult pedestrian behaviors.

- Play enables children to work through anxieties and fears and to regain emotional stability (Isaacs, 1972); Cass, 1971). Cass (1971) recalls how British children who spent fearful nights in bomb shelters during the Battle of Britain had acted out these scenes in their play. When the bombing ceased and some of the terror had passed, children's needs to role-play such scenes decreased. Clearly, says Cass, role-play was a mechanism for helping children to cope with their fear and uncertainty. Role-play allows children to learn and express emotions such as fear, anger, and hostility in safe and acceptable situations. It helps children deal with an anxiety-producing situation such as crossing the street in a safe and relaxed situation.

- Play helps children to learn and experiment with a variety of roles. Preschool children are often seen pretending to be a mother, father, or baby. They also pretend to be busdrivers, police officers, and shoppers. Play gives children opportunities to practice behaviors that they observe people in the traffic environment carrying out--such as as scanning for traffic, stopping at the curb, and beeping a horn. Role-play gives children opportunities to practice using social skills such as sharing and showing consideration for others, which are essential in the traffic environment. In role-play situations children determine and abide by their own simple rules and expectations (Bruner, 1974). This helps them to understand the 
concept of rules and prepares them for observing rules in traffic. Role play also helps children begin to decenter (i.e., see the world as others see it), an important skill for pedestrians, drivers, and bicyclists in the traffic environment.

- Play also contributes to children's intellectual development. It provides them with opportunities to learn in the ways they learn best--through action, through all their senses, through repetition, through experimentation, through their own interests, according to their own level of development and according to their individual pace of learning (Hohmann, Banet \& Weikart, 1979). Giving an example of play's role in the learning process, Bruner (1974) cites a study which he, Sylva, and Genova did at Harvard University with three to five-year-olds. A large group of children were divided into five groups: (1) those who played with a set of materials; (2) those who observed adults perform a specific task (fishing a prize from a box); (3) those who observed an adult perform only certain parts of a task; (4) those who were given instructions and were allowed to manipulate the materials; and, (5) those who received no training. The group of children who played with the materials performed just as well as those who observed the complete solution and significantly better than other groups. Children in the "play" group were noted for their concentration, perserverance, and low level of frustration.

Children who play in the traffic environment are frequently at risk. Sandels (1974) reports that children at play are by far the largest group of victims of traffic accidents. According to Sadler (1969), children who play in streets are more concerned with the play aspect of their behavior than the fact that they are in potential danger. A study by Thackray and Dueker (1983) likewise found that most dart-out accidents were precipitated by play related activities. It is ironic that the very qualities children develop through play--social ski11s, creativity, concentration, perserverance--are not utilized to the benefit of children's safety in traffic when they are playing in traffic. The challenge to parents and educators is to provide avenues for children to develop these qualities through play and help children to use their newly developed skills to increase their safety in traffic.

\section{EMOTIONAL DEVELOPMENT IN THE PRESCHOOLER}

A third characteristic of preschool children is their tendency to be ruled by their impulses (Ross \& Seefeldt, 1978). Sandels (1974) writes, "The youngest ones have no ability to resist sudden impulses; and all the way up through the ages that are dealt with in this study one can notice that this 
ability increases very slowly, but the children have many relapses" (p.17). Children's impulsivity is not surprising considering their egocentrism and lack of self-awareness. In their eyes every desire is a need. Because of their limited sense of temporal relations and their lack of internal controls, any wait for something they want is difficult and frustrating. Preschool children's lack of self-awareness and inability to think abstractly about their own thinking processes renders them incapable of realizing that they are acting impulsively.

Preschool children also tend to be highly emotional and volatile in their expression of emotion. Hur lock (1956) characterizes preschool children's emotions as brief, intense, and transitory. A child may be ecstatic one minute and in tears the next. Children react emotionally to all of the stimuli in their environment. An ant on the sidewalk, a crack in the road, and a bright red car are capable of arousing a preschool child's emotions. Jersild (1968) contends that children's emotions can be aroused by anything that furthers or threatens the gratification of children's desires, their motives, or anything that blocks or promotes their desired activities. Thus, the appearance of an ice cream truck, the loss of a ball into the street, or the sound of an ambulance siren may have a strong effect on preschool children. Why preschool children respond so emotionally is a matter of conjecture. According to Piaget (1972), young children truly believe that objects have thoughts and feelings of their own. Such a theory helps to explain why preschool children become so upset when a favorite toy such as a teddy bear is left in the road. Another theory is that children use certain objects such as blankets, teddy bears, or a parent's scarf as substitutes for their parents, and therefore equate loss of one of those articles with the loss of their parents (Spock, 1976).

A third theory for why children become so emotional relates to the way they channel or control their emotions. Preschool children are still learning how to release the tension caused by the build-up of emotions. Whereas infants react in a rather ungeneralized way to stimuli, preschoolers develop emotional patterns and practices that are highly differentiated (Helms \& Turner, 1976). The preschooler expresses such emotions as joy, sadness, anxiety, fear, and jealousy. Preschoolers are able to express these emotions 
through various means. They express emotions physically through body posture, facial expressions, and action. They skip and dance out their happiness. They hit and cry to release anger and frustration, for example. Increasingly, they are able to use language to express their feelings and make their wants known. Often words fail, however, and they resort to physical means.

Despite their increasing mechanisms for expressing and releasing emotion, preschool children have difficulty controlling and dealing with their emotions. They hit when they know they should not. They take toys that belong to other children. In these and other situations, they have difficulty restraining their impulses to do and get what they want. Such impulsiveness can subject preschool children to danger in traffic, as Sandels' (1974) studies confirm. Children will run across the street to meet their grandparents, dart out into traffic in a game of tag, or run out into the street on sudden impulse.

The intensity of children's emotions also predisposes them to accidents (Hur lock, 1956). While preschoolers are better able to release their emotions through a number of avenues of expression, they still find it difficult to behave rationally and safely when their emotions are high. Sandels (1975) comments:

When small children become greatly aroused - whether it be a question of happiness, sorrow, anger or other emotions - they become so taken up with their feelings that everything eise ceases to exist for them. (p. 45)

As infants, children's emotions are known to bulld to the point where all emotions, even those produced by pleasant experiences, become disagreeable and painful, thereby causing the infant to cease activity, breakdown, and cry (Stone \& Church, 1968; Baldwin, 1955). Preschool children are affected by a build-up of emotion in a slightly different manner, but their activity is also impaired by intense emotion. Hurlock (1956) states that children's behavior is less organized, their play less constructive, and their physical coordination less intact when they are extremely emotional: 
Emotional tension results in rigidity, which is reflected in a narrowing of the perceptual field and a decreased ability to respond to environmental clues, thus causing the child to act on the basis of minimal information. (p. 306)

Hur lock notes that heightened emotionality in babies and young children appears at certain predictable times of the day, peaking at periods prior to scheduled times for eating and sleeping. The implications of Hur lock's statement are that prior to mealtime and sleep (e.g., during the afternoon) preschool children tend to be most emotional, and partly as a result, are less able to cope effectively in traffic situations. Such implications are reflected in the research findings specified in Chapter 1.

Brazelton (1974) describes how the bufld-up of emotion in toddlers often gives way to temper tantrums and negativism. It follows that toddlers, who are likely to have temper tantrums and disobey parents, would be at risk in the traffic environment.

Despite the fact that children's emotionality places children at risk in traffic, preschool children's emotional natures should not be suppressed. Emotionality in young chilidren is reflective to their developing maturation. Jersild (1968) concludes that the preschool child's heightened levels of perception and self-esteem contribute to the maturation of emotional expression. In other words, increased experience dealing with powerful emotions enables children to internalize their feelings and express them more successfully. The ability to feel and articulate various gradations and types of emotions is a prerequisite to lasting attachments. As testimony to that fact, bursts of temper and quarrels have been reported to occur more frequently among preschoolers who are able to form friendships (Biehler, 1976). Temper tantrums, fights, exuberance, joy, fear, and seif-confidence are all emotions that preschool children need to experience. Only by experiencing such emotions can young children learn to regulate and channel them and eventually learn to walk safely in traffic despite intense feelings. 


\section{THE DEVELOPMENT OF IMAGINATION}

The later preschool years, ages three, four, and five, are the years when children become involved in fantasy. They remember things that have happened and begin to imagine what might happen. Sometimes they daydream. Sometimes they act out what they imagine in role-play or play with miniature models of the world (such as dolls, doll houses, toy cars, and blocks). In other words, they incorporate fantasy into their play. They also use language to express what they imagine.

Although preschool children use fantasy in their play, they do not understand what fantasy is. As Stone and Church (1968) write:

Such a view... assumes tat reality and fantasy are two distinct realms, which is not altogether the case for the preschool child, for whom fact and fancy are likely to be interchangable. The line between the two realms becomes more sharply defined during the preschool years...but feelings, thoughts, and wishes continue to flow out into the influence the objective world, animating and coloring and shaping it in ways that adults may find surprising. (p. 291)

As stated earlier, preschool children may attribute iffe to inanimate objects (Piaget, 1972). According to the preschool child, rocks, trees, and clouds think and behave as people do. In other words, the child subjects his or her own thoughts and feelings to others. There is little separation between "I" and the environment, between children's thoughts and reality. Children of ten believe that dreams are actual experiences that occur in real space (Stone \& Church, 1868; Piaget, 1972). They of ten believe that television shows, movies, and storybook characters are real and that even their magical feats are real (Stone \& Church, 1968). They also have difficulty differentiating between symbols and their referents (Elkind, 1970). In other words, saying or thinking something makes it happen.

The child's inability to differentiate reality from fantasy may place $\mathrm{him} / \mathrm{her}$ in a precarious position. Sandels (1974) and the OECD Special Research Group on Pedestrian Safety (1978) have, among others, concluded that preschool children are often living in the world of fantasy when they are in 
traffic and have difficulty separating play from fantasy. A child who believes that objects have thoughts and feelings may unconsciously think that the physical environment will protect him/her. For example, the child may think that the pedestrian crossing lines will automatically stop any cars from hitting him when he runs across the crossing. Also, that child may dash out into traffic to protect an object such as a doll or mitten or scarf that has fallen in the road. The child, in Stone and Church's (1968) words, "may nourish a firm conviction that he can magically influence events by an application of his will, just as he sees an adult 'making' the car go" (p. 291). Children may feel that they can behave in traffic with the same super powers revealed by television and comic book heros. Children may think that drivers will possess the same compassion and omnipotence that they have in stories and in dreams. Children have little perception of the "realities" of traffic. In this regard, Sandels (1974) writes: "It is evident from this study, as from previous ones, that the children in all age groups concerned, are often living in a world of play when they are in traffic, both as pedestrians and as bicyclists" (p. 17). Sandels (1974) also reports that many children have an almost magical belief that pedestrian crossings will protect them from vehicle accidents, regardless of the amount or speed of trafic.

Yet, for all the dangers young children encounter due to their confusion between fantasy and reality, their use of fantasy is essential to their cognitive, social, and emotional growth. Like play, fantasy provides the child with an avenue for the generation of novel solutions to complex problems. Fantasy enables the child to maintain control over his/her own environment. In time, from comparing dreams to reality, from testing reality, and from becoming more aware of the creative process, children begin to become aware of the existence of their own thoughts (Piaget, 1972). Children begin to realize that they can plan ahead and think of steps they need to take in order to accomplish a task. Such thinking is essential for safe road crossing.

\section{IRRATIONAL FEARS IN PRESCHOOL CHILDREN}

Preschool children's actions may be triggered by irrational fears which may impede their ability to cope with traffic. During the early preschool years children often become afraid of noises, strange people, falling, and 
loss of support (Jersild \& Holmes, 1935). Hur lock (1956) adds animals, dark rooms, and high places to the list of early fears. Gradually these fears subside. Breckenridge \&urphy (1969) attribute the wane in these fears to young children's increasing ablitities to explore objects and understand them. Sluckin (1979) describes a "discrepancy" or "incongruity" hypothes is developed by Hebbv, Schaffer, Kagan, and others who posit that through experience children gain a frame of reference (internal images) so that the objects that once were frightening no longer frightened or upset them.

0lder preschool children, caught up in play and fantasy, fear imaginary things. They worry about the dark, imaginary creatures (e.g., monsters) and the threat of harm from traffic, deep water, and fire (Kersild \& Holmes, 1935). They often fear that their own hostility will cause an accident and injury to themselves or others (Fraiberg, 1959). They fear illness, pain, injury, and accidents (Petrillo \& Sanger, 1972; Plank, 1971; Hardgrove \& Dawson, 1972). Where do such fears come from? Hur lock (1956) surmises that children develop fears through prior unpleasant experiences, imitation of their parents' fears and from books, television, and stories. Fraiberg (1959) and others (Hymes, 1974; Wolman, 1978) belleve that fears are accentuated because preschool children have such difficulty separating fantasy from reality.

When children are afraid, they may be further endangered in traffic situations. Just as with any kind of intense emotion, fear may cause a child to be less observant in traffic. For example, a child worrying about the consequences of dreaming that his/her mother died may run across an intersection without looking to get home as soon as possible to make sure that the mother is still alive. The build-up of tension from fear may cause a child to exert a lot of unneeded physical action to release tension. This tension may cause $\mathrm{him} / \mathrm{her}$ to run rather than walk and lose focus rather than concentrate. in traffic situations.

The development of fear, while it places children at risk, is an important and necessary development for the child. Without some measure of fear and caution, man would not be able to survive. Fear in truly dangerous situations is entirely appropriate. As with other emotions, however, children need to 
learn how to handle fear, how to assess whether fear is based on real or imagined occurrences, and how to steady themselves and take carefully planned action during potentially dangerous situations.

\section{PARENTS AS MODELS}

Starting in infancy children begin to imitate other people--especially their parents. Felsenthal (1972) notes that children imitate their parent's attitudes and feelings, their words and their actions. As teachers of pedestrian safety, parents model their attitude towards safety, risk taking, injury, life, and other people. They teach children language associated with the traffic environment and inculcate pedestrian habits which make an indelible impression on their children.

In all aspects of learning (not just pedestrian safety), parental behavior provides a strong influence on the child's subsequent development (Stone \& Church, 1968). Many studies have shown (Bandura, 1967; Baumrind, 1971) that parents have a powerful effect on their children's growth and development.

Children imitate adults for a number of psychological-social reasons (Secord \& Backman, 1964). The child maintains parental affection by behaving in a similar fashion to the parent (Kagan, 1958). The child develops a sense of identity by learning to distinguish between people who act like "us" and people who act like "them" (Parsons, 1955). The child may identify with an adult out of fear of punishment (Secord \& Backman, 1964). The child may identify with an adult because of the promise of rewards or by observing that adults who act in a particular way are rewarded (Hetherington \& Parke, 1979). In fact, children are most likely to identify with any adult who is powerful and nurturant (Bandura, Ross, \& Ross, 1963; Mussen \& Parker, 1965).

Young children will imitate adult behaviors even in the face of contradictory verbal messages (Banduar (1967). In other words, in mixed messages set out by adults, children of ten detect behavior to be the stronger clue. Hetherington and Morris (1978) described the process of identification this way: 
A 'do as I say, not as I do' approach to socialization is ineffective. If the child sees a church-going, platitude-spouting, moralizing parent lie about his golf score, cheat on his income tax, bully his children, and pay substandard wages to his help, the child may emulate his parents 'behaviors rather than his hypocritical words. (p. 9)

Bandura (1967) likewise describes children's abilities to imitate parents' actions:

Research bearing on modeling processes demonstrates that, unlike the relatively slow process of instrumental training, when a model is provided, patterns of behavior are rapidly acquired in large segments or in their entirety. (p. 43)

Hetherington and Parke (1979) concur with Bandura that extensive imitation of parental behavior occurs whether or not the parent knowingly tries to shape the child's development.

Parents' mixed messages may add to children's difficulties coping with traffic. Foote (1961) states that parents unconsciously contribute to their own children's accidents by modeling unsafe ways to deal with dangerous situations such as traffic. Sandels (1970) observations of children crossing streets alone, with other children, and with adults attest that children display the worst traffic behaviors when they are in the company of adults. In most cases children are allowed to run ahead or behind the parent and another child. An opportunity to model correct pedestrian behaviors and call the child's attention to those behaviors is clearly missed. Also parents have been known to hurry children to cross when the traffic light is changing from green to red (Sandels, 1974).

\section{THE DEVELOPMENT OF TRUST}

Young children generalize their feelings for parents to adults in general. Being egocentric, young children feel that other adults are an extension of their own world and their home environment. Children of ten feel that all adults are omnipotent, nuturing individuals just as they regard their own parents. 
The child's perception of adults as harbingers of good may place $\mathrm{him} / \mathrm{her}$ in danger in traffic. As discussed previously, Sandels (1964) feels that because children do not understand causality, they cannot understand that drivers in traffic are not automatically able to protect them from harm. She says that young children assume that adults will always be kind to them and that drivers will be able to stop instantly if a child is in danger. Consistent with this finding is a complementary finding that young accident victims rarely blame an adult for causing an accident--even in the face of contradictory evidence. The Skandia Report (Sande 1s, 1974) describes this phenomenon:

There were no cases where the children blamed their parents or other supervisors, not even when it is quite obvious that they had let the children play in a dangerous environment when they were much too young for it, or when the supervisors in the company of the child had behaved negligently. (p. 43)

\section{THE DEVELOPMENT OF CURIOSITY}

Preschool children are known for their curiosity. Everything in the environment is still new and interesting to them. The traffic environment is especialiy interesting to them because of its movement, the variety of objects, and the people in it. Young children are interested in different kinds of road surfaces, grates, lights, vehicles, noises, people, and animals. Unlike adults, preschool children do not just look at things that interest them; they actively explore the traffic environment with all their senses. They will stop to watch water running into gutters and put their hands in the water to feel the current. They listen to ice cream trucks and sirens and try to imitate their sounds in play. Watching another child roll down the sidewalk on a Big. Wheel, a preschool child will run after him and experience for herself what it feels like to move that quickly in space. Not only do preschool children explore objects and people within the traffic environment, they also explore how they can move their bodies in space. They will run on cobblestones to master the skill of running on bumpy surfaces. They will swing around lampposts to explore the sensation of moving in 
circles. They will zig-zag along an alley to explore moving in such a narrow, long space. They will practice walking along the curb. Such motor exploration improves their motor coordination and teaches them about parts of the traffic environment. While parts of the environment interest them, children are especially interested in people, living animals, and moving vehic les in the traffic environment.

Curiosity about the traffic environment of ten leads small children into dangerous situations. Youngsters catch sight of something interesting on the other side of the road and run across to investigate it more closely. For instance, Sandels (1974) reports the accident of a six-year-old who ran across the street to get a better look at a military parade. Many traffic accidents are caused by children running across the street to see the ice cream truck or just running out into traffic because of a chasing game.

Children as young as two and three are most susceptible to danger brought on by curiosity and a lack of understanding of what is dangerous (Youshiyuki, 1978). They will walk right into the road to pick up an interesting pebble they see or explore the hub cap on the outside wheel of the car. Small children are completely unaware that they can be hit by moving vehicles. Chaloner (1963) comments on the young child's handicap in traffic in this way:

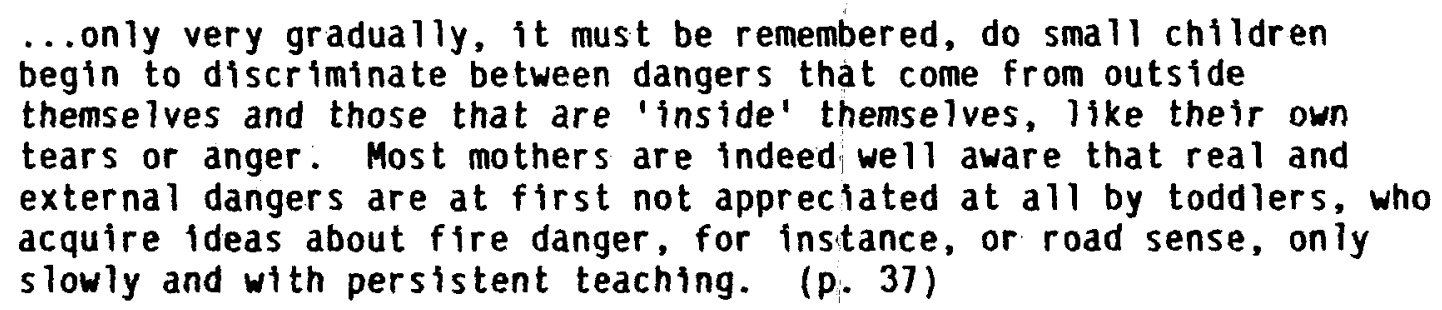

Yuoshiyki (1979) states that small children will only learn to be more careful through experience and increased insight into why a situation is dangerous.

Although older preschool children have a better understanding of the danger of the traffic environment, they are still at risk. Because of their intellectual limitations, they are unable to attend to traffic while they focus on an aspect of the traffic environment about which they are curious 
(Sandels, 1974). As stated earlier, their confusion between reality and fantasy of ten leads them to believe that they will magically be kept from harm while they investigate the traffic environment. Thus, curiosity may lead children to dart across the street in total disregard for oncoming traffic. Curiosity may also lead children to attempt maneuvers in traffic which are beyond their capabilities.

While it leads young children into danger, curiosity in young children should not be suppressed for developmental reasons. It is through exploration of their environment that young children learn (Hohmann, Banet and Weikart, 1979). Curiosity is needed for the development of intelligence, sociability, and all aspects of child development and growth (Tanaka, 1965); it if it unduly suppressed, growth is hampered (Yoshiyuki, 1978).

\section{CONCLUSIONS}

In summary, preschool children tend to be curious, impulsive, emotional, imaginative, fearful, playfu1, irrationa1, sociable, trusting, and imitative. Most of these qualities enable children to learn about themselves and the world around them; suppressing these qualities would inhibit children's total development. However, preschool children need safe places to explore and express themseives in addition to protection in traffic. They will best learn the skills and principles of pedestrian safety through a creative instructional approach which makes use of their curiosity and imagination and provides them with opportunities to learn in the ways which are most meaningful to them--through discovery, play, imitation, and firsthand experience. 


\section{4 \\ CURRENT PEDESTRIAN SAFETY PROGRAMS FOR PRESCHOOL CHILDREN}

Concerned by pedestrian-auto accidents which befall young children in disproportionate numbers, various public and private organizations in the United States have sponsored pedestrian safety programs in hopes of teaching children safe pedestrian behavior and thereby preventing and/or reducing such accidents.

This chapter contains a review of existing pedestrian safety programs in the United States as of Apr 111980 that address the preschool ages. The first part of this chapter summarizes these programs. It presents:

1) A history of pedestrian safety education for young children in the United States;

2) A discussion of the present status of preschool pedestrian safety education:

3) Descriptions of extant preschool pedestrian programs;

4) An overview and listing of concepts taught;

5) A discussion of pedestrian instructions given;

6) Commentary on and a listing of teaching methods/materials used; and,

7) A discussion of some of the contributions which these program have made.

8) Forty-five programs that are designed for in-school use are described in detall at the end of this chapter. In addition, seven privately developed programs specifically targeted for preschool audiences are highlighted.

I. A HISTORY OF PEDESTRIAN SAFETY EOUCATION

There has always been a need for pedestrian safety education for young children, but the need has become more critical as a result of increased 
traffic volume, faster vehicle speed, changes in the environment, and changes in family life-style.

Tracing the history of pedestrian safety education, one finds that school children took lessons in safety education even during the time of hickory sticks and inkwells; safety lessons are known to have been taught as early as 1845. In fact, McGuffey's Eclectic Readers, published in 1879, contained several safety admonitions (Stack, 1966). Safety lessons taught during that era, however, contained only general safety instructions. Pedestrian safety education as a unit of instruction separate from home, fire, and other forms of safety instruction did not appear until much later, not until after the automobile had become a primary mode of transportation and a great many auto/pedestrian deaths had occurred.

Introduced in the United States at the turn of the century, the automobile and its hazards were gradually discovered by the general mix of traffic, and between 1910 and 1920 automobile traffic became recognized as a safety problem. In fact, the need for safety education was one of the topics of discussion during the yearly meeting of the National Safety Council in 1914 (Stack, 1966).

Several groups mobilized to try to prevent children's traffic accidents. One of the first to address the traffic problem was the city of Tacoma, Washington, which instituted school safety patrols in 1913 (Berger, 1975). Soon thereafter, cities across the county--Newark, Roselle Park, and Paterson (New Jersey), Woonsocket (Rhode Island), Philadelphia, Washington, D.C., Warren and Canton (Ohio), Chicago, and Seattle--developed their own school safety patrols (Stack, 1966). These patrols, of ten sponsored by local community organizations, the school district, and the local law enforcement agency, represented the beginning of a beneficial liaison between private agencies, the police, and public schools. In 1922 the American Automobile Association (AAA) developed standards, guidelines, and procedures for School Safety Patrols and spread the School Safety Patrol System throughout the United States by means of AAA's nationwide club network (Lafond, 1980). Student safety organizations such as junior safety councils also became established in elementary schools about that $t$ ime. 


\section{I.A. The Growth of Safety Education}

The 1930s saw a period of growth in traffic safety educational materials. Many organizations, particularly those in the private sector, made important contributions which precipitated this growth. AAA, for example, began to produce safety educational materials for its members during the early thirties (Weiss, 1980). The National Safety Council (NSC) also produced safety materials for its members and, under a Federal charter, produced safety posters and other such materials for safety campaigns for nonmembers. From the public sector, individuals within the driver education movement (which began in the twenties and gained momentum during the thirties) produced materials instructing drivers in practices for dealing with pedestrian traffic. These materials, however, were written totally from the driver's point of view; they were not written with the primary purpose of teaching safe pedestrian behavior to pedestrians. Other educational materials included films on traffic safety and visual aids which were introduced into the schools during the thirties (Lafond, 1980).

Safety education as a course of instruction spread. By 1938, $82 \%$ of the elementary school teachers surveyed by the National Education Association (NEA) reported that they taught safety education. Teaching methods used included discussion, film, lectures by nonschool personnel such as police officers, visits by the school safety patrol, and bulletin board displays (Stack, 1966). For the most part, these educators taught children a set of traffic rules--mostly the "do's" and "don'ts" of pedestrian behavior (Lafond, 1980). Teachers were reported to be the primary developers of safety education classroom curricula; school departments provided little supervision or guidance (Stack, 1966).

A concern was voiced regarding the lack of training for teachers in safety education in the late 1920s. To fill this void, several centers of learning such as New York University's Center for Safety Education, the Teacher's College at Columbia University, and the George Peabody University in Nashville, Tennessee began to teach courses on safety education to teachers during the 1930s. From these institutions came publications such as Education for Safe Living by Hubert J. Stack of New York University's Center for Safety 
Education (Berger, 1975). Other organizations that responded to teachers' need for guidance were publishing companies and AAA and NSC which developed safety education curriculum materials, films, posters, and brochures. Just before World War II broke out, the NEA completed the first comprehensive bibliography of safety education. Another contribution which affected the safety education movement was the 1940 Yearbook, Safety Education which was issued by the American Association of School Administrators (Stack, 1966).

\section{I.B. A Lapse in Safety Education}

After the second world war came the baby boom and the car boom. During the 1950s and 1960s there were more types of vehicles on the road than ever before as more people were driving than ever before. More people were also getting in and out of buses, cars, taxis, and trucks. There were simply more people, especially children, in traffic. Living patterns had changed: populations in northern industrial cities multiplied and record numbers of families began to live in suburban communities and commute into the cities. of course, there were accidents.

Surprisingly, there was a decrease in traffic safety education in the classroom during the 1950s and 1960s. Berger (1975) suggests that the reason for the decline in safety education was that the public considered the original traffic problem "solved" and therefore, generated less support and fewer funds for safety education in the schools. He points out that the rates of incidence for pedestrian accidents per miles driven were lower in the 1950s than they had been in the 1920s and 1930s. Marsh's (1955) analysis of traffic accidents confirmed this. Comparing accident statistics from 1955 and 1937, Marsh revealed that by 1955 the number of pedestrian fatalities were reduced to nearly half as many as were reported in 1937. While these statistics represented an indisputable and noteworthy drop in the absolute numbers of pedestrian deaths, they could not be interpreted to mean that children were no longer in danger in traffic. An analysis of the accident data from the 1950s revealed that 9 out of 10 pedestrians killed in traffic were nondrivers (Marsh, 1955). Thus, groups of nondriving pedestrians such as children were overrepresented in traffic accident fatalities. Clearly, there still was need for traffic safety education. 
Another possible reason for the decline in traffic safety education was that during the 1950s and 1960s the country as a whole placed a low priority on traffic education. America was, at this period in time, up in arms over the spread of communism, agonizing over racism and civil rights, trying to win the arms race, and plotting to be the first to land a man on the moon. Traffic safety education took a back seat to academic subjects such as math and science and to "relevent" issues such as racial integration and the Bay of Pigs. However, traffic safety education, though neglected, was still very much needed, as young children continued to be killed and injured.

Meanwhile, the insurance companies, concerned about the loss of life and damaging physical and psychological effects of traffic injuries and interested in improving public relations, began to take action to reduce actual accident rates. They began to develop traffic safety materials first for their constituents and later for the general public. Aetna, Allstate, Kemper, Metropolitan Life, and Travelers--these were some of the insurance companies which were involved in traffic safety education at this point in time (Lafond, 19801 .

Private industry also became involved in traffic safety education. For example, the American Trucking Association, American Motors, Chevrolet, Ford, Chrysler, and Goodyear Tire and Rubber Corporation produced their own traffic safety materials (Lafond, 1980).

Although law enforcement agencies around the country had been working to increase pedestrian safety from the inception of the traffic problem, during the 1960 s many innovated a new program, the officer Friendly Program, in an effort to improve the police officer's image in the community. This program (now sponsored by the Sears Roebuck Foundation) brought police officers into elementary school classrooms to increase children's awareness of the role of law enforcement officers in the community and to teach traffic safety concepts (Lafond, 1980).

All this time, AAA, NSC, and other private organizations continued their work in traffic safety education. 
Despite all these efforts in traffic safety education, children continued to be hit by traffic vehicles. Experts in the field of driver safety education began to see the need to teach traffic safety education long before the high school years, when students traditionally began driver education. These experts suggested revitalizing the elementary school traffic safety curricula (Lafond, 1980).

\section{I.C. Federal Impetus for Traffic Safety Education}

The next watershed development in traffic safety education occurred in 1966 when the Pedestrian Standard of the Federal Highway Safety Act was passed. Shortly thereafter in 1968, the National Highway Traffic Safety Administration (NHTSA) allocated funds to several State Offices of Highway Safety who had developed safety education programs for elementary schools within their states. This was the time when pedestrian safety education, in its own right, gained impetus. A number of states, Illinois being one of the first, produced curriculum guides which had separate units of study for pedestrian safety. Many of these curricula were developed jointly by state departments of education and offices of highway safety. Some curricula such as New York State's Traffic and Pedestrian Education Systems (TAPES) program were developed by private contractors. As most of these programs were geared to the K-3 age group, many members of the five-year-old sector of the preschool age group now received pedestrian safety education lessons which were approved by a state department of education, a marked change from earlier days when there had been little supervision of safety education taught in the classroom.

There was a significant difference between the safety education lessons in these state curriculum programs and those in the programs of the 1930s. Early programs primarily taught lists of traffic rules. In contrast, the new state curriculum programs introduced children to safety features in the traffic environment and to the interaction of different components within the traffic system such as police officers, school safety patrol, drivers, pedestrians, and bicyclists. Less emphasis was placed on traffic rules in and of themselves and more was focused on helping children to understand the dangers of various traffic situations and the need for responsible safe behaviors. 
The new state curricula also used a greater varjety of teaching methods than had previously been employed. Now children were role-playing how to cross streets and making models of traffic environments whereas before they had been passive learners who memorized safety rules. Many of the persons responsible for bringing about this new trend in safety education came from state universities and organizations within the private sector. Stack from New York University, Kennel and LaFond from Illinois State University, Bishop from Florida State University, Whitney from Casualty Insurance, and Abercrombie, Key, and Marshall from the National Commission on Safety Education played an important role in developing the philosophy of safety education as reflected in the state curriculum materials and other such programs (Kenne1, 1980; LaFond, 1980).

Besides sponsoring traffic safety elementary school curricula during the early 1970s, the NHTSA funded other traffic safety approaches--safety campaigns, police officer programs similar to the officer Friendly Program Described above, and Safety Towns. In fact, the first NHTSA-funded Safety Town was initiated the same year that the state offices of highway safety received funds to implement traffic safety curricula in elementary schools. A short while later, the National Safety Town Center began to develop program guides for teachers and parents in Safety Town programs in hopes of promoting the Safety Town concept (Lafond, 1980).

Meanwhile, groups within the private sector such as AAA, NSC, and the Automotive Safety Foundation continued to produce traffic safety educational materials. The insurance companies' involvement in safety education, however, gradually subsided while media companies such as Film Loops, Inc. entered into traffic safety education (Lafond, 1980).

\section{D. Important Contributions to Traffic Safety Education}

During the 1960s and 1970s there were four major developments which both stimulated and contributed to the development of traffic safety programs in the United States. 
Research on children's behavior in traffic was one development which precipitated and influenced traffic safety education. Researchers such as Sande 1s, Routledge, Cameron, and others mentioned earlier in Chapter 1 of this report alerted program developers to the fact that young children were overrepresented in traffic accidents. These researchers called attention to children's pedestrian behaviors that lead to traffic accidents and helped developers understand some of the reasons for those behaviors.

A second development to influence traffic safety education was the initiation of task analysis, which has been utilized by researchers such as Every, Kennel, and others. Task analysis, as applied to accident analysis, is the technique used to break down a traffic task such as crossing the street into several subtasks or smaller steps. It has helped safety program developers and educators to appreciate how difficult the learning of safe traffic behavior is for the young child. By revealing to educators the many perceptual motor skills needed for safe behavior in traffic, task analysis has helped recent program developers to surpass the efforts of early safety educators who typically taught itttle more than lists of traffic rules.

Research in early childhood development was a third factor which has contributed to recent traffic safety programs. Researchers, scientists, doctors, theorists and educators such as Piaget, Flavell, Bruner, Gesell, and others mentioned in chapters 2 and 3 have contributed much to our understanding of young children. Knowledge of young children's capabilities has begun to alter adult expectations of children in traffic. It has enabled adults to see traffic and traffic training more from the child's point of view, a skill which has in turn helped adults to develop more appropriate and more effective traffic safety lessons.

The development of the NHTSA's accident typing system and countermeasure approach to program development was a fourth contribution to the development of traffic safety programs. The 1971 Department of Transportation sponsored study, Pedestrian safety: The Identification of Precipitating Factors and Possible Countermeasures (Snyder and Knoblauch, 1971) represented the debut of accident typing. Beginning with this study, a system was developed for classifying traffic accidents according to certain types of accidents which 
had similar causes and situations and which were found to occur again and again (e.g., the dart-out, the intersection dash, the vehicle turn/merge). Using this system, analysts extracted from police accident reports information such as the victim's behavior, the driver's behavior, the victim's age and sex, the victim's color of clothing, the driver's sex and age, the type of vehicle, time of day, the day of week, and site characteristics. Such information has enabled analysts to identify more precisely the characteristics of persons involved in each accident type, their behavior, and any other precipitating and predisposing factors which may have directly or indirectly caused the accidents. The classification or typing of a large sampling of accidents has enabled policymakers, planners, and program developers to see trends in accidents among different age groups and to pinpoint traffic sites or situations that appear to predispose persons to accidents. With a deeper understanding of the causes of accidents, communities have been better equipped to take action to prevent or reduce accidents.

The NHTSA's approach to program development has also had an effect on traffic safety program development. Programs based on the NHTSA approach are empirically based and focus on reducing the occurrence of specific accident types. This approach is in contrast to many of the state traffic safety curricula of the early 1970s which taught everything from community helpers to weather to traffic lights. The NHTSA accident typing system and countermeasures approach can be seen as a boon to educational program developers because it has helped them to seek out "the problem" in a more systematic and comprehensive way and has enabled them to develop programs with more clearly defined objectives.

\section{I.E. Advent of Preschool Pedestrian Safety Education}

Prior to the 1970s, the preschool age group was one of the neglected groups in traffic safety education in the United States. Although accident research reports indicated that the preschool population was overrepresented in pedestrian accidents and although accident research studies such as $\mathrm{Or}$. Stina Sandels' (1966) observations of Swedish preschool children's traffic behavior indicated that young children have great difficulty dealing with 
traffic situations because of developmental limitations, few materials were written for or aimed at the preschool age group in the United States prior to 1970 .

Preschool pedestrian safety education began during the 1970 s and is stfll evolving in the 1980s. During the early 1970s the NHTSA began to see a need for research on preschool children's pedestrtan safety. In 1979, having developed empirically based pedestrian programs for the $\mathrm{K}-1$ ? age groups, research and development with in NHTSA expanded its efforts to include the youngest age group in its pedestrian research, and thereupon sponsored the current project, the WITS program for preschool aged children.

Efforts to develop preschool pedestrian programs on the slate level were initiated through the state offices of highway safety. Some of the earliest efforts in safety education for preschool children included New York State Department of Motor Vehicles's Traffic and Pedestrian Education Systems (TAPES) proyram for four to six-year-olds and several Safety Town programs, many of which included preschoolers. The Michigan Office of Highway Safety launched its its Preprimary Traffic Safety Curriculum for Saginaw, Michigan, in 1978. The Connecticut Office of Highway Safety initiated the Preschool Traffic Safety Club (newsletter) in 1980 and the Preschool Education for Safety Program (a classroom program) in New Haven. About the same time, Ohio launched a pilot pedestrian preschool program, and Utah decided to pilot some "safety citics" based on the Safety Town concept.

Aside from efforts sponsored by the NHTSA and State Offices of Highway Safety, private groups such as AAA and the NSC have instituted pedestrian 
programs for preschool children. AAA's Preschool Children in Traffic series was developed in 1976, while NSC's Green Cross Safety Club ${ }^{6 /}$ was initiated in 1978. In addition, many television networks are beginning to produce public service announcements designed to teach traffic safety concepts to young children. Newspapers and magazines are featuring more articles on the dangers of traffic for young children. From the initiation of the above programs and increased literature concerning the plight of preschool children in traffic, it appears that we are entering a period of awareness and support for preschool pedestrian safety education.

\section{I.F. Conclusion}

Since the founding of our country, changes in the environment have forced child pedestrians to cope with more and more complicated traffic situations. Where once a child had a forest or a meadow for a backyard, now he or she may have to cross several driveways, alleys, streets, and intersections to reach a space to play. Where once a horse and buggy seldom came to a homestead, now a steady stream of cars, trucks, mopeds, and other such vehicles pass the apartment and home. Thus, changes in the environment, traffic density, and vehicle speed pose increased hazards to the child in traffic. As the pace of life has quickened, today's child of ten travels to several locations during a day-to school, to day-care, to the supermarket with a parent, to home, to a friend's house down the street, and back home. Although the traffic environment itself has become busier, children are exposed more frequently to traffic situations, and thus are more of ten in danger.

Despite innovations such as traffic lights and median barriers which have been introduced to the traffic environment in order to save lives, children are still vulnerable. In fact, our improved traffic environment is complex and difficult for preschool pedestrians to master. In addition to the difficulties of perceiving moving vehicles and interpreting which way they will move and how fast they are moving, a child must learn to decipher several different abstract symbols such as turn signals, traffic signs, traffic lights, and lines on the road, not to mention bicyclists', drivers', and motorcyclists' hand signals to pedestrians. Thus, the tasks of teaching 
children pedestrian safety concepts and safe pedestrian behavior are an increasing challenge.

The next section will take a more in-depth look at some of the existing child traffic safety programs.

\section{PRESENT STATUS OF PRESCHOOL PEDESTRIAN SAFETY EDUCATION —}

At present there are no completed state, regional, or federally planned pedestrian safety education programs based on empirical data and designed specifically for the preschool age group (ages two to five). Some states do, however, include older preschool children in K-3 traffic safety programs. For example, New York, Missouri, the District of Columbia, and Virginia implement traffic safety programs which include four and five-year-olds in the same programs for children in the early elementary grades. A few states--Georgia, Minnesota and North Dakota--distribute the same safety coloring book lets to preschool and elementary school children. Most Safety. Town and Officer Friendly types of programs that are planned and implemented by states and localities are for older preschoolers and elementary school children. The on ly completed pedestrian program for all children in the preschool age range which has been identified is AAA's Preschool Children in Traffic series.

Several preschool pedestrian programs are, however, currently being developed. As noted, the NHTSA is currently sponsoring the development preschool child safety program for children under the age of six, their parents and their teachers. Known as the Walking in Traffic Safely (WITS) program, this program uses an empirically based child development approach. Encouraging states to plan, develop and implement preschool pedestrian programs, the NHTSAA has in addition, contributed funds to Michigan, Connecticut, and Ohio, which have begun to develop and implement preschool programs. Michigan is in the process of developing the Preprimary Traffic

\footnotetext{
1/This report describes the status of preschool pedestrian traffic safety education in the U.S. during the 1979-1980 school year.
} 
Safety Curriculum for inner city children. Connecticut has two preschool programs which are developing simultaneously--The Preschool Traffic Safety Club (newsletter) from the Connecticut office of Highway Safety, and Preschoo? Education for Safety, a classroom program being developed in New Haven. Ohio has a preschool traffic program being developed and piloted by the University of Cincinnati. In the private sector two preschool projects nearly completed are Dennis Embry's Safe Walking Project, sponsored by the AAA, and the already-mentioned Green Cross Child Safety Club, an effort by the NSC to establish a safety club for preschool children and their parents.

\section{Exhibit 4.1 "Current Status of Preschool Pedestrian Safety Education"} shows where programs designed to teach pedestrian safety to preschool children are being implemented in the states and territories of the United States. The information presented was obtained from the governor's highway safety representatives in each state and territory and the following organizations: $A A A$, the Sears-Roebuck Foundation, Media Intensive Learning Corporation, and the National Safety Town Center. When other programs such as Beltman (by film Loops, Inc.), and Discovering Traffic Safety (by the Automotive Safety Foundation) were mentioned by the governor's highway safety representatives, that information was indicated on the chart. The chart, although not able to provide complete information about the implementation of preschool pedestrian programs in this country, does provide an overview or general picture of the status of preschool pedestrian safety programs in the United States during the $1979-1980$ school year.

\section{TYPES OF PRESCHOOL PEDESTRIAN PROGRAMS}

Preschool pedestrian safety programs are diverse in their approaches to children, teaching, and safety education. Seven types of programs which it is felt are representative of this diversity are identified. These approaches also represent the major types of programs currently being implemented.

\section{III.A. The State Curriculum Manual Approach}

Most of the state traffic safety curricula appear to focus on increasing children's knowledge of pedestrian safety; they are not behavior oriented 
EXHIBIT 4.1 CURRENT STATUS OF PRESCHOOL PEDESTRIAN SAFETY EOUCATION

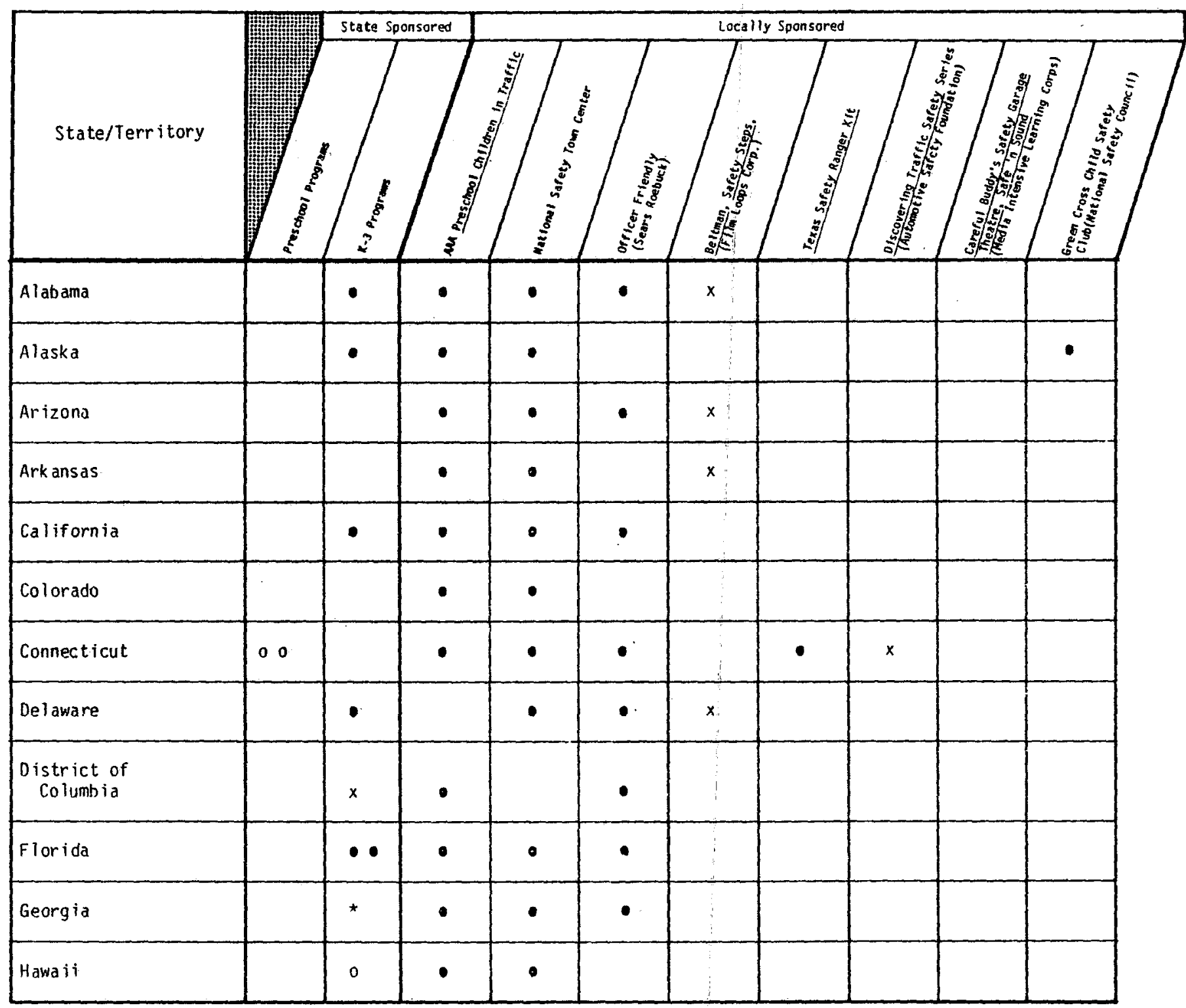


EXHIBIT 4.1 CURRENT STATUS OF PRESCHOOL PEDESTRIAN SAFETY EDUCATION (Cont inued)

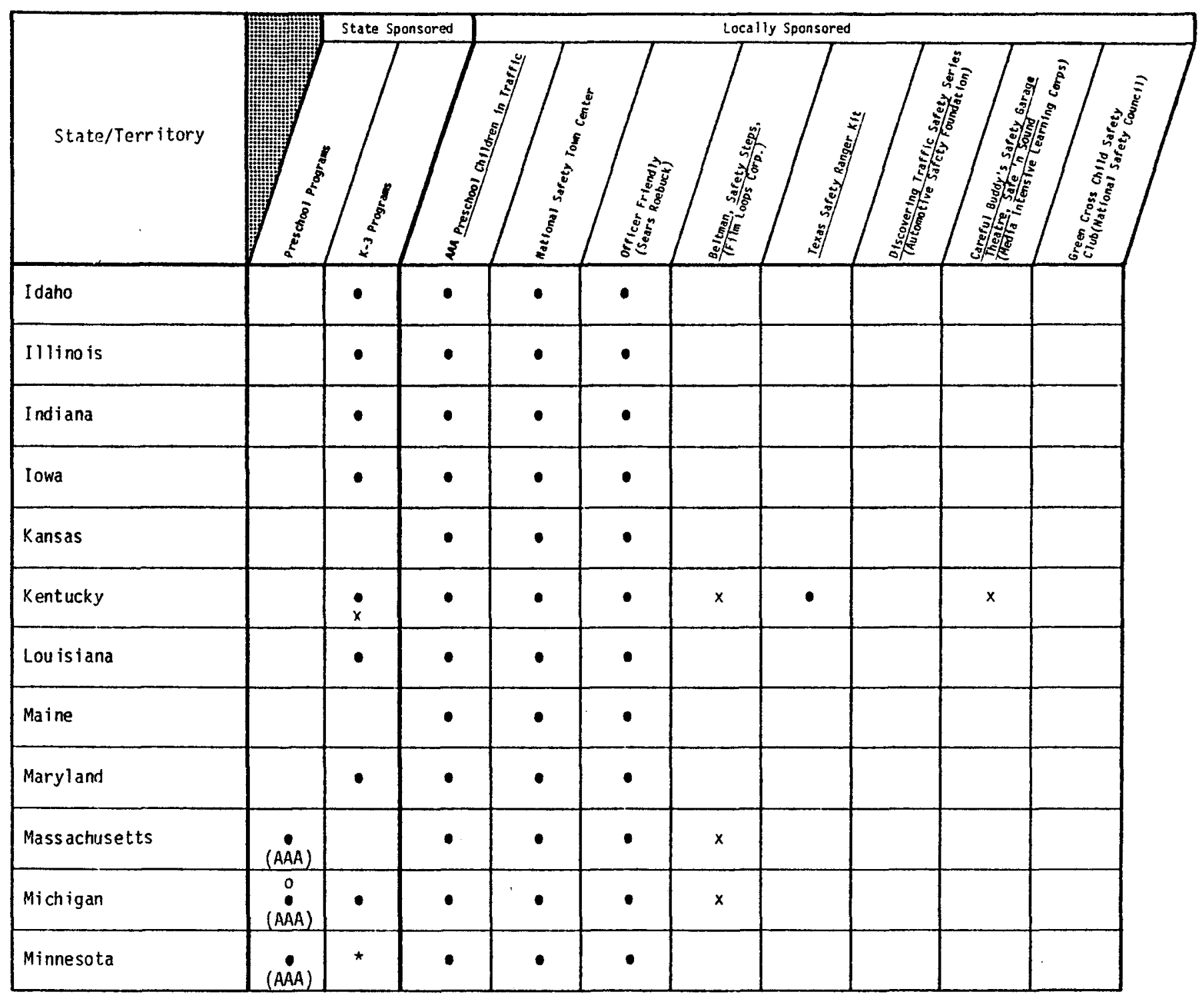


EXHIBIT 4.1 CURRENT STATUS OF PRESCHOOL PEDESTRIAN SAFETY EDUCATION (Continued)

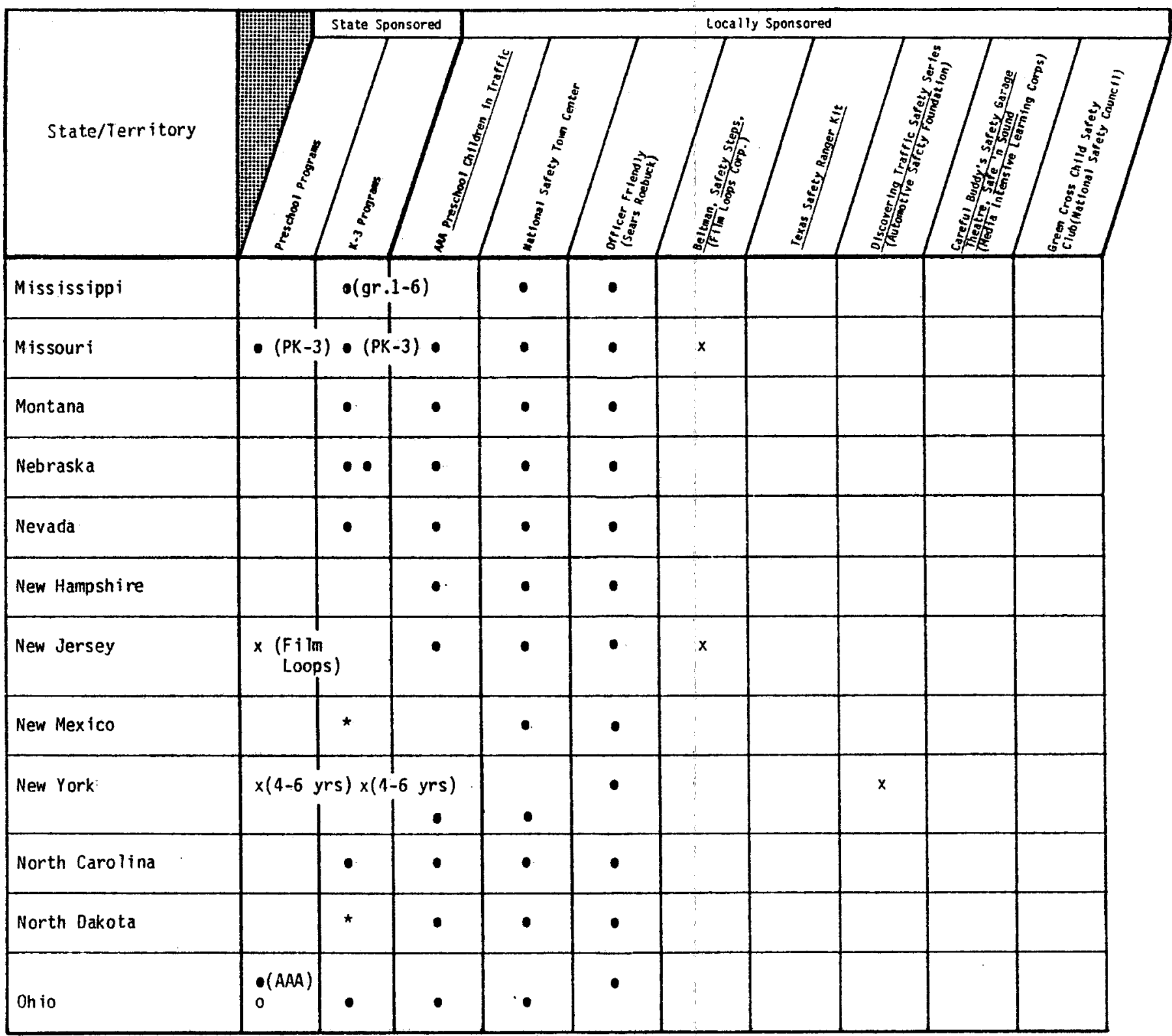


EXHIBIT 4.1 CURRENT STATUS OF PRESCHOOL PEDESTRIAN SAFETY EDUCATION (Continued)

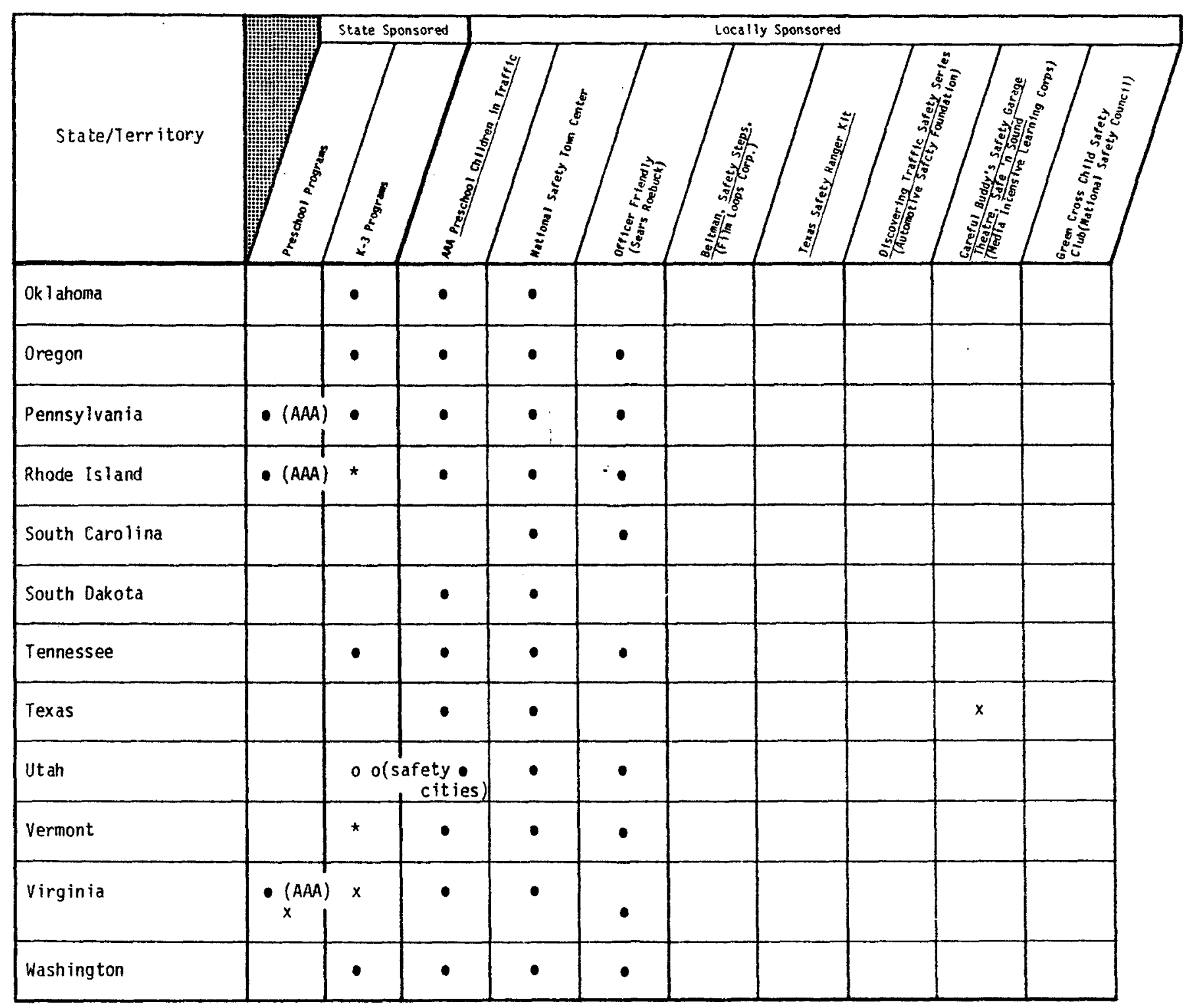


EXHIBIT 4.1 CURRENT STATUS OF PRESCHOOL PEDESTRIAN SAFETY EDUCATION (Cont inued)

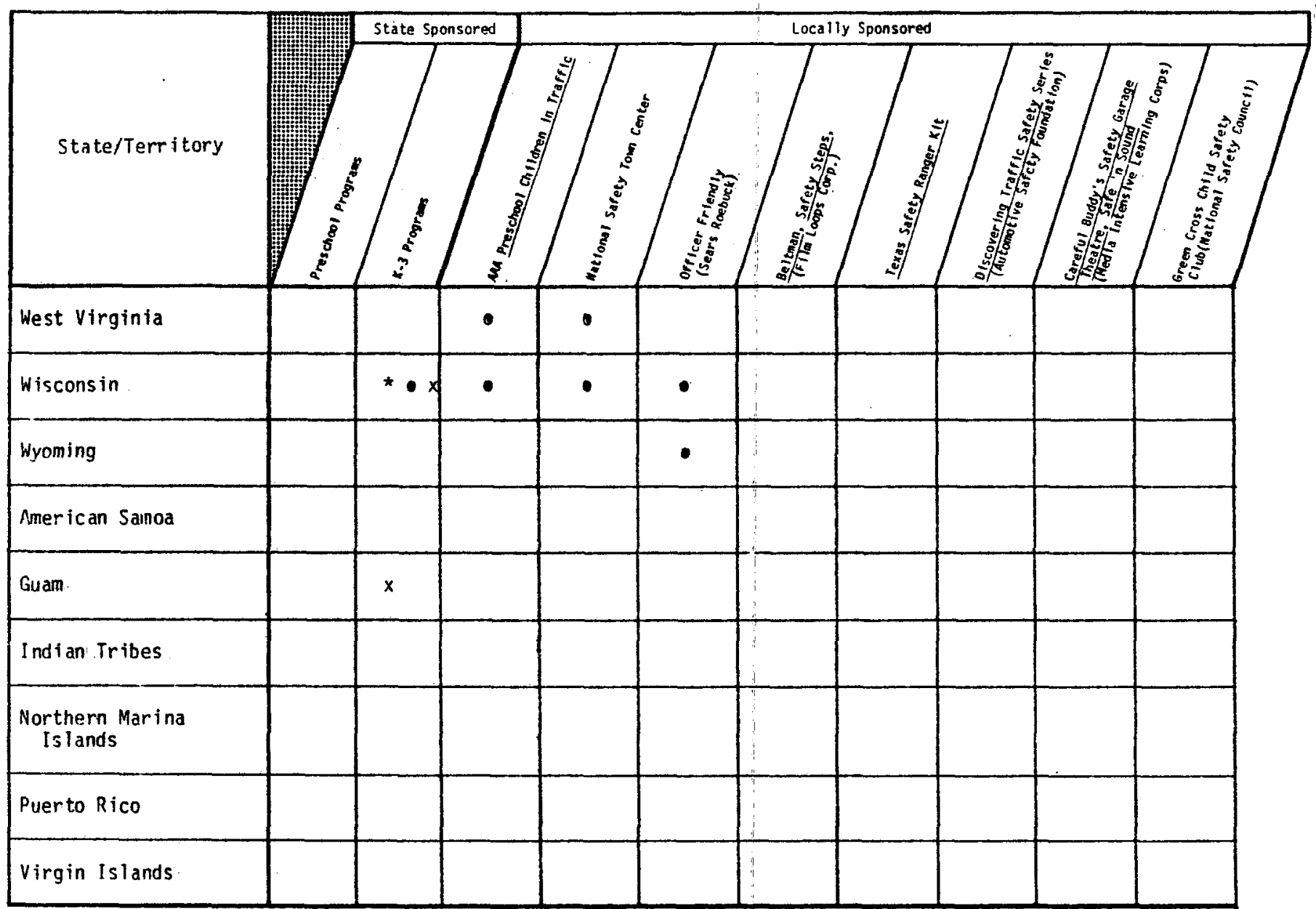

\footnotetext{
- = program

0 . = program being developed

* = coloring book/comic book

$x=$ audio-visual material

- $(A A A)=$ Preschool Children in Traffic series
}

N.B. The above information comes from the following sources: Governor's Highway Safety Representatives, AAA, and the National Safety Town Center. As the National Safety Council did not have readily available data on the states in which the Green Cross Child Safety Club was being used, the blank spaces in that column do not renresent. an ahsence nf NSr. materials heinn ussed in that ctate. 
programs. Very few of them suggest more than two or three walks outside in the real traffic environment; most of the teaching goes on inside the classroom, gym, or playground. California's recently developed curriculum and Nevada's curriculum are notable exceptions to this trend. For example, all of the lessons in the California program give children opportunities to practice safe pedestrian behaviors at roadside environments.

Program materials for most $K-3$ state curricula consist of teaching manuals which have lesson plans, dittos (mimeographic materials), lists of relevant audio-visuals and print materials, and (occasionally) additional teaching tools such as a real traffic light, posters, simulated intersection $k i t$, and accompanying audio-visuals. While program materials may be packaged in different ways, using a box of activity cards, notebook binder with loose leaf sheets or a bound teacher's manual, they generally contain lesson plans and lists of resources. Many states have borrowed ideas from other states, so that many of the same activities, dittos, and formats appear in several curricula.

The state curriculum materials of ten teach several kinds of traffic safety--bus, car passenger, bicycle, and pedestrian safety being the most common. Occasionally materials will contain units on fire prevention, school safety, playground safety and emergency preparedness. Within pedestrian safety units of ten a large number of topics will be integrated--the weather, community helpers, school safety patrol, time to leave for school, dangers of strangers as well as all the instructions on how to cross a street, etc. of course, there are exceptions. Behaviorally oriented programs, such as California's focus solely on teaching basic pedestrian skills, such as where to walk and how to cross the street.

\section{III.B The Coloring Book Approach}

Some states have developed coloring books, comic books, game books, or a combination thereof. These books are aimed at increasing children's knowledge of safety rules. Depending on the author' ' beliefs in how children learn and their attitudes towards children, these booklets tend to provide efther a humorous and entertaining or a disciplinary and admonishing approach. Some 
provide reasons for safety rules through illustrations or words while others omit this component. These booklets are, for the most part, designed to be fun for children. Most coloring books provide opportunities to color, connect the dots, or solve picture puzzles. The illustrations within are reminiscent of the dittos in the K-3 curriculum manuals. There are usually a few pictures of children walking on sidewalks, darting out into traffic, or being approached by a stranger. More of ten pictures depict police officers, signs and traffic lights. It is probably assumed that parents or teachers will read and discuss the booklets with children to clarify any misconceptions and to reinforce important points, so that in addition to teaching small motor coordination, the coloring books will increase children's knowledge of pedestrian safety.

\section{III.C. The Safety Town Approach}

The Safety Town program, $1 /$ as described by materials from the National Safety Town Center, is designed to give children many opportunities to practice exemplary traffic behaviors in a safe environment, to increase their awareness of the driver's and the bicyclist's as well as the pedestrian's viewpoints, and to learn the rules and the reasons for safe behavior. To this end, Safety Towns have been created. The safe learning environment is provided by the Safety Town, a miniature town complete with child-sized houses, signs, traffic lights, vehicles, streets, sidewalks, railroad crossing, and every representative part of a traffic environment. There children ride in miniature cars or bicycles and cross the streets under the supervision of instructors and police officers. According to the National Safety Town booklets, children in a National Safety Town Center program receive a total of three and one-half hours of pedestrian safety instruction in the Safety. Town and one-half hour of pedestrian training on a real street. In other words, they spend most of the time allotted to pedestrian safety practicing pedestrian skills in a simulated environment which, unlike the real traffic environment, affords children the visibility they would not ordinarily have: They are, however, given behavioral training, which is rare in many traffic safety programs. They receive some additional pedestrian safety instruction in classroom sessions where films, artwork, discussion, singing, and other activities are carried out. 
Pedestrian safety is only one type of safety taught in the National Safety Town Center program. School bus, bicycle, home, playground, medicine and fire safety are taught as well. The overriding belief is that children need to be taught to "think safety" in every area of their lives. It is hoped that parents, who are encouraged to attend Safety Town sessions with their children, will continue their children's safety training in the home. The Safety Town approach to pedestrian instruction which has been described above belongs to the National Safety Town Center whose program materials were available for review. There are many other Safety Town programs and, in fact, Safety cities which are currently being instituted. These programs share the same basic instructional approach utilizing a miniature traffic environment, but may use different materials and set up slightly different programs.

\section{III.D. The Officer Friendly Program Approach}

There is an officer Friendly Program sponsored by the Sears Roebuck Foundation and similar programs sponsored by local communities and/or 5 tate offices of Highway Safety. In both kinds of programs a law enforcement officer visits public elementary schools once or several times a year to promote understanding of the police officer's role in the community and to teach safety principles.

In the Officer Friendly Program sponsored by the Sears Roebuck Foundation, police officers are given training beforehand--information about $K$-third grade children and public schools, suggested activities, preferred teaching methods, and communication techniques. Within this program the following types of safety are taught: pedestrian, bicycle, bus, automobile, household, neighborhood, school, seasonal, drug, and fire. The police officer is encouraged to teach safety through such activities as role-play and discussion. He/she is encouraged to plan activities which allow children to be physically active. All activities are indoors and at no time are children given actual street crossing training or any other pedestrian training in thereal traffic environment. However, the police officer is encouraged to show slides of real traffic situations and to try to approximate the real traffic environment as closely as possible within role-play situations. 
other types of police officer programs exist and have existed for some time. One of the more famous of these was Officer Presley whose "safety dogs" performed tricks before the children and captured their attention while officer Presley reminded them of safe traffic behaviors. Officer Presley's entertainment and lecture teaching methods are a contrast with those of the officer Friendly Program. One can, however, find both styles of teaching--and others--among police officers who are visiting schools today.

\section{III.E. Children's Traffic Club Approach}

Like the children's traffic safety clubs in Scandinavia, England, and Japan, similar clubs for preschool children are being started here in the United States. NSC and the Office of the Governor's Highway Safety Representative in Connecticut are currently developing traffic clubs for preschool children.

What these efforts share in common is the club concept--the idea that children "belong" to a traffic safety organization and as members are sent materials specially developen to increase their knowledge of pedestrian safety and to improve their behavior in traffic. Because of the preschool child's developmental limitations and well-known tendency to imitate their parents' behavior, preschool children's parents take an active part in their children's membership and club activities. While children receive written materials, puzzles, games, etc., for their learning and enjoyment, parents are often sent guides to the children's materials and instruction for teaching children pedestrian safety in real traffic situations. Parents are encouraged to read the children's materials to their children and to help their children perceive the relationships between the materials and real traffic situations. Parent input is essential. Without it, children's activities might be little more than small motor, perceptual, and symbolic tasks. An additional reason that parent participation is so important is that increased and improved parental supervision and instruction are primary aims of the children's traffic clubs. There are surface similarities between the children's traffic clubs that are being developed in the United States. Both plan to send materials to children and to promote the club concept. NSC, for example, which has developed a series of safety book lets for its Green Cross Child Safety Club, sends members 
one booklet every six months. Connecticut's Preschool Traffic Safety Club intends to send out a monthly newsletter to preschool club members in that state. Both clubs also include pedestrian safety as part of a larger focus on safety in genera1. Water, home, and passenger safety are taught in addition to pedestrian safety by these clubs.

\section{III.F. The Parent Child Book Series Approach}

This approach, as used by the AAA with its Preschool Children in Traffic series, is similar to one type of club approach. Both parents and children receive materials, and parents are expected to read the books to their children and to give them on-the-street traffic instruction. There is, however, no club membership nor any sequential mailing procedures. The entire set of Preschool Children in Traffic booklets, one for parents and four for children, is sent to the home. The booklets are sequentially organized from easier to more difficult concepts, and parents are given general age and observational guidelines that enable them to decide when to introduce the next book in the series to their children. Parents are also given information about children's behavior in traffic, the kinds of traffic accidents that befall them, and general suggestions for conducting on-street pedestrian training.

Besides The Preschool Children in Traffic Series, one other series of pedestrian safety picture books for preschool children has been identified--a series developed by the Channing L. Bete Company. Like AAA's Preschool Children in Traffic series, the Bete series provides information for both parents and children. Parents are briefly told why children are vulnerable in traffic and given a list of appropriate methods for teaching children traffic safety. Unlike the AAA materials, the Bete materials for children do not follow a sequential progression. For example, the safest route to school is presented before children are taught how to cross the street. As opposed to the AAA materials, the Bete materials are humorous and entertaining in nature. Characters in the Bete fllustrations are more like cartoon characters, and drawings of the traffic environment are sketchy. Another difference is that the Bete materials list traffic rules as opposed to giving. instruction on parts of the traffic environment and ways to behave in traffic 
- Some approaches are purposely humorous and entertaining (TAPES, Will Walkright).

- Some approaches use an adult authority figure or hero to present safety rules and situations. Others use children's voices and/or child actors/actresses (Discovering Traffic Safety).

- Most audio-visual materials are designed to have follow-up discussion. In addition, Careful Buddy's Garage Theatre materials encourage teachers to set up the Careful Buddy's cardboard puppet theatre, puppets, and cassette tape so that children can act out what they learned with puppets or pantomime the story heard.

- Most audio-visual materials have supplementary teacher's guides which emphasize important concepts presented in the media presentations and suggest follow-up activities.

\section{PEDESTRIAN CONCEPTS TAUGHT TO PRESCHOOL CHILDREN}

The concepts taught are the heart of any educational program. No literature review of pedestrian safety programs for preschool children would be complete without a description of program content. The purpose of this section is to identify the concepts taught in the pedestrian programs reviewed and to present some of the questions that arose during the review of program materials.

\section{IV.A. What Should Be Taught In Preschool Pedestrian Safety Education?}

There are several answers to this question, depending on which program is being reviewed. Exhibit 4.2: A Tally of Concepts Taught in Preschool Pedestrian Programs gives a picture of the concepts that are taught and the popularity of each concept. It also shows the diversity of opinions regarding which safety concepts a preschool child needs to know.

It is interesting to note the range of concepts that have been included in some of the pedestrian safety education programs--everything from how to cross a street and the dangers of petting strange animals to roles of community helpers. Safety Action, the Idaho Traffic Safety Education Curriculum, Iraffic Safety Education for Iowa Schools K-6 Part II, Safety for Louisiana Schools, the National Safety Town Center program, and the Officer Friendly 
Information About the Traffic Environment

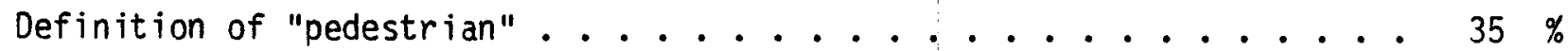

Definition of "vehicles" . . . . . . . . . . . . . . $10 \%$

Meaning of vehicle turn signals, exhaust and lights . . . . . . . $13 \%$

Definition of "street" . . . . . . . . . . . . . . 12.5\%

Definition of "sidewalk" . . . . . . . . . . . . . 12.5\%

Definition of "curb" .................. . . . . 14.5\%

Definition of "crosswalk" . . . . . . . . . . . . . . $27 \%$

Definition of "midblock" . . . . . . . . . . . . . . 4 4

Definition of "corner" . . . . . . . . . . . . . . . $6 \%$

Definition of "intersection" . . . . . . . . . . . . . $17 \%$

Definition and meaning of "traffic light" . . . . . . . . . . $83 \%$

Definition and meaning of various traffic signs . . . . . . . . . . $77 \%$

Definition and meaning of "Walk/Don't Walk" lights . . . . . . . . $42 \%$

Meaning of colors in traffic safety . . . . . . . . . . . $23 \%$

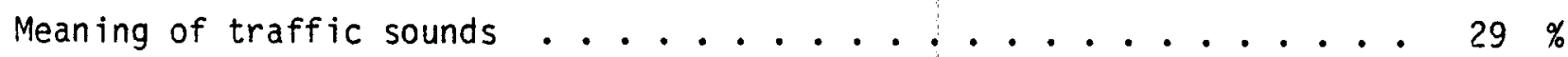

Definition of other parts of the traffic environment (e.g., alley,

driveway, overpasses) . . . . . . . . . . . . . $8 \%$

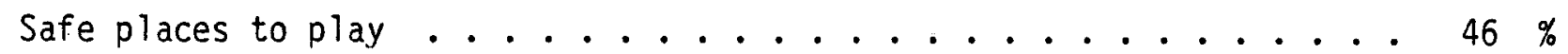

Dangerous $\mathrm{places}$ in the traffic environment (e.g., between parked

cars, railroad tracks, alleys)....................... $71 \%$

Where to walk (i.e., traffic rules) . . . . . . . . . . . $96 \%$

Safest routes (e.g., to school) . . . . . . . . . . . . $50 \%$

Commun ity/safety helpers (e.g., police) . . . . . . . . . $54 \%$ 
EXHIBIT 4.2: (Continued)

Information About Pedestrian Behavior

How to behave in traffic (e.g., scan for vehicles, listen, cross

the street) .................................. 98

Unsafe/improper pedestrian behaviors . . . . . . . . . . . . $50 \%$

Reason for pedestrian rules ................. . . $27 \%$

Ways to determine vehicle speed, distance away and stopping time . . . $13 \%$

Importance of making personal independent decisions in traffic . . . . $2 \%$

Special Problems

Being lost/personal information (e.g., name, address) . . . . . . $33 \%$

Strangers and hitchhiking ................. $52 \%$

Bad weather .................................... 48

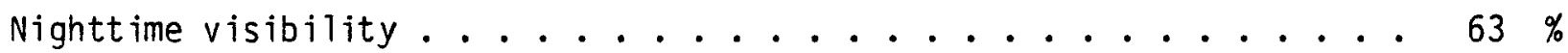

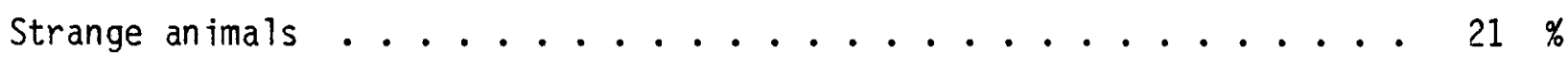

Emergencies (e.g., natural disasters, fire) . . . . . . . . . . $8 \%$

Other concepts (e.g., holiday issues, driver fallibility, I.D.P.E.,

allowing time for pedestrian trips, responsibilities to other

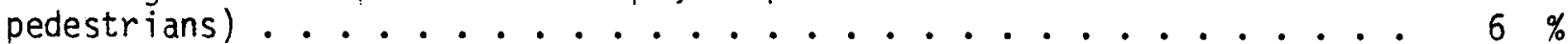

Perceptual/Motoric Tasks

Perceptual/motor tasks not directly related to traffic (e.g.,

following directions, listening to sounds) ............ $21 \%$ 
Resource Library, are examples of programs that consider it important to teach children a broad range of safety information (e.g., the wrongs of trespassing, the dangers of strangers and the dangers of deserted parking lots) as well as traffic concepts such as the meaning of traffic lights, where to cross the street and how to determine when it's safe to cross the street. In contrast, programs such as the California Guide for Pedestrian Safety Education, the Safe Street Crossing Program, and the Preschool Children in Traffic series, believe in focusing solely on teaching young children to identify parts of the traffic environment, to know where pedestrians belong and how they should act and to exhibit safe street crossing behaviors. These programs do not consider concepts such as personal information and community helpers critical to pedestrian safety education for young children.

With the exception of the Safe Street Crossing Program, which is based on NHTSA's counter measure approach, we do not know how developers of most of the programs described chose their concepts. It is not known with any certainty whether they based their programs on accident research, local demands, other curricula, or personal assessments of information young children need to know in traffic.

\section{IV.B. Which Concepts Can Preschool Children Understand?}

While the majority of state curricula designate pedestrian safety concepts

for kindergarten children (ages five to six), several programs $1 /$ do not differentiate between concepts that are appropriate for kindergarten children and those that are appropriate for children in older grades. Rarely do these programs provide guidance on the selection of concepts for age or grade leve15. One reason for this may be that program developers expect teachers to be knowledgeable about their students' capabilities in traffic, their students' abilities to understand the pedestrian concepts suggested in the

1/E.g., Alaska's K-12 Traffic Safety Education Program Delaware's Safety Action, F Tor ida's K-3 Traffic Safety Education Resource Curriculum the Missouri PK-3 Safety Curriculum Resource Guide, Ohio's Bicycle Pedestrian Safety Education: Curriculum Guide, Washington's Elementary Traffic Safety Education: A Resource Guide for Teachers $(K-6)$, Wisconsin's Project Traffic, and the Wyoming Traffic Safety Education program. 
manual, and concepts that are particularly needed and relevant for their respective age groups. Thus, teachers may be expected to determine by themselves which concepts to teach their children.

The U.S. program that provides the most guidance on concepts which are appropriate for two-, three-, four-, five- and six-year-old children is the AAA's Preschool Children in Traffic series, which has booklets for children of different age ranges. Concepts deemed developmentally appropriate for the youngest children are those which tell youngsters where to play, where to walk, how to walk on the sidewalk, and where vehicles belong. In other words, the youngest children learn about the most basic parts of the traffic environment and learn the most basic skills. Gradually children are introduced to more abstract concepts such as how to look for vehicles, what the stop sign means, and the directions cars move on two-way roads. Finally, they tackle such concepts as how to obey traffic light signals and how to scan for vehicles at intersections. In this way AAA's materials follow a sequential progression of simple to more complicated and abstract concepts.

Other programs present a sharp contrast to this developmental approach. Many programs, for instance, begin their pedestrian programs by assuming that preschool children already know basic parts of the traffic environment such as curbs, crosswalks and corners. These programs expect five-year-olds to master left-right concepts, to understand safe route to school maps, and to assess the stopping time of vehicles. Five-year-olds are of ten expected to memorize as many as 10 traffic signs and to remember what various sign shapes and colors represent. Some children are expected to measure distance by sight while others are given concrete indications by which to measure space. Sometimes they are expected to understand pedestrian instructions using negative phrasing or to understand the concept of rules.

Yet, research 1 iterature reveals that preschool children have limited capabilities in areas such as spatial relations, location of sound, visual perception, motor coordination, logical reasoning, abstract thinking, and perception of velocity. While children need to develop further capabilities in each of these areas, the literature indicates that certain concepts and skills may be too difficult for children in the preschool age group. For 
example, understanding a map of the community, showing the safest route to school, understanding concepts such as left and right, assessing vehicle speed and stopping time are, according to the findings of the literature (Piaget, Pease \& Preston, Maier, Vinje, etc.) too difficult for five-year-olds. Considering the preschool child's difficulty with abstract reasoning, expectations that preschool children identify numerous traffic signs seem inappropriate.

\section{IV.C. Which Concepts Have Safety Relevance?}

Related to the question of which concepts preschoolers can understand is the issue of which and how many concepts to teach them. Should one teach a few critical skills or should one teach many? Which skills are critical? Which are most relevant in terms of safety? Again, accident data provide some answers to the question of critical concepts. The accident data pertaining to young children show that children in the under fourteen age range are most of ten hit:

- in their own neighborhoods

- on two-way streets

- where no traffic signs are present

- when they are at play

- when they are trying to socialize

- when they are not paying attention to traffic

- in dart-out and mid-block dash accidents

- between 2 to $6 \cdot$.p.m.

- in warm weather

- on clear/cloudy days with no precipitation.

These findings raise many unanswered questions. How important is it to teach young children the safest route to school when most of their accidents occur after school and close to home? How important is it to teach preschoolers about the dangers of walking in darkness or in adverse weather when they are 
hit during daylight in clear weather conditions? Do preschoolers need to memorize all traffic signs on the road? Is it essential for a preschool program to teach children about dangers of strange animals, emergency preparedness or lime lo leave for school? Do preschool programs need to focus on intersections and traffic signal lights? Should preschool children be taught to recognize when they are playing (i.e., when they are engaged in fanta'y ur another form of $y$, hy) and when they are not playing so that the concept of safe and unsafe play areas makes sense? Do preschoolers need to be taught a special set of behaviors for what to do when they want to see a friend or relative across the strcet? These questions raise important issues which should be considered.

\section{IV.D. Are Concepts Taught in a Sequential Order?}

In many cases the answer to this question is "no." Many programs begin with extremely complicated concepts such as the safest route to school or the traffic light signal and later on teach simpler and more basic concepts such as what a sidewalk is or where pedestrians and vehicles should move in traffic. Some do not even teach those basic concepts. They skip from one topic to another-from traffic lights to community helpers to the weather, for example--with no sequence or progression of concepts whatsoever. The first lesson may be on crosswalks, the second on nighttime visibility, and the third on the dangers of crossing the street at midblock. Most coloring books, partially because of space limitations, tend to present concepts in this piecemeal fashion. Many of the state curriculum guides also prosent cuncepts in an 17 logical order. On the other hand, curricula such as Maryland's Safety Instructional System Signal Light Program, The California Guide for Pedestrian Safety Education, the Denver Pedestrian Safety Program, the Safe Street Crossing Program, and the Preschool Children in Traffic series present concepts in a recognizable sequential/developmental order. The "concepts taughl" section in each program's description at the end of this chapter will reveal the facts about each program's organization of concepts in greater detail. 


\section{PEDESTRIAN INSTRUCTION GIVEN TO PRESCHOOL CHILOREN}

Pedestrian instruction in program materials refers to statements which are used in teaching children to recognize and follow appropriate traffic behaviors. As pedestrian instruction both summarizes and pinpoints the concepts taught in pedestrian safety education programs and as this verbal instruction is used alone or in conjunction with other teaching methods in pedestrian safety education, it seems appropriate to create a special section on pedestrian instruction. This section will highlight the uses of pedestrian instruction in program materials, call attention to the pedestrian instructions given in each pedestrian program reviewed, and raise some of the issues involved in specifying instructions for pedestrian safety programs.

\section{V.A. How are Pedestrian Instructions Used?}

In the past, most curricula taught children safety concepts by one method-hearing and memorizing traffic rules. Today, although other teaching methods besides rote memorization of rules predominate, verbal pedestrian instruction continues to have a predominant place in pedestrian safety education programs. A few current program materials (e.g., several coloring books and media presentations) primarily consist of one pedestrian instruction after another. Other program materials such as the state curriculum materials, the Safe Street Crossing Program, and the AAA's Children in Traffic series give pedestrian instruction in the context of actual on-street traffic training, role-play, and other teaching activities. Within program materials which use such diverse teaching methods, a list of pedestrian instructions helps to summarize the concepts taught. Yet some program materials (such as many of the state curriculum materials) present many pedestrian safety concepts but do not. state pedestrian instructions directly or highlight them in a separate section. One has to read through these materials and piece together for oneself the pedestrian instructions to be taught to the children.

Stating pedestrian instructions in program materials has many benefits. For example, pedestrian instructions clarify the concepts presented and provide teachers and students with an agreed-upon standard of behavior. Clearly stated instructions enable a teacher to be more precise and consistent in instruction. 


\section{V.B. Which Pedestrian Instructions Are Taught?}

Each program described at the end of this chapter contains a separate section on pedestrian instructions. This section has been included because it provides a more detailed account of the concepts taught in a pedestrian program. The pedestrian instructions are, in a sense, critical elements of any pedestrian safety program.

\section{V.C. Do Pedestrian Instructions Vary Across Programs?}

A review of pedestrian instructions described at the end of this chapter reveals significant differences on a few of the pedestrian instructions. Differences on the following issues have been identified:

- Directions to scan for traffic. Some program materials teach children to look left-right-left for approaching vehicles while others teach looking left and right, and one teaches looking right-left-right.

- Where to cross the street. Most materials advocate crossing only at the corner and never entering the street at mid-biock. However, several programs instruct children to cross at crosswalks wherever they are located in addition to crossing at corners. Only a few programs give children instructions on entering and/or crossing the street at mid-block. These programs include the Denver Pedestrian Safety Program, the Safe Street Crossing Program, and the AAA's Preschool Children in Traffic series.

- The ball-in-the-street situation. In light of the disagreement on the mid-block crossing, it is not surprising that pedestrian instructions applying to the ball-rolling-into-the-street situation vary. Some instructions state never to run into the street to get a bal1, but to stop, look for vehicles, and retrieve the ball if no vehicles are coming. A few programs tell children to ask an adult to get the ball rather than get it themselves.

- When it is safe to cross the street. Another area of disagreement centers on how to teach children when it is safe to cross the street. Children may be taught that it is safe to cross the street when an approaching car is one or more blocks away. Some children may be expected to base a decision to cross on the speed and distance of the approaching vehicle. In most cases, however, children are told to cross when there are no vehicles coming. 


\section{V.D. How Explicit Should Pedestrian Instructions Be?}

The pedestrian instructions given in pedestrian safety program materials vary in terms of how detailed or explicit they are. For example, sometimes children are merely told to play in "safe" areas. However, these areas are not further defined. Sometimes children are cautioned to play as far away from streets and sidewalks as possible. Sometimes they are warned not to play in the street, on railroad tracks, in alleys or in parking lots but are not given specifications regarding permissible play areas. Then again, some pedestrian instructions state both that children should play in yards or playgrounds and not in the street. Sometimes a pedestrian instruction is so vaguely stated that learning it could make chlldren more vulnerable in traffic situations. For example, many instructions (especially those in coloring books, songs, and chants) tell children to look in different directions but do not tell them to watch for approaching vehicles.

\section{V.E. How Should Pedestrian Instructions Be Phrased?}

Both psychologists (e.g., Piaget, Inhelder, Smith) and traffic safety researchers (e.g., Sande1s, Cattell and Lewis, Sargent and Sheppard) agree that the vocabulary of the preschool child is limited. Words such as "pedestrian," "crossing," "traffic island," "passenger, "traffic rules," "left," "right," "ahead," "vehicles," and "one block' away" are examples of language that is frequently misunderstood by young children. Many such words are used in pedestrian instructions listed in the program materials reviewed. Yet the issue of language was addressed in few of the pedestrian safety program materials that are directed at parents or teachers of young children. For example, K-3 curriculum guides rarely provided guidance about appropriate phrasing of pedestrian instructions. Only materials written expressly for children, such as coloring books, and children's pedestrian safety books, provided such guidance to the adult.

\section{V.F. Should Pedestrian Instructions Consist of Do's and Don't's?}

Intimately related to the discussion of language used in pedestrian instructions is the issue of whether preschool children should be given 
instructions that use negation in their phrasing. Most children receiving pedestrian safety instruction suffer through long lists of "Don't's." "Don't play in the street." "Don't you dare go across that street." "Don't run." Several K-3 curriculum materials and coloring books present a list of "Don't's." Others state directions in positive terms, for example, stating only "Walk across the street" rather than adding a "Don't run" clarification or stating only "Don't run." At issue here is whether preschool children have the ability to comprehend negation. The literature as described in chapter 2 (page 2.52) indicates that many preschool children do have difficulty understanding "Don't" and "Not" statements. Since preschool children are just beginning to understand these concepts, it would seem appropriate to minimize the use of negation, particularly more difficult forms (i.e., "not $x$ " statements) in pedestrian safety instructions and to state these instructions in a positive manner.

\section{TEACHING METHODS USED IN PEDESTRIAN PROGRAMS FOR PRESCHOOL CHILDREN}

As relevant and appropriate as the concepts and pedestrian instructions in any pedestrian program may be, none will be effective if the methods for teaching such ideas and skills are inappropriate for the learner. This fact applies particularly to programs for the preschool age child whose thinking processes, psychological, emotional, and physical makeup are, as previously described in Chapter 2, qualitatively different from those of an adult. This section of the Literature Review will discuss the teaching methods currently being used in pedestrian safety programs for preschool children and will discuss some of the issues involved in selecting teaching methods for that age child.

\section{VI.A. Which Teaching Methods Are Used?}

This review of program materials used in pedestrian safety programs for preschool children has revealed that preschool children are being taught pedestrian safety through diverse methods and combinations of methods. Children are taught pedestrian safety through discussions and are expected to memorize traffic rules. They are read fairy tales such as "Jack and Jill" and stories about real children in traffic and their traffic environment. They 
are shown films about imaginary creatures and superhuman heroes who rescue ignorant pedestrians from danger and then recite and give reasons for traffic rules. Children are also shown films of real children coping with real traffic situations and modeling appropriate pedestrian behaviors. Children listen to tapes and try to identify traffic sounds. They are given coloring books and dittos (mimeographic materials) to color, safety puzzles to fit together, and art materials with which to create models of traffic lights, traffic signs and whole traffic environments. They do experiments such as seeing which colors are visible in a darkened room and watching what happens when an egg falls from a sheif to the floor. Children do exercises, run obstacle courses and play games to increase their understanding of directions, their perceptual capabilities and their ability to move their bodies in space. They pretend to cross an imaginary street and look for imaginary cars in the classroom. They practice stopping at the curb, scanning for traffic, and crossing the street in a simulated child-sized traffic environment such as Safety Town. They go for walks outside to identify real sidewalks, curbs and signs, and to approximate how far away cars are and how quickly they will be able to stop. Some children practice crossing real streets within a mass of children. Others practice crossing individually or with an adult. All these teaching methods--and more--can be found in pedestrian safety programs for preschool children in the United States. Exhibit 4.3: "Prevalent Teaching Methods Used in Pedestrian Programs for Preschool Children" presents the teaching methods used in the 51 U.S. traffic safety programs reviewed for this Literature Review.

\section{VI.B. What Commitment Is Made Toward Traffic Training?}

As can be seen from the chart, many programs recommend teaching children pedestrian safety in the real traffic environment but few programs have made a strong comitment to this teaching method. Some programs only take children outdoors a few times a year--to identify signs, to look at traffic lights, and to scan for cars, for example. These school programs are primarily indoor classroom programs which rely upon audio-visuals, dittos, role-play in a simulated traffic environment, bulletin board displays, classroom discussions, and games to teach pedestrian safety. Other programs do not include any 


\section{EXHIBIT 4.3: PREVALENT TEACHING METHODS USED IN PEDESTRIAN PROGRAMS FOR PRESCHOOL CHILDREN}

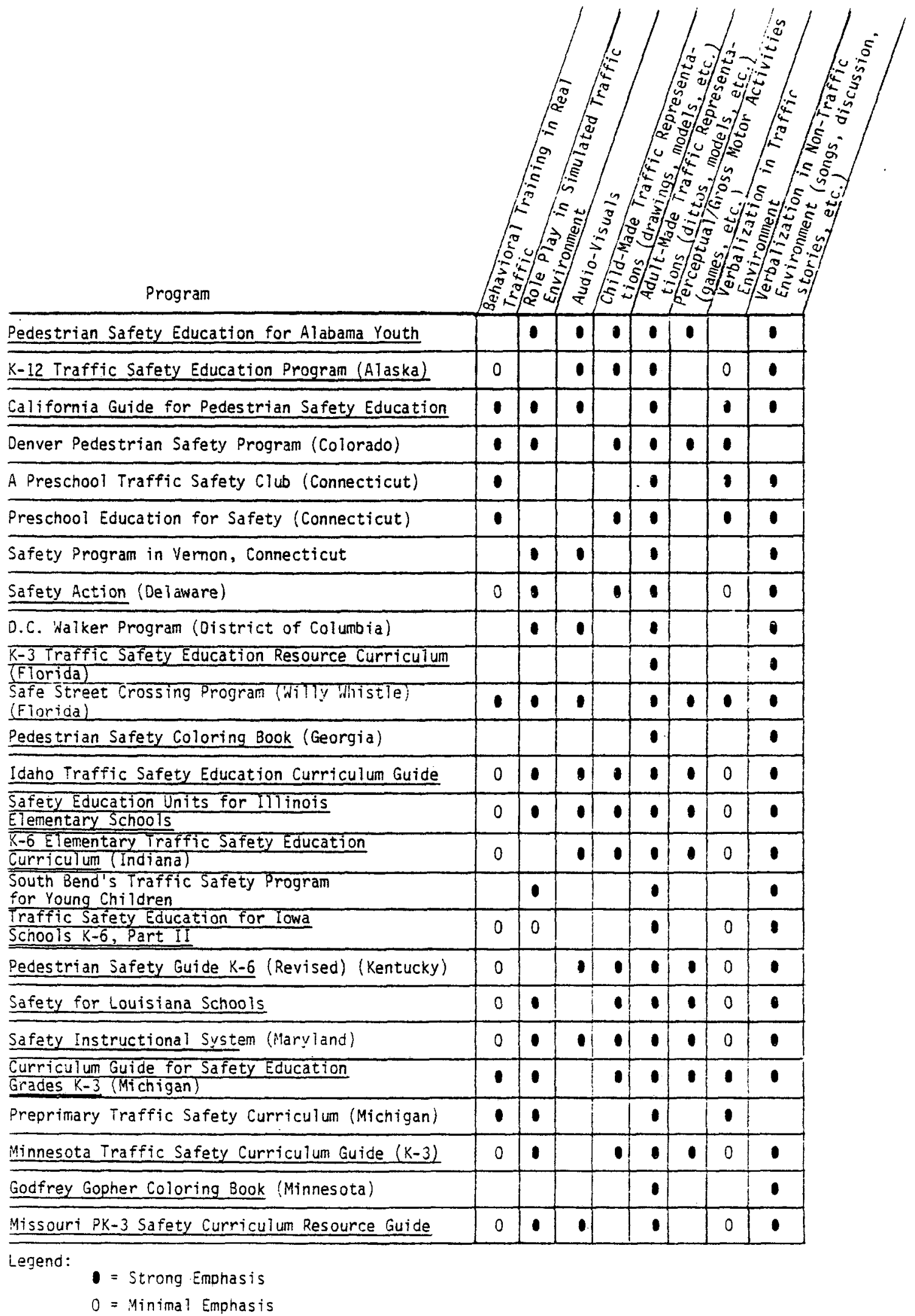


EXHIBIT 4.3: (Continued)

Program

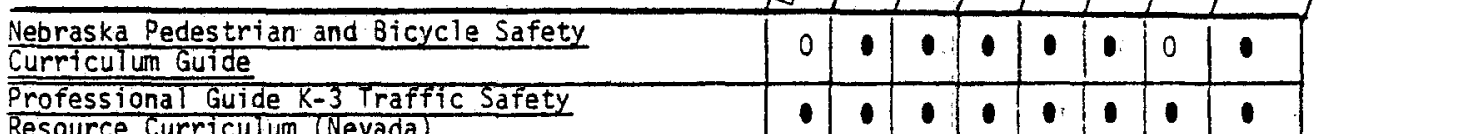

Resource Curriculum (Nevada)

Traffic and Fedestrian Education Systems

(New York

K-9 Traffic Safety Resource Curriculum

(North Carolina)

The Governor's Highway Safety Coloring Book

(North Dakota)

Bicycle/Pedestrian Safety Education:

Curriculum Guide (ohio)

Ok lahoma Curriculum Guide and Student Handbook for

Teaching Pedestrian Safety Education K-3

Traffic Safety Education for Oregon Schools $(K-3)$

A Teaching Guide for Child Pedestrian Traffic Safety (Pennsylvania)

Color Your Streets Safe (Rhode Is land)

Elementary Safety Education for Utah Schools

Safety Forever: A Comic Book About Safety (Vermont)

Will Walkwright programs (Virginia)

A Teacher's Guide for the Primary Level Traffic

Safety Education $k i t$ K-3 (Washington

ETementary Traffic Safety Education:

A Resource Guide for Teachers $K-6$ (Washington)

Project Impact (Wisconsin)

My Safety Coloring Book (and Teacher's Guide)

(Wisconsin)

Project Traffic (Wisconsin)

Wyoming Traffic Safety Education

Preschool Children in Traffic (AAA)

Green Cross Child Safety Club (NSE)

Channing L. Bete Co. Eraffic safety education materials

Safety Town (National Safety Town Center)

Officer Friendly Resource Library (Sears Roebuck)

Officer Friendly Teacher's Idea Book (Hartford

Police Dept. and Board of Education

Aetna K-2 Pedestrian Safety Program

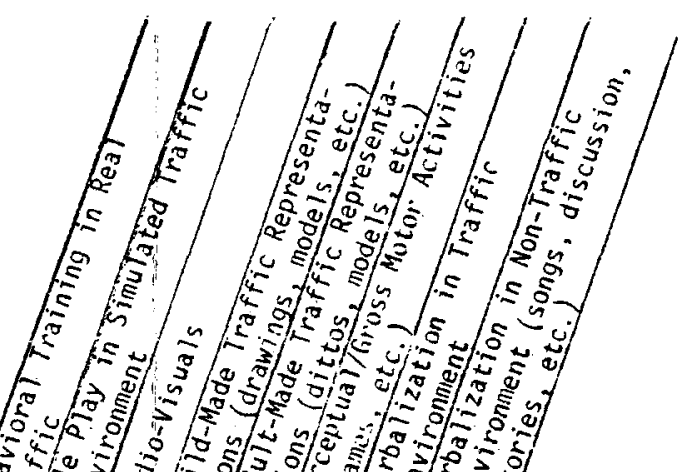


outdoor training in their programs. These programs include the officer Friendly Program, the Aetna K-2 Pedestrian Safety Program, the Will Walkwright program, the D.C. Walker prourall, Florida's $\underline{K}-3$ Iraffic Safety Education Resource Curriculum, and the Pedestrian Safety Education for Alabama Youth program.

In contrast, the following school-based programs strongly espouse on-strcet pedestrian training: the Californta Guide for Pedestrian Safety Education, the Denver Pedestrian Safety Program, Michigan's Curriculum Guide for Safety Education $(K-6)$, Nevada's Professional Guide $(K-3)$ Traffic Safety Resource Curriculum, and the Safe Street Crossing Program in Dade County, Florida. Developers of both Michigan's Preprimary Traffic Safety Curriculum and Connecticut's Preschool Education for Safety programs have indicated strong beliefs in on-street training. For safety reasons, some of these programs carry out some of their training sessions next to and in blocked off streets, driveways, or parts of playgrounds which are made to resemble the real traffic environment as much as possible. All these programs believe strongly that behavioral training in real traffic situations is critical in order to produce behavioral changes.

The pedestrian programs which are home-based and use parents or caretakers as teachers of traffic safety also voice a strong commitment to training in the real traffic enviroment. Such programs include AAA's Preschool Children in Traffic program, NSC's Green Cross Child Safety Club, and thr. Chaming : Bete book series. Developers of Connecticut's Preschool Traffic Safety Club also plan to stress training in real trafic. 
Programs that do extensive outdoor on-street training of children appear to have developed detalled pedestrian instructions for young children. This is one of their important contributions.

VI.C. Is There A Place For Active Learning and Discovery In Pedestrian Safety Education?

Many early childhood educators (e.g., Froebel, Montessori, Dewey, Bruner) and researchers (e.g., Piaget, Flavell) have advocated providing preschool children with opportunities to learn through action and discovery. It is interesting to note the ways in which several pedestrian programs have incorporated principles of active learning and discovery into their teaching methods.

Many programs recognize that young children need to be physically active. Children are given dittos and puzzles and role-play situations and games to keep them physically moving. Physical activity, however, may not in and of itself generate active learning. I/ It is possible, for instance, that a child coloring a picture is gaining small motor skills but no knowledge or skills in pedestrian safety. The kind of active learning which early childhood educators refer to is the kind of learning in which a child is making observations, drawing conclusions, and making decisions. Because of the preschool child's need for concrete materials and sensory experiences, active learning also connotes physical activity for the preschool child.

Several pedestrian programs have incorporated the concept of active learning as described above into their programs. For example, in the Preschool Children in Traffic series, parents model and explain correct pedestrian behaviors in the real traffic environment and then ask children questions to encourage them to make observations of vehicles and parts of the traffic environment. The Safe Street crossing Program sets up situations in which children are actively involved in making decisions such as to how to enter the street to get a ball. Most programs which give children individualized on-street training experiences provide children with active learning experiences.

1/As defined by Hohmann, Banet and Weikart, 1979; Bruner, 1962; Taba and Elzey, 1964. 
Learning through discovery is another matter. Children cannot be allowed to learn through discovery if that involves letting them walk dangerously into the street and learn the consequences of unsafe actions through an accident. They can, however, discover through observation. They can discover that traffic lights differ, that some streets are one-way streets and that the class of vehicles includes cars, trucks, bicycles, etc. The Automotive Safety Foundation's film series, Discovering Traffic Safety has, as its name implies, incorporated the principle of discovery into its learning materials. Each film presents children with a real traffic situation which children are encouraged to analyze. From their analyses they "discover" certain principles of traffic safety. Such an approach used in real traffic training is an example of one way that children can be allowed to discover important concepts in pedestrian safety.

\section{VI.D. What Types of Materials Are Designed for Children?}

The types of materials designed for children include storybooks, dittos, posters, puzzles, paper and pencil games, siides, films, and coloring books. The children's materials reviewed in this volume include all of the above. They were examined with the following questions in mind:

- What types of traffic situations are presented?

- What types of geographic areas are shown?

- How realistic/how abstract are the traffic environments?

- Do the traffic environments and characters depicted look current?

- Are children depicted? Adults? Other figures?

- Is there adequate representation of different social, racial, and ethnic groups?

- Are both sexes equally represented in traffic situations?

- How much language is on each page? Is the length appropriate for preschool children? Are sufficient or too many concepts presented per page?

- Which kinds of words are used? Would a preschool child understand them? 
- Are new words defined for children? If so, are they defined in terms of other words or fllustrations?

These questions pertaining to children's materials are answered in detail in each program summary.

Generally speaking, dittos (mimeographic materials) in many state curricula vary in terms of level of abstraction. A few dittos have diagrams of intersections that look like the same diagrams used in a driver's education course. These are some of the most abstract materials seen in any manual. Signs are less abstractly depicted, but they rarely have posts or any environmental context. In some cases, city blocks--a concept used in helping children judge car distance--are depicted as geometric cubes (they bear not even a vague resemblance to real city blocks.) On the other end of the scale, a few dittos are somewhat realistic, showing, for example, two children crossing a very recognizable street with sidewalks, grass, bushes and other details which enable the young child to understand the representation. A problem with most dittos is that they do not present enough examples of the traffic environment and perestrian situations. Few scenes from urban traffic environments are depicted. Often children are shown in no traffic context. For example, the ditto focusing on wearing bright clothes in bad weather usually presents a few children wearing raincoats and no traffic environment. Illustrators of ten fail to show a car approaching children who are crossing an intersection in the rain; an opportunity to show why bright clothes are needed is lost. A few dittos do not even present scenes that relate to traffic situations. Pictures of Humpty Dumpty, Jack and 3111, and The 01d Woman Who Lived in a Shoe are presented in several curriculum sections on safe and unsafe places to play. Such dittos expect preschool children to transfer a partially demonstrated idea in a fairy tale (i.e., safe and unsafe places to play) to real traffic situations. These illustrations, along with the diagrams of intersections, are examples of dittos which would be the most difficult for preschool children to understand.

There are a few examples of curriculum dittos which depict traffic environments with realism and characters from different racial, ethnic, and socioeconomic backgrounds. The more recently developed curricula are more 
likely to have incorporated minorities, up-to-date, clothing and people from different socioeconomic backgrounds in their materials. The majority of state curricula, though, are lacking in this area. Only one or two curricula such as Florida's K-3 Traffic Safety Education Resource Curriculum contain dittos that present a variety of traffic environments with great detail and realism.

Language is rarely present on dittos. Most dittos which have words include them as directions.

In addition to dittos, illustrations in children's books were reviewed. Two of the book series reviewed, the AAA's Preschool children in Traffic series and NSC's My Safety Book from the Green Cross Child Safety Club, contained realistic pictures with traffic environments fully illustrated. Both series depict persons from minority and caucasian groups. Both series depict scenes from middle-class suburban types of neighborhoods, some of which could conceivably represent city neighborhoods on the outskirts of the city. No booklets illustrating preschool pedestrian situations in clearly recognizable rural or inner city traffic environments have been identified. In contrast to the AAA and NSC materials just mentioned, the Channing $L$. Bete series contains humorous cartoonlike drawings of people in traffic. All the Bete characters are white and middle-class and appear to belong to the 1950s era. The drawings of the traffic environment are very sketchy.

\section{VI.E. Which Teaching Methods are Appropriate for Preschool Children?}

Which teaching methods affect both knowledge gains and behavioral changes? One of the crucial questions program developers must ask themselves when designing a pedestrian program is, Which teaching methods will increase both preschool children's knowledge and pedestrian safety skills and reduce their traffic accidents?

Research in the field of traffic safety education has provided some answers to these questions. In general, European research supports the practice of behavioral traffic training in real traffic situations. Sandels $(1968)$, citing the Solna study, reports that interview and traffic test 
measures indicated that children achieved the best results from combined outdoor and indoor training and the poorest results from indoor training alone. Children gained more theoretical knowledge from indoor teaching than from no teaching at all, however. (Outdoor training was conducted on a real street, barricaded without the children's knowledge.) Research conducted by Nummenmaa, Ruuhilehto and Syvanen (1975) concurs with results of the Solna Study: Nummenmaa et a1. (1975) found that children's traffic behavior showed greater improvement when traffic instruction included both real traffic and film instruction than when only filmed instruction was given. Schioldborg's (1974) study showed that increasing children's knowledge does not necessarily improve their behavior. According to Schioldborg's study (1974), although 80 percent of the children in the study knew that they should look for vehicles before crossing streets, only 34 percent actually did so. Pease \& Preston's (1967) study showed that teaching children songs and maxims about traffic safety was virtualiy ineffective. Like Nummenmaa (1975) and Sandels (1968), Schioldborg (1974) found that training on real roads combined with training in classrooms was a very effective teaching method. Sightly less effective was training on real roads without classroom training. In other words, the further the training deviated from real traffic training the less effective it became. However, the combination of real traffic training and classroom training was the most powerful combination of all:

How could one explain the above findings? One way to interpret such findings is to look at the teaching methods used in terms of the types of learning experiences they provided for the child. The training on real roads provided active and concrete learning experiences for the child--experiences which helped the child to form internal images of parts of the traffic environment and of sequences of behaviors expected from pedestrians and vehicles. The experiences in the classroom forced the child to think more abstractly, to envision in his/her own mind all the objects and events experienced firsthand. Research by Piaget (1958) lends credence to the assumption that children need the real experiences first, followed by opportunities to physically practice the behavior and to re-invent it through symbolic means (e.g., language, role-play, etc.). 
Looking at the various teaching methods used in the pedestrian safety programs for preschool children, one can classify them according to types of learning experiences provided for the child. One can ascertain which teaching methods provide children with opportunities to gain concrete firsthand experiences and which encourage them to imagine, re-invent, or think abstractly. Exhibit 4.4 classifies the teaching methods used in the pedestrian programs reviewed in terms of the types of learning experiences they provide and their levels of abstraction. By matching such an instrument to the list of teaching methods provided in the pedestrian programs reviewed, it is possible to ascertain the kinds of learning experiences they are providing for young children and to assess to some degree their promise of effectiveness.

Based on this Literature Review, we have identified two essential components of a pedestrian safety program for preschool children--a knowledge component and a behavior component. The first refers to educational experiences designed to give children an understanding of the traffic environment. The second component refers to behavioral training in correct pedestrian procedures.

\section{VI.F. Teaching Knowledge}

This section on knowledge-oriented components of pedestrian programs focuses on three major issues: (1) the ways in which young children learn about the traffic environment; (2) teaching methods that best meet children's needs for first-hand learning experiences; and (3) teaching methods that best respond to children's needs to recall and synthesize their direct learning experiences. Discussion on each of these issues follows.

According to research studies cited in Chapters 1 and 2 of this report, two of the reasons that preschool children have difficulty coping with traffic are that they have an incomplete knowledge of the traffic environment and that they tend to focus on and pay attention to only one or two parts of the environment at one time. Often the parts of the traffic environment with which preschool children are familiar are those parts which catch their eye or have special meaning to them. The ambulance, cars, other children, the ice 
Levels of Abstraction Kinds of Experiences Teaching Method

High

Re-inventing or reviewing experiences through words (which bear no resemblance to the real object, person, or event)

Highly Symbolic Teaching Methods

- Discussion

- Lecture

- Songs

- Recitation of rules

- Oral stories

- Written word

Re-inventing or reenacting experiences

Symbolic Teaching Methods through pantomime or through use of physical props (which bear some resemblance to the real object, person, or event experienced)

Low

First hand concrete learning experiences

Concrete Teaching Methods

- Behavioral training in the real traffic environment 
cream truck, the walk light signal with the button--these may be some of the parts of the traffic environment which are especially significant to preschool children. In other words, these may be the parts of the environment on which preschool children focus to the exclusion of everything else.

How can we as educators help preschool children develop a more complete awareness of the traffic environment around them? How can we increase their knowledge? It is clear that child development research indicates that young children learn through sensory input--through hearing, smelling, watching, tasting, and acting. They learn through firsthand experience. It is probable that young children learn about the "walk" light signal because they push the button. Their interest in and knowledge of cars is most likely linked to the fact that they can physically get inside cars, hear the engine sounds, and move the steering wheel. They may be aware of ambulances because of the shocking sounds of their sirens. How can we help children to pay attention to other, perhaps less exciting parts of the traffic environment such as sidewalks, curbs, crosswalks, and parked cars? The answer to this question is very complex since even if children know about all parts of the traffic environment, they may only attend to certain elements because of their impulsive, egocentric, and emotional nature, and the nature of their thought processes. However, we believe that a combination of increased traffic awareness and traffic training may help children to be more cognizant of that environment.

Drawing upon the results of developmental research and European research on pedestrian programs (previously mentioned), we have concluded that the most effective way to teach children about the traffic environment is to enable them to experience it directly through all their senses and then to represent or express what they have learned through a variety of media such as language, role-play, movement or art. 1/ For example, children first need repeated direct experiences walking on sidewalks, looking at real signs, and watching for vehicles so that they can establish images of all these actions and parts of the environment in their minds. Next they need opportunities to recreate

1/The concept of representation is explained in Hohmann, Banet, and Weikart, 1979; Piaget, 1962. 
or remember what they have experienced. Children benefit from opportunities to construct streets and sidewalks with building blocks, draw pictures of sidewalks and crosswalks and pretend to stop at a curb. Not only do such representational experiences challenge children to remember separate parts of the traffic environment, but they also encourage children to remember how parts of the environment are related. Orawing a picture of the child's experience in crossing a street forces the child to recall where the sidewalk was in relation to $\mathrm{him} / \mathrm{her}$, where one sidewalk was in relation to the other, and where cars were in relation to him/her.

Many existing pedestrian programs provide children with firsthand experiences in the real traffic environment. As far as firsthand experiences are concerned, the majority of pedestrian programs suggest at least one or two walks outside in the real traffic environment. Unifortunately, few programs base their teaching on direct experiences. Children in most programs are not given repeated opportunities to experience the real traffic environment. It is assumed that after a few lessons about parked cars that they will start to notice parked cars. This may, in fact, happen. However, research has shown that children usually need repeated experiences with an object in order to understand it.

Many pedestrian programs provide children with representational experiences. There are two types of representational experiences children can have: one type gives children opportunities to create or represent and the other requires children to interpret those representations which others (usually adults) have created.

Programs which encourage children to represent their own experiences provide children with opportunities to role-play, draw, make models, and discuss firsthand experiences. Many experience-based programs suggest asking children questions about what they notice in the traffic environment while they are there. This teaching method is very appropriate for preschool children. It is easier for them to discuss (to represent) the traffic environment when it is present than it is to discuss it in the classroom. Another technique programs favor is asking children to role-play pedestrians in various traffic situations. Once again, the further children are from the 
real environment, the more difficult and abstract the task becomes. That is why several programs have gone to great pains to create simulated traffic environments which bear close resemblance to the real environment. A third representational experience children are given is artwork. One problem with many existing pedestrian programs is that they only require children to represent parts of the traffic environment--a sign or a traffic light--rather than the whole. Several programs also give children models to copy. For example, children try to make signs or draw streets exactly like the teacher's. This deprives children of the true representational experience and deprives the teacher of the opportunity to learn what the children have learned about the traffic environment. (Children represent what they remember, not everything they have seen.)

In summary, children in many existing pedestrian programs are given opportunities to remember and recall their firsthand experiences in traffic environments. However, most pedestrian programs which make use of these opportunities have not made teachers conscious of the representational process nor have they encouraged teachers to be systematic in relating firsthand experiences to representational experiences.

Almost all pedestrian programs give children opportunities to interpret drawings, films, photographs and other representations of the traffic environment. In fact, programs of ten provide numerous adult-created representations for children to interpret but provide few opportunities for children to represent on their own. Adult-created representations of the traffic environment can be used to help children focus on one particular part of the environment or the whole. The experience of interpreting another person's picture of traffic situations and environments is a valuable one, as it encourages recall of the real environment.

In the estimation of the reviewers, the most appropriate adult representations of the traffic environment for children should be highly realistic. As preschool children are just starting to master the use of symbol systems, the less symbolic and abstract a drawing or a film is, the more meaning it will have to the preschool child. Some of the most exciting films, photographs, and drawings show the traffic environment from the child's 
height and view point, thereby presenting children with scenes with which they are most familiar. Exemplary illustrations present parts of the traffic environment such as traffic signs and crosswalks in full traffic context. In contrast to dittos with big hexagonal shapes and the symbols S-T-0-P covering an entire page, exemplary illustrations depict stop signs rooted in the ground and vehicles stopped beside them. In contrast to pictures of children in rain gear or children in clothes that should be colored light colors, exemplary lllustrations teach children about the need for visible clothing in bad weather and darkness by showing rain beating down between vehicles and pedestrians, and darkness hiding dark objects from the driver's view. Exemplary illustrations show recognizable urban, suburban, and rural scenes, pedestrians of different ages, sexes, races, and ethnic backgrounds, and vehicles of all kinds. The more realistic and representative of varied traffic environments and pedestrians, the more meaningful and instructive adult representations will be to the preschool child.

\section{VI.G. Teaching Appropr iate Behavior}

Like the previous section discussed the need for both repeated firsthand and representational experiences in knowledge components of pedestrian safety programs, this section advocates the same approach in the teaching of pedestrian behavior. In addition, it discusses children's need for a training sequence which teaches a sequentially developed series of specific concrete behaviors as well as their need for positive models. Ideas about ways to best utilize models in a pedestrian program and ways to increase the effectiveness of parent models are also explored.

Just as children need firsthand experiences to gain knowledge of the traffic environment, they also need firsthand learning experience practicing correct pedestrian behaviors in the real traffic environment. Behavioral research advocates teaching behavior in a systematic step-by-step fashion, from simple to complex behaviors. Various researchers and program developers have applied task analysis techniques when analyzing pedestrian procedures and have suggested a series of behaviors which children need to learn in order to master such skills as crossing a street and crossing a controlled intersection. For example, they teach children (1) to walk on the sidewalk: 
(2) to stop when they come to a curb; (3) to look to one side for vehicles; (4) to look to the other side for approaching vehicles; (5) to recheck the first direction for moving vehicles; $(6)$ to follow the path of any moving vehicle with their eyes (and with their fingers) and to stand and wait and scan until no cars are coming; and, (7) to walk straight across the street only when no vehicles are coming. This kind of behavioral training stands in contrast to the early teaching of pedestrian safety in which children were merely told a traffic rule and expected to automatically know all the steps involved in a pedestrian task such as crossing the street. Programs which advocate step-by-step on-street pedestrian training stress the importance of repetition using the same step series, the importance of role models, and the need for children to keep alert to traffic while they perform the series of steps. Trying to prevent behavioral training from becoming a ritual, many educators encourage children to explain what they are doing and why when carrying out pedestrian tasks such as stopping at the curb. Interpreting these educators' strategies in another light, one might say that they are asking children to represent their actions with words. In fact, the teaching method of encouraging children to experience traffic and represent their experience applies as much to behavioral learning as it does to learning about the traffic environment.

Many programs have utilized role models to teach children traffic safety behaviors. Parents, school safety patrols, police officers and competent peers have all been relied upon to model correct pedestrian behaviors. Unfortunately, several programs invite exciting role models, such as police officers, into the classroom but never give children the opportunity to observe the role model in the real traffic environment.

Parents are probably the most influential role models and teachers. Because of this, this literature review has paid close attention to pedestrian programs in which parents are utilized. The teaching suggestions given to teachers have also been examined, as we assume that parents and teachers providing pedestrian instruction will need some of the same information. We have identified the following as critical teacher/parent needs for teaching preschool pedestrians: 
- knowledge of accidents involving preschool pedestrians;

- knowledge of preschool children's typical behaviors in traffic;

- understanding of preschool children's developmental needs vis-a-vis the environment (e.g., need for play but need for protection);

- framework for understanding how children learn pedestrian safety as well as knowledge of relevant teaching meithods and learning activities;

- knowledge of correct pedestrian behaviors and step-by-step sequences for executing those behaviors;

- knowledge of appropriate language to use when instructing children in pedestrian safety; and

- appropriate teaching materials.

This section concludes the overview of research in pedestrian programs for preschool children in the United States. The remainder of Chapter 4 contains detalled descriptions of individual pedestrian safety programs sponsored by public and private sectors in the United States.

\section{VI.H. Detailed Descriptions of Pedestrian Safety Programs}

The descriptions that follow summarize specific programs in (1) the public sector and (2) the private sector. They are ordered within sector group, alphabetically by state or program developing agency. The descriptions cover: authors, sponsors, date, age group, material reviewed, format, concepts, instructions, teaching methods, measurements of knowledge and behavior, and curriculum evaluation. 


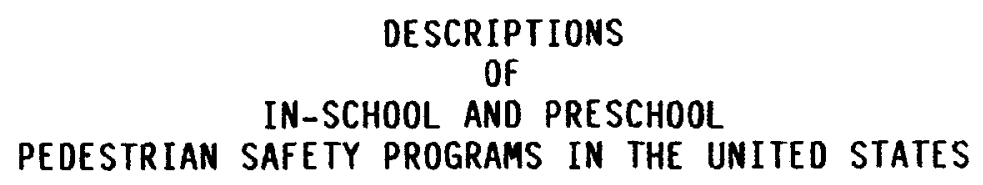


IN-SCHOOL PEDESTRIAN PROGRAMS 
Name of program: Pedestrian Safety Education for Alabama Youth

1. Primary authors: Driver and Traffic Safety Education/Division of Instruction, Alabama State Department of Education.

2. Sponsors: Office of Highway Safety, Alabama.

3. Date: January 1979.

4. Age of children in the program: $K-5$.

5. Kind of safety taught: Pedestrian.

6. Unit reviewed: $K$.

7. Format:

- The guide is divided into six grade levels for grades $K-5$. These are fourteen lessons in the kindergarten unit. Each lesson contains the following information: a behavioral objective, relationship of lesson to academic areas, list of materials, descriptions of suggested learning activities, words to songs and dittos. Two of the fourteen lessons provide activities to be used to assess children's knowledge.

- The dittos in the kindergarten section vary tremendously in terms of level of abstraction. Many dittos portray whole traffic scenes realistically. For example, one sees sidewalks with curbs, bushes and buildings, etc. Most of these scenes are drawn from the child's perspective rather than an adult's perspective or an aerial view. While many of these scenes depict urban and suburban environments, there are pictures of signs with no posts and no traffic environment context. There are two drawings of intersections which consist of little more than intersecting lines. Contextual clues are totally missing. The people in the dittos are either sketches of persons with no individual personalities or fully drawn pictures of children. No minority persons are shown. Clothing is not up-to-date, for women a lways are dressed in dresses and heels and girls always wear short $1 i t t l e$ dresses or skirts. It is interesting that the most obvious picture of a child breaking a traffic rule depicts a boy rather than a girl. A few scenes do not depict traffic situations at a11. They show Humpty Dumpty on a wa11, Jack and Jill on a hill, and the 01d Woman and her shoe, fruit, clocks, and seasonal clothes to put on "Chucky Clothes Conscious." 
8. Concepts taught in the following order (by lesson):

- Meanings of colors in the traffic environment (traffic light colors, colors for nighttime visibility and bad weather); stop, look, and listen before crossing the street

- Meaning and spatial position of traffic light colors

- Meaning of traffic light colors

- Safe and unsafe places to work and play

- Safe and unsafe places to work and play

- Safe and unsafe places to work and play

- Pedestrian rules

- Proper and improper pedestrian actions, meaning of signs

- Safety helpers and safety rules

- Safest route to school, personal information (address, etc.)

- Safety features (signs, crosswalks, etc.) in the traffic environment.

9. Pedestrian instructions given:

- Cross the street at the corner, not mid-block.

- Stop (not where to stop), look (not what to look for), and listen (not what to listen for).

- At the traffic light, stand and wait at a red light, wait at the yellow light, and look both ways (no mention of what to look for), then cross at the green light.

- Walk across streets.

- Look behind for turning cars when crossing the street.

N.B. The traffic methods taught vary slightly according to the teaching method used.

10. Teaching methods/materials used:

- Dittos (pictures of fruit, cars, Jack and Jill, traffic situations)

- Nursery rhymes (Humpty Dumpty) 
- Games such as "I spy"

- Songs

- Flannel board

- Models of traffic lights, and a traffic environment (model village)

- Films/filmstrip

- Discussion

- Stories

- Artwork such as fingerpaint, yarn pictures, drawing

- Riddle

- Role-play in simulated traffic environment in classroom or on playground

- Memorization of rules

- Talk by policeman who visits the class

- Map.

11. Measurement of chlldren's knowledge and behavior:

- Two lessons have activities which assess children's knowledge.

12. Evaluation of this traffic safety curriculum:

- This curriculum will be used in four large cities in Alabama to assess the curriculum's impact. 
ALASKA

Name of program: K-12 Traffic Safety Education Program

1. Primary authors: Alaska Department of Education-Division of Education Program Support.

2. Sponsors: Not listed.

3. Date: May 1979.

4. Age of children in the program: $K-12$.

5. Kinds of safety taught:

- Pedestrian

- Passenger

- School bus

- Bicycle

- Water and boating

- Basic first aid procedures

- Alcohol and traffic

- Drugs and traffic

- Snowmobile

- Motorcycle

- Driver and traffic safety.

6. Unit reviewed:

- Pedestrian safety.

7. Format:

- The entire curriculum is broken down by topic, not grade level. There are lists of general objectives for children in elementary, junior high, and driver age groups, however.

- The pedestrian unit contains sections on:

-- Reasons for pedestrian/vehicle accidents 
-- Simple statistics on numbers of accidents (e.g., "for five to fourteen year olds), accident reports indicate that 1) one half occur while crossing between intersections, 2) one third occur when crossing at intersections 3 ) others happen when pedestrians dart out between parked cars." )

-- Lists of safety practices to be developed

-- Lesson plans. Each lesson plan has a separate topic, followed by a list of suggested activities, ways to evaluate student knowledge, and list of materials.

-- Selections from Alaska State Laws Regarding Pedestrians.

- No dittos were included in the teacher's guide.

8. Concepts taught in the following order:

- Meaning of pedestrian

- Hitchhiking, its meaning and dangers

- Traffic signs and signals

- Rules and regulations such as walking on sidewalks

- Traffic hazards in bad weather, at night, around railroad tracks

- Dangers of strangers

- Community helpers such as policemen, reasons and meaning of traffic rules.

9. Traffic rules taught:

- Walk on sidewalks.

- When there are no sidewalks, walk on the left facing oncoming traffic and as far away from the roadside as possible.

- Cross streets at intersections.

- Stop on the curb, not in the street, to look for vehicles. Wait on the curb if a vehicle is coming. If all cars have passed or if all cars stop and the driver sees you, cross the street.

- Look left, right, and from the rear for cars before crossing the street.

- Follow signals if they are there. 
- Do not accept rides from a stranger or hitchhike.

- Keep away from parked cars.

- $\quad$ bey police and crossing guards.

- Play away from traffic, out of the street--in yards or playgrounds.

10. Teaching methods/materials used:

- Collage of pictures

- Discussion

- $\quad F j i m$

- Models of traffic signs and traffic environment

- Flash cards

- Booklet of signs

- Creation of poems and stories

- Memorization of rules

- Drawing

- Storybooks

- Pictures of streets, roads, traffic situations, etc.

- One demonstration of proper crossing behavior on a real street

- AAA's Safest Route To School program

- National Safety Council--"Make Sure They Can See You"

- Bulletin board, posters, skits

- Visit by policeman

- Visit to real street to observe policeman

- Crossword puzzle.

N.B. Activities are described very briefly, usually in one sentence. 
11. Measurement of children's knowledge and behavior:

- Suggestions for measuring children's knowledge is suggested for all of the six topics. Ways to assess children's knowledge include having children: give definitions; identify traffic signs; recite safety rules from memory; map out the safest route to school; recite reasons for safety rules. 


\section{CAL IFORNIA}

Name of program: California Guide For Pedestrian Safety Education.

1. Primary authors: California State Department of Education.

2. Sponsor: Office of Traffic Safety, California.

3. Date: 1978 .

4. Age of children in the program: $K-3$.

5. Kind of safety taught: Pedestrian.

6. Curriculum reviewed: Draft copy.

7. Format:

- The guide is divided into three sections-one for administrators, one for parents, and one for teachers.

- Volume I, aimed at administrators, suggests ways to make the traffic. environment around the school safer and whom to contact to bring about any needed changes. It also describes traffic control devices in the traffic environment, accidents that child pedestrians of ten have despite safety features in the environment, and precautions children should be taught to take to minimize danger. A glossary of traffic terms is provided as are also: a list of important facts about pedestrian accidents; a list of related careers in safety education; a list of federal, state, county, and city governmental agencies as well as private agencies which promote traffic safety; a true-false pedestrian safety education test.

- Volume II, which is directed at parents, informs them about their children's involvement in a traffic safety education program in school, gives brief summaries of the lessons their children will be taught in the school program and suggests activities that parents should do with their children in the real traffic environment near home in order to reinforce the lessons taught in school. The importance of parents as models is stressed. Parents are asked to report on their children's behavior after the program is completed by filling out the questionnaire provided and returning it to school. Another secion of this volume aimed at parents describes the kinds of traffic accidents befalling pedestrians of different ages and gives reasons for their accidents. A list of "Rules For Safe Walking" is also provided.

- Volume III is written for the classroom teacher. The teacher is told how frequently to teach the lessons during the year, how long each lesson should take, and for which grade level(s) each lesson is 
appropriate. Seventeen ungraded lessons follow. Each provides a description of the concept to be taught, desired teacher and student behavior during the lesson and appropriate reinforcement to be provided by the parent. The lessons are action-oriented; most take place in the traffic environment close to school. Very few dittos are provided. Those are of traffic lights (with no posts or traffic environment) and of "Walk/Don't Walk" signs.

8. Concepts taught in the following order:

- Pedestrian and vehicle surfaces (i.e., sidewalk, curb, bike path, street) and places of vehicle/pedestrian conflicts (1.e., alleys and driveways, etc.)

- To stop before crossing any place where there is a potential vehicle/pedestrian conflict

- Characteristics of different intersections

- Places where vehicles will park or travel

- Hazards of parked or standing vehicles and cues that tell pedestrians that vehicles might move

- The existence and proper use of marked crosswalks

- Not to cross the street until there is zero traffic moving

- How to recognize approaching vehicies and vehicles that have passed

- How to recognize turning vehicles and predict where they will turn

- How to recognize approaching vehicles and decide when to wait and when to cross

- How to use traffic signals at intersections--the traffic light and pedestrian "walk" and "don't walk" signals

- How to recognize and locate the direction of sounds

- How to recognize sounds of traffic

- How to help a friend search and detect approaching vehicles

- To obtain eye contact with drivers before crossing in front of any stopped vehicle 
- Not to dart into the street mid-block or dash into the street at an intersection--how to cross the street safely

- Safest route to school.

9. Pedestrian instructions. given:

- Walk on the sidewalk.

- Stop at the edge of the street and before other pedestrian/vehicle conflict areas before starting to cross.

- Recognize vehicles which are approaching, passing; turning, and leaving.

- When crossing the street, look and listen in all directions from which approaching vehicles might come.

- When no vehicles are moving on the street, it is the best time to cross.

- Avoid crossing the street in front of moving vehicles.

- Cross the street at the corner.

- Use a marked crosswalk when available.

- Don't step into the street to cause vehicles to stop.

- On a street with several lanes to cross, cross each lane separately and carefully.

- Walk across the street; don't run.

- At an intersection with a traffic signal, cross the street only on a new green light.

- Where there's a pedestrian signal, start to cross only when the signal says "Walk." Finish crossing or stop on an island when the signal changes to "Oon't Walk."

- When a traffic signal has a pedestrian push button, push the button to get permission to cross.

- When the view of the street is obstructed, walk to the corner of the obstruction to check for approaching traffic while standing in an area protected by the parked vehicle.

- Before crossing the street make certain that any approaching driver sees you and comes to a complete stop or passes by. 
- While en route to and from school, follow the safest route to school as agreed to by the parent.

10. Teaching methods/materials used:

- Walk in real traffic environment near school for each lesson

- Discussion about traffic environment while on walks

- Four safety games--creating and utilizing a simulated traffic environment in a playground

- Praise when children perform traffic safety skills successfully

- Tape recording of traffic sounds

- Magazine picture collage of things which make sounds

- Students instructing other students

- Films--"Street Talk" or "Watch Out" suggested for one lesson

- Map of route to school.

11. Measurement of children's knowledge and behavior:

- Observation of children's behavior during learning activities.

12. Evaluation of this traffic safety curriculum:

- Margaret Hubbard Jones and G.A. Fleischer from the Traffic Safety Center at the University of Southern California evaluated "The California Pedestrian Safety Curriculum." Their abstract is as follows: "The California Pedestrian Safety Curriculum was evaluated by means of an experimental design, using specially developed measuring instruments based upon the objectives of the curriculum and the critical pedestrian behaviors identified in in-depth accident investigations. Eight schools, stratified on socioeconomic level, were randomly assigned to training and nontraining groups. Students in grades kindergarten through three were tested for both knowledge and real-world behavior immediately before and immediately af ter the month-long training period. Three new, nonverbal knowledge tests were developed. A reliable performance measure for coding of critical behaviors effected a very significant, though not large, improvement in nonverbal knowledge of pedestrian safety practices. There was no demonstrable improvement in real traffic performance. Recommendations are made for curricular improvement before instituting a field test of the curriculum." 
COLORADO

Name of Program: Denver Pedestrian Safety Program--Wise Walker Program

1. Program developers: Applied Science Associates, Inc./Denver Police Department.

2. Program sponsors: Colorado Division of Highway Safety/NHTSA.

3. Materials developers/producers: Applied Science Associates, Inc.

4. Date: 1976-1980.

5. Age of children in the program: $K-3$.

6. Kind of safety taught: Pedestrian.

7. Materials reviewed:

- Denver Pedestrian Safety Program Teacher's Guide

- The Wise Walker Activity Book

- A Message To Parents...about Pedestrian Safety from the Denver Pedestrian Safety Program.

8. Components of the program:

- A massive public information campaign to alert the public to the problem and suggest ways to prevent accidents

- Traffic engineering modifications at various locations in the city of Denver

- Selective enforcement of pedestrian and driver rules at high-risk locations

- Educational programs aimed at high-risk groups, especially children ages five to nine and the elderly. In addition to the program provided for five to nine year-old children at school, the children's parents were sent a booklet which would enable them to reinforce the same concepts taught at school in the home.

9. Format of the Teacher's Guide:

- The "Introduction" to the guide explains that children's play of ten brings them into the street and indirectly causes traffic accidents. The "Introduction" also informs teachers that "Auto-Pedestrian collisions are the leading cause of accidental death among children in the United States" (page 1). Next, the reason for the Denver Pedestrian Safety project and its four program components (cited 
abovel are explained to the teacher. This is followed by an explanation of the nature of child-pedestrian accidents--the prevailing type of accident (dart-out midblock), when these accidents occur, where they occur, and under what conditions they occur.

- The "Overview of the School Program" discusses the two traffic skills to be taught in the program: (1) how to cross a street at an intersection; and (2) how to safely enter the street at midblock. The main program objective--the stop-search--detect procedure--is explained, followed by a detailed step-by-step explanation of how to execute a safe mid-block street entry, and finally, a step-by-step explanation of how to safely cross at intersections. For fur ther clarification a diagram is provided to show directions a car might turn at an intersection, thus showing the appropriate places for pedestrians to look for vehicles before and as they (the pedestrians) cross the street.

- The "What the Program Contains" section gives an overview of the teaching methods and learning materials to be used in the program. of all materials and experiences provided, the learning experiences considered the most important are those experiences which teach children safe mid-block street entry and intersection crossing skills, i.e., correct behaviors. Practicing these behaviors in a real traffic situation, a real street made safe by barricade blocking traffic flow, is stated to be the optimal place to teach these skills. However, teachers are told that due to the costs of providing police to barricade streets, this setting cannot be used; mid-block street crossing skills are, therefore, taught in a simulated environment (playground or gym) which is made to look as much like the real environment as possible. Street crossing procedures are taught, however, at real intersections.

- The "Schedule of Activities" explains how frequently training sessions should be given over the course of a year and how long sessions should be. Teachers are also given the names of persons and departments who can assist them if they encounter difficulties while teaching the program. 
- The next section gives a brief summary of the theoretical basis of the program. The main theories espoused are:

-- Sometime between the ages of five and nine children move from the pre-operational. to the concrete operations stage of intellectual development. Children at both stages have limited abilities, thereby affecting the traffic safety concepts they can be taught. For instance, the guide states that pre-operational children are unable to make speed-distance judgments, but advocates giving them experiences which will later enable them to make such judgments.

-- The five to nine age group is known for its literalism and ritualism. Children at this age can follow rules--and will do so. if they are not too restrictive.

- Children this age need many opportunities to practice following rules correctly. They need verbal feedback as well.

- "Preparing For Teaching the Program" lists materials to be supplied by the project and school and explains how to set up the simulated street environment on the playground or in the gym.

- The next section explains the purpose of playing games during the safety education program--not because games are entertaining but because children's accidents happen so of ten when they are at play. Games are used to simulate the conditions under which children's accidents occur. The games also make it possible to integrate the traffic safety program into the ongoing physical education program. The games chosen are selected because they cause children to enter the simulated street on a random and relatively frequent basis. Children's safe street entry skills are scored every time they leave the game and enter the simulated street.

- "Arranging For Extra Personnel" suggests utilizing the services of teacher assistants and parent volunteers both during the safety games teaching mid-block street entry and during instruction at intersections.

- "Informing the Child's Parents" reminds the teacher to distribute pamphlets to the children's parents.

- "Program Evaluation" explains the importance of keeping records of the names of children in the program, their birth dates and number of sessions in the program they attended. 
- "Final Cautions" states that children learn by watching and by doing. The importance of teacher and peer models and children's needs to practice behaviors repeatedly are stressed. Emphasis is also placed on making sure that each child performs the learned behavior correctly.

- The last half of the guide contains lesson plans for 12 sessions and an appendix with instructions for several games such as "Catch on the Fly" and "Corner Kick Ball." Eight of the twelve sessions are devoted to mid-block street entries while four pertain to intersection crossings.

10. Concepts taught in the Teacher's Guide in the following order:

- Correct way to conduct the left-right-left search for cars from the curb--why to do it and how to do it.

- Why it's important to enter the street safely

- Parked cars interfere with a pedestrian's view of moving traffic when the pedestrian is standing on the curb

- Correct way to conduct the left-right-left search sequence when parked cars are present at the curb--walk to the outer edge of the parked car and search from there

- Importance of tracking vehicles until they've gone past

- Dangers of parked cars--they might move and accidentally hit a pedestrian behind or in front of them

- Ways to detect whether a parked car may move (signal, engine sound, car moving)

- Safe and unsafe times to enter the street mid-block (what constitutes a safe or unsafe time is not described for the teacher)

- To enter the street mid-block from both the curb and from the edge of parked cars after performing the left-right-left search

- Correct sequence of behaviors to detect moving vehicles from all directions at an intersection with no traffic signal (left-rightleft --front-back--left-right-left) and rationale for sequence

- The different directions vehicles may move at intersections

- Meaning and use of crosswalks

- Different kinds of intersections--with and without crosswalks 
- That it's incorrect for pedestrians to triy to stop traffic

- Whether accompanied or not, to search for cars oneself

- Halking rather than running across intersections

- Meanings of red, yellow, and green signals of the traffic signal light and appropriate pedestrian behavior

- Meanings and pedestrian use of "Walk" and "Don't Walk" lights

- Correct use of pushbutton on traffic signal light

- Correct sequence of behaviors to detect moving vehicles at an intersection with a traffic light signal present (left-rightleft--front-back--left-right-left).

11. Pedestrian instructions given in the Teacher's Guide:

- When you have to enter the street mid-block, first stop at the curb, search left-right-left for vehicles and if it's safe, enter the street. (This rule applies when no cars are parked along the curb.) If you detect vehicles coming, track the vehicles until they've gone past, do another left-right-left search and enter when it's safe.

- When cars are parked along the curb and you have to enter the street mid-block, walk to the outer edge of the cars, search left-right-left for vehicles, and enter the street if it's safe. If vehicles are coming, track vehicles until they've gone past and do another left-right-left search until it's safe to enter.

- Hatch parked cars for signs they may move and stay away from those that look as if they may move.

- Don't enter a street or cross a street when vehicles are coming.

- Cross a street at the corner--at an intersection.

- Use the crosswalk to cross the street if one is provided.

- At an intersection with no traffic signal light present, stop at the corner, search left-right-left--front-back--left-right-left for vehicles. If vehicles are coming, track them until they've passed, and do another search. When no vehicles are coming, walk, don't run, across the street.

- At an intersection with a traffic signal light present, stop when the red and yellow signals face you. If the green signal faces you, do the intersection search sequence and then if it's safe, cross the street. 
- Continue walking across the street when the "Walk" sign changes to "Don't Walk," but don't start to cross the street if you're about to cross and the "Don't Walk" sign comes on.

- Don't try to stop traffic.

- If you're walking with another person, you need to stop and do the search for cars sequence yourself rather than relying on that other person.

- If the traffic signal light has a button, push it to activate the "Wa lk" light.

12. Teaching methods/materials used:

- Practicing correct behaviors in simulated traffic environment using games to simulate play conditions (most of the time)

- Practice crossing at a real intersection

- Consistent, honest feedback to each child

- Insistence that the child practice the behavior correctly

- Having the child verbally repeat aloud the search sequence as he/she searches for cars

- Teacher modeling correct behavior

- Peer group modeling correct behavior

- Close physical and verbal guidance given by the teacher to the child as he/she performs the street search sequence

- Questioning children to make sure children know they are searching for cars etc.

- Discussion/explanation

- Trips to real street to observe traffic, safety features, etc.

- Giving children as much responsibility for deciding when to cross the street as is safe and possible

- Reminding children to think and act independently when crossing the street

- Lots of repetition--practicing the same behavior or skill many times both separately and in the context of different games. 
13. Format of The Wise Walker Activity Book:

- This 11" $\times 14^{\prime \prime}$ 16-page booklet has a variety of activities for children

-- dot-to-dot activities showing correct or incorrect pedestrian behavior

-- comic stories in which Wise Walker instructs children in crossing the street and intersections

-- large illustrations each of which covers an entire page

-- a maze

- picture with cars hidden in illustration

-- contrasting pictures showing correct and incorrect pedestrian behavior

-- comic story in which an older brother counsels the younger that safety. is more important than toys

-- a back page which gives all the answers to the games.

- Illustrations show children of different races and ethnic backgrounds and up-to-date clothing. Illustrations of traffic environments have a medium amount of detail so that contextual cues aid the reader in understanding which lines represent streets, which represent sidewalks, etc. Some illustrations are more realistic than others. Some cars are given eyes, for example.

14. Concepts taught in the Wise Walker Activity Book:

- Dangers of walking between parked cars

- Danger of running into the street from between parked cars

- Stopping at the curb and looking left-right-left before entering the street

- Crossing at intersections

- Children are smaller than cars and therefore can't be seen when they stand behind cars

- When a bully is chasing you, don't run into the street--run on the sidewalk if you have to run

- Dangers of riding big wheels into the street

- Stopping and looking in all four directions before crossing an inter section

- Walking rather than running across the street 
- When cars are parked next to the sidewalk, walking out to the edge of the cars to search for cars rather than attempting to search for cars from the curb

- Looking for cars yourself rather than relying on an adult to do it

- Helping younger children be safe by keeping them from running into the street

- That the safety of people is more important than the safety of toys; toys are replaceable but people are not

- Cars, being bigger than children, can hurt children very badly in a collision.

15. Teaching methods used in the Wise Walker Activity Book:

- Games such as dot-to-dot, mazes

- Comic stories

- Illustrations

- Children drawing their own cartoons.

16. Format of A Message To Parents... About Pedestrian Safety (parents' book (et):

- The letter to parents from the Denver Chief of Police briefly states child pedestrian accident facts and introduces the booklet as a guide which aims to inform parents about the causes of child pedestrian accidents and help parents take steps to prevent those accidents.

- The "Introduction" states that the traditional traffic safety maxim taught to children "Don't go into the street" is incompatible with school-age children's developmental needs and every day patterns and does not prevent this age group from having pedestrian-auto collisions. It introduces the new approach to traffic safety education taken by the Denver Public Schools, which teaches children how to enter or cross the street.

- "The Nature of Child Pedestrian Accidents" describes the conditions under which "dart-out" accidents occur.

- "Basic Safety Rules" outlines the stop, search, and detect procedure taught in the Denver Child Pedestrian Program, and the following sections provide additional information on each step of the procedure. 
- "Note Concerning Preschoolers" reiterates that the street entry/crossing procedures are intended to be taught to school-age children, not preschool children. The guide recommends the following for children under five years of age:

- "If they must cross a street, they should always be accompanied by an adult or a responsible child who is at least 14 years old.

- They should not be permitted to play outdoors without the direct supervision of an adult or responsible children 14 or older." (p. 13)

- "How You Can Help" suggests the following ways parents can reinforce the safety habits their children are learning at school:

-- Set a good example.

-- Comment when your children use correct traffic safety procedures.

-- When crossing the street with children, encourage them to decide when to cross and tell them whether their choices were wise or unwise and why.

-- Play with your children outside to insure that they use the correct safety procedures before entering the street.

-- Set guidelines relating to your children's play near the street.

- Put the Wise Walker Card (which states the causes of child pedestrian-auto accidents and ways that parents can prevent such accidents from occurring) in a prominent place to remind other people in Denver of the importance of child pedestrian safety.

- The Wise Walker Card described above is printed in both English and Spanish. It is inserted in the booklet.

- The booklet contains many of the illustrations from the Wise Walker Activity Book, plus diagrams to enable parents to visualize the visibility problems which beset both pedestrian and driver at mid-block locations where cars are parked at the curb.

17. Measurement of children's knowledge and behavior:

- Children's street crossing behaviors were evaluated before and after exposure to the educational program in order to evaluate the effectiveness of the school program. The method chosen to assess children's behavior was an observational one; each child was observed while taking an empty pop can to a recyciing truck parked across the street from the school. Data showed that children in all five schools made a significant increase in stop and search behavior, 
although the results from one school were extremely deviant from the other four. Children there showed a 74-fold increase in stop-and-search behavior at the curb as opposed to a geometric average of 3.05 for the other schools. (For further information see "Test of the Effectiveness of the Denver Pedestrian Safety Project Educational Program for Kindergarten Through Third Grade children in Denver Public Schools," by Applied Science Associates, Inc.)

18. Evaluation of this traffic safety curriculum:

- Accident data revealing whether children who have not participated in the program are involved in more accidents proportionately than children who have participated in the program will determine the effectiveness of the program. As described above, pre- and post-observational data of children's street crossing behavior at the curb were analyzed, showing favorable results. In addition, teachers in the program were interviewed to find out their level of satisfaction with the program. Most teachers were reported to like the program. The few dissatisfactions with the program concerned some equipment used in the games, the time children spent waiting in line for their turns to toss the ball or cross the street, and the long amount of time it took children, especially younger children, to conduct the intersection search procedure. 


\section{CONNECTICUT}

Name of program: A Preschool Traffic Safety Club

1. Primary: authors: Kathleen De Meo and Gregory Futoma from the Office of Safety Education in the Department of Public Safety, Hartford, Connecticut.

2. Sponsor:- Connecticut Governor's Highway Safety Representative/NHTSA.

3. Date: 1980-1981.

4. Age of children in the program: Preschool.

5. Kinds of safety taught:

- Pedestrian

- Passenger.

- Bus safety.

6. Materials reviewed:

- None, as the newsletter had not been developed at the time of inquiry.

7. Concepts to be taught:

- Safe play areas

- Crossing streets

- Safest route to school

- And others.

8. Teaching methods/materials used:

- During each month of 1980 for seven months, four-year-old children were sent a newsletter containing games, puzzles, and factual information. Parents were expected to read the news letter to the children and help them with games and puzzles. One section of the newsletter is intended for parents only; this section consists of traffic safety instructional activities for parents to use with their children in actual traffic situations. 


\section{CONNECTICUT}

Name of program: Preschool Education for Safety

1. Author: Sarah Greer, teacher at the Gan School in New Haven.

2. Sponsor: Highway Safety Division of Connecticut.

3. Date: Started December 1979.

4. Age of children in program: 2 years 9 months to 5 years.

5. Kind of safety taught:

- Pedestrian

- Tricycle

- Passenger safety for urban children.

6. Material reviewed:

- None, as materials were in the process of being developed at the time of inquiry.

7. Concepts which may be taught:

- Crossing streets at intersections

- Traffic lights

- Seat belts

- Boarding and disembarking from vehicles

- Where to ride tricycles.

8. Teaching methods/materials which may be used:

- Workbook with puzzles, coloring, and pasting activities for children

- Teacher's guide that provides traffic safety concepts to be taught, songs, learning activities to be used on safety walks, games, fine motor activities

- Slide production.

9. Measurement of children's knowledge and behavior:

- Once children have been taught all the concepts, their behavior in traffic will be informally observed by teachers. 
10. Evaluation of this traffic safety curriculum:

- No formal kind of pre- and post-test evaluation will be given. As cited above, they will observe children's behavior after training has been given. 


\section{CONNECTICUT}

Name of program: Safety program in Vernon, Connecticut, a local program under the leadership of officer William Yetz.

1. Sponsor: Highway Safety Work Program from the State of Connecticut Department of Transportation gave the original grant of $\$ 2,926$ in 1970. In 1973 the town of Vernon assumed all costs.

2. Date: Since 1970.

3. Age of children in the program: $K-6$ in school program, four to sixyear-olds in the Safety Town summer program.

4. When program is taught: Mornings and afternoons for six weeks in the summer and through occasional visits to school (N.B. Each Safety Town summer course lasts one week).

5. Kinds of safety taught:

- Pedestrian

- School bus

- Bicycle.

6. Materials reviewed:

- Color Me Safe coloring book and Betsy and Bill and the Nice Bad Man, a book teaching children about the dangers of strangers.

7. Format of the Color Me Safe coloring pamphlet:

- Color Me Safe focuses on teaching safety features in the traffic environment--traffic lights, signs, crosswaiks, and "Walk/Oon't Walk" lights. In only one instance is the safety feature shown in context. in the traffic environment. Generally speaking, each page has a picture of one safety feature such as a traffic light with no post. Some pages have dot-to-dot activities, which when connected form signs. The traffic lights are humanized; they have smiley, angry/sad, or pained facial expressions depending on whether the red, green, or yellow light is on. The only traffic environment shown depicts a car coming to a crosswalk that has a smiling face in it. 0 ther than the sidewalk and dotted line on the road, no other contextual clues are given.

8. Concepts taught:

- Meanings of colors in traffic lights

- Meanings of signs 
- Meaning and use of crosswalk

- Where to cross the street (at corners)

- Dangers of strangers

- Concepts taught in Safety Town Program (walking on sidewalk, where to cross the street, etc.).

9. Teaching methods/materials used:

- Jiminy Cricket film by Walt Disney

- Coloring book

- Officer Yetz visits school classrooms and talks with children

- Safety Town, simulated traffic environment scaled down to child size

- Betsy and Bill and the Nice Bad Man, a book written by the Vernon police department.

10. Evaluation of this program:

- No formal evaluation. However, according to officer Yetz, in the last four or five years, Vernon has had no fatal pedestrian or bicycle accidents. 


\section{DELAWARE}

Name of program: Safety Action

1. Authors/sponsors: Delaware State Department of Public Instruction in cooperation with the U.S. Department of Transportation Federal Highway Administration National Highway Safety Bureau.

2. Date: May 1968.

3. Age of children in the program: $K-6$.

4. Kinds of safety taught:

- Pedestrian

- School bus

- $\quad$ icycle

- Passenger.

5. Unit reviewed:

- Walking to and from school and school bus safety (which contain pedestrian safety information)

6. Format of entire book let:

- The introduction outlines basic goals of the program, best ways to teach traffic safety, and ways to use the guide.

- The next section lists elementary school children's characteristics as related to safety.

- "Responsibilities of the Safety Team" describes roles and responsibilities of the school personnel, parents, school safety council, bus driver, etc.

- Each section on traffic safety tells the teacher when to teach the unit, provides accident statistics to emphasize the need for that type of traffic education, lists safety habits to be developed in the children, and suggests several learning activities related to the lesson.

- At the end of the booklet there are lists of sources of traffic safety materials such as films, teacher guides, and brochures and sections from the Motor Vehicle Laws of the State of Delaware related to pedestrian, bicycle, and school bus safety. 
No dittos are provided within the teacher's guide.

7. Concepts taught:

- Obeying traffic safety features such as traffic lights and traffic authorities such as parents and policemen

- Identifying and acting upon traffic sounds

- Traffic safety features such as traffic lights, crossing guards, and dangers such as obstacles on sidewalks or roads

- Walking on the left side of the road facing traffic (no mention of why--lack of sidewalks)

- Where to walk (which side) on sidewalks

- Where to cross the street

- How to cross the street at guarded and unguarded intersections

- Safe routes/dangerous places to walk

- Dangers of strangers

- Dangers of strange animals.

8. Pedestrian instructions given:

- Obey police, crossing guards, etc.

- Look for safety features (such as traffic lights) and dangers (slippery roads, crowded sidewalks, etci).

- Walk along the left side of the road facing traffic (no mention why--in what situations).

- Walk on the right side of the sidewalk when other people on the sidewa ik approach you.

- Don't cross the street near parked cars:

- Don't dart out between cars.

- Don't cross the street mid-block.

- Don't walk in the street when sidewalks are available.

- Don't cross against trafic lights. 
- Don't cross when you're unsure how "new" the green light is.

- Don't accept rides or anything else from strangers.

- Don't play on the way home from school.

- Don't go in isolated areas such as alleys, fields, and deserted buildings.

- Stay clear of strange or unfamiliar animals.

- Look left-right-left before crossing the street.

- Halk across streets.

9. Teaching methods/materials used:

- Dramatizations of safety practices in classroom and assemblies

- Maps of community

- Visit to classroom by crossing guard or police officer

- Stories about preventable accidents

- Creative story-telling by students

- Orawing pictures

- Transparencies

- Writing safety jingles

- A walk around school to notice different types of traffic signs, lights, etc.

- Discussion.

10. Measurement of children's knowledge and behavior.

- No assessment instruments were suggested. 
Name of program: D.C. Walker

1. Sponsors: District of Columbia Department of Transportation and Metropolitan Police Department.

2. Date: Since 1978.

3. Age of children in the program: $K-3$.

4. Kind of safety taught: Pedestrian.

5. Material reviewed:

- $\quad$.C. Walker's Safety Fun Book

- Walk Safely with O.C. Walker (brochure)

- O.C. Walker slide presentation.

6. Format:

- $\quad$.C. Walker's Safety Fun Book is a seven-page book with activities such as a picture and word match-up, a safety picture story, story with a secret safety message buried in the letters, a "What's Wrong With This Picture!" activity, a traffic sign maze, a dot-to-dot activity, and a paper doll puppet cut-out.

- The Walk Safely with D.C. Walker brochure is a short pamphlet developed for children's parents. It contains lllustrations and information relating to seven kinds of accidents-intersection dash, multiple threat, vendor-ice cream truck, commercial bus related, dart-out, backing up, driver violation. Pedestrian responsibilities are emphasized. Nine safety rules are listed on the last page of the brochure.

- The D.C. Walker slide show reviewed was produced in 1978 by $\mathrm{Mr}$. George Douglas from the Lawrence Kaplan Advertising Agency. The hero of the show is D.C. Walker, an inner-city child who possesses magical tennis shoes which have made him the safest walker in the city. He's awarded a safety medal and is loved and imitated by thousands. D.C.'s mother narrates the (f lashback) story of how D.C. Walker's sneakers stopped him from doing inappropriate behaviors in traffic and made him observe safe pedestrian behaviors. This slide show is currently being revised to deemphasize D.C. Walker's magic tennis shoes. Then it will be used in grades K-6. Although D.C. Walker is a boy in the present show, current program managers emphasized the androgenous character of D.C. Walker and pointed out that D.C. Walker is light-skinned enough to be from many ethnic and racial groups. 
- The 0.C. Walker Program in elementary schools is carried out by local officer Friendly police officers. These officers are given training before they go into classrooms. The D.C. Walker Guide given to the officers describes the audio-visual equipment needed for the slide show, recommends an order for the program presentation, and suggests a discussion activity that the officer can do with the children and the D.C. Walker puppet. The discussion is to consist of true-false questions about appropriate and inappropriate pedestrian behaviors which the officer asks both the children and the puppet. The puppet and the officer are encouraged to explain why certain behaviors are safe and unsafe. After this activity, the officers are supposed to hand out the D.C. Walker Safety Fund Book (s).

- Sometimes the 0.C. Walker Program is taken to day care centers. The film is shown and children are encouraged to discuss and pantomime safe pedestrian behaviors. They also meet the $D$.C. Walker puppet.

7. Concepts taught in the D.C. Walker slide show:

- Where pedestrians should walk

- Where to cross the street

- Where to ride a Big-Wheels

- What to do when the ball rolls into the street

- How to scan for vehicles

- How to scan for cars when disembarking from a bus

- What to wear at night.

8. Concepts taught/reinforced by the officer Friendly:

- Where pedestrians should walk

- Where to walk when no sidewalks exist

- Where to play

- How to cross the street at a traffic light

- The importance of walking across the street

- Dangers of walking at night

- How to behave when an ice cream truck comes

- Where big wheels and tricycles should be ridden 
- What a pedestrian is

- Who the safest child in Washington, D.C., is.

9. Pedestrian instructions given:

- Walk on sidewalks, not in the roadway.

- If no sidewalks exist, walk on the side of the roadway facing the cars.

- Never play in the street.

- To cross a street at a traffic light, wajt until all cars have stopped for the red light, and the light facing you is green. Cross on the green light only after you've looked both ways to see that no cars are turning the corner.

- To cross a street which has no traffic signals, stop at the edge of the street, look left-right-left, and if no cars are coming you can walk.

- Cross streets only at the corner.

- Ride Big Wheels and tricycles on the sidewalk, not on the street.

- Walk, don't run, across the street.

- When you're out walking at night, wear white clothing, carry a flashlight and wear reflective material.

- When getting off a bus, stop at the edge of the bus and look left-right-left for approaching cars. When it's safe (not defined), get off the bus and walk to the sidewalk.

- When a ball rolls into a street, don't run after it.

- Don't run across the street at mid-block to get to the ice cream truck.

- Don't run out from between parked cars.

10. Teaching methods/materials used:

- Slides

- Discussion

- Role-play looking left-right-left in the classroom

- Story

- Interpreting adult pictures of traffic situations 
- Sign maze

- Dot-to-dot activity

- Parent participation.

11. Measurement of children's knowledge and behavior:

- No assessment instruments were suggested.

12. Evaluation of this traffic safety curriculum:

- Officer Friendly gives teachers an evaluation form to fill out.

- The District of Columbia Department of Transportation is looking at accident rates of children in the city. It is not clear whether a

- change in accident statistics will be used to evaluate the program's effectiveness. 


\section{FLORIDA}

Name of program: K-3 Traffic Safety Education Resource Curriculum (Florida)

1. Primary Authors: Transportation and Safety Unit, College of Education at Florida State University for the Florida Department of Education.

2. Sponsors: Florida Governor's Highway Safety Commission and U.S. Department of Transportation.

3. Date: 1974 .

4. Age of children in the program: $K-3$.

5. Kind of safety taught:

- Pedestrian

- Bus safety

- Car passenger

- Bicycle.

6. Unit reviewed:

- Pedestrian.

7. Format:

- None of the four lessons in the pedestrian section is broken down by age or developmental level; each teacher is expected to do this $\mathrm{him} /$ herseif. Each lesson identifies lifelong traffic behaviors taught in the lesson, behavioral objectives, vocabulary pertinent to the lesson, relevant rules and regulations, learning experiences and list of materials to be used. Each lesson actually contains many concepts to be taught; they are taught by giving children hypothetical situations and discussing with them what should be done.

- With the curriculum guide come large glossy posters and black and white dittos. All depict the traffic environment and traffic situations in a highly realistic fashion.

- The posters show streets and roadways which are black with white dotted lines, white crosswalk lines, and white sidewalks, for example. Other contextual clues such as shrubs, stone walls, buildings, and fences are given which make the traffic scenes look more realistic. Rural, suburban, and urban traffic environments are shown, along with one overhead drawing of a city and pictures of a cut-away bus and cut-away car. The posters are colorful, sharp, and glossy. The few pedestrians shown are wearing up-to-date clothes and are modeling appropriate pedestrian actions. 
- The dittos are also up-to-date in their presentation of typical current traffic environments such as the shopping center, their portrayal of minority persons, and their presentation of non-sexist and up-to-date wearing apparel. A wide variety of traffic environments are shown--inner city, rural and suburban. The tropical as well as non-so-tropical florida landscape is shown. Pedestrians cope with traffic in different weather conditions. Both proper and improper pedestrian behaviors are presented. In summary, these dittos are extremely versatile, up-to-date, and comprehensive.

8. Pedestrian instructions/concepts taught in the following order:

- Stop before crossing the street.

- Stay on the curb until it is safe to cross.

- Look and listen for approaching or turning vehicles before and while crossing.

- Yield right-of-way to vehicles.

- Base a decision to cross the street or wait on the curb on the speed and distance of the vehicles present.

- Look for vehicles approaching from all directions even while crossing in a crosswalk.

- Walk across the street.

- Cross streets at the corner, at crosswalks on corners, or mid-block where there is a crosswalk.

- Yield right-of-way to all vehicles when crossing in rural areas where there are no crosswalks.

- At corners with traffic lights, cross on the green signal after all vehicles have stopped.

- At corners with "Walk"-"Don't Halk" signals, cross when vehicles have stopped.

- Obey traffic officers, crossing guards, etc.

- Walk inside crosswalks.

- Cross one street at a time at intersections instead of crossing diagonally.

- Walk on sidewalks whenever available.

- Keep to the right when walking on the sidewalk. 
- Walk single file on the side of the road facing traffic when there are no sidewalks.

- Look and listen for warnings and hazards such as a car backing out of a driveway, a turning vehicle.

$\therefore \quad$ Wear light $c$ lothes and reflective tape on clothing at night.

- Carry a flashlight if walking at night.

- Carefully select where to stand at night, 1.e., so you can be seen by cars.

- Cross the street at a lighted area at night.

- Take extra precautions in bad weather.

- Play as far away from streets and sidewalks as possible.

- Choose the safest route to school.

- Whenever possible, walk in areas with light traffic.

- Allow enough time to get where you are going.

- Carry packages and wear clothes so they don't block your vision.

- Be alert for signs of emergency vehicles.

- Cross railroad tracks carefully.

- Pet only animals you know are safe.

- Know your name and address.

9. Teaching methods/materials used:

- Discussion

- Instructional aids--large fold-out traffic scenes with cut outs of people, vehicles and stgns

- Hazard awareness cards--pictures of hazardous traffic situations.

10. Evaluation of children's knowledge and behavior:

- Evaluation of children's knowledge is suggested by giving children more hypothetical situations (shown by instructional aids) to solve. 
FLORIDA

Name of program: Safe Street Crossing Program (Willy Whistle) in Miami Dade, F lorida

1. Curriculum materials:

- Safe Street Crossing Teacher's Guide

- Safe Street Crossing Activity Booklets (one for Kindergarten, one for older childrenl.

2. Developers: NHTSA and the Dade County Department of Traffic and Transportation.

3. Date: Since 1977.

4. Age of children in the program: $K-3$.

5. History of curriculum materials: The Safe Street Crossing Program builds upon the work of Applied Science Associates and Dun lap Associates on anti-Dart-out training.

6. Kind of safety taught:

- Pedestrian.

7. Materials reviewed:

- Teacher and kindergarten activity booket cited above.

8. Format the teacher's guide:

- The "Preface" presents facts about children's pedestrian accidents and explains why certain countermeasures were chosen for this program.

- The first section gives an overview of the behavioral objectives of the program, the training plan for K-third grade, the curriculum, the order of training, and the need for parent participation.

- The second section, consisting of seven basic lessons, describes learning activities and materials.

- A progress chart, an activity log and a program evaluation form are included at the end of the booklet. Willy Whistle stick-on tokens are provided separately.

N.8. In addition to the teacher manual teachers are given training in the curriculum prior to the time they teach children. 
9. Format of the children's 14-page workbook.

- Each work book page has few pictures and few lines of writing.

- There is space for children to draw a picture.

- Spaces are provided where children should paste tokens symbolizing mastery of a lesson.

- Pictures of traffic environment in most cases are quite realistic. Many contextual clues such as trees, grass, fences and buildings are given so that most sidewalks can easily be differentiated from streets and from the grass. While signs without posts or traffic environments are shown in a matching game, there are pictures of signs firmly rooted in the ground in a traffic scene.

- A few concepts such as walking facing traffic if there are no sidewalks and traffic safety features such as stop and yield signs that are introduced in the children's work book are not integrated into lessons in the teacher's guide.

- Many of the activities in the booklet are perceptual activities. Children are asked to find two signs that are the same and two willy Whistles that are facing the same direction. Perceptual motor tasks are given such as drawing a line between two lines that go in different directions.

10. Concepts taught/pedestrian instructions given:

- Stop at the curb, (taught in both teacher's and and children's book lets).

- Look left-right-left for approaching traffic (emphasized in teacher's lessons).

- Walk across the street if the street is clear of cars (taught in both children's and teacher's booklets).

- Notice sounds and appearances of parked cars that have engines going and may move (mentioned only in teacher's lessons).

- Walk on the sidewalk (mentioned in both children's and teacher's book let).

- If there are no sidewalks, walk facing traffic (taught in children's book let on ly.).

- Traffic symbols--stop sign, pedestrian crossing, don't walk, yield (taught in children's booklet only).

- Cross at corners (taught only in children's booklet).

- Cross mid-block (taught only in teacher's lessons). 
11. Rationale for concepts taught:

- Most of the five to nine-year-old children killed in traffic accidents in Dade County are killed in dart-out accidents in residential neighborhoods. Since children are crossing and are going to continue to cross mid-block and since they lack the skills to do this safely, the countermeasures for the Willy Whistle program are directed at teaching children how to cross mid-block and to deal appropriately with the following traffic situations with which children have difficulty (1) chasing play equipment such as balls into the street; (2) being called from across the street; and (3) being chased into the street.

12. Teaching methods/materials used:

- Lecture in assembly

- Willy Whistle Movie

- Willy Whistle Song

- Discussion

- Traffic games simulating three traffic situations listed above played on a private drive or barricaded street or on a simulated street with model cars indoors

- Work book activities which work on perceptual abilities such as matching identical pictures of traffic symbols and interpreting pictures of traffic situations, on knowledge of safety rules and on small muscle coordination

- Teacher modeling correct behavior

- Individual participation in that each child takes a turn crossing the road (as opposed to leading the group of children across the road)

- Tokens of Willy Whistle, used as reinforcers

- Children advising and evaluating others doing the street crossing task in a simulated environment

- Final lessons on real street to review concepts learned

- Ouring this first year in the program, children are given initial training of seven 40-minute lessons; make-up for lessons missed; follow-up training. During the following years of training students take the refresher course.

- In general, lessons progress from abstract discussions indoors to activities using a simulated traffic environment indoors to activity lessons in the real traffic environment (outdoors). 
13. Parent participation:

- Parents are asked to reinforce children with tokens when children perform well in traffic. There is not much emphas is on parents teaching their children traffic safety; more emphasis is on teaching parents their driving and pedestrian responsibilities.

14. Measurement of children's knowledge and behavior:

- Teacher observation of children's behavior during lesson exercises.

15. Evaluation of this traffic safety program:

- The Miami-Dade Project is doing a four-level evaluation, looking at: (1) number of children in and completing the program; (2) the results of interviews with children in the program; (3) observations of children's actual street crossing behavior as they walk home from school; and (4) accident reports which project staff retrotype themselves. The evaluation will take place between 1979 and 1981 . Part of the study involves an analysis of the knowledge and behavior of children who have been exposed to the entire program, children who have had partial exposure to the program and children who have had no exposure to the program (the control group). 
Name of program: Although there is no pedestrian program for the preschool or elementary age groups, there is a coloring book Pedestrian Safety Coloring Book

1. Author/sponsor: Georgia State Patrol.

2. Date: O.K.

3. Age of children for whom the book is intended:

- Preschool

- Kindergarten.

4. Kind of safety taught: Pedestrian.

5. Material reviewed: Pedestrian Coloring Book.

6. Format:

- This-nine page booklet has generally one picture with a few words beneath on each page.

- Pictures show a policemen, trooper, patrol car, traffic light, eyes, signs, and a stranger offering a bag of candy. The "Friendly Policeman" and "Friendly Trooper" look almost identical. Both are whistling (you can see two musical notes indicating their whisting, a concept which only an adult would understand). Slight differences are apparent from close scrutiny (their belts, badges, hats, pants, etc., are slightly different). They are not placed in any environmental or situational context to alert children to their law enforcement roles. The traffic light and traffic signs also have no posts or traffic environment context. In no place is a pedestrian situation shown, displaying either appropriate or inappropriate pedestrian behavior. No parts of the traffic environment except a few signs are taught through illustrations, and as mentioned earlier, the signs are not shown in a traffic environment.

7. Concepts taught:

- What a policeman looks like, that he's friendly

- What a trooper looks like, that he's friendly

- What a patrol car looks like

- What a traffic light looks like 
- That eyes look (nothing about what to look for)

- What stop, school, and railroad signs look like

- Don't accept rides or lifts from strangers

- The child is asked to pledge to look (not what to look for).

8. Pedestrian instructions given:

- Look (scan) when you come near a street (not what to look for).

9. Teaching methods materials used:

- Coloring

- Parents may be expected to read the words to their children and explain the intended concepts.

10. Measurement of children's knowledge and behavior:

- No assessment instruments are suggested. 
Name of program: Idaho Traffic Safety Education Curriculum Guide

1. Authors: Idaho State Department of Education and various educators in Idaho.

2. Sponsors: Idaho Traffic Safety Commission/NHTSA.

3. Date: Written and revised 1976-1978.

4. Age of children in the program: $K-6$.

5. Kind of safety taught:

- Bicycle

- Passenger (bus and car)

- Pedestrian.

6. Unit reviewed: K-Pedestrian.

7. Format:

- Each lesson in the "Pedestrian Unit" provides the teacher with: a single concept to be taught; behavioral objective(s); list of materials for lesson; description of teacher behavior and student behavior during the lesson.

- Information Sheets" explain concepts and traffic rules in greater depth for the teacher.

- Worksheets, transparencies, student fact sheets, songs, stories, and lists of films are also provided.

- Ditto pictures tend to be humorous, with few contextural cues for pictures of roads (grass, curbs), with few pictures of road crossing situations, and no minority persons shown.

8. Concepts taught in the following order:

- Existence of safety features in the environment

- Being a courteous pedestrian (this concept is not explained in detail)

- Cross at intersections - follow "Walk," "Don't Walk," and traffic

light signals

- Who safety helpers such as police are

- Name, address and te lephone

- IPOE - Identify hazards, predict alternatives, decide, act 
- Improper pedestrian actions, 1.e., dangers of jaywalking, walking between parked cars, running into street after a ball

- Dangers in bad weather and safety precautions to take

- Important noises in the traffic environment

- Dangers of strangers

- Safety heipers and their duties

- Traffic signs, car and driver signals, traffic lights

- Reasons for pedestrian laws.

9. Pedestrian instructions given:

- Stand on the curb before deciding to cross.

- Look for safety features such as signs, traffic signals, etc.

- Listen and look for approaching vehicles before crossing the street and even while crossing the street.

- Obey "Walk"-"Don't walk" signals.

- Push the button to change the "Don't Walk" light to "Walk."

- Always use sidewalks and crosswalks when available.

- Go directly to home or school.

- Do not run out into the street after a ball.

- Walk on the left side of the street facing traffic when there's no sidewalk.

- Don't accept rides or anything from strangers. Avoid them.

- Obey traffic lights - stop on red, wait on yellow, look each way and go on green.

- Wear white at night, bright clothes in bad weather and dark clothes on snowy days.

- Look both ways for cars (the song doesn't tell children what to look for).

- Don't walk in alleys, dark streets, wooded areas or empty lots.

- Play in safe play areas. 
- Pedestrians have right of way in crosswalks but not at unmarked crosswalks where vehicles have the right of way.

- Walk on the right side of crosswalks.

- Don't hitchhike.

- Obey bridge and railroad signals.

10. Teaching methods/materials used:

- $\quad F i l m$

- Traffic games in simulated environment--some directly related to traffic, others, like "Dog and Bone" game, not

- Discussion

- Teacher role playing in classroom

- Drawing pictures

- Memorization of items (e.g., address and phone number)

- Ditto sheets

- Model of traffic safety features such as "Walk/Don't Walk" signals made by teacher

- Songs

- Three walks in real traffic environment

- Observing traffic situation from school window

- Paper doll and paper clothes

- Tape recorder

- Poem

- Stories such as "Little Red Riding Hood" (for stranger concept), "Once Upon a Corner," etc.

- Models of policemen and traffic signs made by children.

11. Measurement of children's knowledge and behavior:

- None mentioned. 


\section{ILLINOIS}

Name of program: Safety Education Units for Illinois Elementary Schools

1. Authors: Illinois State Board of Education, Illinois Office of Education, Safety Education Section.

2. Sponsors: NHTSA.

3. Date: 1972.

4. Age of children in the program: $K-12$.

5. Kinds of safety taught:

- Pedestrian

- Bicycle

- School Bus

- Car Passenger.

6. Unit reviewed: Kindergarten Leve 1 - Introduction to Pedestrian Safety.

7. Format:

- Fourteen lessons are devoted to pedestrian safety.

- Dittos and songs are integrated into all the lessons.

- Each lesson contains:

-- A description of lesson content

-- Theme (usually define as Regulators, Operators)

-- Learning situation activities

-- Dittos. The dittos are the same as the dittos described in Pedestrian Safety Education for Alabama Youth.

8. Concepts taught in the following order:

- Meaning and use of colors in traffic light and use of bright/white colors in dark and bad weather

- Position and meaning of colors in traffic light 
- Correct pedestrian behavior at traffic light

- Safe work and play areas (for three consecutive lessons)

- Correct pedestrian behavior (rules)

- Spatial position and meaning of colors in traffic light and corresponding appropriate pedestrian behavior

- Meaning of signs and proper and improper pedestrian actions at signs, intersections and traffic lights

- Policemen

- Safety helpers such as safety patrol

- Pedestrian hazards in the neighborhood (driveways, alleys, parking lots)

- Safest route from home to school

- Safety features and pedestrian rules.

9. Pedestrian instructions given:

- Wear white at night, bright colors in bad weather, and dark colors on snowy days.

- Stop on the red traffic light signal, wait on the yellow and look each way (no mention of what to look for) and go on the green traffic light signal.

- Stop, look and listen before you cross the street (no mention of where to stop, what to listen for--no direct mention of curb or sidewa lk).

- Play in safe places.

- Cross the street at crosswalks.

- Cross the street at the corner, not midblock.

- Look to the right, to the left and around the corner behind before crossing.

- Look for approaching cars before crossing the street.

- Walk across streets--don't run.

- Obey police and crossing guards.

- Traffic rules as taught in "I'm No Fool as a Pedestrian," Walt Disney production. 
- Obey stop signs, railroad signs.

- Take the safest route to school.

10. Teaching:methods/materials used:

- Stories about colors, traffic

- Flannel board

- Safety poems

- Games such as "I Spy," "Redlight, greenlight"

- Dittos with pictures of vehicles, a cut-out doll, pedestrian situations, fruit, nursery rhyme stories, traffic light, signs, police and safety diagrams of intersections, helpers, and the "Hazardville, Pennsylvania" ditto.

- Safety songs, weather songs

- Nursery rhymes--Humpty Dumpty, Jack and Jill and 01d Woman Who Lived in a Shoe

- Experiment watching an egg fall and break

- Discussion

- Coloring dittos

- Film

- Drawing pictures

- Model of traffic light, village with safety features

- Three field trips: one field trip on a real street which children are taught to cross with the aid of the model traffic light; another where children walk and look for safety features; another where they observe the speed of cars at intersections

- Making models of traffic signs using collage materials or paint

- Riddles about traffic signs

- Role play

- Memorization of rules

- Discriminating between correct and incorrect pedestrian behavior shown in dittos, flims, etc.

- Maps of children's route from home to school. 
11. Measurement of children's knowledge and behavior

- Designated evaluations (3) which assess children's knowledge through their comments and actions during discussions and role play. There is no evaluation of children's street crossing behavior outside on a real street. 
Name of program: K-6 Elementary Traffic Safety Education Curriculum

1. Primary author: Indiana Department of Public Instruction.

2. Sponsor: NHTSA.

3. Date: 1975 .

4. Age of children in the program: $K-6$.

5. Kind of safety taught:

- Pedestrian

- Bicycle

- Auto Passenger

- School Bus.

6. Unit reviewed: Pedestrian Level I ( $K$ and Grade One).

7. Format:

- The total guide is divided by levels: Level $1(K+$ first); Level 2 (second + third); and Level 3 (fourth - sixth), Curricula for each level is further broken down into the four safety areas cited above. Each safety area section is printed on different colored paper.

- Each lesson is labeled with appropriate numbers and/or alphabet letters which refer to the cross-referencing system which specify academic areas related to the activity. The system is described in a section at the beginning of the curriculum.

- Ditto sheets, which can be reproduced, are included in the back of each grade level section. Almost a third of the dittos are of signs. Each of these dittos has one large sign per page, but no post and no traffic environment context. Merely the top of the sign or traffic light is shown. There are two dittos with realistic drawings of traffic situations. Children are shown walking across the street, calling to a friend across the street and playing on the sidewalk. All children depicted are white middle-class and the girls shown are wearing skirts. Two dittos show "Chucky Clothes Conscious" and clothes for different weather conditions. The last series of dittos focus on perceptual motor activities and concepts such as judging car distance and most direct route to school: The ditto teaching children to judge car distance by city blocks shows a child walking towards three squares on the way to school. Rather than show buildings, etc., to make the blocks resemble city blocks, only geometric squares are used. The dittos on judging car distance again 
substitute geometric squares for city blocks, broken lines indicating crosswalks and only two fully drawn figures--of a boy and a car. The ditto on the most direct route to school shows a curvy path and a straight path from the car to a building. All in all, these dittos are very abstract as few pictures resemble the real objects which they depict.

- A bibliography of resources such as films, books for children, teacher manuals, reference books is included.

- There is an evaluation form to be filled out by the teacher for the purpose of improving the curriculum materials.

- Each of the five chapters in Level One contains the following:

-- behavioral objective

-- background teacher information

-- suggested activities.

N.B. There is one exception. In the "Directionality Chapter" there are no activities--just testing instruments.

8. Concepts taught in the following order:

- Traffic vs. non-traffic related sounds

- Noticing and understanding the meaning of traffic safety features-colors, nine signs, traffic light colors, visible clothing

- Auditory and visual signals which indicate direction of hazards and directions to move to avoid danger

- Safe distance from cars in crossing the street

- Pedestrian rules and guidelines.

9. Pedestrian instructions given:

- Listen for the sounds of vehicles before crossing (no mention of why).

- Look for signs and traffic lights and obey them; these safety features are both vehicles and pedestrians.

- Stop when you see the red light, wait when you see the yellow light and go when you see the green light in the traffic light.

- Stop before crossing the street. (No mention of why, what to look for, etc.l.

- Look right-left-right before crossing the street.

- Walk facing oncoming traffic on the right side of the street.

- Obey directions given by policemen and other safety people. 
10. Teaching methods/materials used:

- Dittos

- Discussion

- Model of traffic light and street with cars; etc.

- Games such as "Red light, Green light"

- Story

- Using colors as symbols in the classroom, e.g., a red tag on scissors means unsafe.

- Flanner board with shapes

- Making signs and other geometric shapes

- Sign puzzle

- Flash cards

- Films recommended

- Sign mobile

- Drawing picturés

- Coloring

- Experiment seeing which colors show up best in a dark room

- Dodge ball

- Most direct route from home to school. A ditto showing two routes a car can take, curvy and straight, to reach a destination, is used to teach this concept.

- Counting blocks (use city blocks as a measure of distance). A ditto showing a boy walking to school past actual square blocks (which look like building blocks) is used to teach this concept.

- Estimating speed/distance of children moving inside the gym

- One visit to the real street to judge car distance and determine when it is safe to cross the street.

- One visit on a blocked-off street to cross the street (with a driver education teacher driving up and down the street)

- One observation walk on a real street to look for safety features. 
- Experiment seeing driver education teacher speed around curb and hit a mannequin that is too close to the edge of the curb.

- Pretending to be on school patrol and pretending to be a pedestrian.

- Songs .

11. Measurement of children's knowledge and behavior:

- The "Directionality Test," a checklist with 22 items, (asking children to do tasks such as "Point to your left foot") is used as a Post-test for Kindergarten and Pre-Test for Grade One. Other directionality tests are paper and pencil tests using dittos which look similar to some Frostig perceptual motor tests. The teacher judges the child's knowledge of directions by observing how he/she draws lines from one picture to another. 


\section{IND IANA}

Name of program: South Bend's traffic safety program for young children

1. Developer: South Bend Police Department.

2. Sponsor: Federal, State, and private funding sources such as local businesses and civic groups.

3. Date: It appears that South Bend has had a long history of safety education sponsored by the South Bend Police Department. Prior to 1975 , much of the teaching of traffic safety to ,children was done by Sergeant Robert W. Miller. In 1975 , following Sergeant Miller's death, South Bend opened their Safetyville, a child-sized town complete with all the regular safety features of a real town--sidewalks, traffic light, signs, etc. The program is similar to but not the same as National Safety Town Center's program; members of the Police Department have developed their own training program and sets of materials for children and parents.

4. Age of children in the program: five to ten-year-olds.

5. Kinds of safety taught:

- Pedestrian

- Btcycle

- Passenger safety.

6. Materials reviewed:

- Coloring book

- Safetyville project brochures

- Safety patrol-brochure

- Safetyville tests for children.

7. Concepts taught/pedestrian instructions given:

- Cross at corners.

- Walk across the street; don't run.

- Obey patrol guards.

- Play in safe places; not near the street.

- Cross the street when the "Walk" light appears. 
- Look both ways before crossing the street.

- Look left-right-left before you cross the street (no mention in book let about what to look forl.

- Obey traffic signals.

- Don't accept rides or anything from strangers.

- Don't run into the street.

- Cross at the traffic light signal.

- Walk between crosswalk lines if they exist; otherwise walk across the street in a straight line.

- Don't play in the grassy section between the sidewalk and the street or on a sidewalk right next to the street.

- Obey traffic guards, school patrol.

- Know the meanings of and obey (6) signs: stop, yield, no bicycles, bike route, school crossing, pedestrian crossing.

- Follow the safest route to school.

- Don't walk or play between parked cars.

9. Teaching methods/materials used:

- Traffic instruction in simulated traffic environment (Safety Town)

- "Safety Suggestions for Parents" brochure which encourages parents to teach traffic safety to children at home and model good traffic behaviors themselves

- Coloring book

- Policeman's discussion with children in classroom.

10. Measurement of children's knowledge and behavior:

- A true or false test on safe/unsafe traffic behaviors is given to children. 
Name of program: Traffic Safety Education for Iowa Schools (K-6) Part II: Pedestrian Safety, School Bus Passenger Safety, Automobile Safety

1. Primary authors: State of Iowa Department of Public Information/ University of Dubuque.

2. Sponsors: Iowa Division of Highway Safety/NHTSA.

3. Date: 1975 .

4. Age of children in program: $K-6$.

5. Kinds of safety taught:

- Pedestrian

- School bus

- Automobile passenger.

6. Unit reviewed:

- Kindergarten Pedestrian Safety Unit.

7. Format:

- There are eight folders in the total curriculum--a general introduction folder and one folder for each grade.

- Each folder has a table of contents for all grades; acknowledgements to the Department of Public Instruction; a foreword: a list of basic safety concepts to master; differently colored sheets for different areas of safety instruction--pedestrian, school bus, automobile and mini-bike safety; cartoon-1ike illustrations; and a bibliography with related books, periodicals, pamphlets and leaflets and audio-visual materials as well as the addresses for the AAA Motor Club of Iowa and National Safety. Council.

- Each page is a lesson and each page contains the following: concept taught; behavioral objective(s); teacher information (general information about pedestrian rules or reason for the lesson objective); activities; interdisciplinary opportunities (relationship of lesson to other academic areas); illustrations (dit to transparency) on the back side of the page. There are eight lessons in the Kindergarten Pedestrian Safety Unit.

- Each of five dittos in the pedestrian safety unit focuses on a different lesson in pedestrian safety. The first requires children to identify pictures of pedestrians. The second focuses on the dangers of construction areas. The third is about visibility in rainy weather. The fourth shows safe and unsafe pedestrian behaviors 
and the fifth is on time to go to school. The first, second, and fourth pictures show sketches of the traffic environment. Not many details are included. For example, there's a boy walking between two cars. Only one cue reveals the fact that one car is parked -- no driver is within. No sidewalk is shown or any other contextual clues to indicate what the boy is doing. Another ditto shows a girl in front of a car. Below the wheels are the letters "screech"--which is not a word that a five-year-old can read. All the characters drawn are cartoon-like, humorous characters. Minority characters are shown and the characters' clothes and their scenes look up-to-date.

8. Concepts taught in this order:

- Meaning of "pedestrian"

- Safe walking on sidewalks (dangers of driveways, alleys, building or street construction, adverse weather)

- Visible clothing

- How to cross a street

- Name and address

- Safest route to school

- Time to go to school

- Accepting a ride only from people parents have approved (beware of strangers).

9. Pedestrian instructions given:

- Try to cross at intersections or crosswalks.

- Wait on the curb, out of the street, while waiting to cross the street.

- If a crosswalk is not controlled, pedestrians must look all ways and cross when the intersection is clear.

- Walk across the street.

- When walking across driveways and alleys, look in all directions for approaching vehicles.

- Keep away from places blocked of for street repair or building construction.

- Wear bright clothes in bad rainy weather.

- Walk the safest route to school.

- Don't accept anything from strangers. 
10. Teaching methods/materials used:

- Discussion

- Dittos

- Experiment (to see visibility of various colors in the dark)

- One field trip to a real street to practice traffic skills

- One session practicing traffic skills in a simulated traffic environment

- Letter to parents

- AAA Safest Route to School unit

- Memorization of rules.

11. Measurement of children's knowledge and behavior:

- Not stated. 


\section{KENTUCKY}

Name of program: Pedestrian Safety Guide K-6 (Revised)

1. Primary authors: Kentucky Department of Education.

2. Sponsors: Kentucky Office of Highway Safety.

3. Date: Original draft from 1966 grant.

4. Age of children in the program: $K-6$.

5. Kind of safety taught:

- Pedestrian

- School Bus

- Personal (name and address)

- Passenger

- Responsibility for Self and 0thers.

6. Unit reviewed: Kindergarten.

7. Format:

- The total curriculum is divided into sections appropriate for each separate grade level. Each section is, in turn, broken down into smaller units which teach different kinds of safety.

- Dittos are integrated into lessons in each unit. The use of dittos does not appear to be emphasized in this Kentucky program as few dittos exist, and as there are many other learning activities suggested. The few dittos in the guide present traffic signs, traffic signal lights, an derial diagram of a bus, a clown and two children who are dubbed the Super Safety Pair. There are no dittos depicting the traffic environment as a whole. For example, the dittos of traffic signs and traffic signals present only the tops of the signals rather than showing them in a traffic environmental context. The dittos of the two children and clown present them in great detail, but no traffic environment.

- One separate page discusses the purpose of having such a curriculum as being to provide a uniform safety program throughout the state of Kentucky and provide a curriculum structure which enables teachers to be more creative in teaching safety concepts.

- The Introduction emphasizes children's need for and right to safety education, and states that the end result of such an education is an individual who possesses the knowledge and skills to make sound rational judgments which in turn, will enable him/her to conduct $\mathrm{him} / \mathrm{her}$ self safely in his/her environment. 
- A safety poem, highlighting a pair of children called the Safety Pair, tells children some of the safety habits the Safety Pair will help them learn.

- Each unit contains the following:

-- Title of safety lesson being taught

-- Overall objective (not behavioral)

-- Behavioral objective

-- "Teacher Information," suggestions about the frequency and manner with which to teach the lesson

-- List of activities

-- oittos.

8. Concepts taught in the following order:

- The meaning of colors of the traffic light.

- The meaning of the following signs: "Rallroad Crossing", "Danger," "Stop," "Walk," "Don't. Walk," and "Wait."

- Traffic sounds and their meanings.

9. Pedestrian instructions given:

- When you come to a traffic light, wait on the yellow signal, stop on red and go on green.

- Obey traffic signs.

- Listen to traffic sounds.

10. Teaching methods/materials used:

- Traffic sorigs

- Coloring dittos

- Games - "Green Light, Red Light"; "I Spy"; "Show Me" (a color);

"Super Safe Says"; "Blindman's Bluff" with voices

- Bullet in board display.

- Field trips (suggested once, but no specific suggestions about where to take children and what to teach them on the field trips)

- Drawing and coloring signs 
- Singing traffic safety songs set to familiar tunes

- Tape with traffic and non-traffic sounds.

11. Measurement of children's knowledge and behavior - None stated. 


\section{LOUISIANA}

Name of program: Safety for Loutsiana Schools

1. Primary authors: Louisiana State Department of Education.

2. Sponsors: Louisiana State Department of Education, Louisiana Highway Safety Commission, NHTSA.

3. Date: 1977.

4. Age of children in the program: $K-9$.

5. Kind of safety taught:

- Pedestrian

- School Bus

- Cycling.

6. Unit reviewed: Pedestrian (K-1 Leve1s).

7. Format:

- The foreword discusses the importance of traffic safety education as a measure for preventing traffic accidents and a way to shape children's basic attitudes at an early age. A traffic safety program integrated into the school curriculum is advocated.

- There is a section which explains the rationale for and construction of the curriculum guide.

- "Pelican Pete", the state bird of Louisiana, is the safety mascot; his story is given.

- There are 14 separate units, each dealing with a different concept in pedestrian safety, in the section on pedestrian safety.

- Each unit contains a heading indicating the type of traffic safety taught; the title of the unit (concept taught); one or two behavioral objectives; a list of traffic safety vocabulary to be taught and definitions (written in language approprlate for adults-for example, "Conflict--an event that.can develop through human error in identifying, predicting, deciding, and executing safe action and can be avoided." p. 35); a section supplying background information to 
the teacher, for example, pedestrian rules and rationale for them; and a series of learning activities suggested for each grade level except kindergarten and first grade, which are most of ten grouped together. In the margin next to each learning activity the relevant grade level and related academic area is shown.

- A set of transparencies (1.e., dittos to be reproduced) is packaged separately, but copies are integrated in each unit for reference. The dittos for the $\mathrm{K}-1$ level generally have few pictures on each page. Pictures of people look like cartoons. Little detail is shown in pictures of people look like cartoons. Little detall is shown in pictures of traffic scenes. For example, straight lines signify roads and sidewalks. In a few pictures squiggles representing grass help the viewer to distinguish the sidewalk from the grassy area. There is an attempt to present children from different racial backgrounds by delineation of hair and eyes rather than color. Girls and women are most often shown wearing skirts or dresses while boys wear pants.

8. Concepts taught in the following order:

- Appropriate pedestrian behavior (meaning of "pedestrian", I.D.P.E. process (Identify, Predict, Decide, Execute)

- Walking on sidewalks and roadways - dangers and safety precautions to take

- Traffic signals, signs and lane markings - their colors, shapes and meanings

- People who assist pedestrians - police, crossing guards, safety patrol, etc.

- Crossing streets and roadways - where to cross the street, how to obey traffic safety features such as signs, correct pedestrian procedures in crossing the street

- Walking to a specific destination (safest route to school)

- Walking alone and in groups (dangers of strangers, personal address and telephone number, and procedure to follow when lost on a class $\operatorname{trip)}$

- Walking under hazardous conditions (dangers of bad weather and nighttime walking; noncultural animals' habits)

- Conflicts and disasters (reasons for auto-pedestrian conflicts); using the I.D.P.E. process during a disaster such as a fire, nuclear attack, etc.

- Playing in neighbor hoods (identifying potential hazards and knowing what to do when an injury occurs) 
- Pedestrians and raflroads

- Holiday and recreational activities involving walking (safety precautions to take during Halloween and other special events)

- When a rider becomes a pedestrian (rules for entering and exiting a vehicle, dangers of accepting rides from strangers).

9. Pedestrian instructions given:

- I.P.D.E. - Identif.y, Predict, Decide, Execute.

- Walk on the left side of the roadway facing traffic coming towards you, if it is necessary to walk on the roadway.

- Don't run out into the street between parked vehicles.

- Walk on sidewalks, not on streets.

- When you're walking on the sidewalk and are about to cross a driveway, check to see if a car is coming out. If so, wait until the car has left the driveway.

- Wear hats and carry umbrellas that do not obstruct your vision.

- Obey safety helpers.

- Look at all cars on the street.

- Be seen before you cross the street; be sure the driver has stopped and seen you. As you cross, look for cars and be sure you are seen.

- Cross at an intersection or at the corner when possible.

- Take the safest route to school, avoiding dangers such as deserted areas, strangers, intoxicated persons, alleys, parked cars, etc.

- Don't talk to or accept anything from strangers; avoid them.

- Wear bright clothes in rain or bad weather.

- If you're involved in an accident, stay calm and remain t the scene. If you see an accident, report it to an adult.

- If your ball rolls into the street, ask an adult for help in getting the bal1. If no adults are present, look in all directions (no mention of what to look for) before getting the ball.

- Cross a railroad at the raflroad crossing.

- Obey ralliroad crossing signals. Do not cross when the light is flashing and the gate comes down. Stop, look, and listen (no mention of what to listen for) before crossing a raliroad crossing. 
- Don't play on rallroad tracks.

- Watch parades and other special events from the curb; don't run after cars or floats.

N.B. Many, many more traffic safety rules are listed in the "Instructional Information" section for the teacher. As it is not clear which of those rules are to be taught to the kindergarten students, only rules directly related to the kindergarten student, only rules directly related to the activities suggested for $K-1$ students have been listed above.

10. Teaching methods/materials used:

- Dittos - coloring and discussing

- Cutting magazine pictures, making a collage

- Discussion

- Drawing pictures

- Interpreting pictures of traffic situations

- Role-play in simulated traffic environment-using tape or chalk on the floor indoors, role-play in a parking lot

- Songs teaching traffic safety, set to familiar tunes

- Experiment seeing how light flashed into the eye affects it

- Tracing models (of signs, etc.)

- Making models

- Games such as "Red light, green light"

- Memorization

- Puzzles

- Walk in a real traffic environment to observe safety features

- Drawing lines to connect dots to make a picture

- Making puppets and using them to role play safety rules

- Visit to classroom by school crossing guard

- Helping to construct bulletin board

- Visit to real street to practice crossing rules with the assistance of the school guard 
- Map of neighborhood

- Riddles

- Story.

11. Measurement of children's knowledge and behavior:

- No formal instruments are shown.

1 


\section{MARYLAND}

Name of program: Safety Instructional System

1. Produced by: The Maryland State Department of Education and Transportation Section.

2. Sponsors: Maryland Department of Transportation (Division of Transportation Safety) and NHTSA.

3. Date: During the 1970s.

4. Age of children in the program: $K-6$.

5. Kind of safety taught:

- Pedestrian

- School Bus

- Bicycle

- Auto Passenger

- School Environment.

6. Units reviewed:

- Pedestrian section of the Kindergarten Unit of Interdisciplinary Traffic Safety Instructional System

- Kindergarten Level Teaching Guide for the Traffic Signal Light Program.

7. Format:

- The Interdisciplinary Traffic Safety Instructional System for the Kindergarten Level is a thick 250-page manual designed to enable teachers to help children develop skills and knowledge in all areas of safety listed above. The emphasis in the pedestrian section is on improving chlldren's visual perceptual motor skills so that they will be better able to master skills needed for pedestrian traffic situations. Thus, while very much related to traffic safety, this is 
more of a general perceptual skills program, teaching chfldren such thing as directions, eye-hand coordination and sound discrimination. The "Prelude" explains the rationale for this approach:
"The survival, safety and success of children depends not so much on knowing a set of rules or regulations about safety, duty by a systematic process of identifying, predicting, deciding and executing a specific behavior when confronted with a potentially dangerous situation.... This process of identifying, predicting, deciding and executing is largely triggered by visual inputs in order to cerebrally match data with stored memory traces that have been also visually: acquired."

- To be used with the manual described ( $p$. iij) above is the Teaching Guide for the Traffic Signal Light Program - Kindergarten Level, a 125-page manual which aids instructors in teaching children about parts of the traffic environment such as sidewalks, crosswalks, intersections, traffic signal light and appropriate pedestrian related behaviors.

- The format of the first manual, Interdisciplinary. Traffic Safety Instructional system, is as follows:

-- "Introduction" ("How To Use This Program' and description of parts of the manual)

-- "Prelude" (rationale for visual perceptual motor program, recognition of people who've contributed to understanding of the role of vision and its relevance to the learning process, list of references on vision)

-- "Safety Instructional System Evaluation" (to be used by the teacher as often as wanted but minimally at the end of each semester)

-- "Safety Film Critique Form" (to be used with films suggested in the bibliography)

-- Table of Contents

-- Units with lessons plans

-- "Subject Area Cross Reference"

-- Bibliography

-- Dittos (masters with directions on back of each of these are integrated into the lessons).

- The format of the individual lessons is as follows:

-- "Objective" 
-- "Concepts To Be Developed"

-- "Expected Competencies"

-- Teacher Information and/or learning activities grouped by subject area

-- Dittos with directions on the back. The dittos primartiy require children to do activities line between two pictures, connect dots, circle the correct response or trace - perceptual activities reminiscent of Frosting perceptual-motor activity sheets. In general, dittos are abstract. For example, the ditto on judging car distance (in terms of city blocks) shows a diagram of a car circling around a city block which is shown as a geometric square rather than a clump of city butldings on a city block. The previous ditto on counting the number of blocks to school also shows blocks as squares, which a young literal child could very well interpret to be bullding blocks and miss the concept entirely. Another example of the level of abstraction is the ditto on distinguishing wide and narrow streets, which shows two parallel lines and two dotted lines (for crosswalks) drawn between the two long lines. Is the child expected to judge widths between four sets of two streets and then transfer this concept to real streets? Some pictures shown are representations of real things and people such as trees, girls, cars and bicycles. Other pictures shown are of basic shapes such as squares, circles, numbers and curvy "s" shapes. The emphasis in this manual on visual perceptual motor skills may be a reason for sticking with strictly perceptual. activities, rather than incorporating more realistic representations of the traffic environment. In genera1, there are few drawings per ditto.

The format of the second manual, Teaching Guide for the TRaffic Stgna 1 Light Program - Kindergarten, is as follows:

-- "Prologue" (information about the traffic light signals to be used as teaching aids)

-- "History of Traffic Controls"

-- "To the Teacher" (information about the kinds of skillis needed in order to obey traffic signal lights)

-- "How to Use the Safety Instructional System Signal Light Program" (acknowledges that the curriculum follows a sequential developmental order and describes how the program materials have been assembled and can be found in the manual)

-- "Signal Light Evaluation Form"

-- Units with lesson plans

-- Suggestions for bulletin boards 
-- "Subject Area Cross Reference"

-- Bibliography.

- The format of individual lessons is as follows:

-- "Objective" (behavioral)

-- "Concept to be Developed"

-- Learning activities described and grouped by subject areas such as music and physical activity

-- Relevant dittos. The dittos are varied, some highly abstract like the dittos described in the first manual, and a few which are more realistic and concrete, as they show some details such as gates, houses, trees and flowers in the traffic environment. However, Most dittos require children to look at a series of two or three pictures of items such as vehicles, whistles or pedestrians, and choose one item which appears larger, different, etc. These dittos appear to be focusing more on visual perceptual tasks than providing representations of the traffic environment.

9. Concepts/skilis taught in the Interdisciplinary Traffic Safety Instructional System in the following order:

- Body image

- Directionality

- Movement behavior (walking or balance beam)

- Eye-and coordination

- Visual discrimination and shape recognition

- Sound discrimination.

- Auditory discrimination (using traffic and non-traffic sounds)

- Distance judgment (cars must be one block away for a person to cross the street safely).

10. Concepts taught in the Safety Instructional System Signal Light Program in the following order:

- What a sidewalk is (where it is, what it's made of); who uses sidewalks; what to do when there are no sidewalks; where pedestrians should walk on sidewalks; the rationale for crossing the street at the corner; sounds heard during walks on sidewalks; locomotor skills

- Purposes of streets; what streets look like; kinds of vehicles on streets; use of the curb; listening and looking for cars 
- Definition and use of crosswalks; different kinds of crosswalks; where to cross the street

- Definition and use of intersections; different kinds of intersections, concept of differences

- Definition of a corner; what to do when one comes to a corner; auditory discrimination; definition of signal traffic light - what it is, how it works

- How to cross the street with the traffic light; meaning of colored signals of the traffic light and appropriate pedestrian actions; the importance of obeying police directing traffic at the traffic light

- That colors can have specific meanings; meaning of red, yellow and green in traffic situations

- Meaning of red, yellow and green in traffic situations, how to read colors of the traffic light; the importance of looking for turning cars at intersections

- Meaning and use of "Walk/Don't Walk" lights; the importance of looking for turning cars at intersections

- Meaning and use of "Walk/Don't Walk" lights; the importance of looking for turning cars regardless of what the "Walk/Don't Walk sign says

- Meaning of the Highway System - that it is made up of physical structures such as sidewalks and cars as well as people; words that are/are not related to traffic; auditory recall; color recognition; math concepts; visual discrimination.

11. Pedestrian instructions given in the Safety Instructional System Signal Light Program:

- Walk on the sidewalk if there is one.

- Walk on the right half of crosswalks.

- When there is no sidewalk, walk on the left shoulder of the highway or roadway facing traffic approaching from the opposite direction.

- Cross streets at the corner, not mid-block.

- Stop, stay behind the curb and look both ways (left and right) for traffic.

- Listen for traffic.

- Use crosswalks when avallable to cross the street. 
- Obey "Walk/Don't Walk" slgns (on the "Walk" signal, check left and right for vehicles and walk if safe. On the "Don't Walk" signal, continue walking if you've already started, but if you're at the curb, do not cross. Always watch for cars while crossing.)

- Walk, don't run between the lines of crosswalks.

- When there's a policeman or policewoman directing traffic at a traffic light, obey him. other and not the traffic light or "Walk" light.

- Don't walk when the yellow and red lights of the traffic light face you, but walk on the green light.

- Look left, right and front before you step off the curb.

- Cars and pedestrians should yield right of way to ambulances, fire trucks or other emergency vehtcles.

12. Teaching methods used in the Safety Instructional System Signal Light Program:

- Role-playing correct pedestrian behaviors in simulated traffic environments (in classroom)

- Discussion

- Fingerplays

- $\quad$ Drawing pictures

- Cutting and pasting

- Making models of traffic safety feature, traffic environment, pedestrians and vehicles

- Games (motor games such as "Hop Scotch", "Mother, May I", jump rope)

- Locomotor exercises (crawling, leaping, walking, moving in different directions) 
- Interpreting pictures of sidewalks

- Dittos

- Songs

- Stories

- Films

- One walk to observe a real traffic situation

- Listening experiences - guessing what makes different kinds of sounds, which items in a story don't fit in

- Examination of a real traffic light

- Experience chart

- Making murals

- Following teacher's directions

- Transparencies and overhead projector

- Making experience books

- Poetry

- Math activities (e.g. one-to-one correspondence activities).

13. Teaching methods/materials used in the Interdisciplinary Traffic Safety Instructional System:

- Use of mirrors

- Motor exercises - body alone and with objects such as balls and balance beams

- Puzzles (of body parts)

- Feltboard (with body parts)

- Discussion

- Games such as "Hokey-Pokey," "Where Is Thumbkin?"

- Fingerplays

- Drawing according to teacher's directions 
- Dittos

- Mimicry activities

- Dramatization through body movement

- Weaving and other eye-hand coordination activities such as stringing beads

- Collage of paper strips - focus on spatiali arrangement

- Matching and discriminating between shapes

- Listening activities

- Imttating traffic sounds

- One visit to the real traffic situation to observe city blocks, watch and judge speed and distance of cars.

14. Measurement of children's knowledge and behavior:

- Several tests are suggested to assess children's perceptual and motor ski11s - a "Body Movement Post-Test for Kindergarten", which can also be used as a Pre-Test for. Grade One; a "Dominant Hand Chart" used by the teacher as the child performs actions specified on the chart; "Oirectionality Pre-Post Tests"; and the "Auditory Perception Test". Teachers can also assess children's skills through their work on dittos and through learning activities. The tests and dittos mentioned are ways teachers measure children's progress when using the Interdisciplinary Traffic Safety Instructional System manual. No tests are suggested in the other manual; most likely teachers assess children's knowledge through their performance in learning activities and their work on dittos. Since both annuals provide behavioral objectives for each lesson, the teacher can assess children's mastery in terms of how well they perform the behaviors listed in the behavioral objectives. In summary, chlldren's general perceptual and motor skills, their knowledge of the traffic environment, their knowledge of traffic rules, and their abllities to execute proper traffic safety behaviors are the primary areas of assessment. It is not specified whether children's adherence to traffic safety procedures is to be observed in actual situations; in fact, it appears that any assessment of their behavior occurs in the classroom, not in the real traffic environment. 


\section{MICHIGAN}

Name of program: Curriculum Guide for Safety Education Grades K-6

1. Primary authors: Michigan Department of Education in Lansing, Michigan.

2. Date: 1973.

3. Age of children in the program: $K-6$.

4. Kind of safety taught:

- Pedestrian

- Bicycle

- School bus

- Automobile passenger

- Pre-Driver Education.

5. Unit reviewed: Pedestrian Safety $K-3$.

6. Format:

- The foreword states that the curriculum seeks to teach children good health and safety habits. It suggests integrating safety education into the teaching of regular academic subjects and utilizing parents and community involvement.

- In the beginning of the guide there is a list of behavioral objectives for children in all safety areas.

- An introduction to traffic safety suggests that teachers teach children traffic safety by setting forth a good example; teaching children traffic safety rules and rationale behind them; helping children understand the relationship between unsafe actions and accidents.

- The K-3 section on pedestrian safety is approximately three and a quarter pages long. There are nine basic lessons in this section. The left column of the page gives the concept to be taught and the right column suggests relevant learning activities, each of which is 
briefly described in a few sentences. At the end of the section on traffic safety, a list of related textbooks and an annotated 1ist of films and filmstrips are given. No dittos are included in the guide.

8. Concepts taught in the following order:

- Personal information such as child's name, telephone and address, and name of school

- Safest route to school, meaning of "pedestrian"

- Going directly to schoor.

- Cooperating with the safety patrol

- Meaning and use of traffic light signal

- Knowledge that some drivers may disobey traffic signals, that cars can be out of control, that drivers cannot stop vehicles instantly

- Meaning and shapes of traffic signs (stop, information, warning, yield, railroad signs)

- Crossing at protected intersections and safety precautions to use when crossing

- Blind spots where the driver cannot see pedestrians - dangers of parked cars and driveways

- Safe behavior on sidewalks.

9. Pedestrian instructions given:

- Take the safest route to school.

- Go directly to school; go directly home.

- Obey the safety helpers.

- Stop on the red signal, wait on the yellow and on the new green traffic light signal, after checking for moving traffic and turning cars.

- Obey traffic signs pertaining to pedestrians.

- Cross streets at protected intersections if possible.

- Use the crosswalk.

- Do not jaywalk. 
- Look all ways to be sure the way is clear before starting to cross (no mention of what to look for).

- Walk, don't run, when crossing the street.

- Don't step out from behind or between parked cars.

- Don't play or stand near a parked car or in a driveway.

- Use the sidewalk - walk on the right.

- Look for cars backing out of driveways and coming out of alleys.

- Don't chase others, throw balls or wrestle on the sidewalk (1.e., don't play there).

- Remove objects that may cause someone to fall on the sidewalk.

- Be extra careful during bad weather and poor light.

- Be prepared for unexpected hazards such as strangers, threatening dogs or bullies.

10. Teaching methods/materials used:

- Role-play

- Many walks into the real traffic environment to observe pedestrtan behavior, traffic safety features, names of streets and to practice crossing the street

- AAA safest route to school

- Orawing and labeling pictures

- Writing stories about consequences of unsafe pedestrian behavior

- Visit to classroom by a safety helper such as the school patrol

- Flashcards testing knowledge of traffic safety features

- Model of traffic environment

- Making models of signs

- Discussion

- Experiment noting how hard it is just for people to come to a sudden stop 
- Experiment sitting in a real car (parked in driveway or school playground) to discover blind spots

- Panel discussion or quiz show reviewing safety rules and practices

- Crayoning

- Reading books about safe walking and traffic safety.

- Visit by Blue Birds or Camp Fire Girls who explain their traffic safety project.

11. Measurement of chlldren's knowledge and behavior

- No instruments provided or suggested. 


\section{MICHIGAN}

Name of program: Preprimary Traffic Safety Curriculum.

1. Primary authors: Michigan Department of Education.

2. Sponsors: State of Michigan Office of Highway Safety Planning and NHTSA.

3. Data: 1978-1981.

4. Age of children in the program: four and five-year olds.

5. Kind of safety taught:

- Pedestrian

- School bus

- Tricycle/bicycle

- Passenger.

6. Unit reviewed:

- None, as materials were being developed and pilot-tested during the time of this ilterature review. All information pertaining to this effort was gained through a telephone interview.

7. Format of program:

- There is a parent component to the program. Plans include a filmstrip to motivate parents to teach their children traffic safety education.

- For children, two storybook activities, such as hand puppets and finger plays, and experiences improving children's fine and gross motor skills will be developed.

- The program will be implemented in a preschool program which has a parent component. 
8. Concepts taught/pedestrian instructions given:

- Cross the street only at a safe location.

- Cross the street only with an adult.

- Demonstrate where and how to walk when near the street.

- Demonstrate that the curb is an invisible stop sign.

- Listen for approaching cars.

- Watch for cars by looking al1 ways and by finger-scanning before crossing the street.

- Recognize vehtcles backing up in driveways.

- Don't cross a driveway, if a driver is in the car in the driveway, unt 11 the driver either moves the car or gets out of the car.

- Demonstrate a knowledge of strangers and how to avoid them.

- Recognize the common traffic signs.

- Recognize hazardous pedestrian situations.

- Develop habits of conduct that will enable children to meet traffic situations with as little danger as possible to themselves and other." N.B. objectives listed above are quoted from Dr. Ann Johnson, Traffic Education Consultant on the project.

9. Teaching methods/materials used:

- Storybook

- Parent teaching child in real traffic situations

- Puppets

- Finger Plays

- Discussion resulting from open-ended questions.

N.B. Other methods may be used in this program which are not listed above.

10. Measurement of children's knowledge and behavior:

- There will be a pilot test of the program. Interviews will be conducted with parents of children in the program to find out what gains they perceive their children to be making as a result of the program. Teachers will also observe parents' and children's behavior in traffic. 
11. Evaluation of this traffic safety program:

- Pilot test described above. 
MINNESOTA

Name of program: Minnesota Traffic Safety Curriculum Guide $(K-3)$

1. Primary authors: Department of Public Safety/Department of Education in the State of Minnesota.

2. Sponsors: Minnesota Department of Public Safety and NHTSA.

3. Date: 1973.

4. Age of chlldren in the program: $K-3$.

5. Kind of safety taught:

- Pedestrian

- School Bus

- Car Passenger.

6. Unit reviewed: Kindergarten-pedestrian sections.

7. Format:

- The preface describes some accidents of elementary school children in Minnesota and emphasizes the important role that teachers play in teaching children safety habits which may prevent such accidents.

- Eight of the twenty-two lessons in the kindergarten unit are concerned with pedestrian safety. These lessons are not grouped together; rather, they are interspersed with lessons on school bus, car passenger and bicycle safety.

- Each lesson contains: a "Theme" description (kind of safety education); a "Topic" (overall lesson concept); "Subject Application" (related academic area); "Suggested Time" (to teach the lesson); "Lesson objective" (general objective - not behavioral); "Background" (information-for the teacher-about students' needs, the rationale for traffic safety rules or additional information about rules and behavior); "Suggested Activities"; dittos which are incorporated into the lessons.

- The dittos included in the curriculum guide depict urban, suburban, and rural traffic environments in a realistic manner. There are no dittos showing chlidren scanning for vehicles or deciding whether to 
cross the street, but there is one ditto of two boys crossing at an intersection and a boy restraining a girl from chasing a ball into the street. Several dittos show children waiting for, boarding,a and riding on a bus. The dittos showing children transmit a real feeling of vitality and movement. the children depicted are from a variety of racial and social backgrounds, and their appearance is very up-to-date. The remaining dittos in the series are more abstract than the ones already described. One is essentially a diagram or map of streets and city blocks (for the safest route to school) and another is of the top of the traffic light and series of fruit and vegetables (to teach the spatial positions of read, yellow and greenlights on the traffic light.)

8. Concepts taught in the following order:

- What is "safety"

- Reason for traffic rules

- Meaning of "pedestrian"

- Safest route to school (which includes many concepts such as safety features and dangers of railroad crossings)

- Proper use of stdewalks and roadways

- Correct way to cross the street

- Emergencies - knowing where to find help and how to avoid trouble with strangers and animals

- Safety helpers - police, school patrol or crossing guard

- Traffic signal lights

- Safe places to play outdoors in the winter

- Safe places to play outdoors in the summer

- Walking at night or in bad weather.

9. Pedestrian instructions given:

- When you walk, watch for cars.

- Take the safest route to school: avoid ratlroad crossings, uncontrolled intersections, and construction areas, but waik on routes where there are sidewalks, crossing guards, etc.

- Don't run into the street after anything (such as a ball).

- Wait for the bus on the sidewalk or roadway shoulder. 
- Use sidewalks whenever they are avallable: When there are none, walk on the left side of the roadway facing traffic.

- Don't trespass on private property.

- Cross the street at intersections with traffic light signals and on crosswalks if possible. Cross at intersections, even if they are not controlled, rather than midblock.

- If you cross the street midblock, you must yield right of way to vehicles.

- Don't accept anything from or talk to strangers.

- Stop, look and listen before you cross the street la song that does not specify to look and listen for cars).

- Stop at the red signal light, wait on the yellow and look each way then go on the green signal of the traffic light.

- Play in safe areas--in back yards, parks, etc.

- At night wear light bright colors (yellow and white) and wear contrasting bright colors such as. red and green in snow storms.

10. Teaching methods/materials used:

- Discussion

- Experiment seeing how well retroreflective tape can be seen in a dark room

- Collage of magazine and newspaper pictures

- Story

- Memorization of rules

- Games such as: "Simon Says," "Red Light, Green Light"

- Poetry

- Visit of policeman to classroom

- Maps

- Orawing a mural

- Dittos with illustrations to color

- Film 
- Role-play using a simulated street, sidewalk and rallroad crossing in classroom

- Songs

- Poem

- Request parents walk children on safest route to school.

11. Measurement of children's knowledge and behavior:

- None suggested. 


\section{MINNESOTA}

Name of Program: Godfrey Gopher Coloring Book

1. Sponsor: Minnesota Department of Public Safety.

2. Date: Unknown

3. Age of children for whom the coloring book is intended: $k-3$, most likely.

4. Kind of safety taught:

- Pedestrian

- Car passenger

- $\quad$ Sledding

- Bicycle

- School bus.

5. Sections reviewed: Those pertaining to pedestrian safety.

6. Format:

- Pages in the coloring book are loose rather than stapled into the book.

- Godfrey the Gopher, the safety mascot, appears in all pictures and tells children (seen in a variety of traffic situations in the pictures) the approprtate safety rules to follow. Often a short reason for each rule is given after the role is stated. For example, "Don't play behind a car in the drive. It may backup and you won't be alive!" Sometimes ideas are given tolfit the rhyme--at the expense of content. "When you wait for the bus, stay off the road. Don't hop around like a big, brown toad." The tone throughout is a bit humorous.

- There is one fllustration per page and usually one safety rule fingle at the bottom of the page.

- Children shown in the 1 liustrations are representative of different ractal and ethnic backgrounds. Both girlis and boys are shown breaking traffic rules or in difficult traffic situations. They are wearing a wide varlety of appare 1; girls wear pants and overalls as well as dresses. Clearly, there is an effort made to make the illustrations up-to-date, non-sexist, and multi-racial.

- Traffic environments shown in the pictures are, for the most part, realistically drawn with contextual clues such as trees, grass, etc. to help children to identify parts of the traffic environment that are being represented. 
- While coloring is the activity called for on most pages, there are a few pages each with a dot-to-dot number game to be completed.

7. Concepts/Pedestrian instructions taught:

- When your ball rolls into the street, don't chase it. Instead, look (no mention of what to look for verbally, although the picture shows an approaching car) and then move your feet.

- Don't play on railroad tracks.

- Look both ways and at the cross-street (no mention that this should be done when crossing the street and no mention made of looking for cars).

- Don't play behind a car in the driveway.

- Don't hitch your sled up to a car for a ride.

- Don't walk between parked cars. The reason for this is stated, "If a driver can't see you when you start to cross, he may hit you hard and give you a toss!" The picture shows a girl walking between parked cars.

- Don't play ball in the street.

- Stop on the red light signal, wait on the yellow, and go on the green.

- When you walt for the bus, don't wait in the street. Stand in the road.

- If there are no sidewalks, walk single file on the left side of the street.

- When walki 


\section{MISSOURI}

Name of program: Missouri PK-3 Safety Curriculum Resource Guide

1. Authors: Missouri Safety Center at Central Missouri State University at Warrensburg.

2. Sponsors: the Missouri State Department of Elementary and Secondary Education.

3. Date: Unknown - now out of print.

4. Age of children in the program: pre-kindergarten - grade 3 .

5. Kind of safety taught:

- Pedestrian

- Playground

- Rural

- School

- Seasonal

- Vacation

- Traffic

- Tricycle and bicycle

- Emergency preparedness

- Fire

- First aid.

6. Unit reviewed: A11 sections concerned with pedestrian safety.

7. Format:

- The introduction explains that this is a guide which integrates all areas of safety (cited above) into academic areas.

- The main units in the curriculum guide are as follows: Art; Language Arts; Math; Music; Science; Physical Activities; and Social Studies.

- Under each unit heading (art, etc.), there are sections on each of the safety curriculum areas (such as pedestrian safety). Often pedestrian safety activities were found under topics such as traffic safety. 
- In general, each unit consists of descriptions of activities with no objectives, materials needed for activities, time allotted or any other regular curriculum feature listed separately from the activities. Sometimes activities stated were really objectives.

- There are very few suggestions for teaching the activities to children of different developmental levels.

- Occasionally resources (films, books, etc.) are mentioned in a unit.

- Many of the same activities--the "Hazardville Pennsylvania" ditto, "Scotty the Scarecrow" safety mascot story--used in other state curricula are included in this curriculum.

8. Concepts taught:

- Colors in signs and traffic lights

- Crossing at the corner (where to cross but not how)

- Traffic lights

- Sixteen signs - shape and meaning. (Though all signs are introduced earlier in the unit, the teacher is finally told on $p .77$ that kindergarten chlldren are only expected to learn 6 signs.)

- Crossing the street (only at the corner)

- Time to leave for school

- Looking both ways before crossing a street

- How to use a crosswalk

- Safe areas to play

- Number of traffic signs on route to school

- Numbers of traffic lights on route to school

- How to estimate car speed and distance

- Proper sidewalk behavior

- Safety helpers--policemen.

9. Teaching methods/materials used:

- Fijms 
- Filmstrips

- Stories

- Memorization of rules

- Discussion

- Models of traffic lights

- Some field trips but no guide as to what objectives to focus on or what to do or look for or how to teach on the trips

- Role play

- Dittos

- Policeman teaches children to use the crosswalk

- Nursery rhymes and poems

- Role play a mock traffic court situation

- Games such as safety BINGo.

10. Measurement of children's knowledge and behavior:

- Children are asked to use items listed on a self-evaluation ditto form to rate their own pedestrian behavior. 


\section{NEBRASKA}

Name of program: Nebraska Pedestrian and Bicycle Safety Curriculum Guide (K-6)

1. Authors: Fred E. Tichenor and Duane H. Schmidt.

2. Sponsors: Nebraska Department of Education.

3. Date: Unknown

4. Age of children in the program: $K-6$.

5. Kind of safety taught:

- Pedestrian

- Bicycle.

6. Unit reviewed:

- Kindergarten Pedestrian Unit.

7. Overall format includes:

- "Foreword" (reason and need for this program)

- "Introduction"

- "How To Use This Guide"

- "To The Teacher" (short section explaining that a number of factors cause traffic accidents)

- "Traffic Safety K-6 Aims" (general goals)

- "Specific Objectives in Elementary Traffic Safety Education"

- "Children's Growth and Development" (very general statements concerning how children learn and process traffic safety information; for example, it states that five and six-year-olds learn safety through dramatic play).

- "Rules of the Road" (summarizes laws by which pedestrians must abide for $2-1 / 2$ pages).

- "Reference Sheet for Laws" (simplified summary of pedestrian laws along with 5 most common reasons for pedestrian traffic accidents: jay walking; playing in the street; walking on the wrong side of the road; wearing dark clothes when walking in traffic at night; waiting in the street for cars to pass rather than on the curb.) 
- Resource and materials list for units

- Dittos: Generally, there are one or a few pictures on each ditto. Some illustrations have 1 ittie detail and offer few clues to the real object depicted. There are no children from minority groups shown and clothes depicted are out of date. (Women are always wearing skirts, etc.l.

- Format of individual lessons in the kindergarten unit contains:

-- Content (kind of safety taught)

-- Level (grade level)

-- Concept

-- Resources and materials such as dittos are not listed, but included at the end of the lessons on pedestrian safety.

-- Learning activities (5-10 per unit) are described.

8. Concepts taught in this order:

- Development of attitudes, values and judgments

- Safest route to school (AAA Safest Route to School Program)

- Personar facts

-- knowing name, phone, address

-- evaluating play areas (play in streets)

-- being lost

-- what "pedestrian" means

- Learning the traffic light colors and spatial positions of colors on the traffic light

- Traffic signs and controls

-- signs and meanings

-- "Walk-Don't Walk" signals

-- push-button pedestrian control

- Pedestrian actions

-- crossing at corner

-- walking not running.

- looking first

-- traffic light colors

- Basic pedestrian rules

-- crossing at corner

-- sidewalk - no sidewalk

-- special hazards 
- Pedestrian clothing

-- weather conditions

-- being seen

-- night time

- Safety helpers

-- police

-. safety patrol

- Special problems and dangers in pedestrian safety

a) crossing driveways and alleys - parking lots

b) leaving between obstructions

c) dangers with animals

d) strangers

e) railroads

f) pedestrian-vehicle transfer areas (boarding bus)

g) pedestrian sidewalk vehicles

h) holiday dangers (Christmas and Halloween)

- Responsibility and respect for rules and laws.

9. Teaching methods/materials used:

- Discussion

- $\quad$ AaA unit

- A few field trips

- Models of traffic light and signs

- Flannel board stories

- Songs

- Dittos

- Role-play in classroom

- Memorizing rules

- Games such as "Red Light, Green Light"

- Stories

- Films

- Children's drawings 
- Poems

- Darkening classroom (visibility experiment)

- Collages of safety helpers.

10. Measurement of children's knowledge and behavior:

- Evaluated children's knowledge primarily through their work on dittos and comments during discussion. The "Pre-Test Evaluation" for grade I can be used as a Post-Test for kindergarten. Two items on the test --demonstrating how to cross the street at unguarded and guarded intersections--focus on children's actual behavior in traffic. 
Name of program: Professional Guide K-3 Traffic Safety Resource Curriculum State of Nevada

1. Primary author: John Hawkins.

2. Sponsor: State of Nevada Governor's Highway Safety Program/NHTSA.

3. Date: 1977-1979.

4. Age of children in the program: $K-3$.

5. Kinds of safety taught:

- Pedestrian

- Bicycle

- School bus

- Automobile passenger.

6. Unit reviewed:

- Pedestrian K-3 Unit.

7. Format:

- The introduction to the entire K-3 curriculum defines the purpose of safety education and sets up guidelines for teaching safety education: "regularly scheduled safety instruction," "Integrating safety into regular curriculum subjects," "correlating safety activities with other learning experiences," and capitalizing on the "teachable moment" when children are discussing a traffic accident or dangerous traffic situation in casual conversation. (quotes from $p$. 15-16). Additional guidelines suggest that teachers should determine the sequence of material to be taught; choose which activities are most appropriate for their children; make use of local resource personnel to assist in their safety education program; involve students' parents in the program; and remember that "local school district and local law enforcement procedures, rules and regulations take precedence over any recommendations made in this material." ( $p$. 20)

- The introduction to the pedestrian safety unit presents facts about child pedestrian accidents and lists the following as possible reasons for the accidents: chlldren's height as impeding visibility, 
children's undeveloped perceptual abilities, their limited reasoning abflities, short attention spans, egocentrism, lack of understanding of the meaning of death and danger, their intensely emotional and spontaneous reactions and impulsiveness, and their tendency to express themselves through actions rather than words. The conclusion drawn is that children need both protection and guidance (instruction).

- Two checklists or pre-test instruments, one for kindergarten teachers and one for teachers of grades 1-3, are provided to enable teachers to determine their students' knowledge and needs.

- Fourteen lessons are included in the pedestrian section. Each lesson includes the following: 1isting of subject, unit and concept to be taught; behavioral objectives for the lesson; grade levels the lesson is appropriate for (sometimes a lesson is appropriate for K-3, sometimes just $1-3$, or fust $K$ ); 11st of materials; descriptions of what children should know and do and of what the teacher should do; and dittos. Often there are additional sections in the lessons which provide teachers with more background information, additional activities, and suggestions about which concepts to emphasize. Several pre and post tests are also provided for teachers and parents to assess children's knowledge and behavior.

- There are several kinds of dittos in this guide--cartoons of people, a few realistic drawings of traffic environments, drawings of signs, some dittos focusing on teaching directions. Although there are a few dittos which show children on recognizable sidewalks (one cintld being approached by a stranger and the other playing on the sidewalk), there are no dittos in the pedestrian section which really depict urban, suburban or rural traffic environments. Several pictures of children dressed in approprlate wearing apparel for snowy and rainy days are included, but none of these dittos depict children in the traffic environment. The children depicted appear Caucasian and middle class; no minorities are shown. There is a picture and word story designed to teach children how to cross the street, but there are no pictures of children practicing these skills in a real traffic environment. There are many dittos of traffic signs (or the tops of traffic signs) but no environmental context. One ditto teaching the concepts of city blocks depicts an overhead map of streets and blocks with houses drawn on them. Several dittos focus on teaching and/or assessing directions. These dittos have pictures of geometric shapes, figures, and objects and require children to circle or underiline representations located in one particular place. Most of the dittos have one or two pictures per ditto; the pictures are large and easy to see.

8. Concepts taught in the following order:

- Personal information (addresses, etc.) 
- Directionality (spatial concepts such as left, right, in front, and in back as wel1 as motor coordination and perception are emphasized)

- Looking for and recognizing traffic signs and lights (stop, yield, one way, pedestrian, rallroad)

- Rules and courtesies of a pedestrian (waiting on the curb to cross.) (Many many skills are taught in this lesson from noting rear view mirrors to dangers of walking between parked cars to what to do at an unguarded intersection to walking on the left side of the road when no sidewalk exists.)

- Hazards and precautions to take at night, at dusk and during bad weather - appropriate clothing to wear, etc.

- The traffic light - meaning and spatial position of each color

- Identification of traffic signs

- Strangers - not to talk to or accept anything from strangers and not to go in areas where strangers may lurk

- Safe pedestrian routes.

9. Pedestrian instructions given:

- Cross the street at corners.

- Obey traffic signs (e.g., Ped $x$-ing).

- Pedestrians have right of way in crosswalks only, but pedestrians should not cross until all traffic has completely stopped or until they can safely reach the other curb.

- At a traffic light, cross only on green light facing you, stop on red and wait on yellow.

- Always look and listen before crossing and check to the left around the corner before crossing (because of right on red).

- Stay at least one foot behind the curb until the light signals "go" or "walk" and traffic clears. If there is no light, wait unt1l all traffic clears, look all directions and watch for turning cars before crossing.

- Walk to the right in the crosswalks, and stay between the lines.

- Push the button on a pedestrian operated signal and only cross after the signal says to "go" and you've checked for cars from all directions. 
- Don't cross the street or walk between parked cars.

- Walk on the left shoulder of the roadway facing traffic when there is no sidewalk.

- Obey safety officers, school guards, etc,

- When coming to an intersection, stop, observe the street, check for moving and turning cars, and listen for traffic.

- Walk across the street; don't run.

- Cross at right angles, not diagonally.

- Beware of drivers--they do not always stop.

- Cross within pedestrian crossings.

- Take the safest route to school.

- Always walk on the sidewalk when possible and walk on the right of the sidewalk.

- Wear bright colored clothes during bad weather and white or reflective materlal at night.

- Don't accept rides or anything: from strangers.

10. Teaching methods/materials used:

\section{- Dittos}

- Games such as "Red Light", "I Spy"

- Tape recorder

- Role play

- Writing activity

- Models of safety features such as signs and the traffic light made by teacher

- Flannelboard

- Experiments with objects to determine such things as visual perception

- Explanation by teacher

- Practicing skills on a real street 
- Observation of a real car

- Observation walk on a real street

- Viewing traffic from school window

- Children making models

- Discussion

- Song

- Puzzle

- Poem

- Model of traffic environment made by class

- Charts, e.g., listing reasons for accidents

- Creating safety symbols and using then

- Bulletin board on safety current events

- $\quad$ AAA safest route to school.

11. Measurement of children's knowledge and behavior:

- Pre-test for kindergarten teachers to assess student's knowledge

- Post-test directionality test

- Parent/Teacher Pedestrian Observation Chart

- True/false pedestrian safety test for students

- Knowledge test on 10 items (e.g., "I always cross the street at corners center of the block"). 
NEW YORK

Name of program: Iraffic and Pedestrian Education Systems (TAPES)

1. Authors: Elisar Research Corporation (under contract).

2. Sponsors: New York State Department of Motor Vehicles/NHTSA.

3. Date: 1969-1971.

4. Age of children in program: four to six years of age.

5. Kind of safety taught:

- Pedestrian

- Bus

- Car passenger

- Bus .

6. Program materials reviewed:

- TAPES Module I

- Traffic and Pedestrian Education System - A Report

- $\quad$ TAPES Leader's Guide.

7. Format:

- Fifteen modules with flimstrip and cassette tape presentation and many related activities to be taught during a 15-week period

- A teacher's guide (Leader's Guide) which contains script cues, module outlines, songs and games

- A set of 23 ditto masters--activity sheets that include artwork and games which reinforce concepts presented in the filmstrips

- Modules taught:

- Introduction

-- Judging Car speed

-- Stop! Go!

-- The Yellow Traffic Light 
-. Walk! Don't Walk!

-- A Safe Walk

-- Crossing Streets

-- Crosswalks and Intersections

- What Cars Do

-- Trains and Tracks

- A Ride in a Car

-- Playing Safely (Part I)

-- Playing Safely (Part II)

-- Riding the School Bus

-- Walking to School or Bus Stop

- Format of the Leader's Guide

-- Introduction (which discusses safety concepts to be covered in the module)

-- Materials required for the module

-- Script cues for audiovisual presentation

-- Reinforcement activities such as songs, games, discussion, dittos

-- Supplement reinforcement activities (to be used during the following week and integrated with other programs).

8. Concepts taught:

- The reasons for learning about traffic

- The meaning of key traffic-related terms

- The meaning of key road markings, certain traffic signs, and policeman's signals

- Where and how to walk safely

- Precautions to observe in bad weather and at night

- How to cross the street, aided and unaided

- How to behave near rallroad tracks

- How to behave on a school bus -

How to behave in the car

- Basic bicycle riding rules -

Where to play and where not to play

- How to pick a safe route to school or to the bus stop. 
8. Teaching methods/materials used:

- Flimstrip using a cartoonlike format with characters who display safe and unsafe pedestrian behavior, foremost among whom is Super sidney the Supersaferoo, a fantasy character with four eyes, legs which leap, muscles made of stee 1

- Songs

- Games

- Dittos

- 8 out of 80 activities involve instruction on real streets

- Games simulating outdoor traffic situation

- Collages

- Role play

- Stories

- Making models.

9. Measurement of children's knowledge and behavfor:

- Children's knowledge was tested after completion of the program by using the "Traffic and Pedestrian Education System Pictorial Traffic Safety Quiz," a twenty question quiz (based on the behavioral objectives in the program's modules) that requires students to circle the correct picture in a series of two on three pictures to answer the question posed by the tester. The quiz was devised by Elisar Research Corporation. For further information: Traffic and Pedestrian Education System: A Report. C. W. Bostick, D.J. McNaughton, and D. L. Hughes, New York State Department of Motor Vehicles; November, 1975.

10. Evaluation of this traffic safety curriculum:

- Parents and teachers of 5,942 children taught the TAPES program were sent questionnalres to find out the adults' perceptions of the program's effectiveness. Most of the parents (80\%) felt that their children had increased awareness of traffic safety, and $83 \%$ of these parents believed that this awareness was in part due to the program. A majority of teachers reported that the program helped children develop better attitudes toward safe pedestrian practices and safer behavior. The teachers (94\%) also reported that they felt the TAPES program to be effective. 
- The Traffic and Pedestrian Education System report cited above states that in 1975 a statistical analys is of accident records of geographical areas where children had been exposed to the TAPES program was in the process of being conducted and would be avallable at a future date.

- The report cited above states that "children exposed to the TAPES program exhibited greater knowledge about pedestrian safety than similar children not exposed to the TAPES program." (p. 33) The mean scores on the experimental groups were significantly higher than the mean scores in the matched control groups $(P .01)$. Oniy children in city school districts were given the quiz. For additional information about the quiz and quiz results see the Traffic and Pedestrian Education System - A Report cited above. 
Name of program: K-g Traffic Safety Resource Curriculum

1. Primary authors: Research Triangle Institute.

2. Sponsors: North Carolina's Governor's Highway Safety. Program and NHTSA.

3. Date: June 1975.

4. Age of children in the program: $K-9$.

5. Kinds of safety taught:

- Pedestrian

- Bicycle

- School bus

- Passenger

- The focus is on increasing children's knowledge of safety rules and the traffic environment.

6. Unit reviewed:

- Level $A(K-1)$ Pedestrian Safety.

7. Format:

- The introduction presents general pedestrian accident facts concerning children, a general description of types and causes of children's pedestrian accidents and major unit objectives.

- The Pedestrian Safety Unit checklist is included to help teachers assess student's knowledge and needs.

- Each lesson provides behavioral objectives, rationale for lesson concepts, list of activities.

- There's a resource list of traffic safety organizations, media, books, and magazines for both students. and teachers, additional games, transparencies, posters, songs, and records. 
- There are several different types of dittos included in this guide. First of all, there's a series of dittos on traffic signs and traffic signals. These depict the top of the sign or signal light only; no traffic environment context is provided so that the child can see where the signs belong. The signs are large and easy to see as there's usually one sign per page. There are several dittos which do present the traffic environment. These dittos show children crossing the street at intersections and walking on sidewalks. The traffic environments portrayed in these dittos are realistically drawn with several detalls such as grass, trees, gates, bulldings, etc. that provide contextual clues enabling a viewer to easily differentiate sidewalks from streets. The traffic environments all have sidewalks but it is not totally clear whether these are inner city or suburban environments. No rural traffic environments are shown. Another series of dittos focus on teaching children directions. Children are expected to compare, circle, and underline a series of objects shown. Another series of dittos focus on teaching children about appropriate clothing for various weather conditions. "Chucky Clothes Conscious," who appears in so many curriculums, appears here. He's a cut-out doll with clothes to be cut out and put on him. There are no dittos depicting visibility problems during bad weather or in darkness and no dittos showing children in traffic during such occasions. The last series of dittos, which teach the most direct route to school and the city block concept, are very abstract. The most direct route to school is shown by a straight path versus a curving path; there is no attempt to illustrate the concept in terms of a real traffic environment. The same holds for the dittos showing city blocks which present an abstract aerial view of city blocks (using rectangles to represent city blocks) and a side view of figures walking past the blocks. These few dittos are the most abstract in the curriculum guide.

8. Concepts taught in the following order:

- Personal information - child's name and address, etc.

- Listening for traffic noises

- Meanings of colors and shapes used in the traffic environment (traffic light colors, signs, colors for night and bad weather visibility)

- Directions (including right and left)

- Judging distance-speed-time (child is taught it's safe to cross when a vehicle is one block away)

- Pedestrian responsibilities lists all pedestrian traffic rules and related information such as what school safety patrol and crossing guards are). 
9. Pedestrian instructions given:

- Look and listen for cars.

- Pedestrians have the right of way in crosswalks only; yield to cars if you are not in a crosswalk.

- At traffic 1ights, cross only at the green light, stop on red, wait on yellow, always look and listen before crossing on the green light, and look behind around the corner before crossing.

- It's safe to cross the street when a car is one block away or further.

- Obey safety officers, crossing guards etc.

- Walk, don't run across the street.

- At intersections stop, look, listen and cross--if there are no cars closer than one block away or if the cars at the intersection stop completely.

- Don't cross on a green turn arrow.

- Walk right across at right angles, not diagonally.

- Walk on the left facing traffic when no sidewalk exists--on the ground adjacent to the road, not on the road itself.

- Do not step into the road from between parked cars.

- Walk on the sidewalk--on the right.

- Wear white or reflective material on your clothes during bad weather or at night.

- Don't hitchike.

10. Teaching methods/materials used:

- Discussion

- Reasons for rules are emphasized

- Memorization of traffic rules

- Role play

- Dittos - coloring, following directions, analyzing pictures

- Games such as "I Spy," obstacle courses 
- Tape recording traffic sounds

- Models of traffic light, signs, street

- Stories

- Drawing pictures

- Mobiles of signs

- Poems

- Songs

- Two observation walks in the real traffic environment.

11. Measurement of children's knowledge and behavior

- A checklist to guide the teacher in assessing students' needs

- A pre-and post-test to assess student's knowledge of directions.

12. Evaluation of this traffic safety curriculum

- University of North Carolina's Highway Safety Research Center at Chape 1 Hill evaluated children's knowledge and behavior before and after the curriculum was taught. From knowledge tests and behavioral observations of a sample of students in grades three, six, and nine, it was concluded that there was a statistically significant increase in knowledge in grades three and six, but not in grade nine. There was no evidence of improvement in children's pedestrian behavior. The Highway Safety Research Center recommendations include revising the curriculum so as to include some focus on behavioral practice of traffic safety principles being taught and involving additional personnel such as crosswalk guards to reinforce traffic safety concepts taught in the classroom. 
NORTH. DAKOTA

Name of program: The Governor's Highway Safety Coloring Book

1. Producers: Department of Public Instruction and the North Dakota Highway Department in Bismark. North Dakota in cooperation with the National Highway Traffic Safety Administration.

2. Date: Unknown

3. Age of children appropriate for: $K-3$.

4. Kind of safety taught:

- Pedestrian

- Car passenger

- Bicycle.

5. Unit reviewed: $A 11$.

6. Format:

- This 15-page coloring book has one illustration per page of a boy or girl either role playing or actually dealing with a traffic situation. Above the child is a safety caption. The same two children depicted throughout look almost identical; there is no attempt to show minorities or children who are different. The clothes the children wear are somewhat dated - the girl in a little dress, the boy in pants. Some lllustrations provide enough contextual clues to aid a young child in discriminating between sidewalk and road. Others provide few details. For example, it's difficult to tell whether the girl in the wagon is on the road or sidewalk. The caption on one page tells children to use the crosswalk, but shows no plcture of a crosswalk. In other words, it is assumed that "crosswalk" is part of the K-third grade child's vocabulary.

- On the back cover of the coloring book is a "P.S. for Mom and Dad." The four points emphastzed to parents are:

-- that parents can help their children know and obey simple safety rules

-- that parents can supervise their chlidren so that they take the safest route when they leave home

-- that parents should set a good example when they drive the car

-- that parents as taxpayers and citizens can support good safety education programs in the community: 
7. Concepts taught in the following order:

- Where to cross the street (at a traffic light)

- Wearing a safety belt

- That it's safer to walk on a crosswalk

- Wearing bright clothing at night

- That cars should be checked for safety

- Meaning and use of stop sign

- Meaning of traffic light colors

- Safe places to play

- What to do if the ball rolls out into the street

- That bicyclists should use hand signals

- To play extra carefully when you're not feeling well

- Not to run into the street, especially between parked cars

- The responsibility of teaching others safety.

8. Pedestrian instructions given:

- Cross the street at a traffic light.

- Use the crosswalk.

- When walking outside at night, wear bright clothing.

- Stop when a stop sign faces you (no mention of where to stop), look for cars and "other things in the street", and if nothing is in the street that could hurt you, walk across the street.

- Stop on the red traffic signal light, use caution on yellow and go on the green.

- Find a safe place to play.

- When the ball rolls out into the street, look both ways before going after the ball (no mention of what to look for).

- Don't run into the street, especially between parked cars. 
9. Teaching methods/materials used:

- Coloring

- Encouraging parents to teach their children safety rules, set a good example as drivers, make sure children walk the safest routes, and support safety education programs in the community

- Discussion. It is probably hoped that parents will read the captions on each page to their children and discuss the concepts and rules taught.

10. Measurement of children's knowledge and behavior:

- None stated. 
OHIO

Name of program: Bicycle/Pedestrian Safety Education: Curriculum Guide

1. Developers: Ohio Department of Education and Ohio Traffic Safety Education Center.

2. Sponsors: Ohio Department of Transportation/NHTSA.

3. Date: 1976.

4. Age of children in the program: Elementary school children.

5. Kind of safety taught:

- Pedestrian

- Bicycles

- Schoor Bus.

6. Units reviewed:

- Pedestrian.

7. Format:

- The 53-page pedestrian section contains three basic sections after the "Table of Contents" and "Introduction" (which gives basic facts regarding chlld pedestrian accidents and suggestions for the curriculum guide): "Safe Walking Practices"; "Safety Signs and Signals"; and "Walking Under Special Conditions." Each of these sections has the following format:

-- Tasks (general instructional objectives)

-- Performance objectives (behavioral objectives)

-- Reference Materials (printed materials, films and filmstrips)

-- Content (information for the teacher on pedestrian safety rules and rationale for those rules)

-- Learning activities

-- Worksheets and/or posters.

- No learning activities or performance objectives or traffic rules in the content area are classified by grade or developmental level; teachers are expected to choose concepts and activities that are appropriate to their students. No guidelines are given. 
- The posters portray various traffic environments with a lot of real1sm and a medtum amount of deta11, more detall than the usual ditto. The streets in the posters, for example are darkened so that there is a clear difference between sidewalk and street. Grass, shrubs, and buildings bordering the sidewalks help to show sidewalks as distinct entities. Several posters have one large panorama covering the entire page, showing, for example, a city intersection with cars, a city intersection without cars and a railroad crossing. The rest of the posters have two illustrations per page, some of which show a total traffic scene such as the walking path, town in the background, railroad tracks and rallroad signal light and gate. The other posters show only a small section of the traffic environment, a part of the road and sidewalk and hedge, for example. These posters typically focus on contrasting behaviors--children walking in the street vs. children walking on the sidewalk, for instance. The pedestrians shown in the fllustrations are wearing up-to-date and varied wearing apparel. Children of different ethnic and racial backgrounds are shown. Girls are shown breaking as many traffic rules as boys; clearly there is an attempt to be non-sexist in portraying characters. The figures' actions are easy to interpret, partiy because a contrast technique is used on the posters with two pictures per page.

8. Concepts taught through the learning activities in the following order:

- Personal information (name and address)

- Where to walk when there's no sidewalk

- Different traffic sounds

- Safest route to school

- How to judge the speed of approaching cars and cross the street

- How to cross at intersections

- Meaning of traffic signs and signals and appropriate pedestrian behavior.

- Safe and unsafe pedestrian behaviors in the following situations: walking when there is no sidewalk; where to walk and play when there is a sidewalk; crossing the street at a crosswalk; where to play when railroad tracks are near; what to do when coming to a ratiroad crossing where the signal is flashing and gate is down; where to walk when there is a sidewalk; what to do at an intersection; which part of the sidewalk on which to walk; where to cross the street; what to do while crossing the street; what to do at raliroad tracks and at intersections with and without traffic.

- Meanings of signs and signals 
- Oriver's responsibilities to pedestrians

- Dangers of darting out into the street

- Meanings of traffic words--"intersection," "rałlroad, "automobile," "parking lot," "parked," and "traffic"

- The time it takes for moving things and people to stop

- How to tell when a vehicle is going to turn

- The dangers of parked cars--how both pedestrians and drivers are affected, blind spots in the car

- Scanning and listening for traffic--how to do it and why it's important

- Pedestrians and driver's attitudes as affecting their behavior

- Safe distances or gaps for crossing the street

- Roles of safety helpers such as police and importance of pedestrians obeying safety helpers

- How to cross a street

- Meanings and correct use of signs--their shapes, colors, symbols, and printed information

- Consequences of incorrect pedestrian behaviors

- Difficulties of seeing in the dark--the need and ways to increase visibility

- What to do to increase visibility during bad weather

- How clothing can hinder a pedestrian's visibility

- That children think differently, attend to different things when playing and when being pedestrians

- Safe praces to play

- Games that are particularly dangerous to play near the street because they bring players into the street

- Use of underpasses and overpasses

- Dangers of walking on wet surfaces

- The importance of good visibility at intersections--that some have bad visibility. 
N.B. The concepts listed above are derived from examination of the learning activities and given in the order of the learning activities. Concepts presented under the section outlining performance objectives sometimes vary slightly from concepts presented in the learning activities. For example a performance objective given on page 45 is "The student will identify those special precautions that pedestrians must follow when walking near railroad crossings," but there is no activity which corresponds. However, there is a direct correspondence between the "Performance objectives" and "Content" sections of the guide. (The "Content" section provides background information--traffic rules and rationale for traffic rules--for the teacher and the information contained therein is intended for the children.)

9. Pedestrian instructions given:

- Whenever there is a sidewalk, walk on the sidewalk.

- When there is no sidewalk, walk on the left side of the road facing oncoming traffic. Walk single file.

- Whenever possible, use controlled intersections.

- Don't cross midblock.

- Obey traffic signals.

- When crossing the street at a light signal, check in all directions before stepping off the curb. Watch for turning cars.

- Wait on the curb while waiting for a traffic light to change or waiting to cross an intersection.

- At intersections with no signs or 1ights, watch for oncoming cars from all directions and look carefully for cars that might be hidden by a building or some other obstruction.

- Treat drfveways and alleys like streets; check to see if cars are backing out or turning in.

- Use pedestrian paths, overpasses, underpasses and safety is lands whenever possible.

- Don't ever cross from between parked caris.

- When crossing at an intersection, walk, don't run and walk in a straight line. Don't loiter.

- Don't walk on freeways, expressways or controlled-access highways.

- Obey safety helpers such as police, crossing guards and school safety patrol. 
Obey traffic signs.

On the red traffic light signal stop. On the yellow, use caution, clear the intersection, and don't start across the street. On green, search for cars and if there are none, cross whtle looking for cars. (There is no mention of the need to read the traffic light signal colors facing you. Clearly the language and ideas in the content section are written for adults and intended to be interpreted and reworded for chlldren.)

- At a flashing red light stop and cross the street only when the road is clear enough to do so safely.

- At a flashing yellow 1ight, stop, check in both directions, and move on ly when the way is clear.

- At the "Walk" signal cross while looking for turning cars. Be cautious even though you, the pedestrian, have the right-of-way. on a "Don't Walk" signa1, complete crossing the street if you are already halfway across-don't run, however.

- At night don't look directly into the oncoming car's headilights.

- Wear light-colored clothing, carry a flashlight and wear light reflective patterns when waiking at night.

- Don't walk along a roadway at night unless it is an emergency. Move away from the travel path when you see the headlights of a car and you're walking on the road at night.

- Be extra careful when walking in bad weather. Be careful that your clothes don't obscure your vision and that your clothes are bright-colored.

- Obey all signals at raflroad crossings. Check both ways before crossing each track.

- Don't run across railroad tracks.

- Don't play on rallroad tracks.

- At Halloween wear a light color, reflective tape, and makeup rather than masks. Don't play in the streets.

- When walking on underpasses or overpasses, walk on the right in single flie with no running or fooling around. Don't climb on or lean on the protective rafilings.

- Never try to cross or walk along a freeway.

Don't approach strange animals and stay clear of strangers. Report strangers to the police and run to the nearest house for help. 
10. Teaching methods/materials used:

- Preparing an address book

- Discussion

- Obstacle course

- Games such as directions games, "Simon says" and traffic-related card games

- Posters or dittos and worksheets

- Pasting footprints on ditto masters of intersections

- Listening activities with vision blocked

- Six trips to real traffic environments to make observations

- "My School Route Survey"

- Map of each child's safest route to school

- Letter to parents, requesting them to chart their children's safest routes to school

- Practice estimating speed and distance of cars and practice crossing an area using a simulated traffic environment (tape. or chalk to represent streets and balls to represent cars)

- Filims

- Two sessions practicing traffic safety behaviors in real traffic environments

- Volunteers modeling correct street crossing behaviors

- Poster contest

- Writing safety memo to parents

- Word chart and other kinds of charts

- Experiment seeing how long it takes runners, walkers, bicyclists, and hopping students to come to a complete stop

- Examination of real car in parking lot

- Mathematical problems estimating amounts of time for vehicles to travel certain distances

- Visit to classroom by safety helpers 
- Constructing models of traffic safety features such as signs

- Flash cards with signs and traffic signals on them

- Experiment flashing light into student's eyes in a darkened room

- Designing something white to be worn at night

- Flannel board activities

- Race getting dressed in clothes appropriate for specified weather conditions

- Learning station

- Show and tell with clothes that are good for walking in bad weather

- Interpreting photograph of real traffic environment

- Experiment seeing how sidewalk surface changes when wet by water

- Taking photographs or drawing pictures of real traffic situations

- Collecting newspaper stories or pictures of winter accidents.

11. Measurement of children's knowledge and behavior:

- No measurement instruments are provided or suggested. 


\section{OKLAHOMA}

Name of program: Oklahoma Curriculum Guide and Student Handbook for Teaching Pedestrian Safety Education K-3 (Scotty the Scarecrow)

1. Prepared by: The State Committee on Pedestrian Safety Education of the Ok lahoma Curriculum Improvement Commission, OK lahoma Highway Safety Program, Ok lahoma State Department of Education.

3. Date: 1973.

4. Age of children in the program: $K-3$.

5. Kind of safety taught:

- Pedestrian

- School Building

- Bus

- Vision Screening

- Oisaster Preparedness.

- Field trips.

5. Units reviewed: all.

6. Format:

- "A Message from the Governor of Oklahoma" (rationale for curriculum guide)

- "Forward" (general goals of the curriculum, suggestions for using the curriculum in an interdisciplinary fashion)

- Introductory statement from the Oklahoma Highway Safety Program

- Section on ways to develop a community based pedestrian safety program

- "The Teacher's Role in the Pedestrian Safety Program" (Objectives, Method of Approach, Suggested Activities)

- Centerfold of colorful Scotty the Scarecrow and directions for making Scotty puppets. There are suggests of how to introduce Scotty and the program, a fantasy story about Scotty coming to life and the meaning of the Safety colors in his costume. 
- The guide is arranged by months, beginning in August and ending in May. The program is sequential and geared to meet seasonal needs.

- At the end of the guide are the following sections:

-- safety songs

- film ilist

-- order blanks for fllms

-- list of sources of materlals and services.

- Each lesson contains:

-- Titie of lesson

-- Objectives (instructional not behavioral)

-- Background information for the teacher when appropriate

-- Method of approach (more specific account of which concepts should be emphasized and what should be expected of the children)

-- Suggested activities (separated by grade levels)

-- Suggested film

-- Suggested bulletin board activities

-. Dittos.

- The dittos, interspersed throughout the curriculum, are not very detalled in terms of portraying the traffic environment. Intersections in the dittos, for example, look as if they were taken directly from a driver's education course; they are diagrams. In another illustration of a child crossing mid-block the lack of detail for items such as grass, sidewalk, street boundaries, etc. makes it difficult--even for an adult--to decide whether the figure is on the sidewalk. The dittos have one or only a few items per page to make it easier for children to "read" the illustrations. Items such as signs are usually shown without their posts and rarely are they shown in context of a real situation. The persons depicted are all Caucasian and their wearing apparel is not up-to-date. Certain stereotypes such as that of a teacher always being female and police always being male are reinforced through the illustrations.

7. Concepts taught in the following order:

- Meaning of "pedestrian"

- Who Scotty the Scarecrow is

- Meanings of safety colors put in signs

- Personal information (name, address, etc.)

- Parts of Scotty the Scarecrow

- Community helpers to contact in an emergency 
- Dangers of strangers

- Meaning and use of the School Zone, School Crossing, Pedestrian Crossing, Stop, Raflroad, Yield, Warning and "Walk/Don't Walk" signs, meaning and correct pedestrian procedure for traffic signal light colors

- Meaning and use of railroad signs and crossing gates

- How to cross at intersections of various types (with and without crosswalks, intersections with four lanes)

- That cars will not stop at intersections without crosswalks and signs

- Dangers of crossing driveways

- Dangers of clowning around and playing in the street

- Dangers of disobeying traffic light signals

- Dangers of not watching traffic

- Dangers of walking between parked cars

- Dangers of chasing balls into the streets

- Dangers of jaywalking

- Dangers of petting strange animals

- Dangers of walking outside at night

- Meaning of "pedestrian," "crosswalk, "traffic light, " and "curb"

- Correct way to walk on a crosswalk

- Role of the Safety Patrol, crossing guard and policeman

- What constitutes the safest route to school.

- Use of underpasses and overpasses

- Dangers of bad winter weather

- Dangers of carrying sharp objects

- What to do, whom to go to in an emergency

- Safe places. to play (playground)

- Safe procedures for walking to and from a playground 
- Dangerous places to play (construction sites, quarries, vacant lots, rubbish piles, electrical lines)

- Meanings of signs used to warn people of construction sites

- Dangers of walking in the dark and ways to make pedestrians more visible

- Dangers of walking in the rain.

8. Pedestrian instructions given:

- Go to community helpers when you're in trouble.

- Don't walk or play alone; stay with friends.

- Don't accept rides or gifts from strangers. If a stranger approaches you, run away.

- Don't go near strange dogs.

- Obey traffic signs.

- Stop on the red traffic light signal, wait on the yellow and look each way and go on the green when cars have stopped.

- Push the button to change the traffic light.

- Stop, look and listen before crossing ralliroad tracks.

- Stay at least one foot behind the curb until traffic clears or the light signals you to go.

- Look in all directions and watch for turning cars before crossing an intersection.

- Walk on the right in crosswalks and stay between the lines.

- Don't crawl between railroad cars or boarding trains.

- Don't throw objects at trains or put objects on the rajlroad tracks.

- Look a11 directions before crossing the street (no mention of what to look forl.

- Don't cross busy streets and intersections with islands in them by yourself.

- Don't play or stand near a parked car in a driveway.

- Don't clown around or play in the street. 
- Cross only at corners and in crosswalks.

- Walk across intersections--don't run or hor seplay.

- Don't jaywalk or enter the street from between parked cars.

- Don't chase a ball into the street.

- Wear white or carry a light when walking at night.

- Obey safety helpers such as crossing guards, safety patrol and police.

- Take the safest route to school. (most direct and safest route with a minimum number and length of street crossings, where there aren't complicated intersections and dense traffic, where safety helpers are always on duty, where chlldren converge at one place to cross the street, where streets have control signs and/or signals).

- When there is no sidewalk, walk facing traffic on the left side of the roadway.

- Take overpasses and underpasses when available.

- Walk on the right and don't run, stop or horseplay in underpasses and overpasses.

- Don't play in the snow near the street or sled near the street.

- Take extra care wher walking out in the snow.

- Report accidents and/or emergencies to parents and adults at school.

- Walk into the playground area.

- Play ball and other games in a place where objects such as the ball won't go into the street; don't play in or near the street. play in playgrounds, backyards, and area away from the street.

- If your toy goes into the street, report it to the teacher on playground duty. Don't go after the ball without permission.

- Don't play near construction sites, quarries, vacant lots, rubbish piles, electrical lines, roads or ditches.

- Walk on the right of the sidewalk.

- Wear prescription glasses if you need them.

- Wear bright colors in bad weather.

N.B. Approximately five of the above traffic rules are given in the "Method of Approach" section but are not dealt with in "Suggested Activities" for kindergarten students. 
9. Teaching methods/materials used:

- Stories about traffic safety, Little Red Riding Hood

- Discussion

- Drawings

- Memorization

- Visits to the classroom by safety helpers

- $\quad$ One field trip

- Dittos--coloring and following directions

- Role play in simulated traffic environment (in classroom)

- Songs

- Poems, Chants

- Puzzles

- Model of traffic signal light, city, underpass, etc.

- Games such as fishing for traffic signs

- Flannel board activities

- Puppets

- Films

- Map of home to school route

- Illustrations of traffic environment

- Collage of magazine pictures

- Chart on safe and unsafe places to play

- Experiment seeing the visibility of different colors in a darkened room

- Bulletin board activities.

10. Measurement of children's knowledge and behavior:

- No instruments are given. The teacher may be expected to assess children's knowledge through their work on dittos and conversation. 
OREGON

Name of program: Traffic Safety Education for Oregon Schools $(K-3)$

1. Sponsors: Oregon Department of Education.

2. Date: Unknown, probably later than 1974.

3. Age of chlldren in the program: $K-3$.

4. Units most closely reviewed: Kindergarten.

5. Format of individual lessons in units:

- "Area": (social responsibility)

- "Sub-area" (citizen streets and highways)

- "Program goal" (one of the following goals listed below):

1) "Demonstrate an understanding of the use of color to maximize the safety of the pedestrian."

2) "Demonstrate an understanding of proper use of sidewalks and roadways."

3) "Demonstrate knowledge of the proper precautions to take to minimize the possibility of molestation."

4) "Demonstrate an understanding of the rules of the road for bicycle operation."

5) "Demonstrate the proper technique for operating a bicycle safely."

6) "Demonstrate an understanding of the ways to maximize the safety of all school bus passengers."

7) "Demonstrate a general understanding of the dangers of riding in or on a motor vehicle."

8) "Demonstrate an understanding of the protection afforded by passenger restraint equipment."

- "Competency" (Course Goal).

- "Suggested Application" (to related academic areas).

- "Performance indicator" (behavioral objectives)

- Materials to be used

- Learning situation activities

- Related activities. 
6. Concepts taught:

- Red, yellow and green colors

- Meanings of above colors in traffic situations

- Pedestrian visibility at night and during bad weather conditions

- Spatial placement of colors on traffic light

- Proper pedestrian behavtor on sidewalks

- Proper pedestrian behavior crossing roads (this varies slightly according to the poem or rule suggested in the learning activities)

- Meanings of traffic signs (stop, ratiroad crossing, school crossing signs)

- Roles of police and safety patrol

- Dangers in traffic situations

- Name and address

- Safest route to school

- Dangers of strangers.

7. Teaching methods/materials used:

- Games

- Poems

- Songs

- Weather chart and dress-up doll

- Flannel board activities

- Modern stories/fairy tales

- Dittos

- Role play in simulated traffic environments

- Making models

- Forming a safety club 
- Film

- Field trip to real streets

- Game from National Safety Council

- Coloring/drawing activities.

8. Measurement of children's knowledge and behavior:

- Rarely suggested. Knowledge may be measured through use of dittos and teacher observation of children doing learning activities. 


\section{PENNSYLVANIA}

Name of program: A Teaching Guide for Child Pedestrian Traffic Safety

\section{Primary authors/sponsors: Commonwealth of Pennsylvania}

Department of Public Instruction.

2. Date: 1965.

3. Age of children in the program: $K-3$.

4. Kind of safety taught:

- Pedestrían.

5. Material reviewed: The entire booklet.

6. Format:

- An accompanying book let Pedestrian Safety: A Resource Unit, also prepared by the Commonwealth of Pennsylvania Department of Public Instruction in 1967, supplies the teaching methods used to teach concepts presented in a Teaching Guide for Child Pedestrian Traffic Safety. These two publications, plus the Walt Disney Study Prints and the Pennsylvania edition of the Pedestrian Safety Set, comprise the total program materials.

- Pedestrian Safety: A Resource Unit provides lists of activities to use with the K-3 age range. No clarification of which activities are best suited to the different grade levels is provided. The activities are generally divided into introductory learning and culminating activities. Each activity is described in a sentence or two. No behavioral objectives, rationales for the activities, list of materials, or detailed descriptions of the learning activities are provided. At the back of the book let are lists of books for children, books for teachers, films, filmstrips, pamphlets and book lets, magazines for teachers, records, songs, plays, posters and pictures, circulars and leaflets, transparencies, and games.

- The Teaching Guide for Child Pedestrian Traffic Safety, an 11-page guide, is very colorful and easy to read, laid out with few illustrations and paragraphs per page. The centerfold is the famous "Hazardville, Pennsylvania" illustrated activity showing pedestrians and drivers breaking a multitude of traffic safety rules, an activity that has been reproduced in a number of curricula from other states. The "Introduction" in this guide gives an overview of contents given in the guide. The first page informs teachers that the dart-out accident is children's "greatest killer." The rest of the book consists of safety rules to teach children in order to prevent dart-out behaviors. All illustrations are of urban traffic environments. The cartoonlike illustrations in the book, while colorful, are somewhat dated. All characters shown are wearing 
slightly dated clothes--females in skirts or dresses, and males in pants. There is no variety of races or ethnic groups depicted; all characters are white with straight blond or brown hair.

7. Concepts taught in the-following order:

- Consequences of a pedestrian darting out into traffic from between parked cars

- Short cuts in traffic can be dangerous

- Parked cars restrict both the driver's and pedestrian's visibility

- Vehicle's difficulties of making sudden stops

- Places where drivers expect pedestrians to be/not to be

- Meaning and use of crosswalks

- Looking all ways before crossing the street

- Cooperating with safety helpers such as police, crossing guards, safety patrol

- Meaning and use of traffic safety features such as the stop sign, traffic light, "Walk/Don't Walk," and yield, school signs

- Safe places to play

- Student's difficulty in concentrating on both play and traffic

- Playing safely all year long

- Dangers of accepting. rides from strangers

- Where to walk when there are no sidewalks

- Nighttime visibility measures that pedestrians should take

- Dangers of bad weather.

- Safe routes--what constitutes a safe route.

8. Pedestrian instructions given:

- Don't dart out into the street from behind parked cars.

-. Avoid dangerous short cuts.

- Use intersections with control safeguards where available.

- Cross at intersections. 
- Use crosswalks.

- Cooperate with safety helpers.

- Before crossing at an intersection with a stop sign, wait and make sure there is no traffic before crossing.

- On the green traffic light signal, wait until you are sure all cars are giving you the right of way.

- Watch for vehicles and don't leave the curb if one is coming, even in areas where signs tell drivers to slow down or yield.

- Don't play in the road.

- Don't accept rides from strangers.

- Walk on the left side of the road facing traffic whenever there are no sidewalks.

- Wear white after dark.

- Avoid busy or complicated intersections.

- Use crossings with maximum visibility and the fewest obstructions.

- Take the most direct and safest route to your destination.

9. Teaching methods/materials used:

- Discussion

- Visit by State Police and dogs, local police, safety patrol

- Film/filmstrip on pedestrian safety

- Record related to pedestrian safety

- Book on story related to pedestrian safety

- Newspaper items on pedestrian dangers

- Review of TV and radio reports on safe or unsafe pedestrian practices

- Construction of signs

- Making a list of safety words and definitions

- Role play of various traffic situations

- Making models of clay figures and objects to create a traffic environment and dramatize safety practices 
- Puppet show

- Creation of stories and poems

- Flannel board

- Drawings

- Writing safety rhymes

- Singing songs

- Games such as "Bingo" to review safety vocabulary and "Red Light/Green Light"

- Tour of school grounds and neighborhood

- Making a safety booklet

- Map of community

- "Safetyville" and Hazardville"

- Illustrated book of safety rules

- Children's testimonies of pedestrian experiences

- Practicing safe pedestrian behaviors in driveways near the school

- Experiment seeing colors of materials and types of materials which reflect the most light in a dark box

- Experiment seeing which colored clothes show up best in a darkened room.

- Safety newspaper

- Tracings of children!s feet as a reminder to walk safely

- Panel discussion

- Six visits to observe the real traffic environment

- Study prints (from Walt Disney) display.

10. Measurement of children's knowledge and behaviö:

- It is suggested that some of the learning activities be used for evaluation. In addition, simple tests, self-analysis inventories, rating scales and observational techniques to be used during the learning activities are suggested. No specific instruments are suggested. 


\section{RHODE ISLAND}

Name of program: Color Your Streets Safe

1. Prepared by: Rhode Is land Governor's Office on Highway Safety.

2. Date: Unknown

3. Age of children for whom booklet is intended:

- Preschool-E lementary.

4. Kind of safety taught:

- Pedestrian

- Car passenger

- School bus

- Bicycle.

5. Material reviewed: All.

6. Format:

- This 14 page $8-1 / 4^{\prime \prime} \times 5-1 / 4^{\prime \prime}$ booklet has one fllustration per page with one or two safety rules written at the top of the page. Most of the pages begin with the big heading "Do"; only two pages begin with "Don't."

- There is an attempt to show an Afro-American child with the White American children and a child wearing glasses, another minority. Most children look like cartoon characters with smiles on their faces. Their wearing apparel is slightly out of date, with all girls wearing short little dresses, and the only woman shown wearing a short dress and heels. The traffic environment provides several details--including an indication that drivers are wearing seat belts, a shaded page with moon and stars to indicate nighttime and, in many cases, curbs, signs rooted on posts in the ground, and fences or grass to indicate the boundary of the sidewalk. However, details such as the cars for which children standing on the curb are looking and the boundaries of the street are usually not shown. The center picture shows two children standing on opposite sides of the street with a crosswalk covered up by a huge display of signs, a missed opportunity to show a whole view of an intersection. A criticism of the pictures of the traffic environment in general is that too little of the traffic situation is shown. Adults have a mental image of the elements that are missing--the cars for which the children are searching, for example--but children may not have yet formed images of each element of the traffic scene. The environments shown have sidewalks and other indications that these might be urban and suburban traffic environments. There were no rural traffic environments shown. 
7. Concepts taught/pedestrian instructions given in the following order:

- Use crosswalks and sidewalks.

- Obey the crossing guard.

- Obey traffic signals.

- Look both ways before crossing (no mention of what to look for).

- Be alert when walking into the street (noimention of what to be alert for).

- Know the meaning of the following signs and obey them: Stop, 55 Speed Limit, Yield, Pedestrian Crossing, One Way, No Bicycles, School, Walk/Don't Walk.

- Wear white after dark.

- Don't ever play in the streets.

- Never run between parked cars.

- Always walk facing traffic (no mention of why, although no sidewalk is shown).

8. Teaching methods/materials used:

- Coloring pictures

- Discussion with an adult, teacher or parent, is suggested in the back cover letter from J. Joseph Garraky, the Governor of Rhode Island.

9. Measurement of children's knowledge and behavior:

- No assessment instruments are provided or suggested. 
UTAH

Name of program: Elementary Safety Education for Utah Schools (Unit 1 , Pedestrian Safety $K-3$ )

1. Sponsors: The Utah State Board of Education.

2. Date: 1974 .

3. Age of children in the program: $K-3$.

4. Format:

- The foreword (the need for and importance of traffic safety education)

- An introductory lesson, Scotty the Scarecrow and traffic colors

- Units for $K$, Levels $1,2,3$

- Dittos are integrated into all lessons. Generally, one or only a few pictures are on a ditto page. No minority children are shown. All females wear skirts (not modern wearing apparel.) Some pictures have many realistic details. A few are very sketchy and abstract. In general, dittos in this guide are very similar to those in the Alabama and Illinois curriculum materials.

- List of supplemental materials.

5. Kind of safety taught: Pedestrian.

6. Units reviewed: Kindergarten.

7. Format of individual units:

- Content

- Theme

- Objective (behavioral)

- Suggested application (to other academic subjects)

- Materials

- Learning situation activities.

8. Concepts taught:

- Traffic light colors

- The weather

- Appropriate clothes to wear for visibility 
- Traffic lights-spatial orientation and meaning of colors

- Safe work and play areas

- Correct pedestrian procedures at traffic lights

- Shapes and meanings of signs

- Rules for crossing at intersections

- Policemen - safety helpers

- Safety patrol helpers

- Safe routes to and from school

- Safe pedestrian behavior.

9. Teaching methods/materials used:

- Stories

- Fłtms

- Flannel board activities

- Charts

- Dittos

- Songs

- Games

- Nursery rhymes

- Role play

- Slides of real traffic lights

- Riddles

- Poems

- Models - of a village, street

- Two field trips to see real traffic situations.

10. Measurement of children's knowledge and behavior:

- No instruments are provided or suggested. 


\section{VERMONT}

Name of program: Safety Is Forever: A Comic Book About Safety

1. Primary author: Jon Woodard.

2. Sponsors: Vermont Agency of Transportation Highway Safety Program/NHTSA.

3. Date: 1978.

4. Age of children for whom the book is intended: School age $(K-6)$.

5. Kind of safety taught:

- Pedestrian

- Car passenger.

6. Material reviewed: All.

7. Format:

- In this six-page comic book with black and white cartoons, children are introduced to Calvin the Safety Cat, a mountain lion dressed up in a policeman's uniform who teaches two children one safety rule after another.

- The characters depicted in the cartoons are Caucasian and always dressed in traditional fashion, with the boy wearing pants and the girl wearing a big bow in her hair and skirt; thus, there has been no attempt to depict minorities or portray children in up-to-date attire.

- There are an average of seven comic pictures per page, interspersed with drawings of the traffic light signal and various signs. The overall effect of all the pictures is busy and cluttered.

- The language used varies from short simple words to abstract words such as "visibility," "extremely," "automobiles, "familiar," "caution" and "shove." It is assumed that children are familiar with terms such as "intersections" and "crosswalks, "for these terms are not explained.

- The traffic environments shown are rural and urban. These environments are depicted in a very realistic fashion with grass, telephone poles, shading of streets, etc., to make separate parts of the traffic environment easy to differentiate. The dittos focusing on walking in the dark and in bad weather show children in the complete environmental context so that visibility problems are more apparent to the viewer. 
8. Concepts taught/pedestrian instructions given in the following order:

- Don't play near the street.

- Don't talk to strangers.

- Safety is forever.

- On a road with no sidewalk, walk on the left facing traffic.

- At intersections make sure the driver sees you (no mention of making sure the driver has come to a complete stop).

- Look both ways when you cross the street (no mention of which way to look first).

- Stop when the red traffic signal light is facing you, caution on the yellow and go. on the green (no mention of looking in all directions, but general advice to be carefull.

- Obey "Walk/Don't Walk."

- Meanings of colors and shapes of the following kinds of signs:

-- hill

-- stop

-. school crossing

-- railroad.

- Don't play in the driveway.

- Never run into the road from between parked cars.

- Be alert for when a train is coming: the gate goes down; bells ring; lights blink; the train whistles; and the train appears.

- Never cross a railroad track when the signal lights are blinking.

- Look both ways before crossing the railroad tracks.

- Play in a playground away from busy streets and highways.

- Wear white when walking: along the road at night.

- Wear seat belts in the car.

- Be careful when walking during bad weather, especially when cars are turning right on red. 
9. Teaching methods/materials used:

- Comic format to entertain and interest children in traffic safety

- Use of a fantasy figure (catamount) to promote interest in traffic safety

- Reiteration of traffic safety rules

- Illustrations

- Coloring the fllustration of the traffic light signal and signs.

10. Measurement of children's knowledge and behavior:

- None stated. 


\section{VIRGINIA}

Name of program: Wi11 Walkwright Programs

1. Sponsors: Highway Safety Division of the State of Virginis/NHTSA.

2. Date: 1976 - to present.

3. Age of children in the target audience: Grades K-4.

4. Kinds of programs:

- A 30-minute film presentation focusing on pedestrian, bicycle, car, school bus and skateboard safety. is shown on TV or on $16 \mathrm{~mm}$. film. The film has an introductory segment with a host (George Douglas) who introduces the program, a drama about the famous pedestrian, private-eye. Will Walkwright, who introduces traffic rules into the town of Careless Corners and makes it a safer place for pedestrians, and a 6-question quiz so that children watching the program can see how much they have learned about pedestrian safety.

- A brochure called "Will Walkwright's Rules for Happy Walking," a four-page spread giving a True-false pedestrian rules quiz (and answers) and suggestions for parents, grandparents and older friends on other rules to teach the children.

- The Will Walkwright Highway Safety Club Handbook, a 10-page booklet full of safety activities, a Safety Club badge and membership card. The activities include:

-- Matching pictures of traffic safety measures and symbols

-- A game "What's wrong with this picture?" showing people breaking traffic rules (answers are given upside down)

-- A maze - "Safety First Come Home"

-- Uncle George's Read Aloud Story," about a boy bicyclist who didn't obey traffic rules but learned to be careful

-- A connect-the-dots game which emphasizes the need for safety belts when riding in a car

-- A request to children to send in their ideas about traffic safety to the Virginia Highway Safety Division

-- A Will Walkwright Highway Safety Club badge and membership card.

- The Will Walkwright Safety Stuff Contest for children in Virginia. Children entered their own stories, posters, poems, plays and videotape. Each child received a free Safety Club T-shirt iron-on for participating, and one child won the contest.

5. Concepts taught:

- Use of sidewalks/use of streets

- Where to walk when there's no sidewalk 
- Safe play areas

- The meaning and use of traffic lights

- Walking rather than running across the street

- Wearing white/reflective materials at night for visibility

- Meaning of "Walk/Don't Walk" light signals

- Meaning and use of crosswalks

- Meaning and use of stop and yield signs

- Dangers of parking lots

- Need for and use of safety belts

- Meaning of "pedestrian"

- Looking left and right before crossing the street

- Crossing streets at intersections rather than between parked cars.

6. Teaching methods/materials used:

- Film

- Safety song

- Children's creations

- Creating their own songs, art, work, poetry, stories, skits, films, videotapes

- Games such as a maze, dot-to-dot game, "What's Wrong With This Picture?" word and picture match-up, true-false quiz, poster to color, and picture depicting safe and unsafe pedestrian behaviors

- Memorization of rules

- Story

- Use of parents, grandparents, and young aduits to teach children certain rules.

7. Measurement of children's knowledge and behavior:

- Measurement of children's knowledge through the quiz and entertaining games that children do themselves

- It is not known if there has been any attempt to measure children's behavior. 


\section{WASHINGTON}

Name of program: A. Teacher's Guide for the Primary Level Traffic Safety Education Kit, $K-3$ on "Pedestrian Safety"

1. Authors: Department of Public Instruction in Washington State.

2. Sponsors: Washington Traffic Safety Commission/NHTSA.

3. Date: 1976.

4. Age of children in the program: $K-3$.

5. Kind of safety taught: Pedestrian.

6. Unit reviewed: All units.

7. Format:

- This 68-page instructional kit is regarded as a working document with an evaluation space included in each lesson and blank spaces where teachers can submit their activities and jdeas. With the manual the teacher receives a $k \cdot i t$ of materials such as films, filmstrips, and Wait Disney:s Pedestrian Safety Set.

- "How To Use This Kit" (an overview of pedestrian areas taught, discussion of the importance of teacher feedback).

- "Pedestrian Safety Objective" (eight behavioral objectives, focus is on student's knowledge of traffic safety)!

- Lesson plans, each of which has the following:

-- Area (of education)

-- Topic (of lesson)

-- Page

-- Grade (primary)

-- Objective (behavioral)

-- General objectives (such as self-concept, decision-making, values clarification and social responsibility)

-- List of resources (materials)

-- Description of activities

-- Evaluation (rating on scale of 1-5 from "Ugh!" to "Wow!")

-- Suggested ways to incorporate the activity into other subject areas

-. Dittos; songs etc., if needed in the lesson.

- The few dittos included in the guide vary. A few have sketchy drawings of items. For example, one shows a tree, traffic light and stop sign drawn in no context--one in the center of the page, others in the corners. Another ditto shows a situation with a stranger trying to pick up children. The children depicted are wearing 
up-to-date clothes, and an attempt is made to depict minority persons. Another ditto provides ample details of the traffic environment (grass and pebbles and other details making the picture look more realistic), but the car and truck and people look as if they were from the 1950s.

8. Concepts taught in the following order:

- Existence of nonverbal signals

- Meaning of and appropriate pedestrian and driver response to traffic signal light colors and stop sign

- Meaning and use of signs and signais

- Traffic signal light review

- Scanning

- Review of traffic signal lights and signs

- Concept that narrow streets are easier to cross than wide streets

- Dangers of crossing the street at mid-block

- Procedures for finding a place on the street where there are no parked cars and crossing the street

- Crosswalks and intersections

- People concerned with safety, e.g., fireman, policeman

- Proper interpretation of policeman's hand signals

- Proper use of "Walk/Don't Walk" signals

- Review of traffic signal light colors

- Correct procedures for crossing at an intersection

- Safety rules for going to and from school

- Name, address and telephone ( 3 consecutive lessons)

- Community helpers

- What to do in an emergency or if you're lost

- Difference between strangers and friends, and appropriate procedures to take if approached by a stranger (several lessons) 
- Community helpers (several lessons)

- Nighttime visibility

- Dangers of walking and driving in bad weather

- Daily weather

- Other hazards to pedestrians as introduced by "Super sidney Takes a Safe Walk"

- Procedures for becoming a good traffic safety-consclous person (not defined).

9. Pedestrian instructions given:

- Stop when the red signal light faces you, wait on the yellow, and go on the green.

- Obey signs and traffic signals.

- Scan for cars.

- Do not cross the street between parked cars; find another place to cross.

- Cross the street at a place where there are no parked cars.

- Cross on the crosswalk.

- Cross at intersections.

- Obey the policeman.

- Obey the "Walk/Don't Walk" signal.

- Don't accept anything from strangers or talk to them.

- Go to a community helper in tíme of trouble.

- Be careful when walking outside during bad weather.

- Wear white when walking at night.

N.B. As no list of traffic rules is given and they are not interspersed throughout the lessons, the above list of traffic rules may not be complete.

10. Teaching methods/materials used:

- Filmstrips

- Wa1t Disney Pedestrian Safety Set 101-C 
- Discussion

- Poems

- Games such as "Red Light Green Light"

- Songs

- Visits to the classroom by policeman and others

- Dittos - following activity directions and coloring

- Dramatization of (pantomiming) a poem

- Role playing traffic situations in simulated traffic environment (in playground and classroom)

- Memorization (e.g., traffic safety vocabulary, name and address)

- Field trips to real traffic environment to observe signs, practice crossing a street

- Interpretation of pictures

- Fingerplay

- Developing a list of safety rules

- Bulletin board (designed by teacher)

- Writing (e.g., name and address)

- Placing identification badges on children's mailboxes

- Flash cards

- Drawing pictures

- Receiving "supersaferoo certificate" (signifying child's knowledge of safety procedures)

- Orawing pictures

- Role play with puppets

- Problem solving cards

- Telling stories

- Listening to story

- Writing letter to community helper 
- Experiment looking at visibility of different colors in a darkened room

- Experiment seeing if water, ofl and other things make a surface slippery

- Calendar of weather

- Counting number of days with different types of weather

- Role playing being "Super Sidney" and "Boomer," two filmstrip characters

- Washington State Teacher's Guide for Traffic Safety (to be used as a resource).

11. Measurement of children's knowledge and behavior:

- None suggested other than direct observation of children as they carry out the activities.

12. Evaluation of this traffic safety curriculum:

- Feedback from teachers who have used the curriculum. 
WASH INGTON

Name of program: Elementary Traffic Safety Education: A Resource Guide for Teachers $(K-6)$

1. Authors: Washington State Department of Public Instruction.

2. Date: 1974.

3. Age of children in the program: $K-6$.

4. Kind of safety taught:

- Pedestrian

- Bicycle

- School bus

- Auto passenger.

5. Units most closely reviewed: Pedestrian safety.

6. Overall format:

- Foreword (need for traffic safety education)

- Acknowledgements

- Introduction (This section states limitations of children's capabilities in traffic situations--a very general statement that elementary children are not miniature adults. The introduction also explains how the curriculum is organized.)

- There is no breakdown of activities for children in different grades. Traffic safety content is divided into two sections, however, $K-3$ and 4-6.

- Each lesson in each unit suggests behavioral objectives and several activities from which the teacher can choose for teaching children in his or her respective class.

- "Suggested Behavioral Objectives" (K-6)

- "Traffic Safety" (general suggestions for teaching pedestrian safety)

- "Content" (concepts to teach children) is listed in the left-hand side of the page while "Learning Experiences" (learning activities) are put on the right

- "Washington State Motor Vehicle Laws--Pedestrians' Rights and Duties" 
- The last sections of the guide include the following sections: song and poems; traffic safety audio-visual materials available from the intermediate school districts; additional films, AAA pamphlets; Evergreen Safety council pamphlets; U.S. Department of Transportation pamphlets; and Washington State Patrol materials. Lists of films, one- or two-page brochures, book lets with modern traffic signs are included in these sections. One of these pamphlets explains why traffic safety education is needed by children. It lists four general reasons for children's traffic accidents (jaywalking, walking on the wrong side of the road; walking in the dark without light clothes, waiting in the street). It also lists safe pedestrian practices such as watting on the curb for a bus.

7. Concepts taught in this order:

- Name and address

- Communtty helpers

- Safest route to and from school

- Go directly to school without tarrying

- Cooperate with safety patrol

- Beware of strangers

- Traffic light colorij - how to read and obey them/spatial placement of traffic light coiors

- Traffic signs--shapes and meaning

- How to cross at intersections (different kinds of intersections, directional signals on cars, cross streets only on corners, don't go between parked cars)

- Blind spots where pedestrians can't be seen by drivers--driveways, parking lots

- Sidewalk behaviors; where to walk on sidewalks (right side)

- Where to walk when there is no sidewalk

- Meaning of "pedestrian."

8. Teaching methods/materials used:

- Memorization

- Parent involvement in planning route

- AAA unit on Safest Route to school 
- Walks on real streets near school

- Maps

- Pictures

- Stories

- Flash cards

- Safety patrol visit to classroom

- Role-play

- Orawings of traffic lights from memory

- Models

- Puzzles of signs

- Safety songs

- Films

- Experiments - trying to run fast and stop (1ike cars).

9. Measurement of children's knowledge and behavior:

- Observations of children's behavior when they're walking on real streets

- Observations of how children perform activities. 


\section{WASHINGTON}

Name of program: Harvey the Talking Motorcycle

1. Source of information about the program: Lyle E. Smith. Traffic Safety, Apri1 1972.

2. Sponsor of program: Tacoma Police Department in Tacoma, Washington.

3. Date: 1971-1972.

4. Age of children in the program: four to eleven years.

5. Program description:

- A police officer and "Harvey," a motorcycle with a tape hidden inside, came to schools to talk to young children about traffic safety. The policeman and Harvey carried on a conversation about the importance of pedestrian and bicycle conduct, the role of the police and the place of motorcycles and equipment in police work.

- Next there was shown an eight-minute cartoon which focuses on either pedestrian or bicycle safety and reviews safety rules.

- Afterwards, there was a question-answer period.

6. Concepts taught:

- Unknown

7. Teaching methods/materials used:

- Film with cartoons

- Discussion

- Visit by a police officer

- A "talking" motorcycle. 


\section{WISCONS IN}

Name of program: Project Impact

1. Program products:

- It's Your Move, a series of nine 15-minute programs shown on Wisconsin's educational T.V. network.

- Teachers' guides, one for K-6 and one for junior high.

2. Sponsors: Governor's Office of Highway Safety in the State of Wisconsin in cooperation with the Wisconsin Department of Public Instruction.

3. Date: 1974-1977.

4. Age of children in target audience: K-Junior High.

5. Kinds of safety taught for $K-3$ :

- Pedestrian

- Bicycle.

6. Programs/materials reviewed: Teacher's Guide.

7. Format:

- The T.V. program uses a mini-drama format showing $K-3$ chiidren coping with a real traffic situation safely.

- The two programs on pedestrian practices for the $K-3$ age group are:

-- "Crossroads" (teaches the basic elements of traffic and basic pedestrian skills)

-- "The Safest Way" (teaches children to be conscious of safety and to select appropriate and safe methods of transportation).

- Each lesson in the Teacher's Guide contains the following information:

-- Program theme

-- Program objectives (approximately four per lesson)

-- Synopsis of the program

- Vocabulary children should learn

-- Suggested activities (usually three or four).

Each lessons covers two pages. The entire booklet is 19 pages long, covering nine programs.

- Wisconsin residents may videotape the programs off the air and use the series as long as it appears on the Wisconsin Educational T.V. Network. The series is run each Fall and Spring. Videotapes can also be purchased for a small fee from the ECB Videotaping Service in Madison, Wisconsin. 
8. Concepts taught:

- That certain kinds of behavior are appropriate/inappropriate for the sidewa lk

- How to cross at an intersection with a traffic light signal

- The pedestrian's need to see, and obstacles impairing the pedestrian's vision.

- The pedestrian!s need to listen for traffic and ability to discriminate among different types of vehicles

- Dangers to pedestrians on sidewalks

- Meaning of "vehicle," "curb," "pedestrian," "signal," "traffic," and "controlled intersection"

- Use and meaning of road signs

- That choosing the safest means of transportation depends on the route to the destination and the conditions under which one is traveling

- Crossing streets at the corner

- The dangers of walking between parked cars

- The dangers of walking on railroad track;

- Meaning of "raflroad crossing" and "short cut"

- Need for and use of sounds such as horns, sirens, bells.

9. Teaching methods/materials used:

- Television dramas

- Making model of traffic light signal

- Flash cards with pictures of signs (made by teacher)

- Signs made by children

- Discussion

- Design and use of safety stationary

- Mapping children's safest route to school

- Using existing maps of the neighborhood.

10. Measurement of children's knowledge and behavior:

- No instruments are provided or suggested. 


\section{WISCONSIN}

Name of program: My Safety Coloring Book and My Safety Coloring Book Teacher's Guide

1. Sponsors: State of Wisconsin Governor's Office of Highway Safety.

2. Date: Unknown

3. Age of children for whom materials are intended:

- Preschool

- $\mathrm{K}-3$.

4. Kind of safety taught:

- School bus

- Pedestrian

- Bicycle

- Car passenger.

5. Materials reviewed: Both the coloring book and Teacher's Guide.

6. Format:

- The Teacher's Guide, an eight-page booklet, is devised to complement each page of the children's coloring book. The format in the Teacher's Guide is as follows for each lesson:

-- Concept taught

- Discussion questions and answers

- Activities.

At the end of the booklet is a list of other materials available from the Office of Highway Safety in Madison, Wisconsin.

- The children's coloring book is a 16-page book let with one

illustration per page and one short phrase teaching a traffic rule on the top of each page. The common character portrayed on every page is Spot, a dog wearing a school patrol safety belt; the dog sits on the bus, acts like the school patrol, and looks as if he were giving advice. The other characters in the booklet, children primarily, are representative of different races and are engaged in different

traffic-related activities such as fixing a bike, crossing the street and boarding. a school bus. The apparel worn by female figures is always a skirt; no attempt has been made to portray girls as wearing a variety of clothes. Only boys are shown riding bicycles; girls are sitting or walking, thus showing females as being less active--more passive. The one girl standing near a bicycle is watching an older 
male--perhaps father or brother--fix the bike. Police in the illustrations are always male and always fat. The traffic environment is illustrated with a considerable amount of detail or contextual clues to aid children in understanding what the pictures represent. Signs, for instance, have posts and are clearly stuck in the ground. Pebbles and grass show where sidewalks cease and grass begins. Many of the scenes are rural; a few appear to be suburban.

7. Concepts taught in the following order:

- Safe walking rules

- Where to walk when there's no. sidewalk

- Approprlate behavior at crosswalks

- To obey traffic lights.

8. Pedestrian instructions given:

- Walk on the sidewalk when one exists.

- Walk on the left shoulder of the road facing oncoming traffic.

- Walk between the lines of the crosswalk.

- Obey traffic ilghts.

- Cross streets at the crosswalk.

- Wear bright clothes in daylight; reflective material at night.

- Don't cross midblock or at an angle.

- Look a11 ways at corners for cars, buses, trucks.

- Stop at the curb to check for traffic before stepping into the street.

- Cross the street on the green traffic light.

- Obey the "Walk/Don't Walk" signal.

- On the "Don't Walk" signal, finish crossing the street if you already started or stay on the curb if you haven't begun to cross.

N.B. Most of the above rules are provided in the Teacher's Guide. It appears as if one of the teacher's most important tasks involves helping children to interpret the coloring book fllustrations, as few words are used in the coloring book and the safety rules pointed out are not necessarily clear. For example, under the caption "Safe Walking Rules" you see a little girl holding a man's hand while walking on the sidewalk and you see her waving to the policeman and 
dog across the street. A young child might focus on her action of waving or on the police or the dog rather than the fact that all pedestrians are walking or standing on sidewalks.

9. Teaching methods/materials used:

- Coloring pictures in the coloring book

- Discussion

- Making safety posters depicting rules

- Using Wisconsin's "Mini-Intersection Kit," a canvas mat simulating a real traffic intersection and role playing appropriate pedestrian behavior

- Teacher demonstrating appropriate pedestrian behavior in real traffic environment

- Teacher instructing children in safe pedestrian behavior in real traffic environment and children practicing appropriate behaviors

- Observation of safe/unsafe pedestrian behavior and traffic environment in real traffic environment

- Illustrating concept on the chalkboard

- Making model of traffic signal light.

10. Measurement of children's knowledge and behavior:

- No instruments or other form of measurement provided or suggested. 


\section{WISCONSIN}

Name of program: Project Traffic (Traffic Related Activities for Future Involved (itizens)

1. Developers: Robert Wing, School Safety Coordinator for Madison Public Schools and several K-8 teachers.

2. Date: 1974-1977.

3. Age of children in the program: $K-8$.

4. Kind of safety taught:

- Pedestrian

- Bicycle

- Passenger

- School Bus.

5. Units reviewed: None.

6. Format:

- The K-2 portion of the program consists of an activity card file with a supplementary material package. Each activity card contains the following:

-- Recommended grade level (K-2 Activity Cards apply equally to all K-2 students; they are not further broken down by grade).

-- Related academic area

-- Learning activity directions

-- Materials needed

-- Indication of child or teacher-directed activity (i.e., whether done by individual child or by the class as a group led by the teacher).

-- Need for supplementary materials (i.e., dittos, transparency masters, puzzles, games, tests, and teacher information sheets).

N.B. No lesson objectives or background information are provided. Some lessons refer to transparancies we did not receive.

7. Concepts taught in the following order:

- Traffic light, "Walk/Don't Walk" light

- Safety rules, traffic signs

- Pedestrian's need for visibility, problems of clothing blocking visibility 
- Safety rules

- Dangers of driveways, where to walk when there's no sidewalk, how to cross the street safely

- How to cross the street safely, improper street crossing behavior

- Meaning and appearance of traffic signs

- Safety concepts as introduced through safety riddles (no concepts are sugges ted)

- Safety rules

- Traffic signs, focus on stop sign

- Role of safety patrol, proper way to cross the street

- Traffic signs and other safety features as introduced in the filmstrip "Signs of Life"

- Careers

- The roles of police, crossing guards, school bus driver, and parents as related to safety

- Safety principles and situations

- Helping Hand Parents--who they are and what they do

- Personal information (name, address, phone number); role of the police officer

- Most direct route between home and school, dangers of strange animals, no trespassing concept

- How to deal with strange animals

- Traffic noises--what they mean, responsibility toward helping handicapped children in traffic

- Role of policeman, what to do when lost

- Use of curb, proper curb safety habits

- Meaning of traffic light colors

- Problems with nighttime visibility

- How to cross the street where there's a traffic light

- Waiting on the corner to cross the street 
- Oifficulties of maintaining control over the body when moving rapidly

- Meaning of words "Walk," "Don't Walk," "Stop," "Go," and "Wait" and their relationship to traffic signals

- Safety concepts presented through poems

- Correct usage of safety colors

- Meaning of red and green colors with regard to safety

- Safety at corners

- How to judge distance

- The safest way to school

- Traffic safety helpers

- Basic shapes

- How to be safe during bad weather

- Emergency procedures during a tornado or storm

- Source of $11 \mathrm{ght}$

- Importance of wearing reflective tape in the dark, 1.e., at night

- Safety concepts taught through stories

- Safety features in a town

- Pedestrian rules

- Dangers of jaywalking, safest route, where to walk when there are no sidewalks, unloading from buses and cars, pedestrians's right of way with bicyclists.

- Traffic signs

- Meaning of different kinds of pavement markings

- Reason for traffic signs, policemen, vehicle turn signals, traffic rules, bicycle reflectors, seat belts

- Safest route to school

- Responsible ways to help a sick child walking home from school

- Pedestrian safety practices (not defined)

- Meanings of shape and color of traffic signs 
- Problems involved with pedestrian safety

- Sign shapes

- Special street signs indicating presence of handicapped children

- Sign shapes

- Safe ways to walk to school

- Safe places to cross the street

- How to cross at intersections

- Concepts taught in It's Your Move television series.

8. Pedestrian instructions given:

N.B. Traffic rules are not listed on the activity cards. A teacher would have to know the rules, consult other sources, or try to derive them from the activities/concepts listed above. Traffic rules, however, may be written on the dittos or supplementary materials that accompany the activity cards.

9. Teaching methods/materials used:

- Construction of models (of traffic light, signs, village, etc.)

- Representational activities such as designing safety pictures on bookmarks, making safety books, drawing pictures

- Demonstration by child (e.g., how umbrella blocks vision)

- Role play in simulated traffic environment (in classroom or outside)

- Pretending with finger puppets, puppets

- Dittos (coloring and following directions and interpreting pictures)

- Poems

- Riddles

- Diary recording traffic situations

- Visit to classroom by Helping Hand parent, safety patrol, police officer, and various kinds of drivers

- Demonstration of safe pedestrian practices by safety patrol

- Discussion

- Films, filmstrips, slides, It's Your Move television series 
- Telling stories

- Tape recording of traffic sounds

- Games such as "Red Light, Green Light."

- Fingerplays

- Reading stories

- Experiments estimating distance children move, seeing how reflective tape can be seen in the dark

- Questionnaire to be filled out by parents and children

- Using flannelboard to tell stories

- Visit to real traffic environment to observe safety features

- Mint-Intersection Kit (containing a $15^{\prime} \times 15^{\prime}$ canvas mat, mini-traffic signal, fifp chart, and teacher's guide, i.e., a simulated traffic environment.)

10. Measurement of children's knowledge and behavior:

- A "Pedestrian Pretest and Posttest" is listed in the K-2 activity cards table of contents but was not included with materials sent to us.

11. Evaluation of the effectiveness of this traffic safety curriculum:

- None was mentioned: 


\section{WYOMING}

Name of program: Wyoming Traffic Safety Education

1. Primary authors: Wyoming State Department of Education.

2. Sponsors: Wyoming Governor's Office of Highway Safety.

3. Date: 1979.

4. Age of children in the program: $k-6$.

5. Kind of safety taught:

- Pedestrian

- School Bus

- Bicycle

- Auto Passenger

- Skateboard

- Blizzard.

6. Unit reviewed: Pedestrian.

7. Format:

- The foreword explains to teachers how to use the guide, and lists a number of organizations from both the public and private sectors to which teachers can write for additional traffic safety education materials.

- The six safety units listed above are each color-coded a different color. Each unit is further divided into smaller units or lessons. Each lesson contains the following:

-- Behavioral objectives designed to reveal the extent of children's knowledge of traffic rules as well as the extent of their traffic safety skills.

-- A list of teaching activities sometimes described in a few sentences and sometimes given in detail. For example, one activity suggests constructing a model traffic light but gives no suggestions as to how to do it. other activities provide the words of poems, songs, and games.

-- To the left of each activity the designated grade levels for which the activity is appropriate are indicated. The majority of activities are designated for $K-6$; apparently, either developmental differences between groups of different ages are not perceived as great or the teacher is expected to adapt each activity to the abilities of his or her students. 
-- Sometimes there are sections providing background information for the teacher, e.g., citing traffic rules or explaining changes in traffic signs. However, there are no sections informing teachers about the kinds of accidents befalling $K-6$ children, about reasons for children's accidents or about why particular concepts were chosen for the curriculum.

-- Some dittos and games have been integrated into the lessons.

- At the end of each unit (such as the pedestrian unit) a bibliography of resources such as additional curriculum materials and manuals on traffic laws and safety devices produced by Wyoming or the Federal Government is included.

8. Concepts taught in the following order:

- Meaning of "pedestrian" and pedestrian safety rules observed in Wyoming. A game in this lesson introduces a multitude of concepts such as using the crosswalk, looking both ways before crossing the street, hitchhiking, yielding, but none of these concepts have been introduced previously, as this is the first lesson.

- Scanning for danger; I.D.P.E. concept (Identify, Predict, Decide and Execute); reasons that children are not seen by drivers; directions; the driver's difficulty in stopping immediately when a pedestrian darts. out in the street; idea that some cars like Bruce the Killer Car, may try to hit and kill child pedestrians.

- The shapes, colors and meanings of over 15 different signs; the meanings of the following pedestrian related words: "stop," "caution," "yield," "one way," "railroad," "no admittance," "danger," "walk," don't walk," "red," "yellow," "green"; 10 safety rules, such as wearing white when walking on the street at night, waiting at the curb, and looking for turning cars; careless pedestrian actions such as petting strange dogs, jaywalking and chasing a ball into the street; the meaning of traffic light signal colors and appropriate pedestrian behavior at traffic lights.

- The pedestrian's problems of visibility when walking on or near the street at night--the need for reflective tape or white clothing.

- Poor visibility caused by bad weather conditions, poor light and other conditions such as dirty windshields and clothing which impairs pedestrian vision--how poor visibility may lead to traffic accidents.

- The dangers of strangers and what to do when approached by a stranger.

9. Pedestrian instructions given:

- Cross the street when the green traffic slignal light faces you, however, watch for turning vehicles. Don't cross on the red or yellow signals. 
- Vehicles should yield right of way to pedestrians who are crossing within crosswalks or within unmarked crosswalks at intersections.

- Walk on the right half of the crosswalk.

- Don't walk in the roadway when a sidewalk is provided; walk on the sidewalk.

- When there is no sidewalk, walk on the left side or shoulder of the road facing trafic.

- Don't hitchhike.

- Obey traffic signs and traffic signals such as "Walk/Don't Walk."

- Use the crosswalk.

- Don't run in the street after a ball.

- Obey your safety patrol.

- Look left, then right, before crossing the street.

- Don't play in the street.

- Stop, look and listen before crossing the street.

- Obey traffic signs.

- Don't play behind cars.

- Use your eyes to spot cars while you're crossing the street, waiting for a ride, or walking on the sidewalk.

- Wear or carry something white or carry a light when walking on the street at night.

- Keep your umbrella away from your face so you can see.

- Don't step into traffic lanes from behind or in front of parked cars.

- Cross intersections at right angles, not diagonally.

- Wait on the curb until you can cross safely.

- Look for traffic before entering or disembarking from a vehicle.

- Don't jaywalk.

- Don't pet strange animals. 
- Beware of cars moving in driveways when you cross the driveway path. - Don't accept gifts or rides from strangers.

10. Teaching methods/materials used:

- Discussion

- One pedestrian field trip (after all concepts are presented in the classroom) to emphasize concepts previousily taught

- Coloring a ditto illustration

- Games such as "Pedopoly," a board game which emphasizes safety doncepts; an obstacle course; matching shapes; connecting dots; a magic number game involving substituting letters for numerals in division, addition; subtraction and multiplication problems to figure out traffic safety terms

- Measuring student height to that of cars, bushes and fences

- Writing short poems or stories on traffic safety concepts

- Experiment seeing how fast chlldren who are running can come to a complete stop

- Role-play or puppet show to enact a play about Bruce the Killer Car who tries to hit children and kill them

-. Finger plays

- Songs

- Maze

- Bulletin board of slogans for pedestrian safety

- Memorization of rules

- Interpreting diagram of an intersection on a chalkboard

- Model of a traffic light

- True-false quiz

- Peg board to construct sign shapes and traffic safety words

- Experiment darkening room to determine visibility of light and dark colors

- Telling stories. 
11. Measurement of children's knowledge and behavior:

- At the end of the unit there is a "Pedestrian Rating Scale," which consists of 15 statements about correct pedestrian behaviors.

Student rate whether they always, usually or rarely practice safety rules such as the following: cross streets only at intersections or crosswalks; stop, look both ways, and look for turning cars before crossing the street. A few rules in this rating scale have rarely or never appeared in the curriculum activities-using street routes rather than alleys or shortcuts, obeying safety patrols and using a safe route going to any destination. Students are to use this rating scale on themselves, discuss it with the class and with their parents. 


\section{PEDESTRIAN PROGRAMS FOR PRESCHOOL CHILDREN \\ Sponsored by \\ THE PRIVATE SECTOR.}


Name of program: Preschool Children in Traffic

1. Authors: Sylvia Ross, Department of Traffic Engineering and Safety, American Automobile Association (AAA).

2. Sponsors: AAA.

3. Date: 1976 .

4. Age of children in the program: two to six-year-olds.

5. Overall program:

- A book for parents - Preschool Children in Traffic: Parents' Guide For Action

- Four books for children - When I Go outside for ages two-and-a-haif to six; I Listen and Look For Cars Coming for ages three to six; How I Cross a Street for ages four to six; Traffic Signal Lights for ages five to six.

6. Books reviewed: All.

7. Format of Preschool Children in Traffic: Parents' Guide for Action:

- The cover is a picture of preschool children of different ethnic and racial backgrounds with a traffic light in the background. Pen and ink drawing with black, grey, and rust shading are used.

- "Reducing young children's pedestrian accidents" discusses the purpose of the program and gives guidelines for using the book series with children.

- The next section describes the titles of children's books, ages of children for which they are intended and pedestrian safety concepts introduced in each book.

- "Young pedestrians and traffic accidents" gives statistics for children's pedestrian accidents and describes typical accidents.

- "Developmental characteristics that influence a child's behavior" discusses physical, mental, emotional and social characteristics that affect children's behavior in traffic. 
- "What actions can parents. take?" This six-page section describes general actions that parents can take to supervise and teach their children in traffic situations.

8. Format of children's books:

- The covers show quite realistic drawings of preschool children of different ages. A toddler with definite toddler characteristics (height, interest in pull-toys) is on the cover of the first book. Slightly older children are depicted on the covers of booklets for children three to six and four to six years of age. A five or six-year-old is on the last book which is intended for five and six-year-olds. Two children with minority characteristics - one with glasses and one who is a Black American - are shown. Wearing apparel is up-to-date and attractive.

- "To Parents" is a section directed at parents, reminding them of the Parents Guide To:Action and giving them practical tips about ways to reinforce concepts presented in the booklet.

- The children's section of each booklet is about 11 or 12 pages long. At the most there are four short: sentences per page. Of ten there are one or two sentences on the page. Illustrations play a very important part of the children.'s booklets; the illustrations, in most cases, fllustrate the ideas being: stated in the text. In a few places, such as the driveway. scene, the illustrations go beyond the text in. telling the story. In the case just mentioned, the illustration shows car exhaust and light rays from rear view lights on a car backing out of a driveway, but the text just mentions that cars in driveways of ten back up. There is usually one illustration per page or one illustration on a two-page spread. The children and adults depicted in each booklet remain constant throughout the. book. For example, the toddler seen on the cover of When I Go Outside is the same child all through that booklet.

- Suburban traffic environments are depicted.

9. Order of concepts taught in When I Go Outside (for two-and-a-half to six-year-olds):

- Safe and unsafe play areas - yard and street respectively

- Use of streets - vehicles such as cars

- Words and concepts for people who walk, ride, and drive 
- Word for and use of "sidewalks"

- Word for and meaning of "curb"

- How to cross street mid-block with an adult - breakdown of skills/actions needed (stop, stand, look)

- How to judge whether a car is approaching or driving away

- Driveways as hazardous - as having cars which back up

- How to tell if a car is backing up

- What to do when a car is backing up in a driveway.

10. Pedestrian instructions given (in all four children's booklets):

- Play in the yard, not in or near the street.

- Only go near the street if an adult is with you.

- Walk on the sidewalk or on the grass, not in the street.

- When there are no sidewalks, walk next to the street. (The lllustration shows them walking on the left, facing oncoming traffic.)

- Stop at the edge of the street before you come to any street and look for cars, trucks or buses coming towards you.

- If you're on a driveway, look for cars that may be backing up. (The illustration shows exhaust and back-up lights and movement which are all cues for which the pedestrian should look.)

- Listen for cars and try to tell from the sound whether cars are coming or going.

- Use your eyes to look one way and then the other way for cars that are coming. (The illustration teaches them to look left then right.)

- If anything such as a parked car or bush keeps you from seeing cars that are coming, find another place to cross the street, a place where you can see a long way in both directions and a place where drivers can see you too.

- When there are no cars coming, you can cross the street. 
- Don't run across the street to meet someone; stay in your yard and let them come to you.

- If there are crosswalks on the street and you want to cross, walk inside the lines of the crosswalk.

- Stop when you come to a stop sign facing you and watch to see that cars stop when they come to stop signs.

- When cars come to yield signs, they should stop to let you cross, but wait and watch to make sure the driver sees you and stops.

- Wait until the driver waves you to cross before you walk in front of a stopped car.

- Look for cars turning onto the street you want to cross before you cross. If a car is close to the corner, wait for it to turn and pass.

- Look for traffic signal lights at corners and above the street.

- Watch the traffic light straight across the street you are going to cross. On the red light, don't cross; stay on the sidewalk back from the curb. Wait--you don't have time to cross on the yellow signal. On the green light, look to see if cars are coming before you cross.

- When the "Walk" sign flashes, you may cross the street, but first look for turning cars.

- When the "Don't Walk" signal flashes, wait on the sidewalk.

- At intersections with traffic lights, look to see if cars behind you or in front of you are turning on to the street you want to cross, and: if they are turning, wait for them to pass. Even if the "Walk" light flashes, wait until all the cars on the street you want to cross have stopped. Then walk quickly across. Even when those cars on the street you want to cross have stopped, look for turning cars.

11. Teaching methods/materials used:

- Learning experience in a real traffic situation with parent as teacher--after reading the book. Techniques used to teach the above concepts include: 
-- Walking with the child on the sidewalk and pointing out sidewalks and streets, curbs, yards etc. and telling the child where the walker's territory is

-- Stopping for a definite time before crossing every street

-- Squeezing the child's hand to signal stopping at the curb or edge of grass

-- Kneeling by the child's side and looking for vehicles so the child can model the adult's scanning behavior

-- Showing the child vehicles backing up - listening for noise, looking for lights and exhaust, seeing how cars look from the rear.

- Book which uses the following techniques:

-- Realistically drawn children, adults and traffic situations

-- Many illustrations depicted from child's height in order to depict the situation as a child might see it

-- One or few illustrations and words per page

-- Simple language

-- The same characters depicted throughout

-- A suburban-looking nelghborhood shown in a realistic manner

-- On the two pages showing a car coming closer and a car going away, however, a less realistic, more diagram type of drawing is used: the same car and driver outlines are superimposed on one another in an attempt to show movement.

12. Order of concepts taught in I Listen and Look for Cars Coming (for ages three to six years):

- To listen for cars coming and going

- To watch for cars coming and going

- To look to each side for cars coming down the street

- How to find a good place to cross the street mid-block when obstacles such as bushes obstruct the view of the street.

- The sequence to follow in crossing the street - stop on curb; listen for cars; look for cars on your side of the street (this isn't taught earlier); look for cars from the other side; cross if no cars are coming

- To stay in the yard and wait for visitors to come to the yard rather than darting across the street to see them. 
13. Teaching methods/materials used for teaching concepts in I Listen and Look:

- Learning experience in real traffic situations with parent as teacher. This experience comes after reading the book. Specific techniques include:

-- Modeling appropriate behaviors such as looking left and right, pointing at cars, etc., step by step

-- Asking the child to copy adult modeling behaviors

-- Using designated simple concepts and language such as "one way" and "the other way" rather than more difficult concepts and language such as "left" and "right"

-- Asking questions of the child to encourage him or her to look. listen and judge cars more carefully

-- Explaining why: such actions should be taken.

- Book which uses similar techniques described for the earlier book. When I Go Outside.

14. Order of concepts taught in How I Cross the Street (for ages four to six):

- People, cars and other moving objects move at different speeds

- Rationale for stopping when coming to a street

- Word and meaning of "traffic"

- Word and meaning of "corner"

- Word, meaning and use of "crosswalks"

- The existence of different kinds of corners with different safety features

- Use of stop signs by drivers and pedestrians

- Approprtate behavior when crossing with parent at a corner, wait until there's eye contact with the driver and look for turning cars

- Meaning of turn signals.

15. Teaching methods/materials used to teachiconcepts in How I Cross the Street:

- Learning experiences in real traffic situations with parent as teacher (after reading the book). Specific teaching. techniques used: 
-- The same as those mentioned earlier

-- Giving children an opportunity to observe real traffic situations from a higher position.

- Book which uses techniques similar to those in previous books.

16. Order of concepts taught in Traffic Signal Lights (for ages five to $s|x|$ :

- Use of traffic lights

- Where traffic lights are located

- Meanings of traffic light colors and appropriate pedestrian behaviors for each color

- Location and meaning of "Walk" "Don't Walk" signs

- Looking and waiting for all cars (including turning cars) to pass before crossing on walk sign

- Walk across the street.

17. Teaching methods/materials used in Traffic Signal Lights:

- Learning experiences in real traffic situations with parent as teacher (after reading the book)

- Book which uses techniques already described plus:

-- One illustration which has three pictures showing a three part sequence

-- One lllustration showing a traffic situation from above.

18. Measurement of children's knowledge and behavior:

- No instruments for assessing behavior or knowledge are suggested, although parents are urged to observe their children's behavior informally.

19. Evaluation of this program:

- Teacher and parent questionnaires were distributed in four geographic areas where the curriculum was used and teachers and parents were asked whether, based on their own observations, they thought children behaved better in traffic as a result of the series. In general, reactions 
were reported to be favorable. Respondents believed that the materials were appropriate for the needs of children and parents. They also believed that the program was more beneficial when parents attended an orientation session first. No objective data or knowledge or behavior were collected. 


\section{GREEN CROSS SAFETY CLUB}

Name of program: Green Cross Child Safety Club

1. Sponsored: National Safety Council.

2. Date: 1978 .

3. Age of children in the program: three to five years.

4. Kind of safety taught:

- Home

- Pedestrian

- Car Passenger.

5. Program component reviewed:

- My Safety Book (for three-year-olds)

- My Safety Book II (for three-and-a-half-year olds)

- My Safety Book III (for four-year-olds)

- My Safety Book IV (for five-year-olds).

6. Overall format:

- Intended to be a series of five books which are sent to children at six-month intervals. Each book suggests activities parents can do with children and has a text and accompanying illustrations for children. The series focuses on many safety topics in addition to traffic safety, e.g., on safety measures to observe in the home and in an automobile.

- Format of My Safety Book:

-- Cover - a drawing of a black girl and white boy--both preschool age--holding the green cross symbol. A dog, cat and parrot are also in the drawing. Many colors are used--green, blue, red, purple, black, white, and yellow. 
-- "To The Parent" gives a general reason for children's need for parental supervision and safety instruction, mentions the kinds of safety focused on in this book and suggests general techniques to use in teaching the child safety habits.

-- Pop-out Picture-- seven colorful pictures that can be popped out and pasted in blank spaces in illustrations in the child!'s text.

-- "Now You Are Three", the child's text, consists of 13 pages and 4 basic sections

-- An introduction which recognizes some new skills three-year-olds have mastered and suggests that there are things children can do to avoid getting hurt

-- Three two-page spreads on home safety

-- Two two-page spreads on pedestrian safety

-- One two-page spread on passenger safety in cars

Formats of the other three booklets reviewed follow the same general format of the first booklet.

- The "To The Parent" sections of the remaining three booklets present the following information about the preschool child's developmental abilities:

-- The three-and-a-half-year-old's book let describes these limitations of that age child in traffic: farsightedness; short stature; lack of physical strength to run quickly; and emotional and impulsive nature.

-- The four-year-old's book let stresses the child's independence, imagination and initiative.

-- The four-and-a-half-year old's book let emphasizes the child's curiosity, as well as his/her need for self-esteem and exemplary adult models.

Each booklet usually contains two large illustrations depicting pedestrians observing model behavior in a traffic environment and/or parts of the traffic environment. Sometimes, the second of two illustrations consists of a picture puzzle into which the child inserts a pop-up picture. Illustrations in all booklets depict children and parents of different racial and ethnic groups in a middle-class suburban setting. The illustrations are very colorful, cheerful,: and pretty. The environment is depicted with realism in that objects represented bear considerable resemblance to the real objects. Most 
two-page spreads have two texts - large print to be read to children and small print for parents. The children's text includes many sentences and several different concepts per page. The parent's text describes learning activities that parents can do with children to reinforce the learnings presented in the child's text.

7. Concepts taught in the pedestrian safety section of My Safety Book I:

- Word, meaning and use of "sidewalks"

- Word and meaning of "walker"

- Word, meaning and use of "streets"

- Safe play areas - yard and playground.

8. Concepts taught in the pedestrian safety section of My Safety Book II:

- Meaning and use of "sidewalk"

- Meaning of "walker"

- Meaning and use of "yard"

- Meaning and use of "streets"

- Meaning and use of "driveway"

- Directions cars move in driveways

- Appropriate pedestrian behavior in driveways

- Need for walkers to look and listen to cars

- Ways to tell the proximity of a moving car from its sounds

- Ways to tell the proximity of a moving car from its appearance (size)

- Where walkers need to be in order to observe cars ("close to the street").

9. Concepts taught in the pedestrian safety section of My Safety Book II I:

- Need for walkers to listen and look for cars

- Ways to tell the proximity of a moving car from its sounds

- Ways to tell the proximity of a moving car from its appearance (size) 
- Where to cross the street (corner or crosswalk)

- What to do when vision is obstructed when scanning for cars

- When it's safe to cross the street ("when no cars are coming")

- How to cross the street (walk quickly from the edge on one side of the street to the edge on the other sidel.

10. Concepts taught in the pedestrian safety section of My Safety Book IV:

- Review of concepts taught in My Safety Book III, 1.e., procedures for crossing the street

- Definition, appearance, and use of "curb"

- Definition, appearance, and use of "corners"

- Definttion, appearance, and use of "crosswalks"

- Meaning, appearance, and location of stop sign for driver and pedestrian

- Meaning, appearance, and location of yield sign for pedestrian and driver.

11. Pedestrian instructions given in a 11 booklets reviewed:

- Walk on the sidewalk.

- Play in your yard or on the playground:

- If you are standing on a driveway, look for cars and get out of the driveway fast if you see a car coming.

- Listen and look for cars when you are a walker.

- Cross the street at the corner or crosswalk.

- When you want to cross the street, walk to the side of the street, look and listen to the left for cars and look and listen to the right for cars. When no cars are coming, step of $f$ the side of the street, walk across the street. quickly, and step up on the edge on the opposite side of the street.

- Stop when you come to a stop sign on the corner. 
- Be sure the car has stopped and the driver sees you and tells you to cross before you cross in front of a stopped car.

12. Teaching methods/materials used:

- Illustrations of model behavior and traffic environments

- Pasting activity with pop-up pictures described above

- Discussion about the book

- Learning activity on a real street with parent as teacher. This activity is done after reading the book in order to reinforce concepts presented in the boak

- Repetition of the pasting and reading activity described above.

13. Measurement of children's knowledge and behavior:

- No instruments for assessing children's knowledge or behavior are suggested.

14. Cost of set of materials to family: $\$ 15$. 
CHANNING L. BETE CO. TRAFFIC SAFETY EDUCATION MATERIALS

Name of program: Channing L. Bete Co., Inc.'s traffic education series for parents and children

1. Sponsor: Channing L. Bete, Co., Inc, in Greenfield, Massachusetts.

2. Date: 1979.

3. Age of children in the program: Not specified.

4. Names of books in series:

- How To Walk To School Safely

- What Parents Should Know About Children and Traffic

- Join The School Bus Safety Team:

5. Books reviewed:

- How To Walk To School Safely

- What Parents Should Know About Children and Traffic

6. Format of What Parents Should Know About Children and Traffic

(a) book for parents):

- This is a 15-page booklet with numerous cartoon-like illustrations of white middle-class parents and children in a suburban location. Women in the inlustrations are always wearing dresses and heels. There are very sketchy drawings of the traffic environment. The booklet is divided into several sections which are described below.

- The introduction explains to parents the importance of their roles as teachers of traffic safety. The introduction also encourages parents to take an active role in bringing about and/or supporting traffic safety education for children in the community and reminds parents who drive to be careful of children who are in or near the street.

- The next section lists six reasons that children are vulnerable in traffic situations. Children are said to: 
-- Play around traffic

-- Dash into the street to chase a toy or friend

-- Run across the street

-- Misjudge distances

-- Assume crosswalks are safe

-- Drive bikes into intersections.

- There is a list of five general methods parents can use to teach their children traffic safety.

- There is a section on teaching children appropriate pedestrian behaviors.

- There is a section on teaching children proper bicycling behaviors.

- There is a section on teaching children appropriate car passenger behaviors.

- There is a section listing precautions parents should observe while driving in order to minimize danger to children.

- "Give a Safety Lesson Today" lists about seven things parents can teach children when they take a safety walk around the neighborhood.

- There is a reminder that teaching children traffic safety and being a good example is an adult responsibility.

7. Format of How To Walk To School Safely (a book for children):

- This short booklet has the same style of illustrations mentioned regarding the other Bete booklet. (Of ten there are as many as five cartoons per page. The total effect is busy and confusing.)

- Sections focus on the safest route to school, safety features such as signs, traffic lights and signals and rules for crossing the road. At the end of the booklet, there's a true-false quiz which children can give themselves to evaluate how much they know about traffic safety.

8. Concepts taught in both books:

- Safest route to school--avoid busy streets

- Leaving early for school (time) 
- Don't play on the way to school

- Wait for a walk signal from the police

- Look left, right, left before crossing a street

- Walk don't run

- Meaning of signs

- Meaning of traffic lights and signals

- Play in safe areas

- Watch out for cars backing up in driveways

- Watch out for turning cars when crossing at any intersection.

9. Teaching methods/materials used:

- Verbal repetition of the dangers of traffic and safety rules

- Experience on real sidewalks and roads with parents insuring that children adhere to good safety practices

- Verbal explanations of why safety rules must be followed

- Parents modeling good safety practices

- Experience in real traffic environments with parents acting as teachers and pointing out traffic safety features such as crosswalks and traffic lights.

10. Measurement of children's knowledge and behavior:

- Children can use the true-false quiz to evaluate their knowledge of traffic safety. No other instruments are suggested. 


\section{SAFETY TOWN}

Name of Program: Safety Town

1. Author: Dorothy Chlad.

2. Sponsor: National Safety Town Center.

3. Date: 1973.

4. Age of children in the program: four and five-year olds.

5. Kind of safety taught:

- Pedestrian

- School Bus

- Bicycle

- Home

- Playground

- Medicine

- Fire.

6. Overall program includes:

- Twenty-hour course (two hours per day for two weeks) for children with minimal parent participation. The course is given at a Safety Town, a child-size model of a town with small streets, buildings, sidewalks, signs, traffic light, cars, trees etc, where children are instructed in traffic safety. Another part of the course is taught in a regular classroom where activities such as films, discussions, songs and art work can be carried out. One half hour of the course is taught on a real street.

- Instruction on many different kinds of safety besides pedestrian safety (playground, household safety, for example)

- The Safety Town General Information Guide

- The Safety Town Parent Guide

- The Safety Town Curriculum Guide (for instructors who teach traffic safety to children at a Safety Town). 
7. Program aspects most closely reviewed:

- The Safety. Town General Information Guide, Parent Guide, and Curriculum Guide.

8. Format of the Safety Town General Information Guide:

- Cover - drawings of children in traffic

- Statement of purpose of the National Safety Town Center

- Table of contents

- An introduction (the effectiveness of Safety Town)

- Safety Town's purpose, objectives, goals

- Safety Town objectives for children are:

-- "Learn, apply, practice safety procedures through indirect involvement in supervised simulated, and "real life" situations

-- Participate in activities in an atmosphere of educational fun for developing their confidence, self-rellance, and decision-making abilities

-- Develop a positive safety attitude and safety habits during the formative years

-- Acquire a general safety awareness upon which parents and schools can build

-- Develop the seed of responsibility for caring for themselves and others

-- Become acquainted with uniformed personnel and learn basic respect for law and order

-- Meet, learn and play with other children the same age."

- Safety Town objectives for parents are:

-- "attend daily classes with their children so they can properly continue safety education at home

-- better understand their children's physical and mental development as related to safety capabilities

-- practice safety procedures with their children by showing them the safest way to school, etc.

-- Keep abreast of safety laws and developments."

- The purpose and objectives of the National Safety Town Center

- A history of Safety Town - its effectiveness in various communities. 
9. Format of the Safety Town Parent Guide:

- An introduction (reason children need safety education, the importance of developing safety attitudes)

- Statement of purpose (encourage parent participation in children's Safety Town classes for 15 minutes every day. Parents observe the method of instruction.

- Schedule of Safety Town classes for children

- "Your Child's Safety Town Message to You" (several pages of safety rules written in letter format (Dear Mom and Dad...) as if a child were speaking ("First I stop my feet at the curb, EVERY time, then look left, right, and over my shoulder for turning cars and listen for sirens.")

- "Do You Really Understand Your Child?" (general tips to parents about how to interact with children-common-sense information)

- "How's Your Attitude?" (questions to parents about their own traffic records and pedestrian. behavior)

- "Philosophy of Safety Education"

- Purpose, objectives and goals of Safety Town.

10. Format of the Safety Town Curriculum Guide

- "Helpful Hints" (general teaching techniques to use with children, techniques most nursery school teachers would know)

- A daily curriculum guide for the two-week course. The guide gives a lot of logistics--when to show a film, which song to sing next, which safety feature to focus on next.

- Description of materials referred to in the activities-flannel board, treasure chest game, dittos, etc.

- Two resource books - ABC's of Child Safety, by Channing L. Bete Company, and Kids Will Be Kids, by the National Safety Council. 
11. Concepts taught which might be considered by Safety Town to relate to traffic safety:

- Name, address and te lephone number

- Following instructions

- Identifying houses and surroundings - sidewalks, crosswalks, corners, streets, curbs, buildings, traffic lights, signs

- Proper way to cross the street

- Importance of crosswalks

- Reason for stopping at curb

- Crossing with traffic light and walk light

- Meaning of "intersection," "pedestrian" and "motorist"

- Dangers of walking between parked cars

- Walking where there are no sidewalks

- Conditions when it rains or snows

- Walking on right of sidewalk, crosswalk, halls

- Dangers of playing outside after dark

- Proper procedure when crossing railroad tracks

- Dangers of trains

- Colors, shapes, and meanings of safety signs

- Dangers of chasing a ball into the street

- Safe places to play

- Proper way to enter and exit: from a car on the curb side

- Who strangers are and what to do if approached.

- What to do if lost

- Uniformed personnel, policeman, fireman, bus driver, patrol guard 
- How uniformed peronnel help people

- Being courteous and obeying rules

- Importance of thinking safety.

12. Pedestrian instructions given:

- Walk on the sidewalk.

- Walk on the right side of the sidewalk.

- Cross the street in crosswalks.

- Walk across the street - don't run. Never stop in the middle of the street.

- When crossing the street, stop at the curb, look left and right and over your shoulder for turning cars and listen.

- In the crosswalk, walk between the lines and on the right.

- When there is a traffic light, wait for the new green light, look left, right and over your shoulder to make sure no cars are coming, and then go. Do not cross on the red. Wait and get ready to stop on yellow.

- Don't play or walk between parked cars.

- Don't play near raflroad tracks.

- Stop when you see the flasher lights at the railroad crossing.

- Don't chase the ball when it rolls in the street. Wait until the ball stops rolling. (No further instructions are given.)

- Stay on the sidewalk and don't step into the street when you hear or see an ambulance.

- Listen for sirens.

- Don't chase ambulances, police cars or fire trucks.

- Obey safety patrol guards and police.

- When no sidewalks are available, walk on the left side of the street facing traffic. When a car comes, walk up onto the grass to get out of the car's way. 
- Don't play in the street: play in your yard, a friend's yard, or at the playground.

- When you are walking and come to a driveway, stop to look for cars backing out of the driveway

- Learn about and obey traffic signs and signals.

- Don't go with strangers - stay with friends, not alone.

13. Teaching methods/materials used:

- Film

- Songs

- Dittos

- Children's artwork

- Behavioral instruction in a simulated traffic environment (Safety Town) (3-1/4 hours)

- Discussion and explanation of rules

- Visit from policeman

- Posters

- Teacher modeling correct behavior in simulated traffic environment

- Group reinforcement (children cross the street together)

- Games

- Flannel board activities

- Stories

- One-half-hour training: instruction on real street.

14. Measurement of children's knowledge and behavior:

- An "Evaluation Report" is filled out on each child at the end of the two-week training period by the child's

instructor. The evaluation is basically an assessment of 
the child's knowledge of safety rules. The instructor notes whether the child made a "good response" or whether the child "needs practice" on 19 items, 7 items of which are directly related to pedestrian safety. The parent receives a copy of his or her child's evaluation report.

- On graduation day children demonstrate what they've learned in groups of children.

15. Evaluation of the Safety Town programs:

- Parent testimonials

- Parent questionnaires

- No formal summative evaluation. 
Name of program: Officer Friendly Resource Library

1. Name of book lets included in Resource Library:

- Handbook for Coordinating the officer Friendly Program

- Guide for Officer Friendly Selection

- Using the Officer Friendly Training Resources K-3

- Learning About Schools.

- Learning About Children K-3

- Planning Your Visits

- Getting Messages Back and Forth

- The Idea Book for Planning Your Visits.

2. Compiled by: Marjory L. Coffing and Norman M. Singer at the Center for Vocational Education, 1960 Kenny Road, Columbus, Ohio 43210.

3. Sponsored by: The Sears-Roebuck Foundation.

4. Date: 1975 .

5. Age of children in the program: $K-3$.

6. Kind of safety taught:

- Pedestrian

- Bicycle

- Bus

- Automobile

- Household

- Neighborhood 
- School

- Seasonal

- $\quad$ Drug

- Fire.

7. Unit reviewed: All, particularly the sections relating to pedestrian safety.

8. Format:

- The booklets listed above present the purpose of the officer friendly Program and give guidelines for operating and teaching the program.

In general, the officer Friendly Program is a classroom program brought to school children in grades $K-3$ by a law enforcement officer. Several times a years a police officer visits the same classroom and talks with the children. The officer tries to establish personal contact with the children, help them see him or her as a person and as a police officer, and provide an educational program. The officer's goals are to give children an understanding of the roles of law enforcement officials, knowledge of safety rules and sense of responsibility toward themselves, their families and their communities.

- The sections devoted to pedestrian safety in The Idea Book for Planning Your Visits is 13 pages long. It follows the following format:

-- "Purpose" (general instructional goals)

-- "Suggested Classroom Activities (fourteen learning activities, each stated in a sentence or twol

-- Activities sheets with songs, paper and pencil activities, list of safety rules and information about the Helping' Hand Program.

- The police officer also usually provides workbooks for each child in each classroom.

9. Concepts taught:

- Dangers of strangers

- Dangers of strange animals 
- Role of police officer

- Safest route to school

- Hazardous areas (such as railroad tracks; alleys, intersections without traffic lights, vacant houses, areas with moving cars)

- Meaning of traffic light colors and appropriate related pedestrian behavior

- Where to cross the street

- How to cross the street

- Reason for traffic rules

- Safe and unsafe pedestrian behaviors

- Reaction time needed to stop a car

- Where to walk when there are no sidewalks

- Dangers of parked cars

- Dangers of walking after dark

- Dangers of walking in bad weather

- No trespassing.

10. Pedestrian instructions given:

- Stop, look and listen before you cross the street (no emphasis stated on reminding children to look and listen for vehiclesi.

- Look left and right, but don't cross the street until you see the green: light.

- Look all ways before crossing the street.

- Go on the green traffic light and look left and right (only one of several activities mentioned to scan for vehicles), wait on the yellow. light and stop on the red.

- Walk, don't run across the street.

- Take the safest route to school.

- Don't dart out into the road after a ball. 
- Don't walk in alleys.

- Don't trespass on neighbors' lawns.

- Don't talk to, go with, or accept anything from a stranger.

- Run to the nearest house displaying a helping hand if you need help.

- Obey and respect your School Guards and Safety Patrol officers.

- Keep from between parked cars.

- Cross only at corners.

- Don't play in streets, driveways and alleys.

- Wear white or bright yellow after dark.

- Be extra alert in bad weather.

- Obey traffic signals.

- Walk facing traffic when there are no sidewalks (no mention of where near the roadway to walk).

- Obey traffic signs.

11. Teaching methods/materials used:

- Role play in simulated traffic environment (classroom) using KIOS CARRY CARDS

- Map of neighborhood

- Discussion

- Slide show using slides of pedestrians in real traffic environment

- Series of pictures

- Overhead transparencies

- Activities sheets - coloring, following directions

- Songs 
- Poems

- Mobile of signs

- Help of parent in designing the safest route to school.

N.B. Role play is especially emphasized as it allows children to be active and gets them involved. It is of ten suggested that children use the KIDS CARRY CARDS, which are large $8^{\prime \prime} \times 10^{\prime \prime}$ cards each illustrated with a picture of a person, vehicle, street signs or neighborhood scene, etc. It is felt that because $k-3$ children have 1 imited reading skills, these pictoral cards are particularly appropriate teaching aids.

A number of learning activities are stated in the idea, but little or no details are given regarding the lesson objectives, materials needed, sequence to be followed, or desired outcome. The police officers are expected to famfliarize themselves with the developmental capabilities of the children, appropriate teaching methods and ways to carry out the activities.

12. Measurement of children's knowledge and behavior:

- Other than through children's work on the activity sheets and learning activities, no instruments are suggested for evaluating children's knowledge. There are no instruments to measure children's pedestrian behavior, but the program focuses primarily on giving children knowledge of pedestrian safety. 
OFFICER FRIENDLY PROGRAM

Name of program: Officer Friendly Teacher's Idea Book K-4

1. Developers: Hartford Police Department and The Hartford Board of Education.

2. Sponsors: Sears Roebuck Foundation.

3. Date: 1973.

4. Age of children in the program: $K-4$ in public and private schools.

5. Kind of Safety taught:

- Pedestrian

- Bicycle

- Home

- Car

- Fire

- Accident (poisons, etc.)

- Recreational

- Neighborhood.

6. Units reviewed: Entire booklet.

7. Format:

- The primary purpose of the Officer Friendiy Program is to develop in children better understanding of the role of law enforcement officers and more positive attitudes toward police in general. Other purposes of the program include teaching children principles of safety and developing in children a sense of responsibility toward themselves, their families and communities.

- Officer Friendly Teacher's Idea Book K-4 is divided into three basic lessons (after the "Introduction", which states the program's purpose and operations): "Role' of the Policeman", Safety" and "The child as a VIP" (the lesson on responsibilityl. 
- The lesson on safety follows this format:

-- Topic (kind of safety taught and general goal)

-- Teacher preparation (traffic safety vocabulary to be taught to children)

-- Suggest pre-and post-activities

-- Dittos (activities for children) which can be torn out and Xeroxed

-- Test of films and filmstrips and other teaching material.

- Eleven dittos in the trafic safety lessons provide coloring, reading, writing and maze drawing activities. No dittos depict traffic environments, i.e., streets, sidewalks, pedestrians and vehicles. Some safety features--traffic. lights without posts and signs without posts--are shown, but not in a traffic context. One page, for example, shows the tops of eleven signs. other dittos consist of crossword puzzles and reading activities involving phonics and pictures. One page shows a male figure, house, two vehicles, a traffic light and school buflding, each in a separate space, but not incorporated into any meaningful representation of the real traffic environment. Thus, these dittos are highly abstract, particularly so for kindergarten children who may be just beginning. to read and who may not have formed a complete image of all the elements of the traffic scene and their interrelationships in their minds. The people depicted in the dittos are all Caucasian Americans. Police officers are a.lways depicted as male.

8. Concepts taught:

- Crossing streets

- Sidewalk safety

- Safety signs and lights and the meaning of "stop," "go." "walk," "wait," don't walk," "caution," and "yield"

- Playing and running out between parked cars

- Playing near driveways.

- Corisequences when people do not follow safety rules

- Safety rules--only a few are listed

- Safety hazards 
- Where to cross the street

- How to obey the traffic light--what the colors te 11 pedestrians to do

- Names of traffic signs

- How to cross the street.

N.B. Most of the concepts listed above are derived from a review of the activities listed. There is no place where a list of all concepts taught or specific teaching objectives are stated.

9. Pedestrian instructions given:

- Stop at the red traffic light; go on the green; and wait on yellow.

- Stop, look and listen before you cross the street (no mention of what to look forl.

10. Teaching methods/materials used:

- Discussion of traffic safety rules

- Dittos

- Puzzles - crossword

- Role playing safety rules

- Making illustrations and murals of safety rules

- Making posters

- Making charts of safety rules

- Writing stories

- Films and filmstrips

- Putting on a safety play

- Game board simulating an intersection

- Games such as a treasure hunt, "Officer Friendly Says..."

- Interpreting illustrations and putting them in order

- Trips to the real traffic environment 
- Map of traffic environment

- Visit to the classroom by the school Safety Patrol

- Making a model of the traffic light

- Making a graph of accidents in schoo?

- Poems

- Songs.

N.B. These activities are usually described in a sentence without mention of materials needed or how to teach the activity. The police officer can consult the officer Friendly Resource library or other curricula for this information.

11. Measurement of children's knowledge and behavior:

- Toward the end of the booklet there is the "Officer Friendly' Care and Share Survey, "which has fifteen safety questions and blank spaces for answers, spaces for the child's name, school and room numberi and the parent's name and date. Three questions in the survey are directly related to pedestrian safety. The survey assesses children's knowledge of safety rules.

12. Evaluation of this traffic safety curriculum:

- A "Teacher Evaluation of the Officer Friendly Program" is included at the end of the booklet. Other than that, it is not known whether there has been an evaluation of the Hartford Officer Friendly Program. 


\section{Name of program: The Aetna K-2 Pedestrian Safety Program}

1. Developers/sponsors: Driver Education Services, Aetna Life and Casualty.

2. Date: Unknown

3. Age of children in the program: $K-2$.

4. Kind of safety taught: Pedestrian.

5. Materials reviewed:

- "Teach them how...before it's too late!" a brochure, the Coloring and Puzzle book, and the brochure describing the series.

6. Format:

- The Aetna K-2 Pedestrian Safety Program is a filmstrip series with accompanying teacher's manual, response picture cards, and student workbook. Nine filmstrips present "real wor ld" traffic scenes which were filmed from the child's eye level. The film narrator heard in the accompanying cassette tape occasionally asks questions of the students, who can respond either by holding up a response picture card or by talking. (Filmstrip presentations are reported to be easy to stop for class discussions.) The filmstrips, each of which presents a self-contained lesson, are sequentially ordered. The teacher's manuals for unit guides) provide background information for each filmstrip unit and suggest ways to introduce the unit, points to emphasize in discussion, resource materials for follow-up activities and academic areas related to the filmstrip content. While the filmstrip series was designed to supplement not supplant existing state curricula, it has also been developed as a complete unit.

- The Coloring and Puzzlebook contains illustrations of proper and improper pedestrian behavior, a dot-to-dot number activity and visual perception activity which require students to find "hidden" objects. Several pages relate safety rules. Children are expected to color the pictures, fold them, connect dots and in general, follow directions. One or two pictures are on each page. The 
cartoon-like people in the pictures are wearing the up-to-date clothes. The traffic environments shown are quite realistic and also present the child's eye view. Unfortunately, no minorities are depicted. Aside from the illustrations, the remaining activities in this booklet might be quite abstract for kindergarten children who don't know numbers and can't read or write.

\section{Concepts taught:}

- Danger in the traffic environment, dangerous and safe pedestrian behaviors

- Meaning of "pedestrian," meaning of "vehicles, "types of vehicles, parts of the traffic environment where pedestrians and vehicles belong

- Oangers of walking in the street, of parking lots, of driveways, of playing in the street

- How to identify and interpret vehicle sounds to determine location and direction

- How to locate vehicles in the traffic environment by looking and listening

- How to recognize when the vehic les are approaching and departing, knowledge than an approaching vehicle is dangerous. "Fast cars that grow big in a hurry are dangerous." (Coloring and Puzzle Book)

- How to identify fast and slow vehicles and judge when it's safe to go into the street

- Advantages of traffic controls at crossings

- How to cross the street with and without help from traffic controls.

\section{Pedestrian instructions given:}

- Don't play in busy streets or roads; play in the playground.

- Cross the street only if there are no turning vehicles on a green light.

- Other rules as stated in the teaching guides and filmstrips.

9. Teaching methods/materials used:

- Filmstrip and cassette tape

- Discussion 
- Coloring and interpreting illustrations in the coloring book

- Other methods as described in the teacher's guides.

10. Measurement of children's knowledge and behavior:

- The Risk Assessment Inventory (RAI) is used to assess children's knowledge at the end of each of the four units. This diagnostic tool is provided along with the rest of the materials.

11. Evaluation of this pedestrian safety curriculum:

- This program was evaluated in the public schools in Mesa, Arizona, and in the Toddler Inn Development Center in Plainfield, Illinois. It was used in teacher training at the University of Rhode Is land by Dr. Gwenneth Rae, Associate Professor in Child Development and Consultant to this K-2 project. Methods used for these evaluation studies and information relating to the success of the curriculum approach can probably be obtained by writing to:

Driver Education Services $\mathbf{W}-0$

Aetna Life and Casualty

151 Farmington Avenue Hartford, Connecticut 06156. 


\section{5}

PEDESTRIAN PROGRAMS FOR PRESCHOOL CHILDREN OUTSIDE THE UNITED STATES.

In planning and developing a preschool pedestrian safety program in this country, it is fortunate that there are many foreign models to consult. A number of countries have long-established traffic safety programs for preschool children. The purpose of this chapter is to describe such programs. Specifically, this chapter attempts to:

- Identify countries where pedestrian programs for preschoolers exist

- Present an overview of various program approaches used

- Summarize the concepts taught to preschoolers

- Review the pedestrian instructions given

- Describe the teaching methods/materials used

- Indicate the type of information parents of preschool children receive, and

- Summarize the contributions made to the field of preschool pedestrian safety education.

As in Chapter 4, the first part of this chapter is an overview of programs described in depth at the end of the chapter. The countries with preschool pedestrian programs identified in this report are those countries which were identified in the research literature as having programs. This list of countries is by no means all inclusive, as there was no attempt made to contact every foreign country. Rather, those countries referenced in the literature were contacted efther through 
their embassies or through their representatives to the OECD Special Research Group on Pedestrian. Safety. The following countries were contacted initially in 1979:- " Australia, Canada, Denmark, Finland, France, The Federal Republic of Germany, Japan, Luxemburg. The Nether lands, New Zealand, Norway, Sweden, and The United Kingdom. The pedestrian program materials sent by these countries form the basis of this overview of pedestrian programs for preschool children in foreign countries.

Who develops, evaluates, and sponsors these programs and program materials? As in the United States, many organizations within the public and private sector have contributed to the development of pedestrian safety education. The Australian Department of Transport, which develops and distributes materials to schools and families, is an example of a branch of a national government which is responsible for initiating pedestrian safety education. State agencies such as Innenministerium Badin (a German State) and local government agencies such as the local Prefectures in Japan also are known to implement programs and develop materials. Private organizations such as ADAC (the automobile club of The Federal Republic of Germany), Jugendwerk der Deutchen She 11 (Royal Dutch She 11011 Company in the Federal Republif of Germany), Yletsradio (the Finnish Broadcasting Company), and Kindai-Keisatsu sha (a publishing company in Japan) have been instrumental in the development of pedestrian safety education. Universities such as the University of 0 s 10 in Norway, the University of Tempere in Finland, and the University: of Reading in England have also been responsible for developing and evaluating pedestrian safety programs.

1/ Countries contacted in the second mailing (early 1980) include: Algeria, Austria, Belgium, Brazil, Chile, Dahomey, Egypt, Gabon, Greece, Hungary, Israe 1, Ivory Coast, Jordan, Kuwait, Poland, Portuga 1, Senega 1, Soudan, South Africa, Spain, Switzerland, Tunisia, Turkey, Yugoslavia, and Zaire. Regrettably, these materials cannot be described in this Literature Review, as they were not received in time for inclusion in this document. 


\section{TYPES OF PRESCHOOL PEDESTRIAN PROGRAM MATERIALS}

All of the foreign pedestrian programs and program materials identified in this Literature Review share a common approach: all programs appear to emphasize the parent's role as the primary and most important teacher of pedestrian safety. Considerable effort is made towards informing parents about child pedestrian accidents, children's behavior in traffic, children's capabilities, pedestrian rules, and appropriate instructional techniques.

Aside from this overriding similarity of purpose, the programs are different in approach. Judging from the program materials and program descriptions received, there are four types of pedestrian programs for the preschool age group implemented in foreign countries. A description of each type follows.

\section{I.A. School Approach}

Most of the school-based pedestrian programs have a formal or an informal parent component. The Traffic Education Programme for Preschool Aged Children and Children Starting School study undertaken in Finland is an example of a school-based program with a formal linkage between school and home. In this program, each of the teacher's lessons in the classroom is followed up during the succeeding days with parent instruction in the home. Children view slides in the classroom and later practice those same instructions in traffic under their parents' guidance and supervision. Programs with an informal parent component include England's Children in Traffic series approach, which utilizes parents as teachers on an occasional basis for on-street instruction of children, and an Australian school program, which according to our understanding, sends parents brochures to inform them about their children's pedestrian program at school. Parents in each of these three programs are encouraged to reinforce concepts being taught at school.

As might be expected, the materials in these programs vary in scope and approach. Road Safety: A Manual for Teachers, used in Australian schools, is more of a general guide than a detailed curriculum manual. It presents pedestrian safety rules and explains their rationale but does not provide 
lesson plans. The Traffic Education Programme for Preschool Aged Children and Children Starting School manual, however, which is used by both teachers and parents, is very detailed and specific. It tells parents and teachers exactly what to say and how to say it, and it utilizes two clearly defined teaching methods-sildes and on-street traffic training. The children in Traffic series presents sequential detalled lesson plans with illustrations of examples of on-street training and follow-up activities in the classroom. This book series also provides teachers with background information about children's behavior: in traffic, children's traffic accidents, ways to involve. parents and community members in the program, and guidance on when to introduce new concepts.

\section{I.B. Television Series Approach}

The United Kingdom has decided that an effective way to involve the whole family in pedestrian safety education is to institute a pedestrian safety television series for parents and children and to provide them with related print materials. This approach was initiated with two television spots called Lesson for Life in 1975 and two commercials in 1976. Parents were sent print materials to further clarify and reinforce the information presented in a Lesson for Life, but no nonbroadcast materials were sent in conjunction with the television commercials. The television approach has the advantage of reaching wide audiences and developing a greater level of awareness of children's difficulties in traffic. Further research may reveal additional television programs for preschool children and parents that have been instituted in foreign countries.

\section{C. Print Materials Approach.}

Brochures, booklets, and books for parents and children have been developed by several public and private organizations within various foreign countries.

Brochures are usually aimed at parents; but the illustrations in many brochures can be used for teaching chlldren principles of pedestrian safety. The primary aim of the brochure is to increase parents awareness of the 
"problem," inform them about correct pedestrian procedures, and suggest ways they can teach pedestrian safety to their children. As brochures are short, information presented has to be very concise but attention-getting. Organizations within the Netherlands, Switzerland, and Australia have produced such brochures for parents of young children.

Book lets have been developed by two states in the Federal Republic of Germany. Both booklets provide colorful photographs depicting model pedestrian behaviors and model teaching behaviors for parents to use when instructing their children in traffic. These photographs depict both parents and children as role models. Information about children's traffic accidents, reasons for their vulnerability in traffic, and information about their modes of learning are provided as we11. Illustrations in these booklets can easily be used in teaching children correct pedestrian behaviors.

The books reviewed have been developed explicitly for children. ADAC's two books for the preschool age group are designed to encourage children to make observations and draw conclusions. Sicher zur Schule encourages children to predict consequences of incorrect pedestrian actions, while Die Vorschule der Verkehserziehung provides children with opportunities to develop perceptual abilities and recall how parts of the traffic environment fit together. Both books have large, colorful illustrations with few words on each page.

\section{D. The Children's Traffic Club Approach}

There are three types of children's traffic safety clubs: (1) the club concept as originated with Norway's Children's Traffic Club, (2) the meeting club approach, as advocated by The Tufty Club developed by The Royal Soclety for the Prevention of Accidents (RoSPA) in the United Kingdom, and (3) the audio-visual modular club approach as innovated by Finland.

The concept of the Children's Traffic Club which Trygg Trafikk developed in Norway is similar to that of the college correspondence course. Families are sent packets of written materials at regular intervals over the course of several years, and children complete an organized sequential course of study 
over a period of time. The difference between the Children's Traffic Club and the correspondence course lies in club membership. The children's traffic clubs in Sweden, Denmark, Norway, Germany, Luxemburg, and the United Kingdom utilize various techniques to make children and parents feel that they belong. Badges, books addressed directly to the parents and children, and regular correspondence initiated by both parents and the club are some of those techniques. While the club materials vary somewhat in terms of the type of information given to parents, the children's materials in these clubs are similar in that most contain fllustrations, puzzles, records, panoramas, and stories: All the clubs emphasize on-street traffic training.

The meeting club approach appears to be similar to that utilized in the Boy Scouts or Girl Scouts in the United States. Parents are responsible for initiating, organizing, and running the clubs. The concept of using such an approach in a children's traffic club originated with the Tufty club in 1961. 1/ In the United Kingdom and Japan where such meeting clubs presently. exist, parents bring their children to a designated meeting place and institute a classroom.type of program. In some instances they institute group training in real traffic as well. In addition, parents continue the training on an individual: basis with their children in the home. It seems that these clubs offer parents support, intellectual stimulation, and other role models. Children appear to benefit from seeing other children trying to learn to become safe pedestrians. They also seem to benefit from the variety of teaching methods which are carried out in both a classroom and home training program.

Finland is the innovator of a new type of children's traffic club. Lifkenneturva (the Central Organization for Traffic Safety) and Yleisradio (the Finnish Broadcasting Company) have developed a club which utilizes television and parents as teachers. The Noppa program is a television series consisting of seven 25-minute programs, which both parents and children.can

1/ Actually, the Tufty Club advocates both the club approach mentioned here and an individualized home training approach simflar to that used in Norway's Children's Traffic Club: 
enjoy. In addition, five- to eight-year-olds receive Ekaluokalaisen Lifkennevihko (A Traffic Book for a First Grader), which parents and children read together and use as a guide for on-street pedestrian training. The other program in Finland's Children's Traffic Club, Pim Pam Pelista Pois, also utilizes of a series of television programs and a related book for parents and children. This club approach, instituted first in 1976, may be incorporated into other clubs, as so many parents and children spend time watching television and as they enjoy reading books together.

Finland's Children's Traffic Club has also gone beyond other clubs in educating parents about their role as creators of the traffic environment. Apparently this emphasis is being incorporated into other club booklets.

\section{CONCEPTS TAUGHT TO PRESCHOOL CHILDREN}

While there are apparent differences in the concepts taught to preschool children in various foreign pedestrian programs, one general trend is the same: children begin learning about the most basic parts of the traffic environment--streets, sidewalks, and curbs--before they are expected to learn about complex traffic situations such as controlled and uncontrolled intersections and abstract parts of the traffic environment such as traffic signs. Children are generally not expected to evaluate an entire traffic system to decide upon the safest route to school until they are into elementary school and age six or older. In children's traffic club materials, one or two concepts are usually presented per booklet rather than overwhelming children with many concepts at once. In many clubs new concepts are presented every six months. Generally, as children get older, they are taught concepts such as where to walk on snowy roads, how to walk safely in darkness, and where to play in snowy weather. In summary, whether pedestrian programs begin with three-year-olds or five-year-olds, they begin by introducing children to the simplest concepts and gradually build on them in a sequential manner.

\section{PEDESTRIAN INSTRUCTIONS}

For the most part, the pedestrian instructions taught to children are the same as those described in programs in the United States. The few differences 
apply to the following type of instructions and/ori decisions: where to walk when no sidewalk exists; where children should first be taught to cross the street; and how parents should: interact with their children when walking in traffic. In the United Kingdom and Australia where vehicles drive on the left side of the road; children are taught to look right-left-right for vehicles before crossing the street and to walk on the right shoulder of the road when no sidewalk exists. In the forefign countries whose materials we reviewed, children are taught to cross the street mid-block at pedestrian crossings before they are taught to cross at pedestrian crossings at the corner: It appears that pedestrian crossings are located mid-block more frequently in foreign countries than in the United States. In some respects, crossing the street at pedestrian crossings at mid-block may be easier for young children, as they do not have to be aware of as many directions and as many vehicles at the same time as they would were they crossing at pedestrian crossings located at intersections. On the other hand, when cars park very close to pedestrian crossings, childrens' and drivers' ability to see each other is reduced.

The pedestrian instructions pertaining to parents' behavior with their children while in traffic are more explicit in foreign countries pedestrian materials than in most materials from the United States. For example, parents are told always to hold their children's hands while in traffic, to place themselves between their children and the road, to squeeze their children's hands while stopping at the curb, and to use concrete objects in the traffic environment to orient children so that they will follow the proper procedure for scanning for vehicles.

For the most part, pedestrian instructions in foreign countries are phrased simply and positively. Children are taught what to do rather than reminded what not to do. The language used in pedestrian instructions is very simple and concrete. For example, rather than telling children to "stop" on red and "watt" on yellow, chlldren are told that both mean "stop." Rather than using words such as "left" and "right," parents are told to substitute words such as "one side" and "the other side." There is an evident effort to make the instructions developmentally. appropriate for the children. 


\section{TEACHING METHODS}

On-street traffic training is the teaching method most strongly advocated in foreign traffic programs. All of the other activities--books, television, slides, discussion, puzzles, stories, songs, artwork, and role-play--serve to reinforce and clarify concepts learned in traffic training. A strong message given to parents is the role that they as models and teachers play in teaching their children attitudes toward traffic safety, pedestrian habits, and knowledge of the traffic environment. Parents are encouraged to communicate reasons for their actions and decisions in traffic to their children when they are together in traffic. Parents are also encouraged to be demonstrative when they model behaviors such as looking for vehicles. They are told to ask their children questions about the traffic environment and pedestrian behavior in order to find out the depth of their children's understanding. They are also encouraged to let children make occasional traffic-related decisions when they are together in traffic and show them in an informal testing situation le.g., how to cross the street) that they know the pedestrian rules and can demonstrate what they know. Parents are advised never to let preschool children play or walk outside unsupervised and never to let go of their children's hands in traffic.

Children's materials used in the children's traffic clubs are considered an important part of instruction. It is clear from the extensive use of artwork (both color photographs and color drawings) and the use of records, puzzles, and cardboard traffic panoramas that program developers and sponsors went to considerable effort and expense to develop exciting, colorful, and developmentally appropriate materials.

Illustrations in the children's club materials depict traffic environments with detail and realism. For example, traffic signs are always shown in the context of a traffic environment. Children who are scanning for vehicles are usually shown in a wide-angle view drawing of the road so that it is clear that they are looking in each direction for vehicles. Shading, color, and details such as shrubs, fences, and grass are used to differentiate parts of the traffic environment such as sidewalks and streets from each other. 
Illustrations also are created with a certain amount of idealism. For example, correct pedestrian behaviors are modeled by young children and parents. Children are usually shown accompanied by parents, and parents are of ten shown giving their children traffic instruction. The pedestrians shown are wearing up-to-date clothing and look happy about obeying pedestrian safety rules:

The materials make extensive use of color. As there is usually onty one large fllustration per page or per two-page spread, the materials are also easy for children to "read" and attractive to the eye.

Considerable thought and effort has been made to create materials that will be meaningful and comprehensible to children. Most of the lllustrated traffic situations are drawn or photographed from the side or child's eye view. Overhead views of the traffic environment are not used until elementary school. Parents are also encouraged to help children see the relationships. between parts of the traffic environment and pedestrian behaviors in the book with the real traffic environment and their own behavior in traffic when they are out in traffic together.

\section{INFORMATION GIVEN TO PARENTS}

As the parent's role in children's pedestrian safety education is considered so crucial, great care is made to provide parents with sufficient information about children's behavior in traffic, children's capabilities, pedestrian safety rules, methods for teaching pedestrian safety (on-street traffic instruction as we 11 as how to use the club materials), and the importance of the models which parents present to children. For example, parents are given specific guidelines for what their children can and cannot be expected to do in traffic. Parents are given child development information so that they can better understand why children are at risk in traffic, how children learn pedestrian safety, and how children learn and develop in general. As mentioned earlier, Kilpeli, a booklet from Finland's Traffic Safety Club, informs parents about children's need to play and, consequently, their need for a safe traffic environment. Parents in this club are also shown examples of how the traffic environment can be made safer so that 
children and vehicles are less likely to be in conflict. Thus, most children's traffic materials are as much directed to parents and teachers as they are to children.

\section{SUMMARY}

In conclusion, pedestrian programs in countries outside the United States and children's traffic clubs in particular have contributed much to the field of pedestrian safety education. They have developed a model pedestrian safety education approach that truly utilizes parents as children's primary and most important teachers of traffic safety. In addition, they have led the way in designing curricular materials that are creative, innovative, and instructive for young children and enjoyable for parents and children to use together. 
PEDESTRIAN PROGRAMS FOR PRESCHOOL CHILDREN

IN COUNTRIES OUTSIDE THE UNITED STATES 


\section{AUSTRALIA}

Name of program: "What We are Teaching about Road Safety"

1. Issued by: The Office of Road Safety, Department of Transport, Australia, in cooperation with the road safety authorities of the Australian States and Territories.

2. Date: The brochure appears to be recently issued because the photographs reveal modern housing and pedestrians wearing current fashions.

3. Target audience: Parents of young children.

4. Kind of safety taught: Pedestrian.

5. Format:

- This eight-sided brochure contains recent black and white photographs of two children in traffic by themselves and two parents walking with their children. Some photographs show sequences. For example, one shows what the traffic light and "Walk" lights look like and appropriate pedestrian behavior when the light is green, red, and yellow. It shows two children waiting on the sidewalk on red and yellow and walking across the street on green. Because the photographs are black and white, one has to know the special position of the lights and read the "Walk" - "Don't Walk" signs to ascertain what is going on. Another sequence shows children looking right-left-right from the curb but does not show the road or vehicles.

- Whenever parents are shown walking with their children, they are always holding their hands. The parent walking with the child on the sidewalk is walking between the child and the road.

- The brochure is addressed "Dear Mummy and Daddy" and informs parents that "We" (their children) are learning about road safety. The numbers of children's accidents are quoted and parents are asked to point out dangers of traffic to their children and model correct behaviors. Following that page are six pages, each of which teaches a pedestrian safety principle.

6. Concepts taught:

- Reasons not to play in the road; safe places to play

- Meaning of traffic light signals, "Walk - Don't Walk" lights, and policeman's signals 
- Where to cross streets; to listen as well as look for cars

- The importance of having an adult accompany children and children.knowing their names and addresses in case they get lost

- How to scan for traffic and cross the road

- What a footpath is for; what to do when there is no footpath (sidewalk).

7. Pedestrian instructions given:

- Play in yards, parks, or other safe places.

- Never chase a ball or playmate onto the road.

- Don't ride a tricycle, scooter, or billycart on the street.

- On the red signal light, stop. On the yellow signal light, wait. On the green, walk. if the way is clear.

- Obey the police officer's hand signals. (It is explained that one hand signal means "stop" and the other means "walk.")

- Cross busy streets at traffic lights, marked crossings, where a police officer or warden is present to stop traffic. When none of these conditions exist, choose a straight part of the road to cross.

- Children should stay close to their adult escort when walking on busy streets.

- To cross the street: stop at the curb, look right-left-right, and if the road is clear, walk straight across, while keeping a lookout for cars. Do not run.

- Walk on the sidewalk (footpath).

- When there is no sidewalk, walk on the right side of the road facing oncoming traffic. 
AUSTRALIA

Name of program: Children's Highway Code

1. Produced by: The Office of Road Safety, Department of Transport, Australia, in cooperation with the road safety authorities of the Australian states and territories.

2. Date: 1979.

3. Age of children in the target audience: Beginning school-age children.

4. Kind of safety taught:

- Pedestrian

- Bicycle

- Car Passenger

- Bus.

5. Format:

- This 18-page booklet recites pedestrian safety rules and provides related 1 llustrations. The fllustrations are in cartoon format. The lliustrations of the environment are sketchy. Sometimes the road is painted a russet hue but at other times, the street is represented by a line, or not represented at all. For example, one sequence of pictures shows the rear view of two children standing still, looking right-left-right and then walking, but no street context or vehicle. Another picture shows a crosswalk but no street. The majority of pages teach pedestrian safety.

6. Pedestrian instructions given:

- When a sidewalk exists, walk on it. Walk on the left side of the sidewalk.

- When: there is no sidewalk, walk on the right facing oncoming traffic.

- At night, carry a light or something white.

- To cross the road, stop at the curb, look right-left-right, and if the road is clear, walk (don't run) straight across, looking and listening for traffic as you cross. 
- Cross the road at pedestrian crosswalks, at traffic lights, where police officers are directing traffic, or at the corner.

- The red traffic light signal means "stop." The yellow means "wait." The green means "go."

- Never suddenly run out into the road.

- When crossing one-way streets, look in the direction of approaching vehicles. Don't leave the sidewalk until it is safe to cross.

- Be extra careful when you cross near parked vehicles.

- When it's raining and you want to cross the street, fold your umbrella when you cross so that you can see vehicles.

- Younger children should be held firmly by the hand when crossing the road.

- Play in the park, in the yard, or in playgrounds, never on the road and preferably not on the sidewalks.

- Don't chase a ball or friend into the road. 


\section{AUSTRALIA}

Name of program: "Hello, I'm Hector Cat ..."

1. Produced by: The Office of Road Safety, Department of Transport, in cooperation with the Road Safety Authorities of the Australian States and Territories.

2. Date: 1979.

3. Intended audience: Parents of young children.

4. Format:

- This six-sided brochure has cartoon drawings of pedestrians and drivers and a sketchy aerial view drawing of intersections and streets. The characters are chubby and slightly humorous. The tone created is humorous to provide a balance to the serious tone of the lesson.

- In the brochure, parents learn facts about children's pedestrian accidents and steps parents can take to prevent their children from having such accidents.

5. Information presented:

- The time of day that children have pedestrian-auto accidents

- The need to teach children the safest route to school

- What children are doing when they are hit (running)

- Where child pedestrian-auto accidents occur

- Tips for increasing children's safety when they are driven to school

- That children need to be taught the basic parts of the traffic environment such as traffic lights

- That children must be taught pedestrian safety skills (a few skills are discussed)

- That children's pedestrian performances are erratic

- The importance of dressing children so they are visible

- Rules for buses, trams and trains

- How to cross the road

- Where children should play

- The police officer's role in traffic. 
AUSTRALIA

Name of program: Road Safety: A Manual for Teachers

1. Compiled by: The Commonwealth Department of Transport in cooperation with the traffic and road safety authorities of the Australian States and Territories.

2. Printed by: Wilke and Company, Ltd. in Clayton, Victoria.

3. Date: 1976 .

4. Age of children in the program:

- Junior Primary School students (under ejght years)

- Senior Primary School students

- Junior Secondary School students

- Senior Secondary School students.

5. Kind of safety taught:

- Pedestrian

- Bicycle

- Signs and Signals

- Driver.

6. Material reviewed:

Material for the youngest age group.

7. Format:

- This 93-page teacher's manual is divided into chapters according to age group and according to three topics-cycling, signs and signals, and teaching aids.

- The section for Junior Primary School students consists of 15 sections--called stages. The stages basically repeat the same traffic rules taught in the "Children's Highway Code" and emphasize issues that teachers should discuss when teaching the rules. While the book let mostly consists of listings of traffic rules, a few suggestions of preferable teaching methods are given.

- The traffic rules are illustrated with cartoons.

8. Concepts and pedestrian instructions given:

- The same as those in the "Crifldren's Highway Code." 
DENMARK

Name of program: Danish Children's Traffic Club

1. Sponsor: Danish Road Safety Council.

2. Date: Since 1969 .

3. Age of children in the program: three to seven years.

4. Kind of safety taught: Pedestrian.

5. Club operations:

- Essentially this Children's Traffic Club is a correspondence course. Starting at the third birthday, and ending at age six-and-a-half children receive club materials every six months. They receive eight packages in all, plus eight surprises for completing training each time. The surprises are stickers which are attached to the children's traffic panorama. It is our understanding that Radet For Storre Faerdselssikkerhed (a branch of the Danish Government) assumes all club operating costs.

6. Materials reviewed: All materials in the Children's Traffic Club.

7. Format of the materials:

- The materials consist of seven chapters, or units, of separate $12^{\prime \prime} \times 7^{\prime \prime}$ pages and a small booklet which are inserted into a $12^{\prime \prime} \times 7^{\prime \prime}$ four-ring red binder. Children also receive two large, colorful, glossy panorama sheets with stickers that adhere to them, a spoon imprinted with the Traffic Club insignia, and a plastic car. Children receive a unit every half year, except for their four-and-a-half year when they receive a book let. Thus, the contents of the book expand as the child grows older and learns more concepts.

- Each unit consists of the following:

-- Letter of introduction to the chapter which includes: some information about the characteristics of that age child in traffic, (2) general tips on teaching/giving on-street training, (3) concepts emphasized in that chapter, (4) instructions on the problem or test to give the child once training in that chapter is completed, and (5) a reminder to mail in the prize card. The prize card indicates that training has been completed and alerts the club that it's time to send back to the child a prize sticker, which belongs on the large glossy panorama mentioned earlier (separate from the paper panoramas contained in each chapter). 
-- Several pages of text on the left-hand side, each complemented by an tllustrated page on the right. The text has two sections, one addressed to the parents ("To Mom and Dad") and one which is read to and addressed: to the child personally.

-- A paper panorama with paper cut-out pieces and some other types of learning activity.

- The illustrations in the book are very colorful and varied. One type of illustration is a drawing with every inch of space colored in with a bright color: A11 elements of the traffic environment are very recognizable because of the level of detal1. For example, the stdewalks even have white $r$ ims and large squares of concrete. Grass, flowers, trees, buildings, etc., line the sidewalks and the streets and are a separate color than the sidewalks, thus clearly differentiating these two parts of the traffic environment. The people in these drawings look like cheerful, colorful dolls, almost like cartoons. All in all, these drawings are bright, sunny, and colorful, easy to "read" for a child. A second type of fllustration used is the photograph--black and white as well as color. Inner city, suburban, and rural traffic environments, different kinds of vehicles, and pedestrians in different kinds of traffic situations have been photographed. The book let for the four-year-old consists of a sequence of black and white photographs which tell the story of a four-year-old who crosses the street despite bullies on her block.

8. Concepts and skills taught to three-year-olds:

- To distinguish between the sidewalk and road

- That they, as pedestrians, should walk on the sidewalk

- Where to walk if no: sidewalk is available

- To distinguish between adults' and children's vehicles

- Different kinds of safe outdoor play areas which they can and should use:

9. Concepts and ski1ls taught to three-and-a-half-year-olds:

- How to tell which direction a vehicle is going

- That cars move in different directions and occupy different spaces on the road

- To stop at the curb and look both ways for drtvers before crossing a street with an adult

- To distinguish between the colors red, yeillow, and green. 
10. Concepts and skills taught to four-year-olds:

- To cross the street which parents have been teaching them to cross by themselves

- To "read" and interpret traffic lights

- To stop and look both ways until no drivers are coming before crossing the street.

11. Concepts and skills taught to four-and-a-half-year-olds:

- Mostly a review of all that is taught before

- Consequences of safe and unsafe traffic behaviors.

12. Concepts and skills taught to five-year-olds:

- To learn about other pedestrians: children, adults, and old people and their difficulties in traffic

- To point out pedestrian mistakes and explain why people making mistakes might be behaving as they do and why an accident might occur

- To begin to judge vehicle distance, speed, and braking power (making very simple judgments, i.e., near vs. far, fast vs. slow)

- To understand vehicle turn signals.

13. Concepts and skills taught to six-year-olds:

- To identify the dangers of different kinds of roads, curves, hills, etc.--and point out two dangers, in six pictures showing different traffic situations

- To cross at intersections, looking sideways as well as behind and forward for drivers

- To determine, with parents, the safest route to school and make a map of the route.

14. Pedestrian instructions given:

- Walk on the sidewalk. Vehicles belong on the street.

- If there is no sidewalk, walk on the left side of the sidewalk on the edge where the grass or dirt starts so you can see oncoming vehicles.

- Watch to see where vehicles are going--whether they're coming towards you, going away, etc. Turn your head and really look at them, 
- When someone calls to you across the street, stop at the curb, look in both directions, and wait until you see no more cars. If no more cars are coming, walk strajght across the road.

- When you want to cross the street, always use a crosswalk when one exists.

- At the traffic light, stop when the light is yellow or red. When the light is green, watch in: one direction and then in the other direction to see if the vehicles stop. If they do, you can cross the street.

- Don't expect vehicles to be able to stop suddeniy.

- Pay attention to other. walkers so you don't bump into them.

- Look for blinking signal lights on vehicles and arm movements on bikes and motorcycles. to tell which way they are moving.

- Watch to see which way and how fast vehicles are coming.

15. Teaching methods used:

- Reading the book lets and interpreting the illustrations

- Parents modeling correct pedestrian behaviors in front of their children and explaining step-by-step what they're doing and why

- Parents instructing children in correct pedestrian behaviors

- Parents and children doing the puzzles and activities in the units (activities such as placing the cut out pedestrian in the proper spot on the traffic panorama)

- Parents and children observing the real traffic environment and identifying different safety features and behaviors

- Parents giving children problems to solve (e.g., crossing the street correctly five times in order to past the "test" and mail away the prize card)

- Introducing concepts sequentialiy, so that easier concepts are tackled first

- Parents asking children questions about traffic situations and listening to how they answer the questions

- Teaching a child how to cross the street by teaching him/her initialiy at the same familiar spot on the same street

- Repeating the same on-street pedestrian lesson many times. 
16. Measurement of children's knowledge and behavior:

- In order to send away their prize card to receive a prize once a lesson is complete, children are "tested"--asked to solve a problem, either behavior-oriented or knowledge-oriented. For example, after one unit, a child has to prove that he/she can cross the street safely five separate times. 


\section{DENMARK}

Name of program: Children on the Roads (a campaign to increase children's safety in traffic)

1. Sponsor: The Danish Road Safety Council (under the auspices of the Government Commission on Road Safetyl.

2. Date: 1979.

3. Target audience of the campaign: Children and parents.

4. Kind of safety taught:

- Safe driving

- Pedestrian safety.

5. Program materials reviewed: None.

6. Program format:

- A television series, consisting of eight television shows, has been designed to remind parents of safe driving rules and to help them instruct their children in the principles of road safety.

- Parents also receive a program textbook called children on the Roads, which reinforces and expands upon the concepts presented in the television series.

- The series informs parents why the accident rate for young children is so high and describes children's behavior in and near traffic. The characteristics of children ages three, four, five, six, and seven are described, so that parents can form a better understanding of children's needs for supervision and instruction. Parents are advised that children ages three and four should always be accompanied by an adult when they are out in traffic. Parents are told that while ifive and six-year-olds can walk independently and safely in trafific, in many circumstances they have difficulty assessing dangers in traffic when they are in the company of other children and when they are required to react to various stimuli at once. However, by the age of seven, children are more or less able to walk safely on their own, according to the series.

N.B. The above information was derived from Gallienne and Pansard, (1979). 
FEDERAL REPUBLIC OF GERMANY

Name of program: Die Vorschule der Verkehserziehung

1. Authors: Horst Nowak, Heinrick Wuhrer, Veronika Neubauer-Zacharias ADAC Ver lag.

2. Sponsor: ADAC Verlag.

3. Date: 1970.

4. Age of children in the target audience: Kindergarten.

5. Kind of safety taught: Pedestrian.

6. Material reviewed: The booklet was loaned, but no translation has been done. However, it is possible to gain some insight into the materials just from the pictures.

7. Format:

- This book appears to be designed for parents and children to read together. There is very little writing in the book: the only texts are on the first and last two pages. The first text appears to be an introduction, while the last two pages appear to contain directions for parents about how the book is to be used. otherwise, there are only short phrases such as "Links and rechts" (left and right) for a two-page spread.

- The first two-page spread has three rows of balls, each which has its own color and letter or number. Lines are drawn connecting the blue balls. It appears that this two-page spread is an activity designed to help children learn and match colors.

- The next two-page spread shows traffic lights and a series of boxes, each of which has a colorful picture of an object (e.g., an elephant, star) or a drawing with no color.

- The next spread has five lines of symbols which are large on the top row, smaller on the next row, smaller still on the third row, and tiny on the bottom row. As the same symbols appear in each row, it appears that the purpose of this activity is to have children match symbols and notice that the symbol retains the same shape regardless of its size.

- The next three two-page spreads appear to focus on leftright concepts. The left hand is drawn on the left page and the right on the right. Two of the spreads in this series have pictures of other objects shown with left and right hands. All of these objects are objects which move or point left or right. 
- The next two-page spread appears to focus on cause and effect concepts. Each picture shows a person doing something that will have a definite consequence. For example, a dog and a boy on a scooter are about to collide. A lion is about to jump through a hoop. A fish is about to eat a worm and be caught by a fisherman. The three pictures on each page are very colorful.

- The last series of pages in the fllustrations contain puzzles. There are five pages, each filled with pieces from different puzzles. In other words, there are five puzzles, in total, which can be fit together. The child needs to cut up the pieces and fit them together. When assembled, the puzzles depict traffic scenes. 
Name of program: Sicher zur Schule

1. Authors: Carsten Feeser, Barbara von Johnson - ADAC Verlag.

2. Sponsor: ADAC Verlag.

3. Date: 1971.

4. Age of children in target audience: two to six years.

5. Kind of safety taught: Pedestrian.

6. Material reviewed: The book was loaned, and parts of it were translated into English.

7. Format:

- The first part of the book is a story intended for parents and children to read together. The very last pages consist of a text that advises parents how to teach pedestrian safety and two pages of illustrations of vehicles, sizes, signals, police officers, houses for children to cut out and play with.

- This children's book has one of the more unusual formats seen in children's pedestrian safety booklets. Each two-page spread depicts a lively androgenous character named Andreas doing something dangerous in traffic. If one turns a half-page that fits in the middle of the two-page spread to the left and pushes it back, the consequences of Andreas' incorrect pedestrian behavior are shown. For example, one sees a scene in which Andreas is playing with a ball on the sidewalk on both pages and next, a scene with the ball rolling under a car in the two-page spread that is created by turning the half-page.

- The story unfolds as a school child sits down in the bus and Andreas, a kind of imaginary playful creature, appears. Andreas accompanies the school child on the route to school and breaks every pedestrian rule along the way:

-- Darts out into traffic (into the middle of the street the minute he gets off the bus)

-- Plays with a ball on the sidewalk and is inconsiderate of an older pedestrian

-- Doesn't pay attention to traffic and what he/she is doing while he/she rollerskates into the road--in front of a bicyclist 
- Fails to stop, scan for traffic and wait for cars to stop at a pedestrian crossing

-- Runs out into the intersection into traffic even though the "Don't Walk" light is flashing

-- Grabs onto the outside of the bus and rides away as the school child goes into his school.

The school child models only correct pedestrian behaviors and is safe, while Andreas models incorrect behaviors and gets into dangerous situations.

The illustrations in the book are very colorful and bright. The traffic environment is not shown. with a lot of realism, but color and shape are used to set off different parts of the traffic environment. For example, the road is always a light gray, while sidewalks are white, and buildings, vehicles, and pedestrians are drawn in bright colors. The buildings look like a collage of colored rectangles. People are drawn like cartoon characters. The main characters, Andreas, the school boy, and the drivers or pedestrians whom Andreas darts into, are depicted: in. greater detail than the minor characters. Minor characters are ink sketches, while the main characters are drawn in color and more detail. Crosswalk traffic lights, walk lights, and pedestrian signs are also drawn in color and placed in environmental context. 
FEDERAL REPUBLIC OF GERMANY

Name of program: Schritt fur Schritt mehr Sicherheit

\section{(Step-by-Step - More Safety)}

1. Sponsor: Nordrhein - Westfalen e V. (one of the states in the Federal Republic of Germany).

2. Date: 1977.

3. Kind of safety taught: Pedestrian.

4. Age of children taught: Children in the illustrations appear to be in preschool and elementary school.

5. Materials reviewed: A copy of the booklet in German and a brief summary of its contents in English.

6. Format:

- This manual has been written to serve as a guide for parents. It shows them how to teach their children to behave correctly in traffic. The manual explains that children in street traffic are particularly exposed to danger because their faculties of seeing, hearing, moving, and reacting are not yet fully developed. Concepts such as left, right, quick, slow, in front, behind, etc. still cause them great difficulties. In order to better acquaint their children with traffic, parents are told to proceed on a "step-by-step" basis. After the child has fully comprehended one step, he/she will be eager to learn the following steps. As children learn most easily by imitation, parents are told to give their children a good example in street traffic all the time: Parents are also advised to cooperate closely with kindergartens, which are always in need of helpers in traffic training, and to support street crossing patrols and policemen.

7. Suggestions for Parents:

- Step No. 1:

-- Always give your child a good example. After all, you cannot expect your child to do correctly what you did wrong.

-- Always walk on sidewalks. Cross the street only at intersections and on crossings provided for pedestrians. 
-- Never cross the street. when the light is red even if there is no traffic.

-- Never show your child that you are in a hurry.

-- The child should be able to recognize traffic violations. Make your child aware of them.

-- Always explain your actions in a low voice. The child will understand that aduits think before acting. Example: "The light is red. We still have to wait." At pedestrian crossings: "Will the cars stop? Yes, they have seen us; we can go now:"

- Step No. 2:

-- Have one hand free all the time so as to be able to assist your child. In unusual situations, your child should always feel safe because of your presence.

-- If you have too much to carry, go twice, but never allow your child to walk behind you.

-- In the stores, your child should stay close to you all the time.

-- Do not scold your child while walking with him. Defiant children will of ten tear loose from their parents.

-- Talk to your child even if you have other things on your mind: The child should know that he is walking with his parent(s).

Step No. 3:

-- Teach your child how to behave in certain situations.

-- Always have your child walk next to you on the side more distant from the street.

-- Always make a short stop at the curb so as to demonstrate to him that you have come to a borderline, even if you intend to cross the street immediately.

-- Do not go too close to the curb as your child might be hit by a passing vehicle.

-- If possible, cross the street only at marked crossings. In their absence, select the safest site. 
-- Before crossing the street, always stop for a short while even if you have a green light. In this manner, you create a dividing line in the child's subconscious between the sidewalk (safe) and the street (dangerous). When the light is green, make a habit of looking to the left and to the right while crossing.

-- Before crossing the street, look demonstratively to the left and right.

- Step No. 4:

-- Acquaint your child with his surroundings, because even in street traffic, your child will consciously be aware of his environment.

-- Occasionally, have your child tell you the colors of items with which he is familiar. For instance: "This is my red sweater, my blue pants," etc.

-- Have your child point to different colors and have him call them by their names. You might ask him: "What is the color of this sign post, of the flower, of the car? What is the color of the traffic light?"

-- Have your child look for different shapes in his immediate surrounding. For instance: The window is rectangular, the plate is round...

-- If your child wears light and gaudy clothing, he will be noticed better and more quickly by others.

- Step No. 5:

-- Act consistently. Wrong behavior on your part might confuse your child. Once the child has acquired good habits such as crossing the street at marked pedestrian crossings, do not give him a bad example by jaywalking.

- Step No. 6:

-- Do not practice too much since too many instructions can be perceived as a burden by your child. Do not forget that children cannot concentrate as long as aduits.

Step No. 7:

-- Give your child more and more responsibility because he must learn to make the right decisions. Ask him questions such as: "What do you think? Shall we cross the street now? Or should we wait?" Af.ter 
several weeks of training, give your child more responsibility by allowing him to act as the leader. Let your child go by himself for a short while but make sure that you are always nearby. Praise him if he deserves recognition. This is especially important.

- Step No. 8:

-- Do not frighten your child. Do not scare him by talking to him about traffic accidents. Conversations of this sort do not make him more cautious but will only frighten him more.

- $\quad$ Step No. 9:

-- Teach your child how to judge distances and speeds. He must know whether or not he has sufficient time to cross the street.

- Step No. 10:

-- Train your child to communicate with other traffic participants. In spite of the green light and pedestrian crossing, your child should first establish visual contact with cars, etc., coming from the left or the right. 
FEDERAL REPUBLIC OF GERMANY

Name of program: Verkehrstraining mit Kindern. Fur Eltern (Traffic Education of Children - A Manual for Parents)

1. Sponsor: Innenministerium Baden - Wurttemberg (one of the states in Federal Republic of Germanyl.

2. Date: April 1979.

3. Kind of safety taught: Pedestrian.

4. Age of children taught: two to seven.

5. Matertals reviewed: We have a copy of the booklet (in German) and a brief (English) summary of its contents.

6. Format:

This is a 43-page booklet fully illustrated with color photographs, colorful comics and diagrams, and carefully designed so that the layout is attractive and easy to read. There is not much writing per page. In addition to the introduction, there are four chapters. Each of these sections has its own bright color around the edge of its pages. The photographs show urban and surburban traffic environments, parents, and children modeling correct pedestrian behavior, and parents instructing their children in pedestrian safety. The comics appear to show children engaged in inappropriate pedestrian behaviors. All in all, the book let looks inviting and attractive.

7. Information presented:

- Why children become traffic victims

- Why parents should train their children to understand and observe traffic rules

- Why parents should first test themselves so as to find out whether they are capable of giving their children good examples in traffic behavior

- A test parents should also give to their children so as to find out their children's reaction in given situations

- Three golden rules for parents:

1. First show your child what to do.

2. Practice together with your child.

3. Show your satisfaction with your child's success by praising him. 
FEDERAL REPUBLIC OF GERMANY

Name of program: Children's Traffic Club.

1. Sponsor: Jugendwerk der Deutschen Shell.

2. Date: Not given.

3. Program participants: Parents and children.

4. Age of children in the program: three through six.

5. Kind of safety taught:

- Pedestrian

- Car Passenger.

6. Club operations:

- The club is essentially a correspondence course. Each year for four years, parents must pay 31.30 D.M. (roughly $\$ 17$ in our currency but considered by the Club to be a minimal cost in Germany) for their children's membership in the club. We are not sure how parents are initially contacted by the club, but we assume that Deutchen Shell obtains the names and addresses of children who are about to become three-years-old from local registries and sends them a club brochure and application card. To enter a child in the club, parents merely fill out the application card and send it and the yearly payment back to the club. The children receive the materials described be low every six months. After children have completed training, they complete and send to the club a special puzzle or game (or some other special assignment). In return, the club sends them a gift-a figure to add to their traffic panorama.

7. Materials reviewed:

- Learning packages, one for the three-year-old and one for the three-and-a-half-year-old.

8. Format of the materials:

- Each child is sent:

-- One learning package every six months starting his/her third birthday and ending when he/she is six-and-a-half. (There are eight learning packages in all.)

-- One colorful four-ring binder with pictures of the safety mascot (a kind of animal) guiding children through various traffic situations 
-- Club privilege card

-- Club identification card in which the child inserts his/her photo, name, and address

-- Club spoon

-. Club sign

-- Toy car

-- A gift every six months, as a reward for solving a small lesson/problem in each learning package and sending it back to the club.

Each learning package contains:

-- An introductory letter to parents which describes the characteristics of children their child's age, gives an overview of the lesson themes, and suggests appropriate teaching methods

-- Four or five lessons, each of which contains a letter to parents, a letter to the child, and a picture on the opposite page

-- A three-dimensional traffic panorama with figures of people and vehicles to cut out.

-- A puzzle or game for the child to complete and return to the club.

- The fllustrations in the pictures are colorful drawings of pedestrians and vehicles in urban, suburban, and rural traffic environments. While the drawings are not totally realistic, they present the traffic environment in great detail. Roads, for example, are a purple color with grates on the sides and grey brick sidewalks with white curbs. Color is lavishly applied to houses, trees, cars, and every other part of the environment. With smoke coming out the chimneys of houses and flowers popping up in gardens, the illustrations are reminiscent of chidren's drawings. The safety mascot, incidentally, appears in different places throughout. The pedestrians shown in the illustrations model correct pedestrian behaviors, and all look very happy doing so. Adults are shown holding children's hands and looking for cars. All people depicted are wearing up-to-date clothes. Several illustrations have spaces where cut-outs of cars or pedestrians' heads fit. In one, for example, children insert cars going in the correct direction on the street. The heads are inserted so that the pedestrians can look in one direction for approaching vehicles. 
A second type of lllustration in the learning package shows several distinct pictures of efther vehicles or places to play (yards, playgrounds, etc.). The pages with moving vehicles show children on toy vehicles, on top of grey bricks (symbol for sidewalks) and the adult vehicles on purple (symbol for roads). The third type of illustration given is the traffic panorama, a brightly colored representation of a distinct traffic environment which folds out and up so that streets and sidewalks are on the flat and houses $r$ ise above at a 90 degree angle. Children are given figures of pedestrians and vehicles to use when they play with the panorama. The environments are as child-like, colorful, and detalled as those in the first type of pictures.

9. Concepts taught to three-year-olds:

- Meaning and use of sidewalks

- Meaning and use of streets

- Where toy vehicles belong and why

- Where to walk when there's no. sidewalk

- Safe places to play.

10. Concepts taught to three-and-a-half-year-olds:

- The directions in which vehicles travel on the roadway

- Different movements of vehicles (e.g., stop, drive on, brake, park)

- What to do when someone calls to you from the across the street.

11. Pedestrian instructions given to three- and three-and-a-half-yearolds:

- Walk on the sidewalk, not in the street.

- Ride toy vehicles on the sidewalk, not in the street.

- On streets without sidewalks, walk on the shoulder of the road on the left side so that you are facing traffic.

- To cross the street, first stop at the edge of the road, look in both directions, and when no cars can be seen, walk across the street--but look for traffic the entire time.

- When someone calls to you from the other side of the street, you should stand on the sidewalk, look in both directions for cars, and then if no cars are coming, cross to meet the person.

- Children riding in a car should ride in the back seat and wear a seat belt. 
12. Teaching methods used:

- Book with illustrations read repeatedly to the child

- Repeated on-street training in real traffic (e.g., walks to observe the traffic environment and practice correct pedestrian behaviors)

- Questions which encourage the child to point out and discuss what he/she knows

- Use of concrete objects or places in the traffic environment to orient children so. that they are able to look left and right

- Drawings (by the child)

- Three-dimensional traffic panoramas (simulated traffic environments) with figures of pedestrians and cars so that the child can reenact real traffic and book lessons

- Puzzle of program mascot and another animal to fit together

- Parent modeling correct pedestrian behaviors

- Providing answers to children's spontaneous questions

- Consistency in road training--having the child practice crossing the road at the same spot.

13. Measurement of children's knowledge and behavior:

- None suggested. 


\section{F INLAND}

Name of program: Pim Pam Pelista Pois

1. Sponsors: Lilkenneturva and the Finnish Broadcasting Company.

2. Oate: 1977 (publication date of Kijpeli).

3. Age of children in the program: four-and five-year-olds.

4. Kind of safety taught: Pedestrian.

5. Materials reviewed: Kijpell (a traffic book for both children and parents sponsored by the Ministry of Traffic and The Traffic Safety Club which is sponsored by Likenneturva).

6. Use of the television series and the book:

- Pim Pam Pelista Pois is a television series consisting of three shows, each of which lasts 25 minutes.

- Before the program is broadcast, Liikenneturva sends Kijpeli, a book let which complements the television series, to parents and their children. Lilkenneturva and the Ministry of. Communications are responsible for distributing the booklets. Li ikenneturva obtains the addresses of parents with young children through the population registry.

7. Format of Kifpeli:

- Kipeli is a 32-page booklet with colorful child-like cartoon pictures, black and white drawings, photographs of the real traffic environment, game-like activities for children, and text. Its cover is sky-blue with cartoon-like colorful scenes of children in the playground, in the country, on a city street and in a city yard: The cover reminds parents that the book is for both parents and children and adds a personal touch by supplying a space where a child can write his/her name. Inside the cover, there are many types of pictures of the traffic environment. Many wide-angle photograptis present the traffic environment as a whole. Sidewalks, signs, cars parked on the side of the road--all are presented in context. Several pictures, particulariy cartoon-type drawings, flustrate specific points being made in the text. For example, two small cartoons show how difficult it is for drivers to see children behind cars and how hard it is for children to see cars. Another double cartoon shows the child's field of vision as contrasted with the aduit's field of vision. The pictures also show several situations which lead to traffic accidents for the four- and five-year-old, e.g.,. dashing into the roadway, playing on the curb, running after a ball in the street. The illustrations present model pedestrian behaviors as well. children of both sexes are shown, and gipls are shown breaking 
pedestrian rules along with boys (i.e., there is no double standard). All in all, the book is very colorful, busy and exciting. Although the basic format consists of a section supplying information for the parent followed by an activity for parents and children to do together, there is no repeated and expected pattern in the format. Thus, the booklet is full of surprises for the child.

- The preface 11 lustrates and discusses how the environment has changed and how these changes have affected young children. Accident statistics are cited.

- The introduction states the purpose of the book and suggests how parents should use the book with their children.

- The next sections discuss:

- The child's need to play and explore the environment--the child's limitations in coping with traffic--the child's need for protection in traffic--the emotional, social, physical, and intellectual characteristics of the four-and five-year-old child

-- General methods for teaching children correct pedestrian skills and attitudes

-- Specific instructions to use when teaching a child to cross a road, take a bus, or ride in the car.

- The last section of the book let focuses on improving the environment so that young children will have places to play away from trafic and so that existing places will be made safer. Safety criteria for assessing an environment are given. Suggestions for improving the safety of the home and yard are suggested and the names of local and county organizations in different parts of Finland are listed, so that the parent will know whom to contact to request that changes in the environment be made. A story about young city children who discover wildife at their grandparents' place in the country is also included in this section.

- Throughout the booklet, activities for young children are included. Some activities are directly related to traffic. For example, the child is asked to identify various parts of the traffic environment in a colorful child-like scene of an intersection, playground, yard, streets, and sidewalks. On another page children are asked to draw a picture of the kind of play area they would like to have. They are asked to select and color the pictures which show safe play areas, to draw a line through a maze, to draw the last picture in a sequence of pictures about crossing the street, to color a traffic light and sign, to interpret a series of pictures about a bus trip, and to connect dots. A few activities are focused more on perceptual skills such as noticing differences between two similar clowns. 
8. Pedestrian concepts in Kifpeli taught to children in the following order:

- What a sidewalk, pedestrian crossing, gate, yard, and playground look like--i.e., parts of the traffic environment

- Things that belong in a playground and trings that don't belong (such as trucks)

- Safe and unsafe play areas

- Idea that certain things belong in certain parts of the environment (e:g., pedestrians pushing baby carriages belong on sidewalks, not in fields)

- Procedure for crossing the street

- What a trafific light and pedestrian crossing sign looks like

- How a child should walk with an adult (ile., which side) when walking near the roadway

- Where to walk near a highway

- Why it!s dangerous to play and run on the sidewalk and highway

- Where to cross a road

- How to use a pedestrian crossing.

- How to cross at an intersection

- Where to stand to wait for a bus

- How to disembark from a bus and enter traffic

- Why speed limits exist.

9. Pedestrian instructions taught to children in Kijpeli:

- Play in the yard or at the playground, not in the street or next to the street.

- Cross the street at a pedestrian crosswalk. When you come to a crosswalk, stop. look both.ways (and behind at junctions) to see if any cars are coming, and then cross if it is safe.

- When a: child is walking with a grown-up on the sidewalk or highway, the child should hold hands with the adult and walk on the inside so that he/she is farther away from traffic.

- Walk on the left side of the highway.

- Don't run or play on the sidewalk or highway. 
- Walk--don't run--across the road.

- Wait for the bus at a safe distance away from the road.

- When you get off a bus, wait until the bus has left before you cross the road.

10. Teaching methods used:

- Parents modeling correct pedestrian behavior

- Parents answering children's questions about traffic

- Parents talking to the child about how to behave in traffic and why, when they are out in real traffic situations together

- Parents and children talking about the pictures in Kipeli and doing the representational activities le.g., maze, drawing together.)

- Parents and children viewing the television programs together and discussing them

- Parents creating a safe environment where children can play and safe environments so that children can walk places safely by themselves.

11. Measurement of children's knowledge and behavior:

- No assessment instruments are suggested.

12. Evaluation of this pedestrian safety program:

- Surveys are being conducted to find out the extent to which the program is being used, how much information participants have gained from the program, how this knowledge has affected their attitudes toward their children's behavior, how such knowledge has affected their methods of teaching their children, and whether they feel that the program has met their needs. 
FINLAND

Name of program: Noppa:

1. Sponsors:

- Liikenneturva (Central Organization for Traffic Safety)

- Yleisradio (The Finnish Broadcasting Company)

2. Date: Since Autumn 1976, traffic instruction has been incorporated into the NOPPA series.

3. Age of children in the program five-to eight-year-olds.

4. Kind of safety taught: Pedestrian.

5. Materials reviewed:

- Ekaluokkalaisen Lijkennevinko (A Traffic Book for a First Graderl.

6. Use of the television series and book:

- Noppa is a television series. It consists of seven television programs, each of which is 25 minutes long. Each program is broadcast in the afternoon and repeated later in the evening.

- Children in the first grade receive a booklet on road safety (Ekaluokka laisen Lilkennevinko) to be read in conjunction with the Noppa programs. The booklet, written for both children and parents, contains instruction similar to that in the TV programs and information for parents about children's capabilities in traffic and the best ways to teach them traffic safety.

7. Format of Ekaluokkalaisen Liikennevinko (A Traffic Book for a first Grader ):

- This small 12-page booklet has a sky-blue colored cover with a photograph of two children standing next to the roadway. Almost every page in the booklet has one or two beautiful photographs. Overall the booklet is very inviting and attractive.

- The introduction is a letter to parents. In a few sentences, it states children's needs to explore the environment and their need for traffic safety education. It also instructs parents first to discuss the safety principles shown in each picture of the book. with their children, then to practice the lesson outside in real traffic, and finally to check off the lessons they have completed on the record-keeping form on the bottom of that page. 
- Each page of the pages that follow contains:

-- The concept to be taught at the top of the page

-- One or two photographs depicting the correct behavior

- Instructions describing the correct behavior and explaining the reason for such behavior

-- Occasional mention of the kinds of accidents children are liable to have if incorrect behavior is used and/or information about children's typical behavior in such situations

-- Instructions to follow when teaching the child outdoors

-- Directions for activities in the book, such as drawing pictures of traffic situations in blank spaces in the book or identifying correct and incorrect behaviors shown in two pictures.

In short, the pictures on the pages are intended for parent and child, while the script is intended to inform the parent and provide instructions to give to the child.

- The pictures in the book are of great importance. Many types of traffic environments are displayed--inner city areas with no grass or trees, city areas with apartment buildings, houses and grass, and rural areas. Two nighttime scenes on a snowy rural road and many daytime scenes are shown. The same two children, a boy and a girl, appear in most of the pictures. Scenes are photographed so that as much of the whole environmental context that can be shown is shown. Thus, signs are shown in their full context. The sequence of looking in both directions and behind is shown in three pictures of the same children in the same place looking in three separate directions.

8. Concepts in Ekaluokkalaisen Liikenneturva taught in the followingorder:

- How to walk and where to walk when coming from the house or yard to the sidewalk or highway

- Use of the sidewalk and how to behave when using it; safest way to school (not discussed, just mentioned)

- Where to walk when there's no sidewalk next to the highway or when there's a pedestrian path next to the highway

- Meaning and proper use of a pedestrian crossing

- The reason to stop and when to stop before crossing the street

- How to scan for vehicles before crossing the street 
- How to cross the highway with and without an overpass or subway

- The dangers of walking at dusk and at night and the need for reflectors

- How to interpret traffic lights and cross the street with a traffic light.

9. Pedestrian instructions given:

- When coming from the yard or entrance, walk rather than rush and continue walking along the sidewalk (if one exists) or along the edge of the road.

- Walk on the sidewalk as far away from cars as possible. Don't run or play on the sidewalk.

- When there's no sidewalk next to the highway, walk on the left side of the highway facing oncoming trafic. Walk single file. Don't run or play on the highway. When a pedestrian path is avallable near the highway, use it. observe and obey pedestrian and bicycle road signs.

- Cross the road in a pedestrian crossing. Don't cross the street from between parked car.s.

- Stop before crossing the street and look to see if any vehicles are coming.

- Look for vehicles coming from both directions before you cross a street. Look in both directions and behind for approaching vehicles when crossing at an intersection. When there are no cars nearby or when the cars have stopped, you can start to cross the street.

- Use overpasses and subways on the highway whenever they exist. If none exist, find a place on the highway which allows you to see in both directions and stop. If you see no cars, you can walk quickly across the road.

- When walking at twilight or at night, use a reflector. It's a good idea to sew reflectors on your coat:

- At a traffic light when the green light is on you, you can cross the street after you have looked in both directions and behind to see that the cars have stopped. Do not cross the street on the red light.

10. Teaching methods used in Noppa. and Ekaluokkalaisen Lilkennevihko:

- Television

- Songs (taught on television) 
- Televised role-play

- Individualized on-street training in the real traffic environment by parents

- Picture books

- Peer modeling: children watching television see other children who tell and show them how to act in different traffic situations (in real traffic).

11. Measurement of children's knowledge and behavior:

- No formal instruments are suggested, but parents are encouraged to observe their children's behavior closely.

12. Evaluation of this traffic safety program:

- The University of Jyvaskyla has conducted an ongoing study of this program, and as a result, the program's content and teaching methods have been updated and modified. 
Name of program: Traffic Education Programme for Preschool Aged Children and Children Starting School

1. Developers: Tapio Nummenmaa

Kaar in Ruuhilehto

Matti Syvanen

(Department of Psychology, University of Tamperel.

2. Sponsors: Finnish National Board of Education and Likenneturva (Central Organization for Traffic Safety).

3. Date:

- Program development began in 1972.

- The report on the program was issued in 1975.

- This program was part of a study to prepare a traffic instruction program for preschool children and to test whether such a program could be taught by both teachers and parents. Information was collected in order to determine the types. of arrangements needed for parents and teachers to cooperate efficiently and the kinds of guidance that each needed to teach in an effective manner. It is our understanding that research from this study contributed to the development of the NOPPA program.

4. Age of children in the program: six-year-olds.

5. Kind of safety taught: Pedestrian.

6. Material reviewed: Traffic Education Programe For Preschool Children and Children Starting School (report).

7. Program format:

- Teachers and parents were the primary instructors in this program. Af.ter they received training in the curriculum, teachers and parents set up a cooperative teaching arrangement whereby teachers instructed children in the classroom by showing them a series of slides, and parents later. followed up this training by giving children instructions in real traffic. Thus, children were introduced to a concept and set of behaviors through a series of slides, and the next day practiced the behaviors advocated in the slides in real traffic situations under their parents.' supervision.

- The slides showed real traffic situations. There were two types of slides, those which. showed correct pedestrian behaviors and those which presented incorrect pedestrtan behaviors. 
- Parents were encouraged to train their children at familiar traffic locations.

- After parents completed a training session, they recorded their observations in a diary.

- Parents and teachers were given the following to aid them in carrying out their part of the training:

-- Schedule of classroom training sessions and general instructions for coordinating home and school training

-- Training content

-- Information on developmental capabilities of preschool (meaning six-year-old) children

-- Suggestions for appropriate training sites.

8. Concepts taught and pedestrian instructions given:

- Essentially, the same as those in Noppa. The set of instructions which teachers were given for presenting the silide series were slightly more detailed and wordy than the instructions in A Traffic Book for A. First Grader. Another difference is that incorrect behaviors were shown and discussed at length in the slide series. Not having seen the Noppa television series, we are unable to compare that film series with the slide series developed by Nummenmaa, Ruuhilehto and Syvanen.

9. Teaching methods used:

- Slides of appropriate and inappropriate pedestrian behaviors used by real pedestrians in real traffic

- Classroom training with representational materials, followed by on-street training as soon after the classroom training as possible

- Parent and teacher cooperation

- Training children on familiar traffic routes

- Training only the correct pedestrian action, but pointing out examples of incorrect pedestrian behavior

- Using simple language and unambiguous concepts

- Replacing "right" and "left" by other words ("one side" and "another side") 
- Asking questions of the child to find out his/her level of understanding of the concepts or skills presented--e.g., asking the child to evaluate a traffic situation and decide what the appropriate pedestrian behavior should be

- Parents modeling correct pedestrian behaviors for the child

- Explaining why certain pedestrian actions are appropriate or inappropriate.

10. Measurement of children's knowledge and behavfor:

- Parents kept a diary of their observations of their children's behavior in traffic.

11. Evaluation of this traffic safety program:

- Parents were surveyed to find out their opinions about the training book they received, the most relevant and most difficult pedestrian instructions taught to their children; any changes they!d observed in their children's behavior, and any restrictions they'd imposed on their chilidren's mobility. in the neighborhood: or any restrictions they. planned to impose in the future. Most parents were reported to be satisfied with the clear and thorough way concepts were presented in the book let. Some parents suggested incorporating clear photos into the book. Noting how the program affected their children's beinavior, parents reported that their children showed the most change in the pedestrian crossing and its use and in looking aside and behind. The third greatest change was that children began to stop before crossing the street. Areas in which the least changes were noticed were walking on the sidewalk and use of traffic lights. Parents accounted for the lack of change by stating that children had known those concepts well even before the training took place. As a result of the program, many parents said that they changed their own behavior by allowing children to walk. independently on routes which involved no street crossing. However, most parents also defined where children could and could not go more closely. 
Name of Program: Children's Traffic Safety Clubs

1. Sponsor: Local governments.

2. Date: The first clubs, the "Antelope Club" in the Yamagata Prefecture and the "Elephant Club" in the Gifu Prefecture, were established in 1971. The Office for Traffic Safety Measures of the Prime Minister's Secretariat advocated nationwide establishment of the clubs in 1972. As of September 30, 1977, there existed 15,000 clubs in 47 prefectures (The White Paper on Transportation Safety, 19791.

3. Age of children in the clubs: three to six years.

4. Kind of safety taught: Road Safety.

5. Club operations:

- The clubs are organized through voluntary efforts of local residents, chiefly mothers. Club membership generally ranges between 40 to 100 members, including children and their guardians. Club meetings are held once a month twelve times during the year. Most club meetings last for the duration of one hour.

- The clubs are financed by local governments through local annual taxes.

6. Materials reviewed: A translation of the booklet for children was reviewed. Of tenuntranslated materials were reviewed for format, illustrations, and techniques.

7. Concepts taught:

- Many of the materials and club procedures used in the Children's Traffic Clubs are based upon those from the Tufty Club from the United Kingdom.

N.B. Sources of the above information:

- The White Paper on Transportation Safety (1979)

- Hoshi, T. Educating Young Children for Traffic Safety. The Wheel Extended. Winter 1976, $6,3,1-22$. 
Additional Pedestrian: Safety Program Materials

- 14" $\times 10^{\prime \prime}$ posters with very colorful pictures of young children in various situations that require safety precautions-at the rallroad track, swimming pool, at the market, on the playground, on the way to school, on streets. Many lllustrations clearly show children who are not observing traffic rules. There are seven pictures per page. Most fllustrations (drawings) are quite realistic. There are also a few actual photographs of real traffic situations on the posters. Two of the posters appear to be traffic safety games. On the back side of each poster is a rather lengthy text.

-- A 60-page book let with a cover picture of mother holding a child's hand while they're walking on the sidewalk. Illustrations inside the book are less realistic and detafled than those on the posters. It looks as if the book might contain directions for parents who are teaching traffic rules to their children.

-- A sturdy 26-page book for children: The cover has three crayon people--red, yellow, and blue--who have drawn Japanese characters. The first sections of the book appear to be teaching colors. Midway through there are two fairly realistic drawings of intersections in cities. Both drawings show traffic lights. One of the last pages also shows a traffic light. The last four pages have photos of children and adults in real traffic situations and a short text accompanying. each photo.

-- A 116-page book of what appear to be games and directions, as well as songs to be taught to children. 


\section{LUXEMBURG}

Name of Program: The Billy Club

1. Developers: The German Road Safety Association, with approval given by the Family Helfare Administration.

2. Sponsor: Luxemburg Road Safety Council.

3. Date: Approximately 1979.

4. Age of children in the program: Ages three to seven.

5. Kind of safety taught: Pedestrian.

6. Materials reviewed: None

7. Program format:

- Every six months children are sent a $k i t$. (There are eight kits in tota1.) Each kit contains a text, puzzle, present, route to school map, road situation to figure out, club card, and test.

8. Club operations:

- The Banque Internationale in Luxemburg finances four-fifths of the costs, equivalent to $100 \mathrm{LF}$ per child.

N.B. The above information was extracted from: Gallienne, G. and Pansard, C. La Prevention Routiere Internationale help ze 'n handje!, 4 th quarter $1979, \underline{6}, 1-20$. 


\section{THE NETHERLANDS}

Name of brochure: "Help ze 'n hanje"

1. Issued by: Veilig Verkeer Neder Iand.

2. Date: 1977.

3. Target audience: Parents of young children, probably.

4. Kind of safety taught: Pedestrian.

5. Material reviewed: Brochure was reviewed but was not translated into English.

6. Format:

- This six-page brochure has drawings of parents and children walking and riding their bicycles in traffic. The drawings are in pen and ink. They are quite realistic and the scenes depicted appear to be recent. There are six boxes with suggestions for parents within. 
THE NETHERLANDS

Name of brochure: "Kijk Samen Uit!"

1. Issued by: Veilig Verkeer Neder land.

2. Date: 1978.

3. Target audience: Parents of young children.

4. Kind of safety taught:

- Pedestrian (primarily)

- Bicycle.

5. Material reviewed: Brochure was reviewed, but was not translated into English.

6. Format:

- This six-sided brochure contains eight black and white photographs of correct pedestrian behavior. Traffic environments shown appear to be urban, suburban, and rural. Near each picture is a text which contains the lesson. One sees a parent holding her child by the hand and bending down to the child's eye view to explain the workings of the traffic light signal. 
NORWAY

Name of program: Children's Traffic Safety Club

1. Sponsor: Trygg Trafikk.

2. Date: Since 1966. (Norway originated the concept of the Children's Traffic Club.)

3. Age of children in the program: three to seven years.

4. Kind of safety taught to children: Pedestrian.

5. Club operations:

- The club is operated by Trygg Trafikk. It is essentially a correspondence course. Trygg Trafikk buys children's addresses from the Statistical Central office and sends every child in Norway both a pamphlet ("This is the Children's Traffic Club") and a payment card shortly before the child's third birthday. Approximately 40 percent of the children in Norway become club members. At present parents must pay a fee of $N . \mathrm{Kr}, 30$ for their children's membership in the club for four years. Every six months club members and their parents receive materials directly from Trygg Trafikk. Included in the teaching materials is a special assignment or test which the child completes and returns to the club. After club headquarters has received the completed test, it sends the child a cut-out figure to put on the child's colorful traffic panorama. The panorama, in a sense, is a reminder to the child of all the assignments and training the child has completed. The club's materials are described in greater detafl below.

6. Materials reviewed:

- Booklets for the three, three-and-a-half, four, four-and-a-haif, and five-year-old.

7. Format of the Children's Traffic Club materials:

- Initially the child receives a red loose-leaf binder, worksheets which fit into the binder, a record, and a large colorful traffic panorama (with street, sidewalk, and buildings, etc., but no people, cars, or safety features). The panorama is called "My Traffic Tests." Every six months the child receives a new set of worksheets and another record. The worksheets contain pictures, text, puzzles (cut and paste assignments), games, and a special test which the child completes and returns to the club. 
- Parents receive book lets supplying information on:

-- How to drive on slippery roads

-- Children and bicyciing

-- Traffic, driving and alcohol

-- How to drive in the dark

-- First-aid

-- Home, accident, car and life insurance

-- Cars passing each other on the road

-- How to make the environment safe for children

-- How to make playgrounds and children's play safer

-- Rules and regulations for drivers and pedestrians.

- Parents also receive pamphlets about the characteristics of three, four, five, and seven-year-old children and general suggestions for teaching them pedestrian safety every time the child has a birthday.

- Each of the children's assignments contain one model picture that fllustrates the concepts to be learned and several other sheets which fold out and become panoramas. These panoramas contain dotted lines which indicate where the figures (to be cut out of a separate sheet) are to be placed. For example, one of the three-year-old's panoramas shows a street scene with a store and house, sidewalk with dotted lines indicating where pedestrians should go, and a street with dotted lines indicating where the car should be placed. The drawings of the panoramas and figures are colorful and bright. While the traffic environments depicted are somewhat stylized and childilike, they are very recognizable. Traffic safety features such as curbs, streets and pedestrian crosswalks, are clearly shown. For example, streets are grey, while curbs and sidewalks each have slightly different colors. Most of the scenes are daytime scenes, except, of course, for the unit on walking in the dark which really shows darkness. Rural, suburban, and urban traffic environments are depicted, with perhaps more rural and suburban environments shown. The environments are illustrated with the freshness of a child, particularly in the snow scenes which show mounds of snow and youngsters in bright-colored snowsuits on their sleds.

8. Concepts taught to three-year-olds:

- Safe places to play

- Use of sidewalks/use of streets

- Vehicles that move on the roadway

- How a child and adult should walk together on the sidewalk. 
9. Concepts taught to the three-and-a-half-year-old:

- Where to walk when there's no sidewalk and what to do if a vehicle approaches

- How a child and adult should walk together when there's no sidewalk.

10. Concepts taught to the four-year-old or four-and-a-half-year-old:

- How to cross the street which has a sidewalk

- How to use a pedestrian crossing

- How to cross a road which has no sidewalk.

11. Concepts taught to a four-and-a-half-year-old or four-year-old:

- Where to sled

- Difficulties of walking in snow, bad weather, and darkness.

12. Concepts taught to a five-year-old:

- Dangers of walking in the dark and ways to minimize the danger

- Where to put reflective tape on clothing.

13. Pedestrian instructions given to children:

- Play in the playground.

- Walk on the sidewalk.

- A child walking on the sidewalk should hold his/her parent's hand and walk on the inside so that the parent is between the child and the street.

- If there is no sidewalk, walk as far to the left as possible on the shoulder of the road.

- Stop before you cross the street or road, wait, listen for cars, and look left-right-left for vehicles before crossing. Cross when no vehicles are coming.

- Use a pedestrian crossing if avatlable.

- When there is no pedestrian crossing, cross at corners or stretches of road where you can see all parts of the road. 
- Walk straight across the street.

- When you're walking next to the highway and a vehicle approaches, stop while the vehicle passes.

- Sled on a place that has a fence between the hill and the roadway.

- Wear reflective tape on clothing when walking in the dark.

14. Teaching methods/materials used:

- Records with songs and instructions on correct pedestrian behavior

- Discussion between parent and child about the club materials' illustrations

- Puzzles which parents help children do (one cuts out pictures and places them in the broken lines provided on fllustrations)

- On-street traffic training of children by parents (short training sessions repeated of ten)

- Discussions about the traffic environment and correct pedestrian behaviors while in the real traffic environment

- Use of simple words and expressions when talking to children about traffic (e.g., substituting other words for "right" and "left")

- Spot (informal) tests of children's abilities to perform correctly in various pedestrian situations

- Reminders to the child that the traffic situation being experienced is the same as that which is depicted in the club materials

- Use of signals such as squeezing the child's hand or telling him/her "now we stop and look to both sides," to alert the child to stop and look before crossing the street

- Experiment seeing how well reflective tape can be seen in a darkened room when the flashlight shines on it--also comparing the effectiveness of the reflective tape versus that of white clothes

- Sewing reflective tape to the child's clothing and allowing the child to take part in some of the sewing. 
15. Measurement of children's knowledge and behavior:

- Every six months (or after training is completed), each child is given a special assignment to do. It is returned to the club and in return the child receives a sticker. The special assignment for the three-year-old is a puzzle. The assignment for the four-year-old requires the child to show the parent where and how to cross the street correctly three separate times.

16. Evaluation of this pedestrian safety program:

- An analysis of the "Children's Traffic Club" was conducted by Per Schioldberg, Ph.0., the Institute of Psychology, University of 0s 10, (ISBN 82-990349-0-6, 1974, 134 pp).

- The analysis of the effect of the Children's Traffic Club on parents was based on postal interviews of members and non-members; interviews focused on parents' views on children and traffic and the kind of training experiences which member-parents had with the CTC.

- More than 400 five- to six-year-old children and 600 parents participated in the study.

- Results:

"For the children, the results showed that members had a significantly higher proficiency level than non-members, but only among the girls $(t=2.51$, df $=38, p 0.02)$. The difference was 20 percent, being more pronounced in spatial orientation and road sign cognition than in situation and concept understanding. This result remained unchanged with regard to reproduction of traffic knowledge as compared to recognition of traffic elements in a model situation.

Concerning the correctness of traffic behavior displayed by the children when alone or together with other children, no significant difference was observed between members and non-members when the material was analyzed as a whole. However, when comparing children walking alone with children walking with others, the members showed a significantly higher level of correctness (45 percent) than did children walking with others, irrespective of the latter being members or not $(t=2.20$, df $=108$, $p$ $0.05)$. For non-members a lone, the corresponding difference was considerably smaller ( 15 percent) and insignificant. Unexpectedly, this finding proved more pronounced among boys than gir is. 
The analysis of the accident risk of members versus non-members included all children born 1966 and 1967, involved in road accidents in 1972 as active parties. When separating this accident group into members and non-members in relation to their corresponding populations, risk values per 10,000 chilren are computable, as illustrated in Figure 2. Thus, the accident risk proved 20 percent smaller for members compared to non-members for Norway as a whole (14.2 vs. 17.8 per 10,000 children), for 0s lo as much as 40 percent smaller $(31.4 \text { vs. } 55.7)^{*}$. This difference in risk level also varies with sex; the reduction in accident risk for girls and boys was, for Norway, 40 percent and 10 percent, respectively; for 0510,25 percent and 50 percent. In general, the accident risk proved twice as large for boys than for girls, regardless of membership. This unfor tunate result, however, is in accordance with international statistics.

The analysis also proved that the lower accident risk obtained for the members could not be attributed to a more favorable traffic milieu for this group. The difference in risk level for the two groups ought, therefore, to be considered in light of the results obtained among the parents. Here, member-parents proved to be more restrictive and safety-oriented towards their children than non-member-parents, while both groups were equally insecure of the child's ability in traffic. Ninety-five percent of the member-parents approved of the instrument both as a training guide and a source of information reinforcing their traffic safety attitude.

The results of this study indicate that the interaction between instrument, parents and children benefits the latter in increasing their profictency and behavior level and in decreasing their accident risk. However, the results also showed how vulnerable chtldren still are in traffic, and that traffic training has its limitations."

(Quoted from: Schioldberg, P.: Children in Traffic. ITE Journal, June 1978.) 


\section{SWEDEN}

Name of Program: Children's Rodd Safety Club

1. Sponsor: Nationalforeningen For Trafiksakerhetens

Framjande(NTF) (National Association for the

Prevention of Road Accidents).

2. Date: Since 1969.

3. Age of children in the program: three to seven years.

4. Kind of safety taught: Pedestrian.

5. Club operations: Sweden's Children's Road Safety Club is a correspondence course. Every six months families are sent instructional materials.

6. Materials reviewed: All materials for ages three through five-and-a-half.

7. Format of the materials:

- There are nine booklets in the entire club series (for ages three, three-and-a-half, four, 4 and-a-half, five, five-and-a-half, six, six-and-a-half). The book lets are sent in bright colored envelopes to children and their parents every six months and are put into a red four-ring binder. Thus, as the child gets older, his/her book of units gets thicker.

- Each booklet contains the following:

-- Information for parents on the characteristics of children their child's age. This information is accompanied by black and white drawings of children of that age.

-- One page (at least) which depicts a traffic environment with model pedestrian behavior shown.

- Activity pages for parents and children to do together. These pages are brightly colored lllustrations of parts of the traffic environment with blank spaces and/or white shapes where punch-out glued figures can be pasted. Directions are also provided for doing the cut and paste learning activities.

-- Text for parents. The text lists questions for parents to ask children when looking at the model page and activity pages and when training the child in traffic. Answers to the questions are also provided, along with background information for the parent on children's typical behavior in traffic and tips for on-street teaching of pedestrian safety. 
- The illustrations in the booklets are colorful, realistic drawings. Every inch of the page has color on it. People are wearing bright colored clothes and buildings, cars, sky, and vegetation add even more color. The road, when not snowy, is a lways a solid grey brown. A great deal of detall is shown, thereby making single parts of the traffic environment such as signs, crosswalks, curbs, etc., very easy to recognize. Urban, suburban and rural traffic environments, night and daytime scenes, snowy, sunny, and rainy weather are all depicted. Pedestrians of dffferent ages modeling many correct pedestrian behaviors, as well as different kinds of vehicles, are shown. The use of color and detall make the materials attractive and inviting.

8. Concepts taught to three-year-olds:

- Meaning and use of sidewalks

- Meaning and use of streets (means both sidewalk and roadway)

- Meaning and use of roadway.

9. Concepts taught to three-and-a-half-year-olds:

- Meaning and use of crosswalks

- How to stop, cross the street

- Meaning of pedestrian crossing signs (if the child begins asking about them)

- How to judge when it's safe to cross the street

- Dangers of all kinds of moving vehicles.

10. Concepts taught to four-year-olds:

- Where and how to walk along country roads with no sidewalks

- What to do when walking next to a country road and a vehicle approaches

- Different kinds of vehicles found in the country.

11. Concepts taught to four-and-a-half-year-olds:

- Where and how to walk on snowy country roads with no sidewalks

- Where to sled and ski and play in winter

- Meaning of pedestrtan signs. 
12. Concepts taught to five-year-olds:

- Meaning of traffic light colors and appropriate vehtcle and pedestrian behaviors

- Meaning of pedestrian walk light signals and appropriate pedestrian behaviors.

13. Concepts taught to five-and-a-half-year-olds:

- Dangers of walking in the dark

- Use of reflectors

- Where to put reflectors on clothing.

14. Concepts taught to six-year-olds:

- Uses of subways, overpasses, and pedestrian paths

- Role of police officers

- Meaning of the pedestrian sign

- Where to walk on bridges and next to highways.

15. Concepts taught to six-and-a-half-year-olds:

- Dangers of trains

- What railroad crossings look like

- Meaning of safety features used at railroad crossings and appropriate pedestrian behaviors

- Where to wait to board the bus on a country road.

16. Concepts taught to seven-year-olds:

- Components and workings of the whole traffic system

- Where and how to walk beside a highway

- Review of uses of reflective tape for walking in the dark

- How to interpret vehicular turn signals

- How to cross at three- and four-way intersections

- How to cross the street at mid-block when parked cars are blocking visibility. 
17. Pedestrian instructions given to three to five-and-a-half-year-olds directions and listen for cars. If no cars are coming, walk across the street between the crosswalk lines.

- Walk straight across the road.

- If there is no sidewalk, walk on the left side as far away from the edge of the road as possible.

- Cross at street corners if there is no sidewalk.

- When a vehicle approaches you and you are walking next to the roadway (because there are no sidewalks), walk as far to the edge of the road as possible and stand still until the vehicle has passed.

- Walk single-file when walking on the left shoulder of the road facing traffic.

- Wear reflectors on clothing when walking at night.

- Don't play or talk while crossing the street.

- Look at the top of the "Walk" light to see whether the standing red man or the walking green man light is on. Stop and wait if the red man light is on. If the green man light is on, first look to make sure no cars are coming or that all cars have stopped before crossing.

- When the red or yellow traffic light signals facing you are itt, stand still and wait on the sidewalk. When the green light facing you is 1it, you must first look in all directions for approaching cars and cross when no cars are coming or when they've stopped. 
- When it's raining, look for cars extra carefully.

18. Teaching methods/materials used:

- On-street pedestrian safety training given to the child by his/her parent(s)

- Interpretation of 11 lustrations of pedestrians modeling correct behaviors--letting the child state what comes to mind first before asking directive questions

- Cut and paste puzzle activities which parents and children do together

- Parents modeling correct pedestrian behaviors

- Parent and child discussion about parts of the traffic environment and pedestrian behaviors while walking in traffic, as well as indoors, while doing the club puzzle activities

- Teaching the child one rule at a time

- Frequent repetition of pedestrian instructions during indoor and outdoor traffic instruction

- Use of simple language when talking to the child (e.g., substituting "one way" for "left" and "the other way" for "right")

- Squeezing of the child's hand to alert him/her to stop before crossing the street

- Going to the child and talking to him/her rather than shouting to $\mathrm{him} / \mathrm{her}$ from across the street

- Parents holding the child's hand while in traffic

- Record which repeats pedestrian instructions in songs

- Parents reviewing the material first before discussing it with the child

- Asking questions of the child to determine his/her level of understanding and let him/her demonstrate how to behave

- Test for child--while walking in three different places, the child should show where to walk (either on sidewalk or on left shoulder of road), what to do when a vehicle approaches

- Experiment seeing how well both a reflector and a doll can be seen in a darkened room when a flashlight is shined on them 
- Use of aerial pictures to show traffic environment (starts when children are six-and-a-half).

19. Measurement of children's knowledge and behavior:

- Children are given behavior tests and are observed while fitting together the activity puzzles.

20. Evaluation of this traffic safety program:

- Parents were surveyed by the Children's Traffic Club (BTK) in order to find out how the club was received in the home and how it was used in the family unit. This report, called the "Summary of a Field Survey of the Club's Reception in the Home," states that, in general, the club materials were positively received in the home, that the materials appeared to have positively affected both children's and parents' attitudes towards traffic. The results of the survey also indicated that some segments of the material need to be revised to add clarity and that some additional information should be given. The report suggests undertaking a comparative study of children who have been part of the Children's Traffic Club vis-a-vis those children who have not participated to find out whether an increase in knowledge and reduction of traffic accidents has occurred as a result of the club. 


\section{SWITZERLANO}

Name of Program: A Letter to Children from the B.P.A.

1. Sponsor: Swiss Accident Prevention Bureau (B.P.A.)

2. Date: Starting in 1979.

3. Program participants: Parents and children under six.

4. Kind of safety taught:

- Road

- Playground

- Home.

5. Materials reviewed: Newsletters

6. Program format:

- This letter provides parents with suggestions for teaching traffic, playground, and home safety to children in different age groups.

- The letter is distributed to parents through the B.P.A. community representatives.

N.B. The above information was extracted from: Gallienne, G. and Pansard, C. La Prevention Routiere Internationale--helpze 'n handje!, 4 th quarter, 1979, 8 . 
UNITED KINGDOM

Name of Program: Children \& Traffic (teaching guides)

1. Developers: Road Safety Education Project directed by Ken Jolly and based in the School of Education at the University of Reading.

2. Sponsor: The Transport and Road Research Laboratory.

3. Publisher: Macmillan Education.

4. Date: 1977.

5. Kind of program: School.

6. Age of children in program: five to thirteen years.

7. Kind of safety taught:

- Pedestrian

- Car passenger

- Bicycle.

B. Books in the series:

- 1 On the Pavement (for five to seven year olds)

- 2 The Young Traveller (for eight to ten year olds)

- 3 Preparing for the Road (for eleven to thirteen year olds)

9. Materials reviewed:

- 1 On the Pavement.

10. Format of 0 n the Pavement:

- The preface discusses children's accident rates, justifies the importance of giving children traffic safety education, and outlines goals, objectives, and teaching methods for a traffic safety program for young children. It stresses the need for a small teacher-pupil ratio for on-street traffic training and the importance of parent involvement in such a program.

- The introduction gives a brief overview of the five-to-sevenyear-old's increasing independence but his/her continued need for adult guidance and protection in the traffic environment. The introduction explains that the curriculum 
capitalizes on children's natural curiosity and need to explore, by giving children opportunities to observe, listen to, and ask questions about the real traffic environment they are experiencing. Children's need to create and interpret what they experience is met through a variety of creative experiences such as drawing and role playing.

- The main text is divided into the following sections: the sidewalk and its surroundings; bicycles; types and characteristics of vehicles; road safety personnel; ice-cream trucks and delivery vehicles; and, lastly, crossing roads. A list of books and information about where teaching aids can be obtained follows the text.

- Each section contains: concepts to be presented to the children and suggested teaching methods. Although numerous exciting learning activities were described so that any teacher could use them, it appeared that the developers were more concerned about communicating a basic approach to traffic safety education--in short, giving children opportunities to observe the traffic environment, practice correct behaviors and create, through a variety of mediums, everything they have learned.

- The book is 11lustrated with many color and black and white photographs of children who are experiencing the real traffic environment, and children who are creating charts, pictures, stories, plays, and models in the classroom. Children look absorbed in their activities. It is clear from the pictures that they are developing academic skills at the same time that they learn about traffic environments and develop pedestrian sktils. Drawings and accident graphs are also included.

11. Concepts taught to five to seven year olds in the following order:

- How children travel to school (by foot, by car, etc.)

- Identification and use of sidewalks, pavements, curbs, gutters, roads, driveways, entries, lampposts, zebra crossings, pelican crossings, road signs, traffic lights, traffic islands, and other features of the traffic environment

- Identification and localization of sounds and smells in the traffic environment

- Where to walk

- Which part of the sidewalk to walk on

- How to cross alleys, driveways, and entries 
- Dangers of walking between the road and objects close to the curb (such as lampposts)

- How to walk with other people

- Safe places to play

- Concepts related to bicycle safety

- Dangers of parked vehicles

- Position and directions vehicles move on the road

- Meaning of a honking horn

- Meaning of car turn signals and lights

- Busy roads vs, quieter roads

- Road safety personne1--who they are and what they do

- How to cross the road at controlled crossing places (where road safety personnel are)

- What to do if lost

- What to do if approached by a stranger

- Cautions to take near ice cream vans and delivery vehicles

N.B. The developer's position regarding the appropriate age for independent street crossing is that infants (ages five to seven) should only cross streets when under the close supervision of an adult or responsible older child. However, to help the many children who are crossing streets unsupervised and to provide a transition to the second book in the series, the developers decided to teach the additional concepts listed below.

- Use of bridges, subways, and pedestrian paths

- The Green Cross Code (first taught deleting "near" so that children cross only if no traffic is coming)

- How to cross very busy roads

- How to cross quiet roads.

12. Pedestrian instructions given to five to seven-year olds:

- Walk on the pavement (sidewalk). 
- Walk as far away from the curb and road as possible, facing the traffic that is nearest you.

- Before crossing alleys, driveways, or entries, stop, look all around, and listen for cars or trucks coming in or out.

- Do not walk between objects placed next to the curb and the road.

- Children should hold adults' hands, the handle of the baby carriage or the hands of older children when crossing the streets and walk next to them rather than lagging behind or running ahead.

- Play in safe places such as parks, back gardens, playgrounds.

- Children should not go into the road alone--to cross the road, chase a ball, or see someone on the other side.

- Watch the directions that vehicles are moving and look for any clues, such as turn signais, that will help you learn which way the car is going to move.

- Obey the road safety personnel.

- Children six to seven years of age should be accompanied by an adult or older responsible child when they go to an ice cream van or delivery truck.

N.B. The following are pedestrian instructions which, in the developer's opinions, are more appropriate for older children but ought to be taught to five-seven year olds because so of ten they are crossing streets unsupervised and need the skills below.

- Children should cross very busy roads only when they can use a bridge or subway or a responsible person takes them safely to the other side.

- To cross a quiet road, first find a place where you can see traffic coming from both directions for a long way away (i.e., do not stand at a busy intersection, bend in the road, at places where there are guard rails or near parked vehicles). Then follow all steps in the Green Cross Code.

- When you want to cross the road and one car stops and waves you to cross, do not cross unless you are absolutely sure that no cars are coming from any direction. 
- The Green Cross Code:

" First find a safe place to cross, then stop.

Stand on the pavement near the curb.

Look all around for traffic and ifsten.

If traffic is coming, let it pass.

Look all around again.

When there is no traffic near, walk straight across

the road. Keep looking and listening for traffic

while you cross.

13. Teaching methods/materials used:

- On-street traffic training with recommended ratio of 1 adult to 5 children (teachers are recommended to enlist the help of parents, road safety personne1, older students or other community members in order to achieve such a ratio). The traffic training has two basic aims--to increase children's awareness of the traffic environment and to train children in actual pedestrian behaviors.

- Follow-up activities such as drawing or painting, role-play, making charts, writing stories, making three-dimensional models (from blocks, boxes and cardboard, etc.), discussing observations.

14. Measurement of children's knowledge and behavior:

- No formal assessment tools were suggested. The classroom is full of children's representational works, which may be used as an indication of the children's knowledge. 
UNITED KINGDOH

Name of Program: Traffic Safety Club

1. Sponsor: Transport and Road Research Laboratory (TRRL).

2. Date:

- Currently being developed

- First booklet published 1978 and pilot tested.

3. Age of children in the program: three to five-and-a-half years.

4. Kind of safety taught:

- Pedestrian

- Car passenger

- Whee1 toys (tricycles, etc.).

5. Club operations:

- It is our understanding that this club, like the children's traffic clubs in the Scandinavian countries, will be a correspondence course. Materials will be sent to children every six months from the time they turn three to (and including) the time they turn five-and-a-half. We have not learned any additional facts about anticipated club operations such as how it will be financed, how membership will be determined, etc.

6. Format of materials:

- The booklets currently being developed will contain information for children and parents. Learning activities for on-street training and indoor learning and rationale for such activities will be given to parents. Children will look at the pictures in the booklets and do the learning activities together with their parents. Additional information about the book let format, objectives, concepts, and teaching methods cannot be described further at this time as the club materials are still in the development process. 


\section{UNITEO KINGDOM}

Name of Program: Tufty Club

1. Sponsor: The Royal Society for the Prevention of Accidents (RoSPA).

2. Date: Since 1961.

3. Age of cildren in the program: three to seven or eight years.

4. Kinds of safety taught:

- Pedestrian (primary emphasis)

- Car passenger

- Home

- Playground.

5. Club operations:

- There are two ways that families can become involved in the Tufty club. They can enroll their children as individual members and receive Tufty materials through the mail on a regular basis, or they can affiliate themselves with a group and use Tufty materials both at home and at group meetings. RoSPA highly recommends the group approach.

- The "Tufty Club" was formed in 1961 to teach young children in Great Britain the rules of correct road behavior. Tufty, the squirrel who gave his name to the movement, is the central figure in the materials which illustrate the road safety lessons. Membership was originally intended for "under-fives," but the popularity of the club has grown so that children up to seven or eight years of age are enrolled. Membership rolls are increasing at the rate of approximately 1,000 members a week.

- The national headquarters of the Tufty Club is under the administration of ROSPA. There are regional offices in England, Scotland, Wales, and Northern Ireland. Additional support is avallable from local road safety officers, but the exact nature of that relationship is not clear (i.e., the extent of the formal or informal basis for supportl. Some parents form local clubs to provide direct training to children, while others instruct their children on an individual basis within the home.

- Parents pay a small fee for enrolling a child in the club and the Tufty Club materials (which include a badge for the child and the Tufty Book of road safety stories). 
- RoSPA encourages meeting clubs to meet once a month at a regular meeting place. Parents are encouraged to form clubs within existing organizations such as local welfare clinics, schools, playgrounds, Sunday schools and women's organizations. All meeting clubs should regist, er with local RoSPA headquarters. There is no charge for affiliation. Affiliated clubs receive free literature and newsletters every school term and can purchase Tufty materials in bulk. The manner in which each club operates is left to the discretion of the parents. Some groups have separate meetings for parents and children occurring simultaneously. For example, a road safety of ficer may talk with parents while the children are having instruction in another room. Other groups mix parents and children together. RoSPA suggests that children should be divided into groups not exceeding ten children per adult leader for indoor instruction and five (or less) children to one adult for outdoor road training. Children's training sessions should not exceed fifteen minutes. RoSPA gives each club a list of basic equipment needed for a Tufty club. Such equipment includes the Tufty books, a Leaders Manual, attendance cards, songs, puzzles, badges, and pictorial aids, etc. Clubs can also obtain these materials and others, such as sildes and films, designed by RoSPA.

6. Materials reviewed: None.

7. Concepts taught:

- Children should never cross the road without being accompanied by an adult.

- Safe places to cross the road

- Use of the curb

- How to cross the street (simplified form of the Green Cross Code)

- $\quad$ thers.

8. Teaching methods used:

- Tufty stories (which usually feature animals learning about road safety principles)

- On-street traffic training

- Songs

- Coloring materials

- Flannelboard

- Jigsaw puzzles 
- Games

- Slides and films

- Leaflets for parents

- Puppets

- Large and small cardboard display figures

- Posters.

N.B. The above information was obtained from the "Tufty Club Information Notes" by RoSPA and the "Tufty Club Teaching Material form and Price List." 


\section{UNITED KINGOOM}

Name of Program: Child Safety Campaigns

1. Sponsor: Department of the Environment.

2. Kinds of child safety campaigns:

- 1971 Child Safety Campaign:

-- Introduction of Green Cross Code.

1975 Safety Campaign:

-- "Lesson for Life Leaflet" was sent to parents through the schools to persuade more parents to take greater involvement in the road safety of their children.

-- Two television spots, one aimed at parents of children under five and one aimed at parents of children between five and nine, directed parents' attention to the "Lesson for Life Leaflet," suggested a few ways to teach children traffic safety, and emphasized the parent's responsibility for teaching their children road safety.

1976 Safety Campaign:

-- Three Green Cross Man television commercials taught children road safety rules. (The Green Cross Man is a super-hero who promotes safety.)

- Cartoon strips of the Green Cross Man appeared in children's comics.

-- Posters of the Green Cross Man were displayed on 3,700 outdoor sites for a four-week period during the campaign.

-- Action posters on buses warned motorists to watch out for children.

-- Wall charts of the Green Cross Man and "Lesson for Life" teaching leaflets were given to schools.

-- Two television commercials informed parents of the dangers possibly confronting their children and suggested ways these dangers could be avoided. 
- 1978 Safety Campaign:

-- The Green Cross Man, a 6'6" British ex-heavyweight champion dressed in his Green Cross costume, toured British schools in his space age car during the fall and taught children how to follow the Green Cross Code. He also told them to use reflective material on their outdoor wearing apparel. He conducted a road safety demonstration near the school and taught the children the Green Cross Code song.

-- A television campaign featuring the Green Cross Man taught road safety precautions to children between the ages of five and twe ive.

-- There was also a road safety television campatgn aimed at mothers of five to nine-year-old children.

3. Possible effect of the campaigns:

- Since the introduction of the Green Cross Code in 1971, child pedestrian casualties in the under fourteen age group fell each year except 1977, during which there was a slight increase. No formal evaluations of the program, however, have been conducted.

N.B. Source of this information: Department of the Environment Press Notices $9 / 1 / 76 ; 10 / 16 / 75 ; 10 / 16 / 75 ; 9 / 19 / 78$. 


\section{APPENDIX A: REFERENCES}

Ainsworth, M.D.S. The development of infant-mother attachment. In B.M. Caldwell \& H. N. Ricciuti (Eds.), Review of child development research, (Vol. 3). Chicago: University of Chicago Press, 1973.

American Automobile Association. Preschool children in traffic.

Falls Church, Va., 1976.

American Automobile Association. The young pedestrian.

Washington, D.C., 1965.

Anderson, D.R., \& Leven, S.R. Young children's attention to "Sesame Street." Child Development, 1976, 47, 806-811.

Asch, S.E., \& Nerlove, H. The development of double function terms in children: An exploratory investigation. In B. Kaplan \& S. Hapner, (Eds.), Perspectives in psychological theory. New York: International Universities, 1960.

Avery, G.C. The capacity of young children to cope with traffic systems: a review. New South Wales: Traffic Research Unit, Department of Motor Vehicles, June 1974.

Avery, G.C., \& Day, R.M. Relationship between the horizontal-vertical illusions for velocity and extent. Journal of Experimental Psychology, $1971,89,22-31$.

Backett, E.M., \& Johnston, A.M. Social patterns of road accidents to children: Some characteristics of vulnerable families. British Medical Journal, February 14, 1959, pp. 409-413.

Baldwin, A.L. Behavior and development in childhood. New York: The Oryden Press, 1955.

Bandura, A. Influence of model's reinforcement contingencies on the acquisition of imitative responses. Journal of Personality and Social Psychology, 1965, 1, 589-595.

Bandura, A. The role of modeling processes in personality development. In W. H. Hartup \& N. L. Smothergill (Eds.). The young child. Washington, D.C.: National Association for the Education of Young Children, 1967.

Bandura, A., Ross, D., \& Ross, S. A. A comparative test of the status, envy, social power and secondary reinforcement theories of identificatory learning. Journal of Abnormal and Social Psychology, 1963, 67, 527-534. 
Bandura, A., \& Walters, R. H. Social learning and personaltty development. New York: Ho1t, Rinehart and Winston, 1963.

Barfenstam, R. Discussion on children and young people in road traffic. Proceedings of the Second Congress of the International Association for Accident and Traffic Medicine, Stockolm, Sweden, August 9-12, 1966.

Barlow, H.B. Single units and sensation: A neuron doctrine for perceptual psychology? Perception, 1972, I, 371-394.

Barlow, H.B., Hill, R.M., \& Levick, H.R. Retínal ganglion cells responding selectively to direction and speed of image motion in the rabbit. Journal of Physiology, 1964, 173, 377-407.

Bartholomew, W.M. Pedestrian accidents in service areas of selected city recreation facilities. Traffic Safety Research Review, 1967, 4, $177-120$.

Baumrind, D. Socialization and instrumental competence in young children. In H. H. Hartup \& N. L. Smothergill (Eds.), The young child. Washington, D.C.: National Association for the Education of Young Children, 1967.

Belmont, J.M., \&utterfield, E.C. The relations of short-term memory to development and intelligence. In L.P. Lipsitt, \& H.H. Reese, (Eds.), Advances in child development and behavior, (Vo1. 4). New York:

Academic Press, 1969.

Berg, W.K., \& Berg, K.M. Psychophysiological development in infancy: State, sensory function, and attention. In J.D. Osof sky, (Ed.). Handbook of Infant Development. New York: Wiley Sons, 1979.

Berger, H.G. Urban pedestrian accident countermeasures experimental evaluation. Volume I.: Behavioral evaluation studies. Falls Church, Va.: Bío Technology, Inc., February 1975.

Berry, J.H. Muller-Lyer susceptibility: Culture, ecology or race? International Journal of Psychology, 1971, 6, 193-197.

Biehler, R. F. Child development: An introduction. Boston: Houghton Miffilin, 1976.

Blackman, A. The role of city planning and child pedestrian safety. Berke ley: University of California, Institute of Urban and Regional Development, 1967.

Blake, J. Developmental change in visual information processing under backward masking. Journal of Experimental Child Psychology, 1974, 17, 133-146. 
Blakemore, C., \& Cooper, G.F. Development of the brain depends on the visual environment. Nature, 1970, 228, 477-478.

Blomberg, R.D., \& Preusser, D.F. Safety messages for public education programs. Final report. Darien, Conn.: Dunlap and Associates, March 1975.

Bobroff, A. The stages of maturation in socialized thinking and in the ego development of two groups of children. Child Development, 1960, 31, 321-338.

Bower, T.G.R. The visual world of infants. Scientific American, 1966, $215,80-92$.

Bower, T.G.R. Development in infancy. San Francisco: W. H. Freeman, 1974.

Bower, T.G.R. The perceptual world of the child. Cambridge, Mass.: Harvard University Press, 1977.

Bower, T.G.R., Broughton, J.M., \& Moore, M.K. Infant responses to approaching objects: An indicator of response to distal variables. Perception and Psychophysics, 1970, 9, 193-196.

Bower, T.G.R., \& Hishart, J.G. The development of auditory-manual co-ordination. In T.G.R. Bower, Development in infancy. San Francisco: W.H. Freeman, 1974.

Bowlby, J. Attachment and 105s. Attachment, (Vol. 1). New York: Basic Books, 1969.

Boyle, D., \& Gilhooly, M.L. Conceptual problems in teaching children road safety. Psychological Dept., University of Aberdeen, 1972. (as cited in Vinje (1978)).

Brake, N., \& Reiss, M.L. Young pedestrian traffic safety education. Paper presented at the 58 th Annual Meeting of the Transportation Research Board, January 1979.

Bransford, J.D., \& Franks, J.J. The abstraction of linguistic ideas. Cognitive Psychology, 1971, 2, 331-350.

Brazelton, T.B. Toddlers and parents. New York: Delta, 1974.

Brian, C.R., Goodenough, F.L. The relative potency of color and form perception at different ages. Journal of Experimental Psychology, 1929, 12, 197-213.

Breckenridge, M. E., \& Murphy, M. N. Growth and development of the young child. Philadelphia: W. B. Saunders, 1969. 
Brown, A.L. The development of memory: Knowing, knowing about knowing. and knowing how to know. In H.W. Reese (Ed.), Advances in child development and behavior, (Vo1. 10). New York: Academic, 1975.

Brown, J.F. The visual perception of velocity. Psychologie Forschung, 1931, 14, 199-232. (as city in Avery (1974)).

Bruner, J.S. The process of education. Cambridge, Mass.: Harvard University Press, 1962.

Bruner, J.S. Beyond the information given: Studies in the psychology of knowing. New York: W.W. Norton, 1973.

Bruner, J. The importance of play. In R. Lewin (Ed.), Child Alive. London: Maurice, Temple, Smith Ltd., 1974.

Cameron, M.H., Stanton, H.G., \& Milne, P.W. Pedestrian accidents and exposure in Australia. Paper presented at the International Conference on Pedestrian Safety, Haifa, Israel, December 1976.

Campos, J.J., Langer, A., \& Krowitz, A. Cardiac responses on the visual cliff in prelocomotor human infants. Science, 1970, 170, 196-197.

Carison, V.R. Adaptation in the perception of visual velocity. Journal of Experimental Psychology, 1962, 64, 192-197.

Cass, J. The significance of children's play. London: B. T. Batsford, Ltd., 1971 .

Catell, R., \& Lewis, G.D. Children's understanding of words used in road safety 1 terature. (TRRL Supplementary Report 1550C). Crowthorne, England: Transportation and Road Research Laboratory, 1975.

Cermak, L.S., Sagotsky, G., \& Moshier, C. Development of the ability to encode within evaluative dimensions. Journal of Experimental Child Psychology, 1972, 13, 210-219.

Chaloner, L. Feeling and perception in young children. London: Tavistock Publications, 1963.

Chukovsky, K. From two to five. Berkeley, Calif.: University of California, 1963.

Cohen, S., Glass, D.C., \& Singer, J.E. Apartment noise, auditory discrimination and reading ability in children. Journal of Experimental Social Psychology, 1973, 9, 407-422.

Coie, J.E., Costanzo, P.R., \& Farnill, D. Specific transitions in the development of spatial perspective taking ability, Developmental Psychology, 1973, $9,167-177$. 
Colbourne, H.V. Factors affecting the safety of young children as pedestrians. Unpublished thesis, 1972. (as cited in Routledge, et al. (1974)).

Cook, F.M. Ability of children in color discriminations. Child Development, 1931, 2, 303-320.

Coote, M. Why young children dash into the road. Safety Education, Autumn, 1976, pp. 3-6.

Cosgrove, J.M., \& Patterson, C.J. Generalization of training for children's listener skilis. Child Development, 1978, 49, 513-516.

Cosgrove, J.M., \& Patterson, C.J. Plans and the development of listener skills. Developmental Psychology, 1977, 13, 557-564.

Cratty, B.J. Perceptual and motor development in infants and children. Englewood Cliffs, N.J.: Prentice-Ha11, 1979.

CRM Books. Developmental psychology today. Del Mar, California, 1971.

Day, M.C. Developmental trends in visual scanning. In H.W. Reese (Ed.). Advances in child development and behavior, (Vol. 10). New York: Academic Press, 1975.

Day, M.C. Visual search by children: The effect of background variation and the use of visual cues. Journal of Experimental Child Psychology, 1978, 25, 1-16.

DeSilva, H.R. Why we have automobile accidents. New York: J. Wiley and Sons, 1947

Dickson, H.P. An instructional device for teaching verbal skills through structured interactions between children in a communication game. Unpublished doctoral dissertation, Stanford University, 1974.

Eagles, E.L., Wishik, S.M., Doerfier, L.G., Melnick, W., \& Levine, H.S. Hearing sensitivity and related factors in children. St. Louis, Mo.: Laryngoscope, 1963.

Eckerman, C.0., What ley, J. L., \& Kutz, S. L. Growth of social play with peers during the second year of life. Developmental Psychology, $1975,11,1,42-49$.

Eckert, H.M., \& Eichorn, D.H. Developmental variability in reaction time. Child Development, 1977, 40, 452-458.

Eifermann, R.R. Social play in childhood. In R.E. Heron \& B. Sutton-Smith (Eds.), Child's play. New York: Wiley, 1971. 
Eimas, P.D. Multiple cue discrimination in children. Psychological Record, 1969, 19, 420-425.

Ekstrom, G., Gastrin, V., \& Quist, 0. Traffic injuries and accident proneness in childhood. Paper presented at the Second Congress of the International Association for Accident and Traffic Medicine, Stockholm, Sweden, August 9-12, 1966.

Elkind, 0 . Children and adolescents: Interpretive essays on Jean Piaget. New York: Oxford University Press, 1974.

Elkind, D., Koegler, R.R., Go, E. Studies in perceptual development II: Part-whole perception. Child Development, 1964, 35 , $81-90$.

Elliott, R. Physiological activity and performance. Psychological Monographs, 1964, 78, No. 10.

Elliott, R. Simple reaction time: Effects associated with age, preparatory interval, incentive-shift and mode of presentation. Journal of Experimental Child Psychology, 1970, 9, 86-107.

Erke, H. Psychologische Untersuchungen zum Uberquerungsverhalten der Fussganger in Abhangigheit von Farb - und Formmerkmalen von Kraftfahrzeugen. Zeitschrift Fur Verkehrssicherheit, 1975 21, 173. (as cited in Vinje (1978)).

Feldman, S.S. Children's understanding of negation as a logical operation. Genetic Psychology Monographs, 1972, 85, 3-49.

Felsentha1, H. The developing self: The parent role. In R. Yamamoto (Ed.). The child and his image: Self concept in the early years. Boston: Houghton Miffilin Co., 1972.

Finlayson, H.M. Personality changes of children's perception of cues of danger. University of Highfield, Dept. of Psychology, Southampton, 1972. (as cited in vinje (1978)).

Firth, D.E. The road safety aspects of Tufty Club. (TRRL Report LR 604). Crowthorne, England: Transportation and Road Research Laboratory, 1973.

Flave11, J.H. Developmental studies of mediated memory. In H.W. Reese \& L.P. Lipsitt (Eds.), Advances in child development and behavior,

(Vo 1. 5). New York: Academic, 1970.

Flave 11, J.H. Cognitive Development. Englewood Cliffs, N.J.: Prentice-Ha11, 1977. 
Flavell, J.H., \& Wellman, H.M. Metamemory. In R.V. Kail \& J.W. Hagen (Eds.), Perspectives on the development of memory and cognition. Hillsdale, N.J.: Lawrence Erlbaum, 1977.

Foote, N. N. Sociological factors in childhood accidents. Paper presented at the Conference on Research in Accident Prevention: Behavioral Approaches to Accident Research, West Point, New York, 1961.

Foote, R. Helping pedestrians in urban areas. Paper presented at the 12th International Study Week in Traffic Engineering and Safety, Belgrade, Yugoslavia, September 1974.

Fraiberg, S. H. The magic years. New York: Charles Scribner's Sons, 1959.

Fraisse, P., \& Vautrey, P.: La perception de l'espace, de la vitesse, et du temps chez l'enfant de cinq ans, Enfance, 1952, $\underline{5}, 1-20$. (as cited in vinje (1978)).

Fry, C.L. Training children to communicate to listeners. Child Development, 1966, 37, 674-685.

Fry, C.L. Training children to communicate with listeners who have varying 1istener requirements. Journal of Genetic Psychology, 1969, $114,153-166$.

Furth, H.G., \& Wachs, H. Thinking goes to school: Piaget's theory in practice. New York: 0xford University Press, 1974.

Gagne, R.M. The condittons of learning. New York: Holt, Rinehart and Winston, 1970 .

Gese11, A. The first five years of 1ife. New York: Harper Brothers, 1970.

Gibson, E.J. Principles of perceptual learning and development: New York: Appleton-Century-Crofts, 1969.

Gibson, E.J., Berhman, R., \& Purdy, J. The effect of prior training with a scale of distance on absolute and relative judgements of distance over ground. Journal of Experimental Psychology, 1955, 50, 97-105.

Gibson, E.J., \& Walk, R.R. The "visual cliff." Scientific American, $1960,202,2-9$.

Gibson, J.J. The senses considered as perceptual systems. London: Allen and Unwin, 1968.(a)

Gibson, J.J. What gives rise to the perception of motion? Psychological Review, 1968, 75, 335-346.(b) 
Glucksberg, S., Krauss, R.M., \& Higgins, E.T. The development of referential communication skilis. In F.D. Hor owtiz (Ed.), Review of child development research, (Volume 4). Chicago: University of Chicago Press, 1975.

Glucksberg, S., Krauss, R.M., Weisberg, R. Referential communication in nursery school children: Method and some preliminary findings. Journal of Experimental Child Psychology, 1966, 3, 333-342.

Gorges, R. Zum verkehrskundltchen Wissen von 5 bis 7 jahrigen Kindern. Zeitschrift fur Verkehrserzlehung, 1973, 10-15. (as cited in Vinje (1978)).

Grayson, G.B. The Hampshire child pedestrian accident study. (TRRL Report 668). Crowthorne, England: Transportation and Road Research Laboratory, 1975(a).

Grayson, G.B. Observations of pedestrian behaviour at four sites. (TRRL Report 70). Crowthrone, England: Transportation and Road Research Laboratory, $1975(b)$.

Greenwood, M., \& Woods, H.M. The incidence of industrial accidents with special reference to multiple accidents. (Report No. 4). Industrial Research Board, 1919.

Gummerman, K., \& Gray, C.R. Age, iconic storage and visual information processing. Journal of Experimental Child Psychology, 1972, 13, $165-170$.

Gunther, R., \& Limbourg, M. Bedingungen fur des Erleben und Verhalten von kindern im Strassenverkehr, Unfall und Sicherheitsforschung Strassenverkehr. Heft 4 Bundesanstalt fur Strassenwesen, Kolh, 1976. In a more elaborate version unpublished, Universitat Tubingen, 1974. (as cited by Vinje (1978)).

Haddon, W. Oiscussion on children and young people in road traffic. Proceedings of the Second Congress of the International Association for Accident and Traffic Medicine, Stockholm, Sweden, August 9-12, 1966.

Haddon, W., Suchman, E.A., \& Klein, D. Accident research: Methods and approaches. New York: Harper and Row, 1964.

Haith, M.M., Morrison, F.F., Sheingold, K., \& Mindes, P. Short-term memory for visual information in children and adults. Journal of Experimental Psychology, 1970, 9, 459-469.

Hardgrove, C., \& Dawson, R. Parents and children in the hospital: The family's role in pediatrics. Boston: Little, Brown \& Co., 1972. 
Harter, M.R., Suitt, C.D. Visually-evoked critical responses and pattern vision in the infant: A longitudinal study. Psychonomic Science, 1970, 18, 235-237.

Hartwig, H. Das Einwirken des sozialen Umfeldes auf das Vorschulkind im Strassenverkehr - zugleich ein praktischer Beitrag zur Verkehrserziehung. Arztl, Jugendkunde, 1969, 60, 13-23. (as cited in OECD (1978)).

Hartwig, H. Zum Entwicklungsstand der motorischen, sensorischen und intellektuellen Fahigkeiten des Vorschulkindes unter dem Aspekt seiner Teilnahme am Strassenverkehr, Arztliche Jugenkunde, 1968, 59, 345-364. (as cited in Vinje (1978)).

Heinrich, H. Ch., \& Langosh, J. Wirkungen verschiedener Unterrichtsformen bef der Verkehrserziehung im ersten Schuljahr. Zeitschrift fur Verkehrserziehung, 1976, 1, 3-12. (as cited in Vinje (1978)).

Helms, 0. B., \& Turner, J. S. Exploring child behavior. Philadelphia: W. B. Saunders, 1976.

Hetherington, E.M., \& Morris, W.N. The family and primary groups. In W.H. Holtzman (Ed.), Introductory psychology in depth. New York: Harper and Row, 1978.

Hetherington, E.M., \& Parke, R.D. Child psychology: A contemporary viewpoint. New York: McGraw-Hill, 1979.

Hirsch, H.V.B., \& Spinelli, D.N. Visual experience modifies distribution of horizontally and vertically oriented receptive fields in cats. Science, 1970, 168, 869-871.

Hoffman, E.R., Payne, A, \& Prescott, S. Children's estimates of vehicle approach times. Paper presented at Joint ARRB/DOT Pedestrian Conference, Sydney, N.S.W., November, 1978.

Hohmann, M., Banet, B., \& Weikart, D. Young children in action. Ypsilanti, Mich.: The High/Scope Press, 1979.

Hoshi, T. Educating young children for traffic safety: Activities of the children's traffic safety clubs. The Wheel Extended, 1976, 6 , 14-18.

Hoving, K.L., Morin, R.E., \& Konick, O.S. Recognition reaction time and size of the memory set: A developmental study. Psychonomic Science, $1970,21,247-248$.

Howarth, C.I. Unpublished manuscript. Nottingham, England: University of Nottingham, 1971. (as cited by Reiss (1975)). 
Hoy, E.A. Predicting another's visual perspective: A unitary skill? Developmental Psychology, 1974, 10, 462.

Hube 1, D.H., \& Wiesel, T.N. Receptive fields of single neurons in the cat's striate cortex. Journal of Physiology, 1959, 148, 574-591.

Hube 1, O.H., \& Wiesel, T.N. Receptive fields, binocular interaction, and functional architecture in the cat's visual cortex. Journal of Physiology, 1962, 160, 106-154.

Hube1, D.H., \& Hiesel, T.N. Binocular interaction in striate cortex of kittens reared with artificial squint. Journal of Neurophysiology, $1965,28,1041-1059$. (a)

Hubel, D.H., \& Wiesel, T.N. Receptive fields and functional architecture in two nonstriate visual areas (18 and 19) of the cat. Journal of Neurophysiology, 1965, 28, 229-289.(b)

Hube1, D.H., \& Wiese1, T.N. Receptive fields and functional architecture of monkey striate cortex. Journal of Physiology, 1968, 195, 215-243.

Hube1, D.H., Wiese1, T.N. The period of susceptibility to the physiological effects of unilateral eye closure in kittens. Journal of Neurophysiology, 1970, 906, 419-436.

Huelke, D.F., \& Davis, R.A. Pedestrian fatalities report from Highway Safety Research Institute. (No. Bio. 9). Ann Arbor: University of Michigan, 1969.

Hur lock, E. Child development. New York: McGraw-Hill, 1956.

Husband, $P$. The accident-prone child. The Practitioner, 1973, 211, 335-344.

Hymes, J.L., Jr. The child under six. Englewood Cliffs, N.J.: Prentice-Ha 11, 1963.

Illingworth, R.S. The development of the infant and young child, normal and abnormal. New York: Churchill Livingstone, 1975.

Isaacs, S, Socjal development in young children. New York: Schocken Books, 1972.

Ittelson, W.H. Size as a cue to distance: State localization. American Journal of Psychology, 1951, 64, 54-67.

Jacobs, G.P., \& Wilson, D.G. A study of pedestrian risk in crossing busy roads in four towns. (TRRL Report LR106). Crowthorne, England: Transportation and Road Research Laboratory, 1967. 
Jarvis, J.R. The pedestrian accident situation in Australia.

Paper presented at the Joint ARRB/DOT Pedestrian Conference, Sydney, N.S.H., November 1978.

Jersild, A.T. Child psychology. Englewood Cliffs, N.J.: Prentice-Ha11, 1968.

Kagan, J. The concept of identification. Psychological Review, 1958, 65, 296-305.

Kagan, J., \& Kogan, N. Individuality and cognitive performance. In P.H. Mussen (Ed.), Carmichael's manual of child psychology, (Vol. 1). New York: John Hiley \& Sons, 1970.

Kagan, J., \& Lemkin, J. Form, color, and size in children's conceptual behavior. Child Development, 1961, 32, 25-28.

Kenne 1, F. Personal Interview, 1980.

Kidd, A.H., \& Kidd, R.M. The development of auditory perception in chilidren. In A.H. Kidd \& J.L. Rivoire (Eds.), Perceptual development in children. London: University of London Press, 1966.

Kimura, 0 . Speech lateralization in young children as determined by an auditory test. Journal of Comparative Physiology and Psychology, $1963,56,899-902$.

Kimura, D. Left-right differences in the perception of melodies. Quarter ly Journal of Experimental Psychology, 1964, 16, 355-358.

Knoblauch, R.L. Urban pedestrian accident countermeasures experimental evaluation. Volume II - Accident studies. Falls Church, Va.: Bio Technology, Inc., February 1975.

Knoblauch, R.L. Causative factors and countermeasures for rural and suburban pedestrian accidents: Accident data collection and analysis. Falls Church, Va.: Bio Technology, Inc., March 1977.

Kobasigawa, H. Utilization of retrieval cues by children in recall. Child Development, 1974, 45, 127-134.

Kogan, N. Cognitive styles in infancy and early childhood. Hillsdale, N.J.: Lawrence ErTbaum, 1976.

Kohn, M. Class and conformity. Chicago: University of Chicago Press, 1977.

Krauss, R.M., \& Glucksberg, S. The development of communication: Competence as a function of age. Child Development, 1969, 40, 255-266. 
Lafond, D. Personal Interview, 1980.

Lambert, W.W., \& Lambert, E.C. Some indirect effects of reward on children's size estimations. Journal of Abnormal and Social Psychology, 1953, 48, 507-510.

Lambert, W.W., Solomon, R.L., \& Watson, P.D. Reinforcement and extinction as factors in size estimation. Journal of Experimental Psychology, 1949, 39, 637-641.

Levick, W.R. Receptive fields of rabbit retinal ganglion cells. American Journal of Optometry, 1965, 42, 337-343.

Lindensjo, S., Mattson, K., \& Sande 1s, S. The Skandia report I. Stockholm: Skandia, 1971.

Lindstrom, S. Discussion on children and young people in road traffic. Proceedings of the Second Congress of the International Association for Accident and Traffic Medicine, Stockhoim, Sweden, August 9-12, 1966.

Liss, P.H., \& Haith, M.M. The speed of visual processing in children and adults: Effects of backward and forward masking. Perception \& Psychophysics, 1970, $8,396-398$.

Logan, J. The child in Detroit traffic. Report from Detroit Police Department, Traffic Safety Bureau, 1963.

Love11, K., Kellett, V.L., \& Moorhouse, E. The growth of the concept of speed: A comparative study. Journal of Child Psychology and Psychiatry, 1962, 3, 101-110.

Luria, A.R. The nature of human conflicts or emotion, conflict and will. New York: Liveright, 1932. (as cited in Cratty (1979)).

Luria, A.R. The working brain: An introduction to neuropsychology. (B. Haigh, trans.) Penguin Books: Harmondsworth, 1973.

Lykes, N.R. A psychological approach to accidents. New York: Vantage Press, 1954.

Maccoby, E.E. The development of stimulus selection. In J.P. Hill (Ed.), Minnesota symposia on child psychology, (Vol. 3). Minneapolis: University of Minnesota Press, 1969.

Mackworth, N.H., \& Bruner, J.S. How adults and children search and recognize pictures. Human Development, 1970, 13, 149-177. 
Magnusson, G. Traffic accidents among children: A critical survey and bibliography of articles reported in the Index Medicus from 1937 to 1965. Paper presented at the Second Congress of the International Association for Accident and Traffic Medicine, Stockholm, Sweden, August 9-12, 1966.

Maier, N.F.R. Psychology in industry. Boston: Houghton Miffin Co., 1946.

Maier, N.F.R. Psychology in industrial organizations. Boston: Houghton Miffiin, Co., 1973.

Maier, N.R. Reasoning in children. Journal of Comparative Psychology. $1936,21,357-366$.

Manheimer, D.I., \& Mellinger, G.D. Personality characteristics of the child accident repeater. Child Development, 1967, 38, 491-513.

Mardriota, F.J., Mintz, D.E., \& Notterman, I.M. Visual velocity discrimination: Effects of spatial and temporal cues. Science, $1962,138,437-438$.

Marg, E., Freeman, D.N., Peltzman, P., Goldstein, P.J. Visual acuity development in human infants: Evoked potential measurements. Investigative Opthalmology, 1976, 15, 150-153.

Markman, E. Factors affecting the young child's ability to monitor his memory. Unpublished doctoral dissertation, University of Pennsylvania, 1973. (as cited in Hetherington \& Parke (1979)).

Marsh, B.W. Traffic control for pedestrians. Washington, D.C.: American Automobile Association, 1955.

Martin, G.L., \& Heimstra, N.W. The perception of hazard by children. Journal of Safety Research, 1973, $5,238-246$.

McDougall, R. Traffic accidents to children. Canadian Medical Journal, $1960,2,61-65$.

Mckelvey, R.K. Development of a safe road crossing interval training procedure for primary school children. (Human Factors Report HFR-9). Melbourne: Monash University Human Factors Group, 1978.

Merchant, R.L., \& Rebelsky, F. Effects of participation in rule formation on the moral judgement of children. Genetic Psychology Monographs, 1972, 85, 287-304.

Messer, S.B. Reflection-impulsivity: a review. Psychological Bulletin, $1976,83,1026-1052$. 
Michalik, C. Traffic knowledge and traffic understanding of 5 to 6 year old children. Unpublished report. (as cited in OECO (1978)).

Miller, L.K. Eye-movement latency as a function of age 1 stimulus uncertainty, and position in the visual field. Perceptual and Motor Ski11s, 1969, 28, 631-636.

Minnigerode, F.A., \& Carey, R.N. Development of mechanisms underlying spatial perspectives. Child Development, 1974, 45, 496-498.

Mintz, A. The inference of accident liability from the accident record. Journal of Applied Psychology, 1954, 38, 41-46.

Molen, H.H. van der. Observational studies of children's road crossing behavior: A review of the literature. In A.S. Hakkert (Ed.), Proceedings of the international conference on pedestrian safety. Haifa: Michiol-Publ House Technion, 1977.

Molen, H.H. van der. Child pedestrian's exposure, accidents and behavior. Draft paper for the OECD workshop "Child pedestrian safety training and techniques," (Report VK 78-02). Groningen: University of Groningen, Traffic Research Centre, 1978.

Mollstedt, B.0. Discussion on children and young people in road traffic. Proceedings of the International Association for Accident and Traffic Medicine, Stockholm, Sweden, August 9-12, 1966.

Mussen, P. H., \& Parker, A. L. Mother nurturance and girls' incidental imitative learning. Journal of Personality and Social Psychology, $1965,2,94-97$.

Neimark, E.D., \& Slotnick, N.S. Development of the understanding of logical connectives. Journal of Educational Psychology, 1970,61, $451-460$.

Neisser, U. Cognitive psychology. New York: Appleton-Century-Crofts, 1967.

Nelson, K.E. Memory development in children: Evidence from nonschool tasks. Psychonomic Science, 1971, 25, 244-248.

Newman, B.B., \& Newman, P.R. Infancy and childhood. New York: John Wi ley and Sons, 1978.

Newman, M. Visual acuity. In R.A. Moses (Ed.), Adler's physiology of the eye, (6th Ed). St. Louis: C. V. Mosby Co., 1975.

Nigil, A.J., \& Fishbein, H.D. Perception and conception in coordination of perspectives, Developmental Psychology, 1974, 10, 858-866. 
Nordentoft, $L$. Child traffic accidents. Paper presented at Proceedings of the International Meeting on Biomechanics of Trauma in Children, Lyons, France, September 1974.

Nummenmaa, T., Ruuhilehto, K., \& Syvanen, M. Traffic education programs for preschool aged children and children starting school. Helsinki, Finland: Lijkenneturva, 1975.

Ocio, B. La representation du danger chez l'enfant dans la circulation. Organisme National de Securite Routiere, Paris, 1978. (as cited ín Vinje (1978)).

Okamoto, $Y$. Children as observed in traffic accidents. International Association of Traffic and Safety Sciences, 1978, 2, 119-126.

01der, S.J., \& Grayson, G.B. Perception and decision in the pedestrian task. (TRRL Supplementary Report 49UC). Crowthorne, England: Transportation and Road Research Laboratory, 1974.

01son, D.R., \& Baker, N.E. Children's recall of spatial orientation of objects. Journal of Genetic Psychology, 1969, 114, 273-281.

Oppenheimer, L. Comprehensive speed perception: Its development and relation to mental imagery. (Report WR750). University of Groningen: Werkgroep Verkeeskunde, 1975. (as cited in Vinje (1978)).

Organization for Economic Cooperation and Development, Special Research Group on Pedestrian Safety. Chairman's report and report of sub-group II. Road safety education, 1978.

Pantle, A.J., \& Sekuler, R.W. Velocity - sensitive elements in human vision: Initial psychophysical evidence. Vision Research, 1968, 445-450.

Paris, S.G., \& Lindauer, B.K. Constructive processes in children's comprehension and memory. In R.V. Kail \& J.H. Hagen (Eds.), Perspectives on the development of memory and cognition. Hillsdale, N.J.: Lawrence Er Ibaum, 1977.

Parsons, T. Family structure and the socialization of the child. In T. Parsons \& R. Bales (Eds.), Family socialization and interaction process. Glencoe, Free Press, 1965.

Parteu, M.B. Social participation among preschool children. Journal of Abnormal and Social Psychology, 1932, 27, 243-269.

Patterson, C.J., Cosgrove, J.M., Gosgrove, J.M. Children's referential communication: Components of plans for effective listening. Developmental Psychology, 1978, 14, 401-406. 
Pease, K., \& Preston, G.. Road safety education for young children. British Journal of Educational Psychology, 1967, $3,305-313$.

Peterson, C.L., Danner, F.W., \& Flave11, J.H. Developmental changes in children's response to three indications of communicative fallure. Child Development, 1972, 43, 1463-1468.

Petrillo, M., \& Sanger, S. Emotional care of hospitalized children: An environemntal approach. Philadelphia: J.P. Lippincott, 1972.

Phillips, J.L., Jr. The origins of intellect: Piaget's theory. San Francisco: Freeman, 1969.

Piaget, J. The language and thought of the child. (M. Gabin, trans.). New York: Harcourt Brace, 1926.

Piaget, J. The child's conception of physical causality. (M. Gabin, trans.). New York: Basic Books, 1930.

Piaget, J. Play, dreams, and imitation in children. (M. Cook, trans.). New York: W.W. Norton, 1951.

Piaget, J. The origins of intelligence in children. (M. Cook, trans.). New York: International Universities Press, 1952.

Piaget, J. The construction of reality in the child. (M. Cook, trans.). New York: Basic Books, 1954.

Piaget, J. The child's conception of movement and speed. (Holloway \& M.T. MacKenzie, trans.). London: Routledge and Kegan Pau1, 1970.

Piaget, J. The psychology of intelligence. (M. Piercy \& D.E. Berlyne, trans.). Totowa, N.J.: Littlefield, Adams, \& Co., 1972.

Piaget, J. The development of thought: Equilibration of cognitive structures. (A. Rosin, trans.). 0xford: B. Blackwe11, 1978.

Piaget, J., \& Inhelder, B. The child's conception of space. (F. Langdon \& J.L. Lunzer, trans.). London: Routledge and Kegan Pau1, 1956.

Pick, A.D., Christy, M.D., \& Franke1, G.W. A developmental study of visual selective attention. Journal of Experimental Child Psychology, 1972, 14, 165-175.

Pick, A.D., \& Frankel, G.W. A developmental study of strategies of visual selectivity. Child Development, 1974, 45, 1162-1165.

Plank, E. N. Working with children. Chicago: Year Book Medical Publishers, Inc., 1971. 
Pollack, R.H. Some implications of ontogenetic changes in perception. In J. Flave 11 \& 0 . Elkind (Eds.), Studies in cognitive development. New York: Oxford University Press, 1969.

Preston, B. Statistical analysis of child pedestrian accidents at Manchester and Salford. Accident Analysis and Prevention, 1972, 4, 323-332.

Price, R.L. Discussion on children and young people in road traffic. Proceedings of the Second Congress of the International Association for Accident and Traffic Medicine, Stockholm, Sweden, August 9-12, 1966.

Rand, G., \& Wagner, S. Ontogenetic changes in the identification of simple forms in complex contexts. Human Development, 1969, 12, 154-168.

Read, J.H., Bradley, E.J., Morison, J.D., Lewa11, 0., \& Clarke, D.A. The epidemiology and prevention of traffic accidents involving child pedestrians. The Canadian Medical Association Journal, October 1963, 89, 687-701.

Reiss, M.L. School Trip Safety and Urban Play Areas. Final Report. Falls Church, Va.: Bio Technology, Inc., November 1975.

Reiss, M.L. Young pedestrian accidents and how to prevent them. Journal of Traffic Safety Education, July 1978, 25, 17-18; 26.

Reiss, M.L., \& Robertson, H.D. Young pedestrian safety: The school walking trip. Falls Church, Va.: Bio Technology, Inc., December 1975.

Richard, H. Protection on the way to school. Zeftschrift fur Verkehrssicherheit, 1975, 2l(3). (as cited in OECD (1978)).

Richmond, A. An introduction to Piaget. New York: Basic Books, 1971.

Rosinski, R.R. The development of visual perception. Santa Monica, Calif.: Goodyear Publishing Company, 1977.

Ross, S.P., \& Seefeldt, C. Young children in traffic: How can they cope? Young Children, May 1978, 68-73.

Rossi, S., \&ittrock M.C. Developmental shifts in verbal recall between mental ages two and five. Child Development, 1971, 42, 333-338.

Rothengatter, J.A. Traffic training of children: A review of literature on the basis of an instructional model. (Report VK 77-05). Groningen: University of Groningen, Traffic Research Centre, 1977. 
Rothengatter, J.A. The influence of instructional variables on the effectiveness of traffic education. (Report VK 78-01). Groningen: University of Groningen, Traffic Research Centre, 1978.

Rothengatter, T. Traffic education in traffic training gardens, a 1iterature review. (Report VK 76-02). Groningen: University of Groningen, Traffic Research Centre, 1976.

Rout ledge, D.A., Repetto-Wright, R.T., \& Howarth, C.I. The exposure of young children to accident risk as pedestrians. Ergonomics, 1974, 17. 457-480.

Russam, $K$. Road safety of children in the United Kingdom. (TRRL Report 678). Crowthorne, England: Transportation and Road Research Laboratory, 1975.

Russam, $K$. The psychology of children in traffic. (TRRL Supplementary Report 2951. Crowthorne, England: Transportation and Road Research Laboratory, 1977.

Saito, R., Nakamur, E., Hoshi, T., Hibi, A. Children's understanding of traffic terms. Reports of the National Research Institute of Police Science, 1978, 19, 20-29.

Salvatore, $S$. The ability of elementary and secondary children to sense oncoming car velocity. Journal of Safety Research, 1974, 6, 118-125.

Sande 1s, S. Young children's ability to understand traffic education. Paper presented at the Second Congress of the International Association for Accident and Traffic Medicine, Stockholm, Sweden, August 9-12, 1966.

Sandels, S. Young children in traffic. British Journal of Educational Psychology, 1970, 2, 108-113.

Sande 1s, S. Why are children injured in traffic? The Skandia report, Stockho 1m: Skandia, 1974.

Sande 1s, S. Children in traffic. (H. Mabon, trans.). London: Elek Books, Ltd., 1975.

Sargent, K.J., \& Sheppard, D. The development of the Green Cross Code. (TRRL Report LR 605). Crowthorne, England: Transportation and Road Research Laboratory, 1974.

Schioldborg, P. Children, traffic and traffic training: An analysis of the "Children's Traffic Club," Os 10: University of Oslo, Psychology Institute, 1974. 
Schneider, W., \& Shiffrin, R.M. Controlled and automatic human information processing: Detection, search and attention. Psychological Review, 1977, 84, 1-66.

Schreiber, J., \& Lukin, J. Communicating road safety to the young pedestrian. (Report 3/78). New South Wales: Department of Motor Transport, Traffic Accident Research Unit, 1978.

Secord, P. R., \& Backman, C. W. Social psychology. New York: McGraw-Hi11, 1964.

Sega 11, M.H., Campbe11, D.T., \& Herskavits, M.J. The influence of . culture on visual perception. Indianapolis: Bobbs-Merri11, 1966.

Sekuler, R.W., \& Ganz, L. After-effect of seen motion with a stabilized retinal image. Science, 1963, 139, 419-420.

Sekuler, R.W., \& Pantle, A.J. A model for after-effects of seen movement. Vision Research, 1967, 7, 427-439.

Shantz, C.U., Watson, J.S. Assessment of spatial egocentrism through expectancy violation. Psychonomic Science, 1970, 18, 93-94.

Shantz, C.U., \& Watson, J.S. Spatial abilities and spatial egocentrism in the young child. Child Development, 1971, 42, 171-181.

Shantz, C.U., \& Wilson, K.E. Training communication skills in young children. Child Development, 1972, 43, 693-698.

Shapiro, B.J., o'Brien, T.C. Logical thinking in children ages six through thirteen. Child Development, 1970, 41, 823-829.

Shatz, M., \& Gelman, R. The development of communication skills: Modifications in the speech of young children as a function of 1istener. Monographs of the Society for Research in Child Development, 1973, 38 (5, Serial No. 152), 1-37.

Shirley, M., \& Poyntz, T. The influence of separation from the mother on children's emotional responses. Journal of Psychology, 1941, 12, 251-282.

Sieganthaler, B., \& Barr, C.A. Auditory and figure ground perception in normal chilren. In Sveriges Radios Publikoch programforskning mr 124/70, I Rydin \& G. Hanson (Eds.), Stockholm, 1970. (as cited in Sande1s $(1975))$.

Sluckin, W. (Ed.). Fear in animals and man. New York: Van Nostrand Reinhold Co., 1979. 
Smith, M.E. An investigation of the development of the sentence and the extent of vocabulary in young children. University of Iowa Studies in Child Helfare, 1926, 3,5 .

Snow, C.E., \&abinovitch, M.S. Conjuctive and disjunctive thinking in Children. Journal of Experimental Child Psychology, 1969, 1, 1-9.

Snyder, M.B., \& Knoblauch R. Pedestrian safety: The identification of precipitating factors and possible countermeasures. Volumes I and Volume II - Appendices. Silver Spring, Md.: Operations Research, Inc., January 1971.

Sokol, S.; \& Dobson, V. Pattern reversal visually evoked potentials in infants. Investigative ophthalmology, 1976, 15, 58-62.

Stack, H.J. History of driver education in the United States. Washington, D.C.: National Education Association, 1966.

Stevens, S.S., \& Newman, E.B. The localization of actual sources of sound. American Journal of Psychology, 1936, 48, 297-306.

Stone, L.J., \& Church, J. Childhood and adolescence. New York: Random House, 1968.

Surwillo, H.W. Human reaction time and period of the EEG in relation to development. Psychophysiology, 1971, $\underline{8}, 468-82$.

Sutton-Smith, B. A syntax for play and games. In R.E. Herron \& B. Sutton-Smith (Eds.), Child's play. New York: Wiley, 1971.

Taba, H., Elzey, F. Teaching strategies and thought processes. Teachers' College Record, 1964, 65, 524-534.

Tanaka, K. Traffic concept of nursery children in local towns. Friends of Nature, November $1965,13,32-33$.

Thackray, R.M., and Dueker, R.L. Child pedestrian supervision/quidance. Final Report. Valencia, Pa.: Applied Sciences, Inc., 1983.

Thrumon, M. The development of concepts of magnitude. Child Deve lopment, 1935, $\underline{6}, 120-140$.

Tighe, L.S., \& Tighe, T.J. Facilitation of transposition and reversal learning in children by prior perceptual training. Journal of Experimental Child Psychology, 1969, 8, 366-374.

Uruno, F., \& Yoshibe, S. The ability of speed judgments among children, Report from the National Research Institution of Police Science, Tokyo, undated. (as cited in Vinje (1978)). 
U.S. Department of Transportation: National Highway Traffic Safety Administration, Pedestrian accident reduction quide. Washington, D.C., 1981.

Vinje, M.P. Children as pedestrians: Abilities and limitations. Draft paper for the OECD Workshop on Pedestrian Safety Education, May 1978.

Vinje, M.P., Groeneveld, J. Understanding visibility in traffic: An experiment with pre-school children. (VK 78-05). Groningen: University of Groningen, Traffic Research Center, in preparation. (as cited in Vinje (1978)).

Vurpillot, E. The development of scanning strategies and their relation to visual differentiation. Journal of Experimental Child Psychology, $1968,6,632-650$.

Vurpillot, E. The visual world of the child. London: George Allen \& Unwin, 1976 .

Walk, R.D. Perceptual learning. In E.C. Carterette \& M.P. Friedman, (Eds.), Handbook of perception, (Vol. 9). New York: Academic Press, 1978 .

Warwick, R. Anatomy of the eye and orbit. Philadelphia: W.B. Saunders Co., 1976.

Weiss, R. Personal interview, 1980.

Wellman, H.M., \& Lempers, J.O. The naturalistic communicative abilities of two-year-olds. Child Development, 1977, 48, 1052-1057.

White, B.L. The first three years of life. Englewood Cliffs, N.J., Prentice-Ha11: 1975.

White, S.H. Evidence for a hierarchical arrangement of learning processes. In L.P. Lipsitt \& C.C. Spiker (Eds.), Advances in child development and behavior, (Vol. 2). New York: Academic Press, 1965.

White S.H. Age differences in reaction to stimulus variation. In $0 . \mathrm{J}$. Harvey (Ed.), Experience, structure and adaptability. New York: Springer, 1966 .

Whitehurst, C.J. The development of communication: Changes with age and modeling. Child Development, 1976, 47, 473-482.

Wiesel, T.N., \& Hubel, O.H. Single cell responses in striate cortex of kittens deprived of vision in one eye. Journal of Neurophysiology, 1963, 26, 1004-1017.

Wiese 1, T.N., \& Hube1, D.H. Comparison of the effects of unilateral and bilateral eye closure or cortical unit responses in kittens. Journal of Neurophysiology, 1965, 28, 1029-1040. 
Wolfe, A. Design of a national pedestrian/pedacyclist exposure data system: Draft interim report. Prepared for National Highway Traffic Safety Administration, Washington, D.C., 1979.

Wolff, P., \& Levin, J.R. The role of overt activity in children's imagery production. Child Development, 1972, 43, 537-548.

Wohiwill, J.F. Overconstancy in distance perception as a function of the texture of the stimulus field and other variables. Perceptual Motor Ski11s, 1963, 17, 831-846.

Hor ld Health Organization. Accidents in childhood. (Technical Report Series, No. 1181. Geneva, 1957.

Wright, J.C., Viliestra, A.G. The development of selective attention: From perceptual exploration to logical search. In H.W. Reese (Ed.), Advances in child development and behavior, (Vol. 10). New York: Academic Press, 1975.

Yeaton, H.H., \& Bailey, J.S. Teaching pedestrian safety skills to young children: An analysis and one-year followup. Journal of Applied Behavior Analysts, 1978, 11, 315-329.

Yoda, A. Children's ability of attention. In S. Itoh (Ed.), Methods to prevent children from accidents. Yuhikaku, May 1973.

Yoshiyuki, 0 . Children as observed in traffic accidents. International Association of Traffic and Safety Sciences Research, 1979, 2, 119-126.

Zaporozhets, A.V. The development of perception in the preschool child. In P.H. Mussen (Ed.), European research in cognitive development, Monographs of the Society for Research in Child Development, 1965, 30, 82-101.

Ziegler, H.P., \& Liebowitz, H. Apparent visual size as a function of distance for children and adults. American Journal of Psychology, 1957, 70, 106-109.

Zinchenko, V.P., Van Chzhi-tsin, B., Tarakanov, V.V. The formation and development of perceptual activity. Soviet Psychology and Psychiatry, 1963, 2, 3-12.

Zwahlen, H.T. Distance judgment capabilities of children and adults in a pedestrian situation. Paper presented at the International Congress of Automotive Safety, San Francisco, California, July, 1974. 


\section{APPENDIX B: LITERATURE REVIEH METHODOLOGY}

\section{Research Methods Used}

To identify and obtain pedestrian safety educational materia1s, Applied Management Sciences utilized the following procedures:

1. A computer search of computerized data bases available to Applied Management Sciences was conducted.

2. A manual search of the holdings of local libraries (state, federal, and university) was completed.

3. Once consent was given by the Contract Technical Manager, we contacted by telephone the governor's highway safety representatives in every contiguous state. Alaska and Hawail, along with the governor's highway safety representatives in the territories of the United States, were contacted via letter. During the initial contact we described our project and our purpose for calling and made inquiries regarding the existence of pedestrian safety programs for the preschool age group, organizations which developed and sponsored such programs, when such programs were developed, the kinds of safety taught, the age of children in the program, the type of program (school, club, home), kinds of programs materials and whether any evaluation of the programs had been made. We requested that program materials be sent to us. The governor's highway safety representatives were most cooperative and supportive. They provided the information requested, sent program materials and a few evaluation reports, and of ten suggested the names of others to contact in public organizations such as the state departments of education and in private organizations. Thus, the information in this document regarding the status of pedestrian safety programs for preschool children in the United States is, for the most part, the information provided by the offices of the governor's highway safety representatives. We are most grateful for their assistance and the assistance of various state departments of education who supplied copies of program materials.

4. We contacted a number of private organizations that have developed pedestrian safety program materials for young children, organizations such as the American Automobile Association, film Loops, Inc., the Automotive Safety Foundation, National Education Association, National Safety Town Center, National Safety Council, and the Sears Roebuck Foundation. Many of these organizations graciously provided us with complimentary copies of their materials.

5. The Highway Safety Research Institute in Ann Arbor, Michigan, ran a computer search of preschool and elementary pedestrian safety programs for us. We also purchased some copies of program materials from their library. 
6. Our panel of consultants was asked to suggest sources of information. Several consultants also lent us copies of relevant program materials from their personal librarles.

7. Through the embassies of Finland, Norway, Sweden, Denmark, the Netherlands, West Germany, Great Britain, Japan, and Australia, and through our reading of child pedestrian accident and research studies, we learned the names and addresses of various organizations that produce or distribute traffic safety materials in foreign countries. We contacted these organizations by mall and were thrilled with the generosity and graciousness with which they replied and the assistance which they gave. They sent complimentary copies of the colorful materials which children in their children's traffic safety programs receive. In order to review these program materials we obtained the services of six translators. Due to financial imitations we were not able to translate all materlals sent from foreign countries, but we belleve that we gained much from seeing their formats and 1 llustrations.

8. Many experts in the field of traffic safety research and education (whom we contacted pursuant to our search for pedestrian research studies) located relevant pedestrian safety program materials for us. 


\section{Algeria}

Mr. Semmache

Directeur Generale De La Surete Nationale

Ministere de l'Interieur

ALGERIA

\section{Australía}

Mr. Richard G. Cox, Chief Traffic Engineer

Traffic \& Safety Department

National Road and Motorists Association

151 Clarence Street

Sydney, New South Wales 2000

AUSTRALIA

Mr. M. O'Rourke

Senior Education Officer

Public Education Section

Box 1839Q, G.P.0.

Melbourne, 3001

AUSTRALIA

Mr. Gordon C. Lewts

General Secretary

National Safety Council of Australia

Federal Secretariat: 2nd Floor

377 Little Collins Street

Melbourne, Victoria 3000

AUSTRALIA

\section{Austria}

Mr. Manhardt

Kuratorium Fur Verkehrssicherheit

$01 z e$ tgasse 3

1031 Wien

AUSTRIA

Belgium

Mr. Dupont, Directeur

Via Secura

Association Nationale Pour La Prevention

Des Accidents De La Route

Rond-Point Robert Schuman 9-Bie 7

1040 Bruxelles

BELGIUM

*The reader is reminded that these addresses were current as of 1981 
Mr. D. Singerman, Sous-Directeur

Via Secura

Association Nationale Pour La Prevention

Des Accidents De La Route

Rond-Point Robert Schuman 9-Bie 7

1040 Bruxelles

BELGIUM

\section{Brazi1}

Dr. Engo Moacyr Berman

Director De Transito-DNER

Endereco: Av. Pres. Vargas

522-90 Andar 20.071

Rio de Janetro - RJ

BRAZIL

\section{Canada}

Mr. Edward Moore, Secretary

Motor Vehicle Safety Association

c/o Ontario Safety League

409 King Street

West Toronto, Ontario M5VIKI

CANADA

Transport Canada

Transport Canada Building

Place de Ville

Ottawa, Ontario KIAON5

CANADA

Mr. S.C. Wilson, Chief

Countermeasures Deve Topment

Mr. Grant Smith, Road User Program Advisor

Road and Motor Vehicle Traffic Safety

Transport Canada

Ottawa, Ontario KIAON5

CANADA

\section{Chile}

Mr. Gufllermo Imas

Club De Seguridad EN EI Transito de Chile Concha y Toro 29

Casilla 13242

Santiago

CHILE 


\section{Dahomey}

Mr. Even

La Prevention Routiere Beninotse

B.P. 743

Contonou

OAHOMEY

\section{Denmark}

Mr. Lars Christensen

Radet For Storre Faerdselssikkerhed

Valbygardsvej 62

2500 Valby

DENMARK

Ministry of Transportation

Frederiksholms Kanal \#25-27

OK-1220 Copenhagen

DENMARK

\section{England}

Mrs. Maeve Denby

Road Safety Foundation Development Unit

17, Granard Avenue

London SW $156 \mathrm{HH}$

ENGLAND

Mr. Charles S. Downing

Road User Characteristics Division

Safety Department

Transport and Road Research Laboratory

Department of the Environment/

Department of Transportation

01d Wokingham Road

Crowthorne

Berkshire RGII $6 \mathrm{AU}$

ENGLAND

Mr. David Larder

Director of Safety Education

The Royal Society for the Prevention of Accidents

Cannon House

The Priory Queensway

Birmingham B4 6BS

ENGLAND

Mr. Michael G. Macdonald

Road Safety General

Department of Transport Room C 16/13

2 Mar sham Street

London SWTP 3EB

ENGLAND 
National Association for Road Safety Instruction in Schools

16 Woodward Avenue

Hendon

London NW4 4NY

ENGLAND

Shell International Petroleum Company (SIPCO), Limited

She 11 Center

London SE 17 NA

ENGLAND

Egyot

Mr. Sayed Zaky

Assoclation De Prevention Routiere Egyptienne

33, Rue Kasr-en-Ni1

Flat 72

Catro

EGYPT

\section{Finland}

Mr. Pauli Eiranen, Head of General Department

Ms. Katrilina Brusila, Educational Planner

Central Organization for Traffic Safety in Finland

ISO Roobertinkatu 20

00120 He 1 sink 12

FINLAND

Mr. Ka lervo Homan

Director of the Education Department

Lilkenneturva

ISO Roobertinkatu 20

00120

Helsinki 12

FINLAND

Mr. Esko Salovaara

Director of the Information Department

Lilkenneturva

ISO Roober linkatu 20

00120 Helsinki 12

FINLAND

\section{France}

Ministere des Transports

Service de Presse et d'Information

32 Avenue du President Kennedy

75775 Par is Cedex 16

FRANCE 
Gabon

$\mathrm{Mr}$. Eyegue

Direction de la Securite Routiere

B.P. 4036

Libreville

GABON

Germany

Mr. M. E. Kerstiens, Dipl.-Pad

ADAC

Zentrale

Baumgartnerstrasse 53

8000 Munich 70

WEST GERMANY

Mr. Hans J. Klotzke

Jugendwerk der Deutschen Shell

Uberseering 35

2000 Hamburg 60

WEST GERMANY

\section{Greece}

Dr. G. P. Macris

Greek Road Federation

9, el. Venizelou Avenue

3rd Floor 1112 office

113 Athens

GREECE

Hungary

Mr. Andras Benket

Counseil National De Securite Routiere

O.K.B.T.

V. Merleg u.3.PF. $314 / 15$

1903 Budapest

HUNGARY

Israe 1

Mr. D. Bar-Giora

Secretary General

The Israel National Council

for the Prevention of Accidents

P.0. Box 17177

9 Fichman Street

Tel Aviv

ISRAEL 
Mr. H. S. Loewenberg

The Israel National Council

for the Prevention of Accidents

P. 0. Box 17177

9 Fichman Street

Tel Aviv

ISRAEL

\section{Ivory Coast}

Mr. Aka

La Prevention Routiere De Cote D'Ivoire

B.P. 20.963

Avenue Verdier

Abidjan

IVORY COAST

\section{Japan}

Traffic Safety Policy Office

Prime Minister's Secretariat

1-6-1 Nagata-Cho

Chiyoda

Tokyo 100

JAPAN

Dr. Tadamichi Hoshi

Director

Office for Safety Educational Research

Japan Society for the Spread of

Traffic Safety Education

Tokyo

JAPAN

Mr. Hitoshi Kikumoto

Mr. Takaharu Yanat

Naikai Foridajin Kanbo Kotsu Anzen

Taisaku Shitsu

161 Nagata-Cho, Chiyoda-ku

Tokyo 100

JAPAN

Mr. Sadao Ichikawa, Manager

Public Nuisance \& Environment Department Environment Section

Ota Ward office

10-1, Chuo 2-Chome, Ota-ku

Tokyo 143

JAPAN 
Mr. Michihiro Nakai, Chief

Information System Department

International Assoctation of Traffic and Safety Sciences

6-20, 2-Chome, Yaesu, Chuo-Ku

Tokyo 101

JAPAN

Shadanhogin Venkoku Kotsu Anzen Haha-no-kal

Rengokai 2-16-6 Hiraga-cho Chiyoda-ku

Tokyo 101

JAPAN

(In reforance to mothers' traffte safety organizations)

Jordan

Association Jordanienne Pour La Prevention Routiere

B.P. 9480

Amman

JORDAN

Kuwait

Mr. Yacoub Malallah

Gulf Automobile Association

P.0. Box 827

Safat

KUWAIT

Luxembourg

Director

The Billy Club

69 Boulevard de la Petrusse

Luxembourg City

LUXEMBOURG

Nether lands

Royal Dutch She11, Inc.

Care 1 Zan Bylandtlaan 30

The Hague

NETHERLANDS

New Zealand

Mr. J. B. Marshall, Secretary

Road Traffic Safety Research Council

P.0. Box 4140

Wellington

NEW ZEALAND 
Norway

Mr. Leif Agnar Ellevset, Managing Director

Ms. Else Karset, Preschool Advisor

Trygg Trafikk

Boks 2610

St. Hanshaugen

Os lo 1

NORWAY

Institute of Transport Economy

Box 6110

Etterstad

Os 106

NORWAY

\section{Poland}

Mr. Niziolek

Polski Swiazek Motorway

U1. Kazimierzowska 66

02-518 War szawa

POLANO

Portugal

Mr. Da Silva

Prevencao Rodoviaria Portuguesa

Rua Rosa Araujo 122

Lisbon

PORTUGAL

Mr. Jose Migue 1 Trigoso

Deputy General Director

Prevencao Rodoviaria Portuguesa

Rua Rosa Araujo 12

1200 Lisbon

PORTUGAL

\section{Senega 1}

Mr. Sene

La Prevention Routiere Senegalaise

43 Avenue Albert Sarraut

B.P. 3349

Dakar

SENEGAL

\section{Sudan}

Mr. Ziada Satti Hamad

Sudan Road Safety Association

P. 0. Box 1185

Khar toum

SUDAN 
Spain

Mr. J. M. Fernandez Cuevas

Direccion General De Trafico

Ministerto del Interior

C. Josefa Valcarcel 28

Madrid 2

SPAIN

\section{South Afrtca}

Mr. Toerien

The National Road Safety Council

83 Beatrix Street

Pretoria 001

SOUTH AFRICA

\section{Sweden}

Mr. Rune EK

Skandia

Department of Public Relations

S-103 60 Stockholm

SWEDEN

Msd. Anita Ericson, Preschool Advisor

Nationalforeningen for Trafiksakerhetens

Framjande

Vartavagen 73

11538 Stockholm

SWEDEN

National Society on Road Safety

Fack

S-17120 Solna

SWEDEN

Mr. K. Nikolaev

Motormannens Riksforbund

Fack

S-10240 Stockholm

SWEDEN

The Swedish Institute

Hamngatan 27

P.0. Box 7434

S-10391 Stockholm

SWEDEN 
Switzer land

Mr. Walthert

Bureau Suisse De Prevention Des Accidents

Laupenstrasse 9-Case 2273

3001 Bern

SWITZERLAND

\section{Tunisia}

Dr. R. Benaicha

Director

Association Tunisienne de la Prevention Routiere

1. Ter, Avenue de Carthage

Tunis

TUNISIA

\section{Turkey}

Mr. Suat Erler

Turkis Traffic Safety Association

P.K. 64 Mecidiyekoy

Istanbu 1

TURKEY

Yugos lavia

Mr. Dimitrijevic

Jugoslovensk1 Savet Za Bezbednost Saobracaja

Slobodana Penezica 35/iv

11000 Beograd

YUGOSLAVIA

\section{Zaire}

Mr. Lobia Haito

La Prozaire, asbl

B.P. 11.316

Kinshasa-1

ZAIRE 